UNIVERSIDAD POLITECNIGA SALESIANA ECUADOR
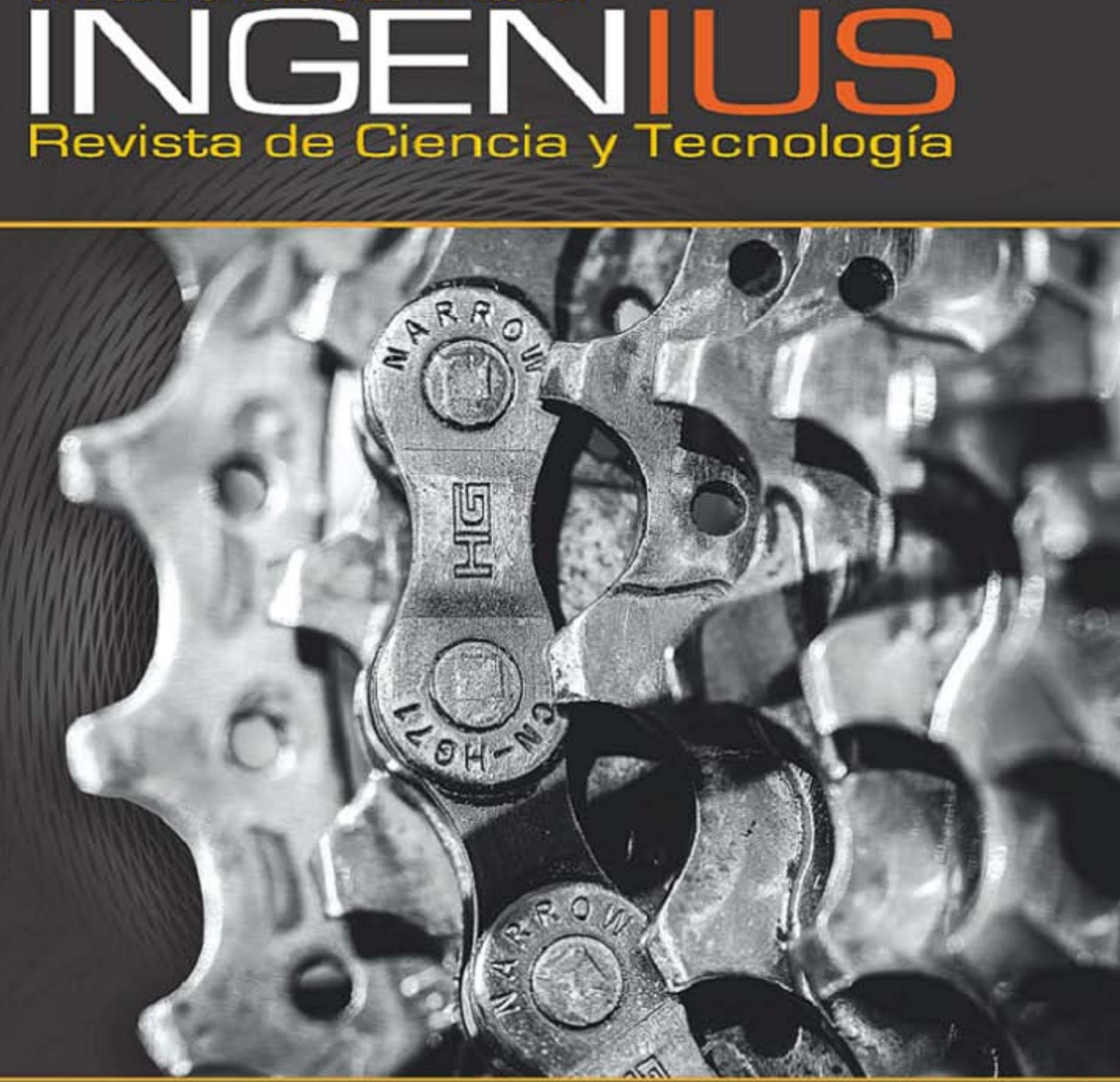

- Caracterización de un generador de flujo axial para aplicaciones en energía eólica Pag, 19

- Factores para seleccionar tuberias de conducción de gas licuado de petróleo en el Ecuador Pag, 51
- Comparación entre redes neuronales artificiales y regresión múltiple para la predicción de la rugosidad superficial / en el torneado en seco. Pag. 79 


\section{INGENIUS}

Ingenius • Número $19 \bullet$ enero/junio 2018. Revista semestral de Ciencia y Tecnología de la Universidad Politécnica Salesiana del Ecuador. Publicación dedicada a estudios relacionados con las Ciencias de la Ingeniería Mecánica, Ingeniería Eléctrica, Ingeniería Eléctronica, Ingeniería Mecatrónica, Ingeniería de Sistemas e Ingeniería Industrial.

\section{Consejo de Editores}

Rafael Antonio Balart Gimeno, PhD, Universidad Politécnica de Valencia, España - Editor Jefe.

\section{Consejo Científico}

Juan López Martínez, PhD, Universidad Politécnica de Valencia, España.

Elena Fortunati, PhD, Universidad de Perugia, Italia.

Gustavo Rovelo Ruiz, PhD, Hasselt University, Diepenbeek, Bélgica.

Franklin Gavilanez Alvarez, PhD, American University, Estados Unidos.

Piedad Gañan Rojo, PhD, Universidad Pontificia Bolivariana, Colombia.

José Alex Restrepo, PhD, Universidad Simón Bolívar, Venezuela.

Sergio Lujan Mora, PhD, Universidad de Alicante, España.

Martha Zequera Díaz, PhD, Pontificia Universidad Javeriana, Colombia.

Grover Zurita, PhD, Universidad Privada Boliviana, Bolivia.

Vladimir RoBles, PhD, Universidad Politécnica Salesiana, Ecuador.

Germán Arévalo, PhD, Universidad Politécnica Salesiana, Ecuador.

Wilbert Aguilar, PhD, Universidad de las Fuerzas Armadas, ESPE, Ecuador.

John Morales García, PhD, Universidad Politécnica Salesiana, Ecuador.

JaCk Bravo Torres, PhD, Universidad Politécnica Salesiana, Ecuador.

Walter Orozco, PhD, Universidad Politécnica Salesiana, Ecuador.

Mariela Cerrada, PhD, Universidad Politécnica Salesiana, Ecuador.

Julio CÉsar Viola, PhD, Universidad Politécnica Salesiana, Ecuador.

Sergio Gamboa SÁnchez, PhD, Universidad Nacional Autónoma de México, México.

Roger Abdón Bustamante Plaza, PhD, Universidad de Chile, Chile.

Christian Blum, PhD, Consejo Superior de Investigaciones Científicas, España.

Silvia Noemi Schiaffino, PhD, Universidad Nacional del Centro de la Provincia de Buenos Aires, Argentina.

Analía Adriana Amandi, PhD, Universidad Nacional del Centro de la Provincia de Buenos Aires, Argentina.
John Ignacio Calle Sigüencia, MSc, Marlon Xavier Quinde Abril, MSc, Universidad Politécnica Salesiana, Universidad Politécnica Salesiana, Ecuador - Editor Responsable.
Rubén de Jesús Medina Molina, PhD, Universidad de Los Andes, Venezuela.

Johnny Josué Bullón Torrealba, PhD, Universidad de Los Andes, Venezuela.

Rodrigo Palma Hillerns, PhD, Universidad de Chile, Chile.

Gerardo Espinoza Pérez, PhD, Universidad Nacional Autónoma de México, México. Alexandre Mendes Abrão, PhD, Universidad Federal de Minas Gerais, Brasil.

Kamla Abdel Radi Ismail, PhD, Universidad Estatal de Campinas Unicamp, Brasil.

Arnaldo Da Silva, PhD, Universidad Estatal de Campinas Unicamp, Brasil.

Álvaro Rocha, PhD, Universidad de Coimbra, Portugal.

José Antenor Pomilio, PhD, Universidad Estatal de Campinas Unicamp, Brasil.

Luis Paulo Reis, PhD, Universidad de Minho, Portugal.

Luís Fernandes, PhD, Escuela Superior Náutica Infante d. Henrique, Portugal.

Aníbal Traça de Almeida, PhD, Universidad de Coimbra, Portugal.

Jorge SÁ Silva, PhD, Universidad de Coimbra, Portugal.

Pedro Manuel Soares Moura, PhD, Universidad de Coimbra, Portugal.

Sérgio Manuel Rodrigues Lopes, PhD, Universidad de Coimbra, Portugal.

Ricardo Madeira Soares Branco, PhD, Universidad de Coimbra, Portugal.

Carlos Alexandre Bento Capela, PhD, Universidad de Coimbra, Portugal.

Filipe Araujo, PhD, Universidad de Coimbra, Portugal.

Luis Manuel Guerra Silva Rosa, PhD, Universidad de Lisboa, Portugal.

Hélder de Jesus Fernandes, Puga, PhD, Universidad de Minho, Portugal.

Filipe Samuel, Pereira da Silva, PhD, Universidad de Minho, Portugal.

César Sequeira, PhD, Universidad de Lisboa, Portugal.

José Teixeira Estêvão Ferreira, PhD,
Universidad de Coimbra, Portugal.

Nuno Laranjeiro, PhD, Universidad de Coimbra, Portugal.

Luís Amaral, PhD, Universidad de Lisboa, Portugal.

Jorge Henriques, PhD, Universidad de Coimbra, Portugal.

William Ipanaque, PhD, Universidad de Piura, Perú.

Lorenzo Leija Salas, PhD, Centro de Investigación y Estudios Avanzados del Instituto Politécnico Nacional, México.

Valeri Kontorovich Mazover, PhD, Centro de Investigación y de Estudios Avanzados del Instituto Politécnico Nacional, México.

Alejandro Ávila García, PhD, Centro de Investigación y de Estudios Avanzados del Instituto Politécnico Nacional, México.

Paolo Bellavista, PhD, Universidad de Bologna, Italia.

Carlos Rubio, PhD, Centro de Ingeniería y Desarrollo Industrial, México.

Fernando Hernández Sánchez, PhD, Centro de Investigación Científica de $\mathrm{Yu}$ catán, México.

Emilio Muñoz Sandoval, PhD, Instituto Potosino de Investigación Científica y Tecnológica, México.

Yasuhiro Matsumoto Kuwabara, PhD, Centro de Investigación y de Estudios Avanzados del Instituto Politécnico Nacional, México.

David Zumoffen, PhD, Centro Internacional Franco Argentino de Ciencias de la Información y de Sistemas, Argentina.

Vicente Rodríguez GonzÁlez, PhD, Instituto Potosino de Investigación Científica y Tecnológica, México.

Alejandro Rodríguez Ángeles, PhD, Centro de Investigación y de Estudios Avanzados del Instituto Politécnico Nacional, México.

Alistair Borthwick, PhD, Universidad de Edimburgo, Reino Unido.

Copyright. Ingenius 2018, Universidad Politécnica Salesiana. Se permite la reproducción total o parcial de esta revista citando la fuente. 
Federico Dominguez, PhD, Escuela Superior Politécnica del Litoral, Ecuador.

Enrique Carrera, PhD, Universidad de las Fuerzas Armadas, ESPE, Ecuador.

Andrés Tello, MSc, Universidad de Cuenca, Ecuador.

Cristian García Bauza, PhD, Universidad Nacional del Centro de la Provincia de Buenos Aires, Argentina.

Osvaldo AÑó, PhD, Universidad Nacional de San Juan, Argentina.

Thalía San Antonio, PhD, Universidad Técnica de Ambato, Ecuador.

Victor Saquicela, PhD, Universidad de Cuenca, Ecuador.

Gonzalo Olmedo, PhD, Universidad de las Fuerzas Armadas, ESPE, Ecuador.

Román Lara, PhD, Universidad de las Fuerzas Armadas, ESPE, Ecuador.

Guillermo Soriano, PhD, Escuela Superior Politécnica del Litoral, Ecuador.

María Fernanda Granda, PhD, Universidad de Cuenca, Ecuador.

Ricardo Cayssials, PhD, Universidad Tecnológica Nacional, Argentina.

Leonardo Solaque Guzman, PhD, Universidad Militar Nueva Granada, Colombia.

José Di PAOLO, PhD, Universidad Nacional de Entre Ríos, Argentina.

Astrid Rubiano Fonseca, PhD, Universidad Militar Nueva Granada, Colombia.

Robinson Jiménez, PhD, Universidad Militar Nueva Granada, Colombia.

Alfonso Zozaya, PhD, Universidad de Carabobo, Venezuela.

Mauricio Mauledoux, PhD, Universidad Militar Nueva Granada, Colombia.

Luis Medina, PhD, Universidad Simón Bolivar, Venezuela.

Ernesto Cuadros-Vargas, PhD, Universidad Católica San Pablo, Perú.

Samuel Sepúlveda Cuevas, PhD, Universidad de la Frontera, Chile.

Carlos Cares, PhD, Universidad de la Frontera, Chile.

RAfael Sotelo, PhD, Universidad de Montevideo, Uruguay.

Omar Lopez, PhD, Universidad de Los Andes, Colombia.

Job Flores-Godoy, PhD, Universidad Católica del Uruguay, Uruguay.

Luis Mario Mateus, PhD, Universidad de los Andes, Colombia.

Amadeo Argüelles Cruz, PhD, Instituto Politécnico Nacional, México.

Santiago Bentancourt Parra, PhD, Universidad Pontificia Bolivariana, Colombia.

Germán Zapata, PhD, Universidad Nacio- nal de Colombia, Colombia.

Pedro García, PhD, Universidad Autónoma de Barcelona, España.

Arturo Conde Enríquez, PhD, Universidad Autónoma de Nuevo León, México.

Alberto Cavazos González, PhD, Universidad Autónoma de Nuevo León, México.

Ernesto Vázquez Martínez, PhD, Universidad Autónoma de Nuevo León, México.

Miguel Díaz Rodriguez, PhD, Universidad de Los Andes, Venezuela.

Efraín Alcorta García, PhD, Universidad Autónoma de Nuevo León, México.

Luis Chirinos Garcia, PhD, Pontificia Universidad Católica de Perú, Perú.

Oscar Avilés, PhD, Universidad Militar Nueva Granada, Colombia.

Dora Martínez Delgado, PhD, Universidad Autónoma de Nuevo León, México.

David Ojeda, PhD, Universidad Técnica del Norte, Ecuador.

Irene Beatríz Steinmann, PhD, Universidad Tecnológica Nacional, Argentina.

Mario Serrano, Universidad Nacional de San Juan, Argentina.

Cornelio Posadas Castillo, PhD, Universidad Autónoma Nuevo León, México.

Mario Alberto Rios Mesias, PhD, Universidad de Los Andes, Colombia.

Yudith Cardinale Villarreal, PhD, Universidad Simón Bolivar, Venezuela.

Eduardo Matallanas, PhD, Universidad Politécnica de Madrid, España.

Jose Eduardo Ochoa Luna, PhD, Universidad Católica San Pablo, Perú.

Dante Angel Elias Giordano, PhD, Pontificia Universidad Católica de Perú, Perú.

Manuel Pelaez Samaniego, PhD, Universidad de Cuenca, Ecuador.

Juan Espinoza Abad, PhD, Universidad de Cuenca, Ecuador.

Pietro Codara, PhD, Universidad de Milan, Italia.

Alberto Soria, PhD, Centro de Investigación y de Estudios Avanzados del Instituto Politécnico Nacional, México.

José M. Aller, PhD, Universidad Politécnica Salesiana, Ecuador.

Ferney Amaya F., PhD, Universidad Pontificia Bolivariana, Medellin, Colombia.

Santiago Arango Aramburo, PhD, Universidad Nacional de Colombia, Colombia.

Diego Arcos-Avilés, PhD, Universidad de las Fuerzas Armadas, ESPE, Ecuador.

Pablo Arevalo, PhD, Universidad Politécnica Salesiana, Ecuador.

Roberto Beltran, MSc, Universidad de las Fuerzas Armadas, ESPE, Ecuador.
Leonardo Betancur, PhD, Universidad Pontificia Bolivariana, Medellin, Colombia.

Roberto Gamboa, PhD, Universidad de Lisboa, Portugal.

Paulo Lopes dos Santos, PhD, Universidad do Porto, Portugal.

Pedro André Dias Prates, PhD, Universidad de Coimbra, Portugal.

José Manuel Torres Farinha, PhD, Universidad de Coimbra, Portugal.

Celso de Almeida, PhD, Universidad Estatal de Campinas Unicamp, Brasil.

Ramon Molina Valle, PhD, Universidad Federal de Minas Gerais, Brasil.

Cristina Nader Vasconcelos, PhD, Universidad Federal Fluminense, Brasil.

João M. Ferreira Calado, PhD, Universidad de Lisboa, Portugal.

Guilherme Luz Tortorella, PhD, Universidad Federal de Santa Catarina, Brasil.

Mauro E. Benedet, PhD, Universidad Federal de Santa Catarina, Brasil.

Artemis Marti Ceschin, PhD, Universidade de Brasilia, Brasil.

Gilmar Barreto, PhD, Universidad Estatal de Campinas Unicamp, Brasil.

Ricardo Emilio F. Quevedo Nogueira, PhD, Universidad Federal de Ceará, Brasil.

Weslley Luiz da Silva Assis, PhD, Universidad Federal Fluminense, Brasil.

Ana P. Martinazzo, PhD, Universidad Federal Fluminense, Brasil.

Jorge Bernardino, PhD, Universidad de Coimbra, Portugal.

Luis Geraldo Pedroso Meloni, PhD, Universidad Estatal de Campinas Unicamp, Brasil.

Facundo Almeraya Calderón, PhD, Universidad Autónoma de Nuevo León, México.

Freddy Villao Quezada, PhD, Escuela Superior Politécnica del Litoral, Ecuador.

Jose Manrique Silupu, MSc, Universidad de Piura, Perú.

Germán Ariel Salazar, PhD, Instituto de Investigaciones en Energía no Convencional, Argentina.

José Mahomar Jananías, PhD, Universidad del BIOBIO, Chile.

Arnaldo Jélvez CaAmaño, PhD, Universidad del BIOBIO, Chile.

Jorge Andrés Uribe, MSc, Centro de Ingeniería y Desarrollo Industrial, México.

Ricardo Beltran, PhD, Centro de Investigación en Materiales Avanzados, México.

Adi Corrales, MSc, Centro de Ingeniería y Desarrollo Industrial, México.

Jorge Uribe Calderón, PhD, Centro de Investigación Científica de Yucatán, México 
José Trinidad Holguín Momaca, MSc, Centro de Investigación en Materiales Avanzados, México.

Juan Manuel Alvarado Orozco, PhD, Centro de Ingeniería y Desarrollo Industrial, México.

Arnaldo Jélvez Caamaño, PhD, Universidad del BIOBIO, Chile.

Javier Murillo, PhD, Centro Internacional Franco Argentino de Ciencias de la Información y de Sistemas, Argentina.

Lucas Daniel Terissi, PhD, Universidad Nacional de Rosario, Argentina.

Rene Vinicio Sanchez Loja, MSc, Universidad Politécnica Salesiana, Ecuador.

Freddy Leonardo Bueno Palomeque, MSc, Universidad Politécnica Salesiana, Ecuador.

Diego Cabrera Mendieta, MSc, Universidad Politécnica Salesiana, Ecuador.

Edwuin Jesus Carrasquero, PhD, Universidad Técnica de Machala, Ecuador.

Carlos Mauricio Carrillo Rosero, MSc, Universidad Técnica de Ambato, Ecuador.

Diego Carrion Galarza, MSc, Universidad Politécnica Salesiana, Ecuador.

Carmen Celi Sanchez, MSc, Universidad Politécnica Salesiana, Ecuador.

Diego Chacon Troya, MSc, Universidad Politécnica Salesiana, Ecuador.

Paul Chasi, MSc, Universidad Politécnica Salesiana, Ecuador.

JuAn Chica, MSc, Universidad Politécnica Salesiana, Ecuador.

Diego Marcelo Cordero Guzmán, MSc, Universidad Católica de Cuenca, Ecuador.

Luis Javier Cruz, PhD, Universidad Pontificia Bolivariana, Medellin, Colombia.

Fabricio Esteban Espinoza Molina, MSc, Universidad Politécnica Salesiana, Ecuador.

Jorge Fajardo Seminario, MSc, Universidad Politécnica Salesiana, Ecuador.

Patricia Fernandez Morales, PhD, Universidad Pontificia Bolivariana, Medellin, Colombia.

Marcelo Flores Vazquez, MSc, Universidad Politécnica Salesiana, Ecuador.

Carlos Flores Vázquez, MSc, Universidad Católica de Cuenca, Ecuador.

Carlos Franco Cardona, PhD, Universidad Nacional de Colombia, Colombia.

Cristian García García, MSc, Universidad Politécnica Salesiana, Ecuador.

Teonila García Zapata, PhD, Universidad Nacional Mayor de San Marcos, Perú.

Luis Garzón MÑoz, PhD, Universidad Politécnica Salesiana, Ecuador.

Natalia Gonzalez Alvarez, MSc, Universidad Politécnica Salesiana, Ecuador.

Ernesto Granado, PhD, Universidad
Simón Bolívar, Venezuela.

Adriana del Pilar Guaman, MSc, Universidad Politécnica Salesiana, Ecuador.

JuAn Inga Ortega, MSc, Universidad Politécnica Salesiana, Ecuador.

Esteban Inga Ortega, PhD, Universidad Politécnica Salesiana, Ecuador.

PaOla Ingavélez, MSc, Universidad Politécnica Salesiana, Ecuador.

Cesar Isaza Roldan, PhD, Universidad Pontificia Bolivariana.

Nelson Jara Cobos, MSc, Universidad Politécnica Salesiana, Ecuador.

Ruben Jerves, MSc, Universidad Politécnica Salesiana, Ecuador.

Victor Ramon Leal, PhD, Investigador de PDVSA, Venezuela

Gabriel Leon, MSc, Universidad Politécnica Salesiana, Ecuador.

Edilberto Llanes, PhD, Universidad Internacional SEK, Ecuador.

Luis López, MSc, Universidad Politécnica Salesiana, Ecuador.

Carlos Mafla Yépez, MSc, Universidad Técnica del Norte, Ecuador.

Hader Martínez, PhD, Universidad Pontificia Bolivariana, Medellin, Colombia

Javier Martínez, PhD, Instituto Nacional de Eficiencia Energética y Energías Renovables, Ecuador.

Alex Mayorga, MSc, Universidad Técnica de Ambato, Ecuador.

Jimmy Molina, MSc, Universidad Técnica de Machala, Ecuador.

Andres Montero, PhD, Universidad de Cuenca, Ecuador.

Vicente Morales, MSc, Universidad Tecnica de Ambato, Ecuador.

Fabián Morales, MSc, Universidad Técnica de Ambato, Ecuador.

Diego Morales, MSc, Ministerio de Electricidad y Energías Renovables del Ecuador.

Yoandrys Morales Tamayo, PhD, Universidad Técnica de Cotopaxi, Cotopaxi

Olena Leonidivna Naidiuk, MSc, Universidad Politécnica Salesiana, Ecuador.

Oscar Naranjo, MSc, Universidad del Azuay, Ecuador.

Paul Narvaez, MSc, Universidad Politécnica Salesiana, Ecuador.

Hernán Navas Olmedo, MSc, Universidad Técnica de Cotopaxi, Ecuador.

Cesar Nieto, PhD, Universidad Pontificia Bolivariana, Medellin, Colombia

Fabio Obando, MSc, Universidad Politécnica Salesiana, Ecuador.

Luis Ortiz Fernandez, MSc, Universidade Federal de Rio Grande del Norte, Brasil

Pablo Parra, MSc, Universidad Politécnica Salesiana, Ecuador.
Paulo Peña Toro, PhD, Ministerio de Productividad, Ecuador.

Patsy Prieto Velez, MSc, Universidad Politécnica Salesiana, Ecuador.

Diego Quinde Falconi, MSc, Universidad Politécnica Salesiana, Ecuador.

Diana Quintana Espinoza, MSc, Universidad Politécnica Salesiana, Ecuador.

William Quitiaquez Sarzosa, MSc, Universidad Politécnica Salesiana, Ecuador.

Flavio Quizhpi Palomeque, MSc, Universidad Politécnica Salesiana, Ecuador.

Washington Ramirez Montalvan, MSc, Universidad Politécnica Salesiana, Ecuador.

Fran Reinoso Avecillas, MSc, Universidad Politécnica Salesiana, Ecuador.

Néstor Rivera Campoverde, MSc, Universidad Politécnica Salesiana, Ecuador.

Jorge Romero Contreras, MSc, Universidad de Carabobo, Venezuela

FABIAN SAEnz Enderica, MSc, Universidad de las Fuerzas Armadas, ESPE, Ecuador.

Luisa Salazar Gil, PhD, Universidad Simón Bolivar, Venezuela

Gustavo Salgado Enríquez, Msc, Universidad Central del Ecuador., Ecuador.

Juan Carlos Santillán Lima, MSc, Universidad Nacional de Chimborazo

Jonnathan Santos Benítez, MSc, Universidad Politécnica Salesiana, Ecuador.

Andrés Sarmiento Cajamarca, MSc, Universidad Federal de Santa Catarina, Brasil

Luis Serpa Andrade, MSc, Universidad Politécnica Salesiana, Ecuador.

Cristian Timbi Sisalima, MSc, Universidad Politécnica Salesiana, Ecuador.

Milton Tipan Simbaña, MSc, Universidad Politécnica Salesiana, Ecuador.

Paul Torres Jara, MSc, Universidad Politécnica Salesiana, Ecuador.

Rodrigo Tufiño CÁrdenas, MSc, Universidad Politécnica Salesiana, Ecuador.

Fernando Urgiles Ortíz, MSc, Universidad Politécnica Salesiana, Ecuador.

Juan Valladolid Quitoisaca, MSc, Universidad Politécnica Salesiana, Ecuador.

Efrén VÁzquez Silva, PhD, Universidad Politécnica Salesiana, Ecuador.

Julio Verdugo, MSc, Universidad Politécnica Salesiana, Ecuador.

Mary Vergara Paredes, PhD, Universidad de los Andes, Merida, Venezuela

Jennifer Yepez Alulema, MSc, Universidad Politécnica Salesiana, Ecuador.

Julio Zambrano Abad, MSc, Universidad Politécnica Salesiana, Ecuador.

Patricia Zapata Molina, MSc, Universidad Politécnica Salesiana, Ecuador. 
Consejo de publicaciones

\author{
Javier Herrán Gómez, sdb, PhD \\ Juan Botasso Boeti, sdb, PhD \\ Juan Pablo Salgado Guerrero, MSc \\ Luis Álvarez Rodas, PhD \\ FAbricio Freire, MSC \\ José Juncosa Blanco, MSc \\ Jaime Padilla Verdugo, MSc \\ Floralba Aguilar Gordón, PhD \\ Sheila Serrano Vicenti, MSc \\ John Calle Sigüencia, MSc \\ René Unda Lara, MSC \\ Betty Rodas Soto, MSc \\ Andrea De Santis, MSc \\ Mónica Ruiz VÁsquez, MSc \\ Editor General
}

Luis Álvarez-Rodas, PhD

\title{
Consejo Técnico
}

Marcia PeÑa, Revisora de Estilo, Centro Gráfico Salesiano - Editorial Don Bosco

Tania Barrezueta, MSc,

Marlon Quinde Abril, MSc, Diagramación y Maquetación

Pablo toasa Reyes

Ángel Torres-Toukoumidis, PhD

\section{Servicio de Publicaciones}

Hernán Hermosa (Coordinación General)

Marco Gutiérrez (Soporte OJS)

Paulina Torres (Edición)

RAysa Andrade (Maquetación)

Martha Vinueza (Maquetación)

\section{Editorial}

Editorial Abya Yala (Quito-Ecuador), Avenida 12 de octubre N422 y Wilson,

Bloque A, UPS Quito, Ecuador.

Casilla 17-12-719 Telfonos: (593-2) 3962800 ext. 2638

Correo electrnico: editorial@abyayala.org

\section{Traductor}

\section{JoAquín CREspo}

Tiraje: 800 ejemplares

Sistema tipográfico usado en la composición de este documento $\mathrm{HAT}_{\mathrm{E}} \mathrm{X}$. 


\section{INGENIUS}

\section{Revista de Ciencia y Tecnología}

Número 19, enero - junio 2018

ISSN impreso 1390-650X / ISSN electrónico 1390-860X

La administración de INGENIUS se realiza a través de los siguientes parámetros:

La revista utiliza el sistema antiplagio académico

Crossref

Similarity Check Powered by
$(U R K \cup N D$
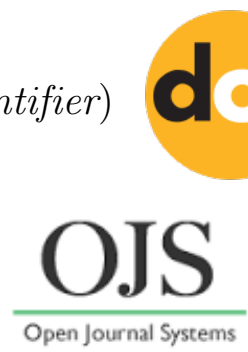

El proceso editorial se gestiona a través del Open Journal System

Es una publicación de acceso abierto (Open Access) con licencia Creative Commons

$$
\text { (c) }
$$

Las políticas copyright y de uso postprint, se encuentran publicadas en el Repositorio de Políticas de Autoarchivo Sherpa/Romeo.

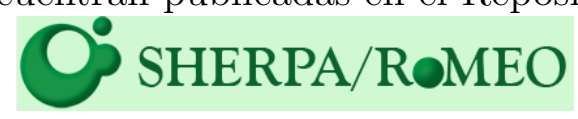

Los artículos de la presente edición pueden consultarse en http://revistas.ups.edu.ec/index.php/ingenius

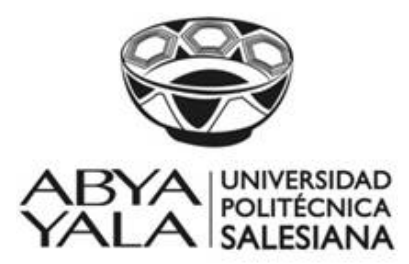

Universidad Politécnica Salesiana del Ecuador 
La Revista Ingenius está indexada en las siguientes Bases de Datos y sistemas de información científica:

BASE DE DATOS SELECTIVAS

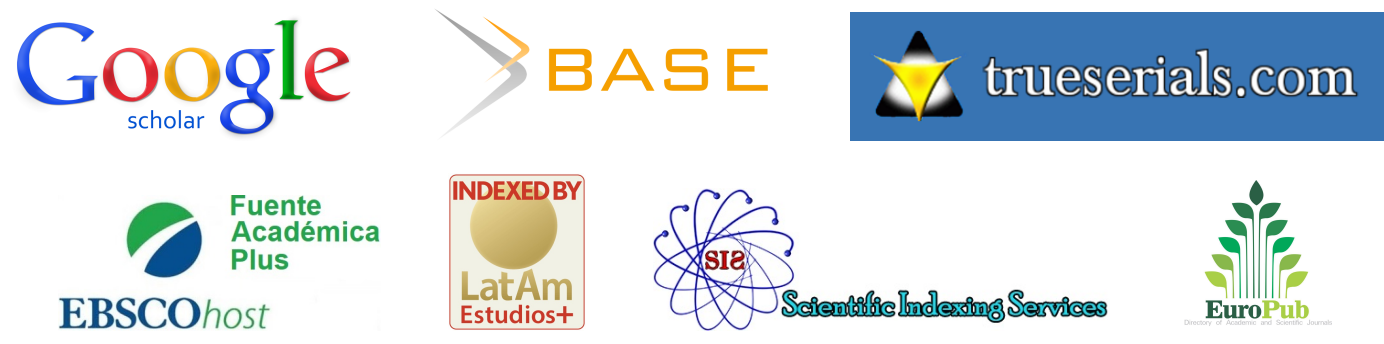

PLATAFORMAS DE EVALUACIÓN DE REVISTAS

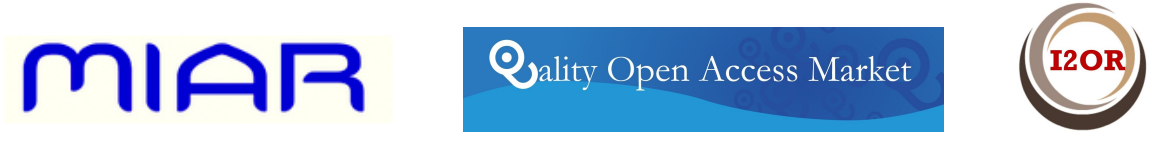

DIRECTORIOS SELECTIVOS
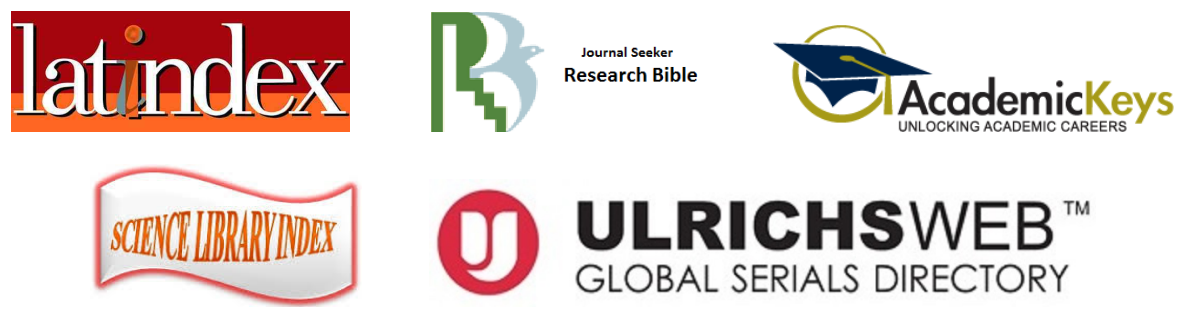

HEMEROTECAS SELECTIVAS

REDIB

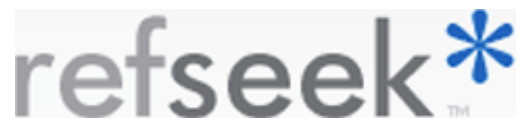

BUSCADORES DE LITERATURA CIENTÍFICA OPEN ACCESS
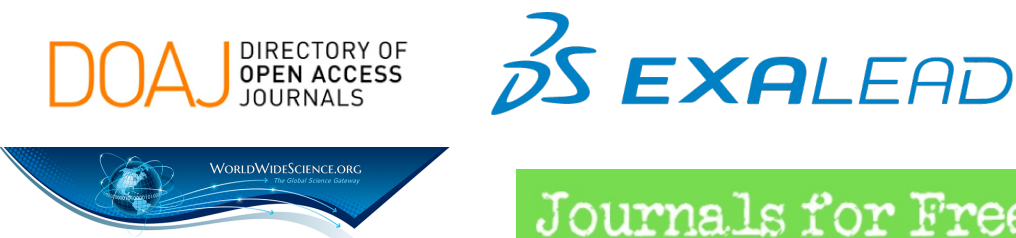

Journals for Firee

OTRAS BASES DE DATOS BIBLIOGRÁFICAS

○ Dialnet 


\section{CATÁLOGO DE BIBLIOTECAS INTERNACIONALES UNIVERSITARIAS}

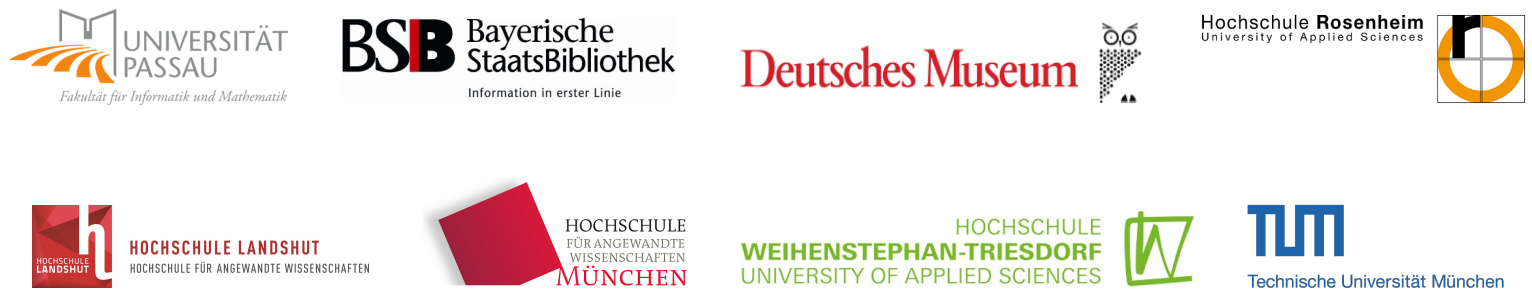

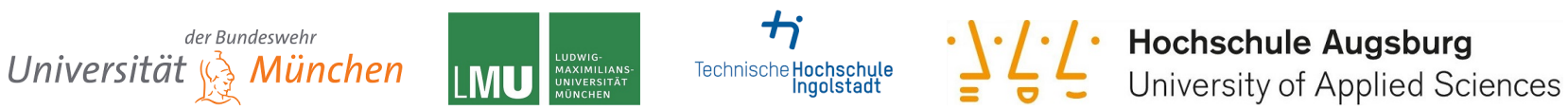

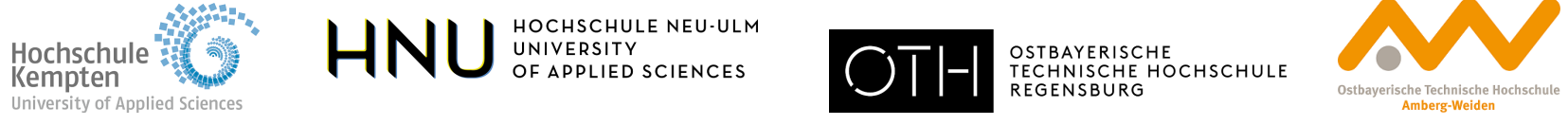

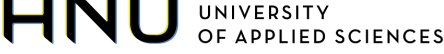

TECHNISCHE HOCHSCHUL

Amberg-Weiden

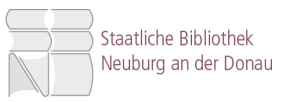

S]

IIIII STAATS-UND

STADTBIBLIOTHEK

IIII AugsBurg

GEORG-SIMON-OHM

HOCHSCHULE NÜRNBERG
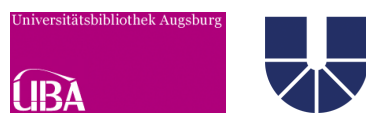

KATHOLISCHE UNIVERSITÄT

EICHSTÄTT-INGOLSTADT

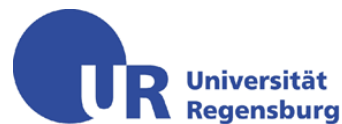

HOCHSCHULE
ANSBACH

COBURG UNIVERSITY
of applied sciences and arts

hochschule
hof
unversity of Apploded Sclonoos

1

HOCHSCHULE

MERSEBURG (FH)

University of Applied Sciences

ThulB

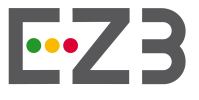

Elektronische

Zeitschriftenbibliothek

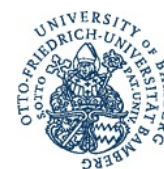

\section{UNIVERSITÄT BAMBERG}

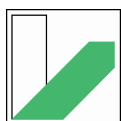

UNIVERSITÄT

BAYREUTH
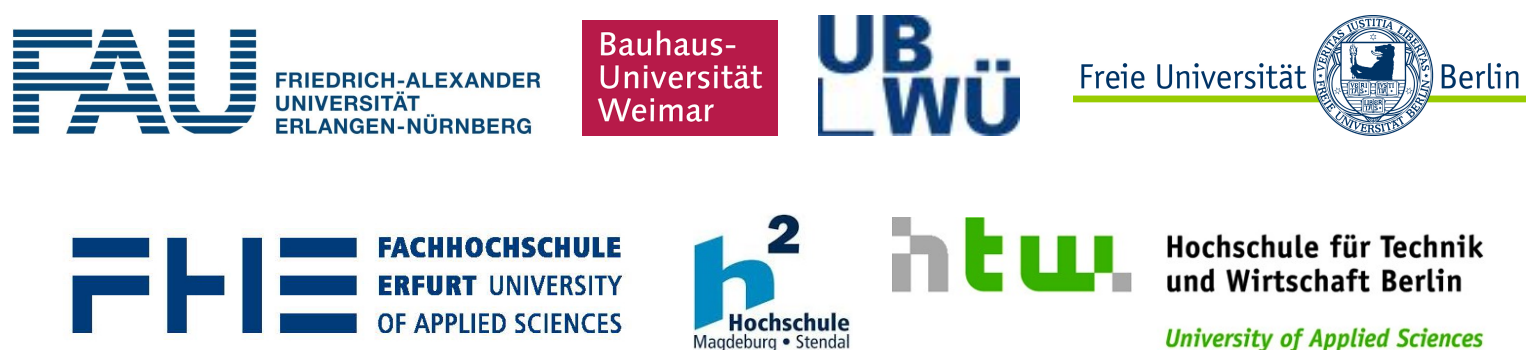

Hochschule für Technik und Wirtschaft Berlin

University of Applied Sciences
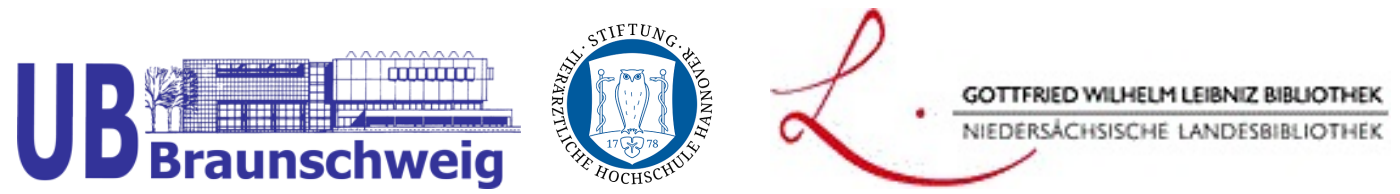


\section{EDITORIAL}

Estimadas y estimados lectores:

La filosofía de la Ciencia es un producto del siglo $\mathrm{XX}$, pero muchos autores contribuyeron de manera significativa para consolidar el concepto mismo de ciencia; comparto con ustedes algunos datos que nos permitirán fortalecer el actuar como investigadores.

Uno de los filósofos que tiene gran trascendencia hasta nuestros tiempos es Arquímedes quien nació en Siracusa - Sicilia, (287-212 a. C.), se le considera un genio excéntrico y muchos de sus inventos en la actualidad son el fundamento de muchos desarrollos científicos y tecnológicos.

En la segunda mitad del siglo XVI (1564-1630) Galileo Galilei quien fundamenta su actuar con una frase muy significativa: «Me parece que aquellos que solo se basan en argumentos de autoridad para mantener sus afirmaciones, sin buscar razones que las apoyen, actúan en forma absurda. Desearía poder cuestionar libremente y responder libremente sin adulaciones. Así se comporta aquel que persigue la verdad»; recurre siempre a la naturaleza para confirmar sus conclusiones deducidas previamente de un razonamiento a partir de hipótesis puramente teóricas, es el inicio del método científico de investigación.

En ese mismo siglo otro astrónomo y filósofo muy influyente, Johannes Kepler (1571-1630) con el descubrimiento de los movimientos planetarios define las llamadas leyes de Kepler; también realizó aportaciones en el campo de la óptica, enunció una primera aproximación satisfactoria de la ley de la refracción y analizó el aspecto geométrico de diversos sistemas ópticos.

En el siglo XVII Isaac Newton (1642-1727) realizó descubrimientos fundamentales en el campo de las matemáticas y la óptica, los aportes generados por este filósofo sustentan todo el análisis de transferencia de calor que da soporte al desarrollo de tecnología para el aprovechamiento de la energía térmica.

Descartes (1596-1650) manifiesta: «En nuestra búsqueda del camino directo a la verdad, no deberíamos ocuparnos de objetos de los que no podamos lograr una certidumbre similar a las de las demostraciones de la aritmética y la geometría», se requiere implantar procedimientos que garanticen confiabilidad de los datos medidos para dar confiabilidad a los modelos propuestos.

Claude Bernard (1813-1878) sostiene, entre otros, que la observación es la constatación pura y simple de los fenómenos naturales tal como se presenta a los sentidos, mientras que la experimentación es la constatación de los fenómenos creados por el experimentador.

El método experimental, en cuanto método científico, descansa por entero sobre la verificación experimental de una hipótesis científica.

Popper (1985) manifiesta que el método científico considera la formulación de ideas innovadoras y estas someterlas a las más razonables, rigurosas y eficaces refutaciones posibles; determina el método ensayo y error que consiste en proponer hipótesis audaces y exponerlas a las más severas críticas para tratar de determinar una equivocación si esta existiera.

Otro filósofo actual que ha generado grandes aportes en el método científico es Mario Bunge (2007), él explica que una tecnología eficiente se fundamenta en hipótesis de mecanismos bien confirmadas, para esto se requieren procesos que son parte de un sistema concreto al que denomina materialismo sistémico; al sistema lo denomina como un objeto complejo cuyas partes o componentes se mantienen unidos por medio de vínculos de algún tipo ya sean estos lógicos o materiales. Los puentes que se generan entre la teoría y el hecho se pueden describir como el método hipotético-deductivo en donde en primer lugar, se deducen las consecuencias observables de la teoría, luego se obtienen datos empíricos, estos se confrontan con predicciones y, finalmente, se evalúa la teoría, indica que contiene una importante parte de verdad, pero que también es gravemente defectuoso puesto que las teorías no implican observaciones así sin más y, en consecuencia, no pueden ser contrastados los datos de modo directo con los datos empíricos pertinentes. 
Determinación de la presión máxima de compresión de un MEP basado en una RNAR Determination of the maximum compression pressure of a MEP based on a RNAR. Wilmer Contreras, Mauricio Arichávala, Cristian Jérez

Caracterización de un generador de flujo axial para aplicaciones en energía eólica Characterization of an axial flow generator for applications in wind energy ......... Mauricio Carrillo-Rosero, Cristian Claudio-Medina, Alex Mayorga-Pardo

Análisis energético, exergético y económico de un sistema de cogeneración: Caso para una planta azucarera de San Pablo

Energetic, exergetic and economic enalysis of a cogeneration cystem: sugarcane

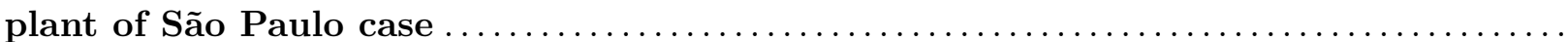

Omar R. Llerena P.

Análisis de medidas de ahorro de energía en una empresa de producción

Analysis of energy saving measures in a production company $\ldots \ldots \ldots \ldots \ldots \ldots \ldots$ Luisa Salazar, Víctor Guzmán, Alexander Bueno

Factores para seleccionar tuberías de conducción de gas licuado de petróleo en el Ecuador

Factors for selecting petroleum liquefied gas conduction pipes in Ecuador Diego Venegas, César Ayabaca, Santiago Celi, Juan Rocha, Euro Mena

Incentivo a la generación distribuida en el Ecuador Incentive pertaining to energy the generation distributed in Ecuador

Jorge Patricio Muñoz-Vizhñay, Marco Vinicio Rojas-Moncayo, Carlos Raúl Barreto-Calle

On the behaviour of spherical inclusions in a cylinder under tension loads Estudio del comportamiento de inclusiones esféricas en un cilindro bajo tracción..... Sebastián Montero, Roger Bustamante, Alejandro Ortiz-Bernardin

Comparación entre redes neuronales artificiales y regresión múltiple para la predicción de la rugosidad superficial en el torneado en seco

Comparison between artificial neural network and multiple regression for the prediction of superficial roughness in dry turning

Yoandrys Morales-Tamayo, Yusimit Zamora-Hernández, Paco Vásquez-Carrera,

Mario Porras-Vásconez, Joao Bárzaga-Quesada, Ringo López-Bustamante

Obtención de ACS con energía solar en el cantón Cuenca y análisis de la contaminación ambiental

Obtaining of SHW with solar energy in the canton Cuenca and analysis of environmental pollution

John Calle Siguencia, Óscar Tinoco Gómez

Cuantificación de la opacidad en motores electrónicos diésel usando diésel y biodiésel Quantification of opacity in electronic motors diesel using diesel and biodiesel....... Carlos Mafla Yépez, Rommel Imbaquingo Navarrete, Jorge Melo Obando, Ignacio Benavides Cevallos, Erik Hernández Rueda

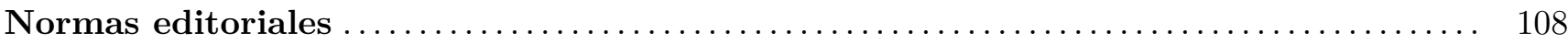

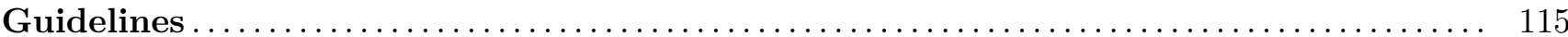




\title{
DETERMINACIÓN DE LA PRESIÓN MÁXIMA DE COMPRESIÓN DE UN MOTOR DE ENCENDIDO PROVOCADO BASADO EN UNA RED NEURONAL ARTIFICIAL RECURRENTE
}

\author{
DETERMINATION OF THE MAXIMUM \\ COMPRESSION PRESSURE OF AN ENGINE \\ PROVOKED IGNITION BASED ON A RECURRENT \\ ARTIFICIAL NEURAL NETWORK
}

\author{
Wilmer Contreras ${ }^{1, *}$, Mauricio Arichávala $^{1}$, Cristian Jérez $^{1}$
}

\section{Resumen}

En la presente investigación se realiza la explicación de la metodología aplicada a la determinación de la presión máxima de compresión de un motor de combustión interna alternativo de encendido provocado (MEP), el cual se basa en un estudio que parte de la caracterización de las curvas del consumo de amperaje del motor de arranque. Se aplica un protocolo de adquisición de datos y su posterior análisis estadístico. Los valores estadísticos de la señal como energía, promedio, desviación estándar, varianza, kurtosis, asimetría, máximo, mínimo y factor de cresta son seleccionados en función al mayor aporte de información para la caracterización del experimento; estos valores generan bases de datos las cuales son aplicadas para la creación y entrenamiento de una red neuronal artificial recurrente (RNAR) en la cual se obtiene un error absoluto menor al $2 \%$. En una primera instancia se aplica la metodología de pruebas en un motor ensamblado en un banco didáctico y luego se procede a la aplicación del método en motores aplicados en vehículos.

Palabras clave: diagnóstico, presión de compresión, RNAR, MEP, red Elman, capa recurrente.

\section{Abstract}

In the present research the explanation of the applied methodology for the determination of the maximum pressure of compression of an engine of alternative internal combustion of provoked ignition (EPI), that is based on a study that starts from the characterization of the curves of the amperage rating of the starter motor. A protocol for data acquisition and subsequent statistical analysis is applied. The statistical values of the signal as energy, average, standard deviation, variance, kurtosis, asymmetry, maximum, minimum and crest factor are selected in function of the greater contribution of information for the characterization of the experiment; these values generate databases that are applied for the creation and training of a recurrent artificial neural network (RANN) in which an absolute error of less than $2 \%$ is obtained. In the first instance, the test methodology is applied in an engine assembled in a didactic bank and then the application of the method is applied in vehicles.

Keywords: diagnosis, compression pressure, RANN, EPI, Elman Network Layer Appellant.

\footnotetext{
$\overline{1, *}$ Grupo de Investigación de Ingeniería del Transporte (GIIT), Carrera de Ingeniería Mecánica Automotriz, Universidad Politécnica Salesiana, Cuenca - Ecuador. Autor para correspondencia rcontreras@ups.edu.ec (D) http://orcid.org/0000-0003-2300-9457, (D) http://orcid.org/0000-0002-3111-7848,

(D) http://orcid.org/0000-0003-1474-5014.
} (enero-junio). pp. 9-18. DOI: https://doi.org/10.17163/ings.n19.2018.01. 


\section{Introducción}

En la actualidad, la reparación y diagnóstico aplicados en el área del transporte automotriz se basan desde esquemas artesanales hasta llegar a un alto nivel de tecnificación [1]. El incremento diario en el parque automotor demanda servicios especializados con la consiguiente reducción en los tiempos de detección y reparación de fallos. Esto ha traído el desarrollo de investigaciones mediante el análisis de vibraciones [2], para poder identificar los parámetros de operación crítica, análisis experimentales de la emisión acústica del motor alternativo [3], determinación de cilindrada del motor de manera no intrusiva [4].

Debido a la complejidad de variables que intervienen en el diagnóstico de los motores de combustión interna se hace necesaria la aplicación de matemática computacional.

La utilización de la técnica de redes neuronales es considerada de gran contribución en el análisis de los parámetros del motor de combustión interna, según expresa Saraswati y Chand; se puede reconstruir la presión del cilindro con el uso de una red neuronal recurrente (RNN) [5], en igual forma, Cay y Cicek en el 2012, indican que se puede predecir el consumo específico de combustible basado en parámetros como: el freno-motor, la potencia efectiva, la presión media efectiva y la temperatura del gas de escape del motor. Para esto se utilizó un modelo de ANN basado en el algoritmo estándar de retropropagación, con errores medios menores que 3,8 \% [6].

De acuerdo con Czarnigowski, es posible determinar el valor del avance de chispa, mediante un modelo de red neuronal inverso al del par efectivo, consiguiendo así la estabilización de velocidad en ralentí [7].

La investigación de $\mathrm{Wu}$, Huang y Chang propone un sistema de diagnóstico de fallas del MCI, basado en la presión del colector de admisión, utilizando la transformada discreta de Wavelet (DWT) y la aplicación de RNA. Este tipo de diagnóstico reduce el defecto convencional de depender demasiado de la experiencia de los técnicos [8]. Un estudio muy similar propuesto por Shatnawi y Al-khassaweneh, en donde, la señal de sonido emitida por el MCI es la fuente de información para establecer fallas, mediante la red neuronal de extensión (ENN), la cual mejora el rendimiento en comparación con las RNA [9].

Los esfuerzos para predecir estados futuros del motor también son de gran interés en el desarrollo tecnológico de los motores, así lo demuestra un estudio desarrollado por la Universidad de Michigan, en donde las RNA son usadas para predecir el comportamiento de la combustión de un motor de ignición por compresión de carga homogénea (HCCI), durante su funcionamiento transitorio [10].

Con la misma finalidad de predecir el rendimiento y las emisiones de escape bajo diferentes estrategias de la EGR, los investigadores Roy, Banerjee y Bose presentan un estudio que utiliza RNA, obteniendo como resultados coeficientes de correlación dentro del rango de 0,987-0,999 y un error absoluto en el rango de 1,1-4,57 \% [11]. Con el propósito de optimizar a las RNA, se puede utilizar estrategias paralelas como el filtro de estructura variable lisa (SVSF) usado para entrenar eficientemente las RNA, y en consecuencia se conoce como RNA basada en SVSF, que se utiliza nuevamente para la detección y clasificación de fallas del motor utilizando datos de vibración en el dominio del ángulo del cigüeñal [12].

También, se ha desarrollado mediante las RNA, un sistema de diagnóstico automatizado de los fallos de encendido en los MCI que consta de tres etapas: detección, localización e identificación de gravedad de fallos [13].

Los investigadores Chen y Randall entrenaron una RNA para el análisis del dominio del tiempo que utiliza las características paramétricas de las emisiones acústicas (AE), para detectar daños en las válvulas de los MCI [14].

Es de hacer notar que existen a nivel general muy pocos sistemas inteligentes enfocados al diagnóstico de fallas mecánicas en las que intervenga la presión de compresión del MEP, siendo así los sistemas expertos como DELTA, de General Electric Company [15], utilizado para la reparación de locomotoras diésel y eléctricas, otro ejemplo es el STEAMER [15], desarrollado por Navy Research Perssoner Development Center, diseñado para enseñar el funcionamiento de una planta de propulsión de vapor como las utilizadas en los buques impulsados por vapor y por último se puede mencionar el Proyecto Eolo CN-235, desarrollado por la empresa española Construcciones Aeronáuticas S. A., el cual es un sistema de enseñanza interactivo para pilotos y técnicos de mantenimiento de aviones, modelo CN-235.

A nivel comercial y académico existen diferentes software como Autodata el cual cuenta con fichas técnicas y tiempos de reparación estimados, códigos de averías, rutinas de reparación de diferentes marcas y modelos de automóviles, permitiendo al personal técnico realizar cualquier tipo de reparación con la desventaja de la subjetividad de los operarios en las tomas de decisiones basadas en la experiencia profesional de prueba y error manteniendo un sistema de diagnóstico incipiente que en muchos de los casos podría ocasionar reparaciones erróneas y deficientes para el automóvil [1].

Esta revisión bibliográfica es la que conlleva a la investigación de la generación de metodologías en las cuales se proyecta determinar la presión máxima de compresión en la cámara de combustión en motores de encendido provocado de una manera mínimamente intrusiva y de rápida realización. 


\section{Métodos y materiales}

En la presente sección se desarrollan las temáticas principales que tienen que ver con la selección de parámetros menos invasivos, la instrumentación del motor, el diseño del software, la toma de datos, la validación de las muestras, la creación y entrenamiento de una RNAR.

\subsection{Selección de parámetros menos invasivos}

Se toma en consideración como objetivo principal evitar la manipulación y desarmado de elementos que serían necesarios para acceder a las bujías de encendido e instalar un probador de fugas o un compresómetro, por lo que se plantean como opciones: la medición del parámetro de flujo másico de aire del motor, la instalación de un medidor de presión en el múltiple de admisión [16], la medición de la corriente de consumo del motor de arranque, la medición de la caída de voltaje de la batería; todas estas opciones están con la condición de arranque del motor durante un tiempo determinado.

\subsection{Instrumentación del motor}

En el apartado anterior se mencionan los parámetros de medición para el desarrollo del experimento, de los cuales se explica que para realizar la medición del flujo másico de entrada de aire se puede aplicar un sensor de hilo caliente tipo MAF, para la medición de la presión en el múltiple de admisión se puede usar un sensor tipo MAP, para la medición de la corriente de consumo del motor de arranque se puede emplear una pinza amperimétrica, para la determinación de la caída de voltaje se puede utilizar un partidor de tensión conectado directamente a los bornes de la batería, lo cual se observa en la Tabla 1 y Figura 1.

Tabla 1. Instrumentación del motor

\begin{tabular}{cc}
\hline Parámetros & Sensores \\
\hline Flujo másico de aire & MAF \\
Presión de admisión & MAP \\
Corriente & Pinza amperimétrica \\
Voltaje & Partidor de tensión \\
\hline
\end{tabular}

Para identificar el cilindro que está con una diferencia significativa en su valor de compresión, se aplica una pinza inductiva para registrar la chispa que corresponde al cilindro 1 y que según el orden de encendido se localiza el cilindro con mayor variación. Se indica que en el motor del banco de pruebas se registra la señal del sensor CMP para identificar cada uno de los cilindros.

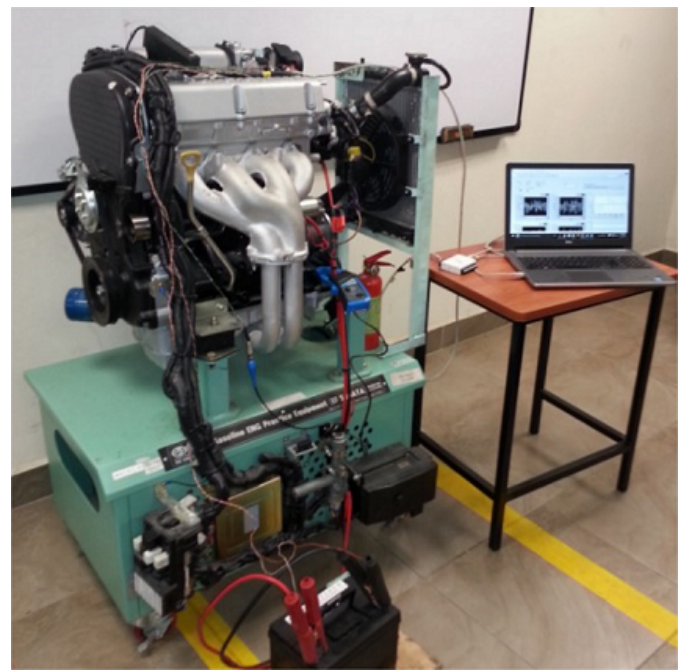

Figura 1. Instrumentación del motor.

Para la aplicación del sensor MAF se necesitan varios acoples que dependen del diámetro del colector de admisión por lo que esta opción se descarta siendo necesario, además, desmontar varios conductos del múltiple de admisión. Para la aplicación del MAP se debe identificar una toma en el múltiple de admisión la cual permita la conexión del sensor que, en algunos vehículos es inexistente, por lo tanto, se deben desconectar conductos pudiendo generar fallos mecánicos si estos no se reinstalan correctamente resultando en el descarte de esta opción.

La corriente de consumo del motor de arranque se puede medir con la instalación de una pinza amperimétrica, para lo cual no se requieren mayores exigencias, solo la identificación del cable y su instalación sobre este; la medición del voltaje se lo realiza directamente por la aplicación de pinzas en los bornes de la batería, indicado en la Figura 2, sin tener mayores complicaciones adoptando también esta opción.

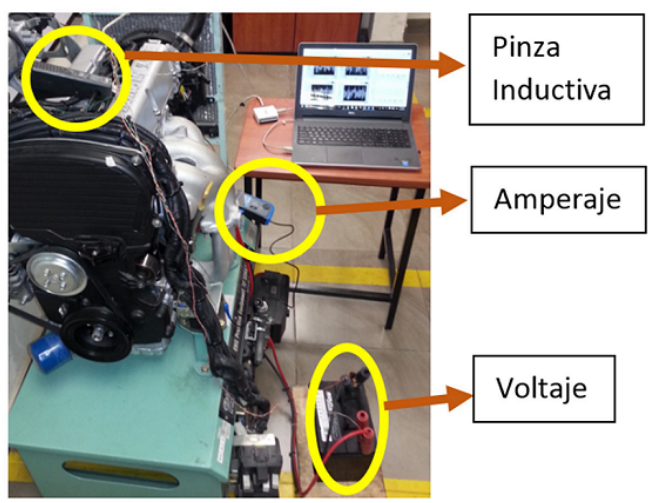

Figura 2. Medición de amperaje y voltaje de la batería.

En la Tabla 2 se resumen las características del motor a prueba y las de la pinza amperimétrica aplicada. 
Tabla 2. Equipos

\begin{tabular}{|c|c|}
\hline Gráfica & Características \\
\hline MEP & $\begin{array}{l}\text { Hyundai } \\
4 \text { cilindros } \\
\text { DOHC } \\
\text { Inyección electrónica (MFI) } \\
\text { VT = } 1997 \text { cc } \\
\text { Encendido por chispa / } \\
\text { Gasolina sin plomo } \\
\text { (RON 95) } \\
\text { Rc = 10.0 : } 1 \\
\text { MAP - DIS }\end{array}$ \\
\hline Hantek CC - 650 & $\begin{array}{l}\text { AC/DC Current Clamp } \\
\text { Bandwidth } 400 \mathrm{~Hz} \\
1 \mathrm{mV} / 10 \mathrm{~mA} \\
650 \mathrm{~A} \\
\mathrm{AC} / \mathrm{DC} \text { frecuency } \\
\text { range: Up to } 400 \mathrm{~Hz} \\
\text { Effective Measurement } \\
\text { range: } 20 \mathrm{~mA} \text { to } 650 \mathrm{~A} \mathrm{DC}\end{array}$ \\
\hline
\end{tabular}

\subsection{Diseño del software}

Al determinar que el consumo de amperaje es el parámetro menos invasivo junto con la medición de la caída de voltaje, se utiliza el software LabView, que es compatible con una tarjeta $\mathrm{Ni} 6009$, para la adquisición de datos a una tasa de $1 \mathrm{kHz}$, lo cual cumple con el criterio de Nyquist para el análisis de la señal.

También se indica que el software realiza la extracción de parámetros característicos y de estadística descriptiva de cada prueba realizada y que, además, genera una base de datos la cual posteriormente se aplicará en la creación y entrenamiento de una red neuronal artificial recurrente.

En las Figuras 3a, 3b y 3c se presenta una secuencia y parte de la programación aplicada para la adquisición, desarrollo del software y su entorno gráfico.

\subsection{Toma de datos}

Se muestran a continuación las gráficas de la toma de muestras del motor en estudio con diferencias en su presión de compresión (el motor se encuentra en condición de arranque).

En la Figura 4 se grafica la curva de presión de compresión en función al oscilograma de la curva de consumo de amperaje del motor de arranque, en la cual el motor se encuentra en condiciones estándares, es decir, todos sus cilindros con una presión de compresión estándar que es alrededor de 125 PSI.

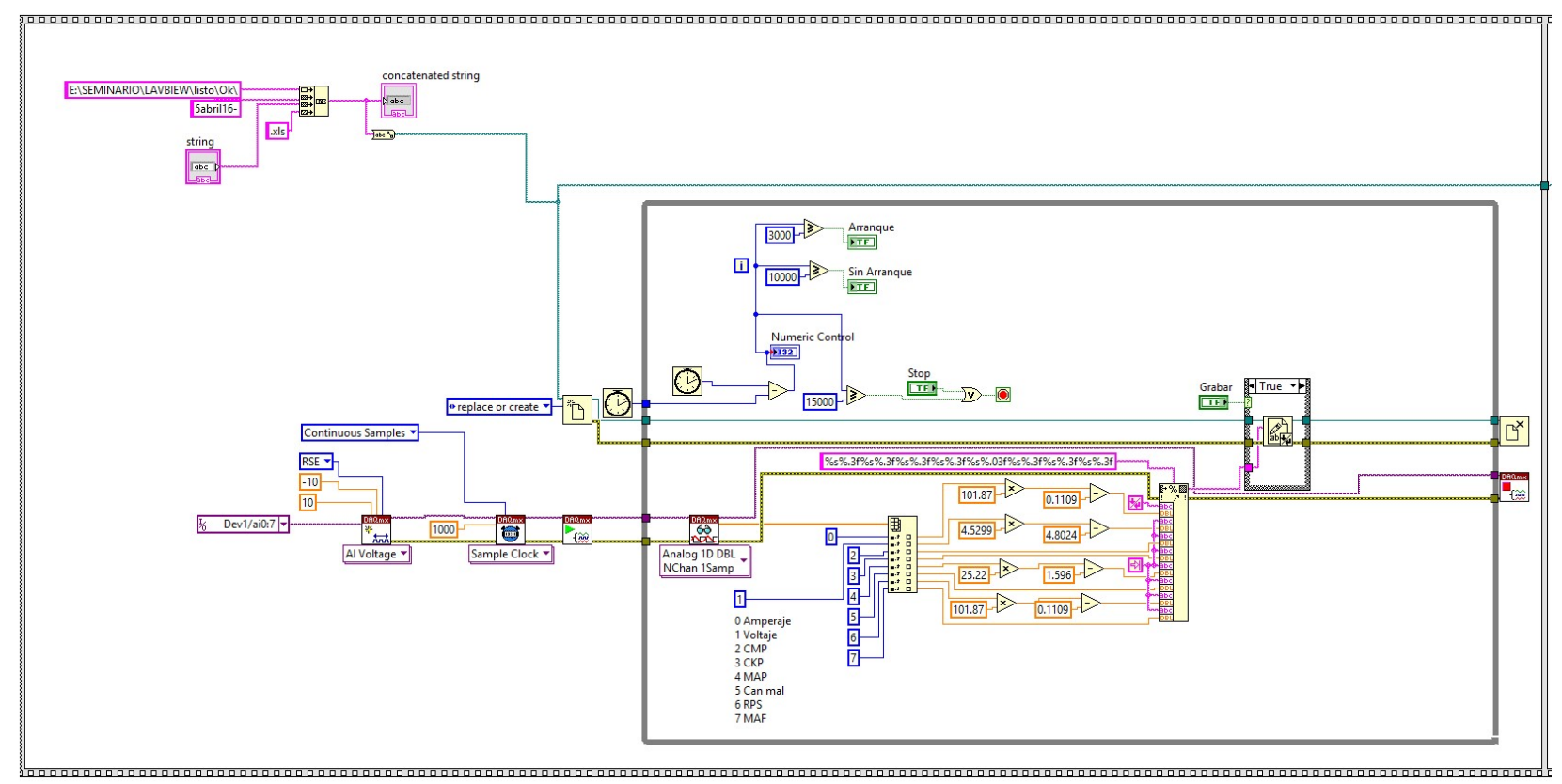

Figura 3a. Adquisición de las señales. 
Contreras et al. / Determinación de la presión máxima de compresión de un motor de encendido provocado basado en una red neuronal artificial recurrente

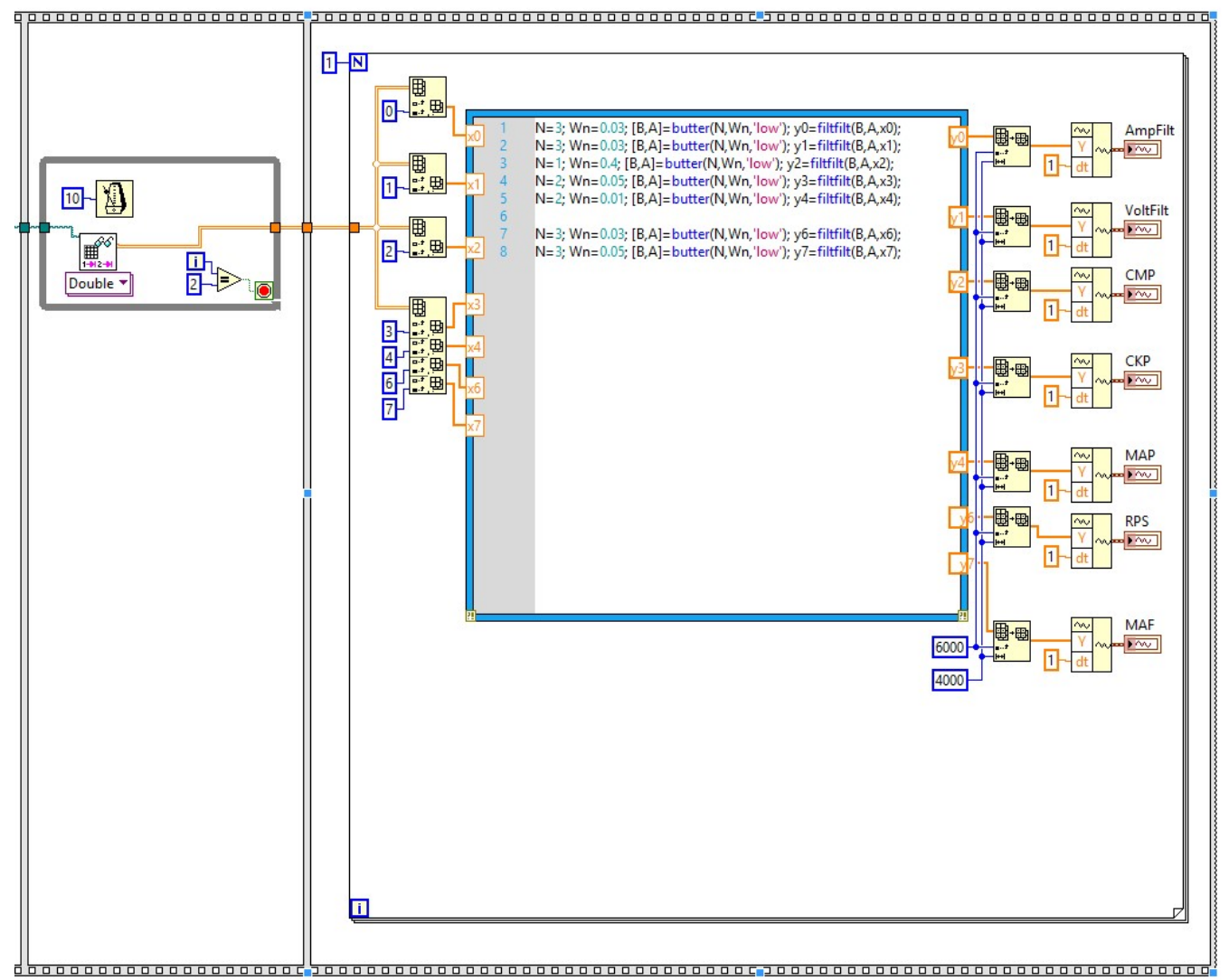

Figura 3b. Filtrado, desarrollo y extracción de las señales.

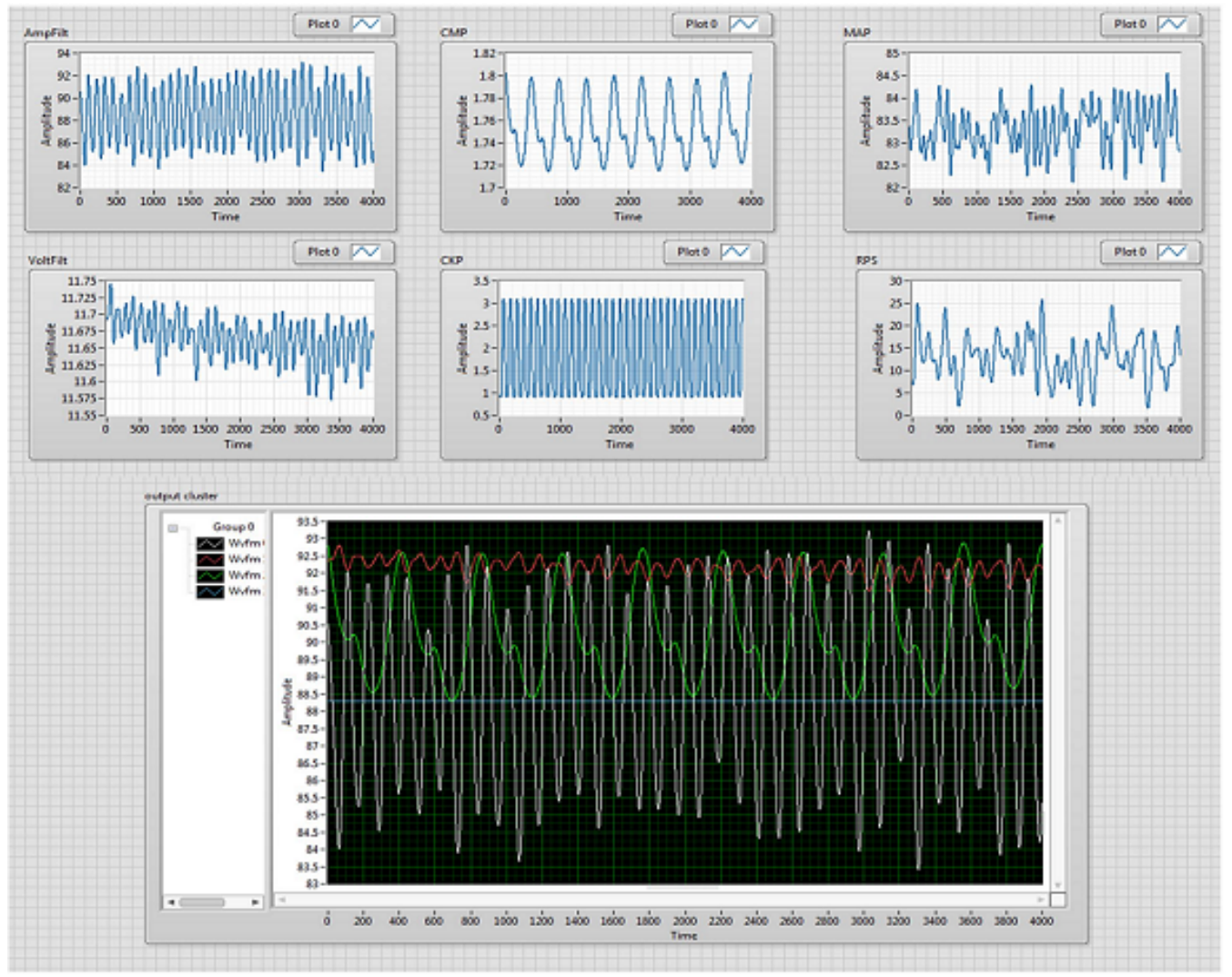

Figura 3c. Visualización de las señales y su entorno gráfico. 


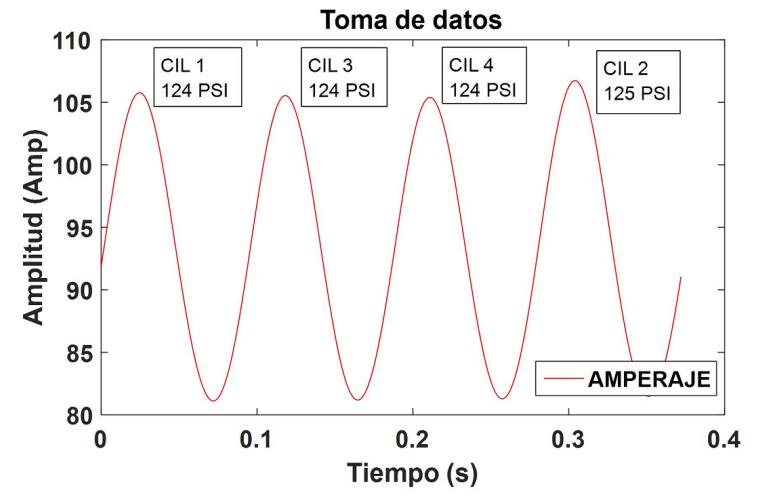

Figura 4. Toma de datos del motor sin mayor variación de compresión.

En las Figuras 5, 6, 7 y 8 se grafica la curva de presión de compresión en función al oscilograma de la curva de consumo de amperaje del motor de arranque, en las cuales el motor se encuentra con variaciones de compresión en cada cilindro, es decir, el cilindro correspondiente al orden de encendido tiene una presión de compresión de 90 PSI, mientras que los otros 3 cilindros restantes mantienen su presión estándar; además, se puede identificar la curva de la señal del sensor CMP.

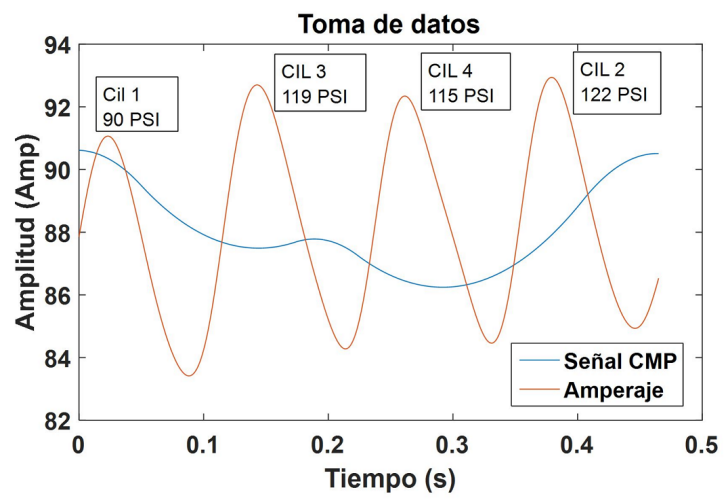

Figura 5. Toma de datos motor con variación de compresión en el cilindro 1.

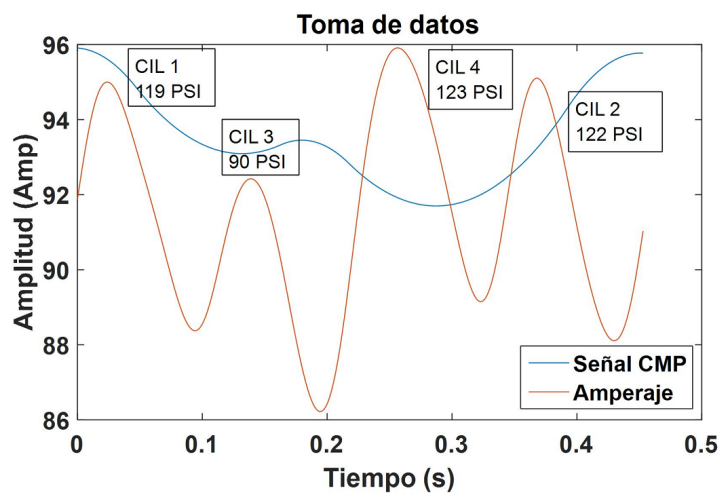

Figura 6. Toma de datos motor con variación de compresión en el cilindro 3.

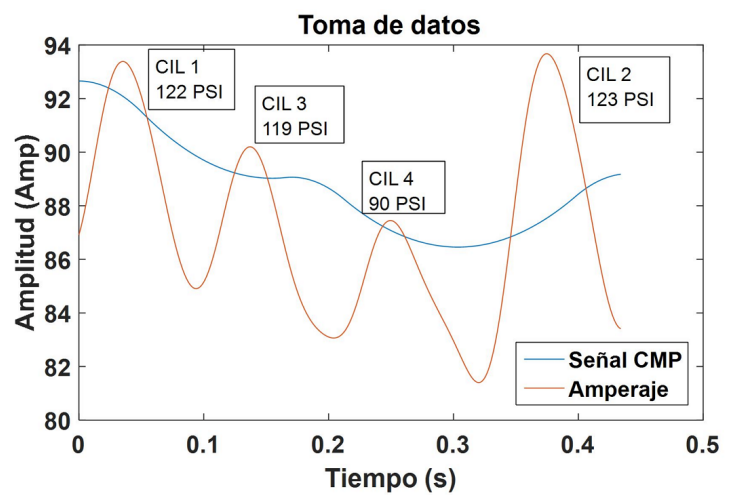

Figura 7. Toma de datos motor con variación de compresión en el cilindro 4 .

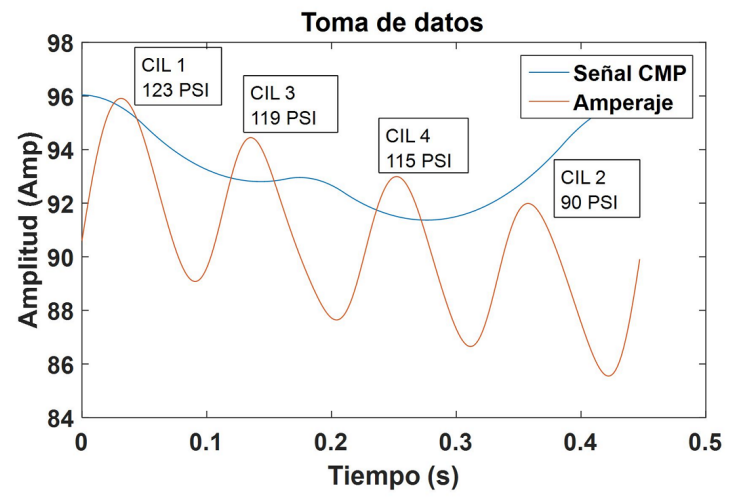

Figura 8. Toma de datos motor con variación de compresión en el cilindro 2 .

A continuación, en las Figuras 9, 10, 11 y 12 se aplica un diagrama de barras, en el cual cada barra representa el valor de la presión de compresión en función al consumo de amperaje del motor de arranque cuando el motor se encuentra con variaciones de la presión de compresión en cada cilindro, es decir, el cilindro correspondiente al orden 1-2-3-4, tienen una presión de compresión de 90 PSI, mientras que los otros 3 cilindros restantes mantienen su presión estándar.

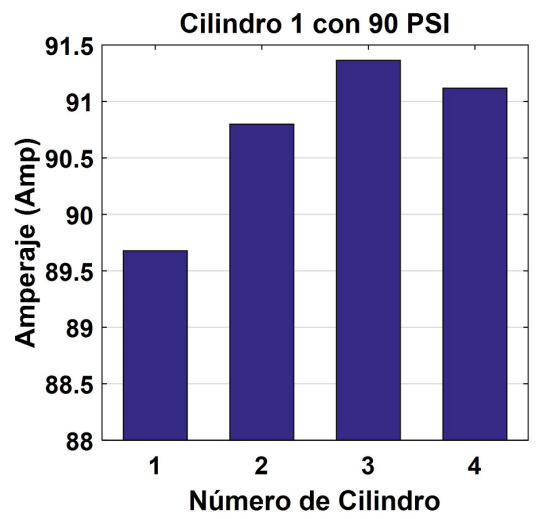

Figura 9. Motor con variación de compresión en el cilindro 1. 


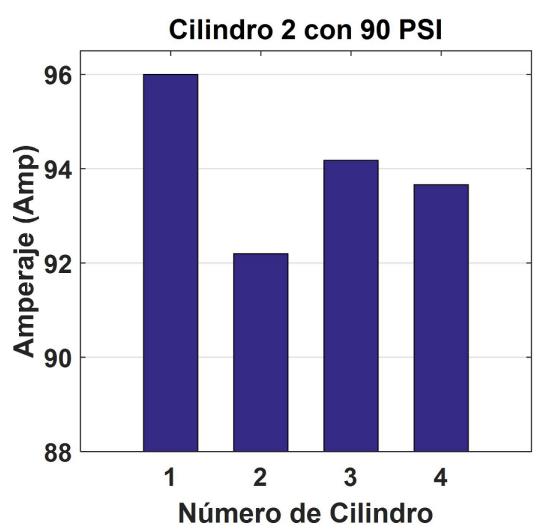

Figura 10. Motor con variación de compresión en el cilindro 2.

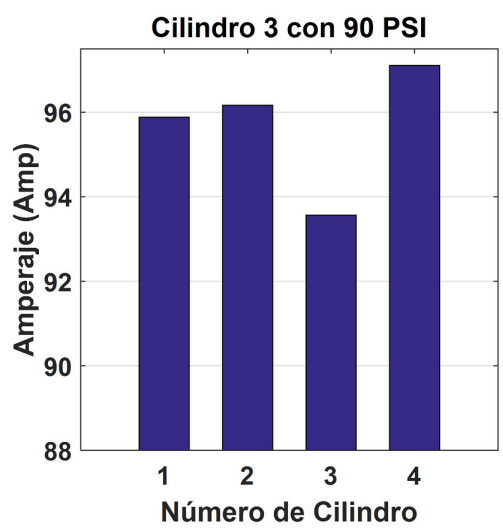

Figura 11. Motor con variación de compresión en el cilindro 3.

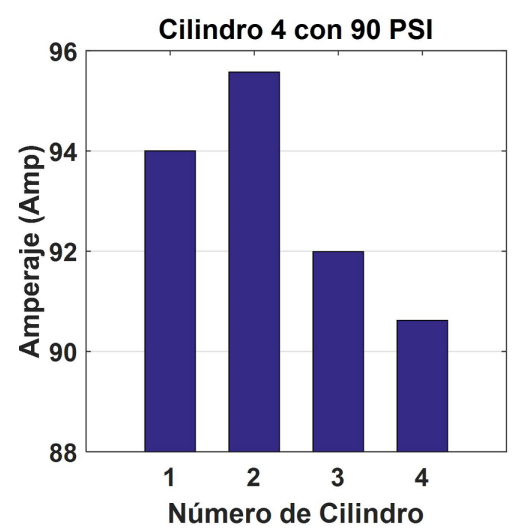

Figura 12. Motor con variación de compresión en el cilindro 4 .

\subsection{Validación de toma de muestras}

Las muestras tomadas por el software diseñado en la plataforma virtual de LabView son analizadas estadís- ticamente mediante un ANOVA en el cual tenemos los siguientes resultados.

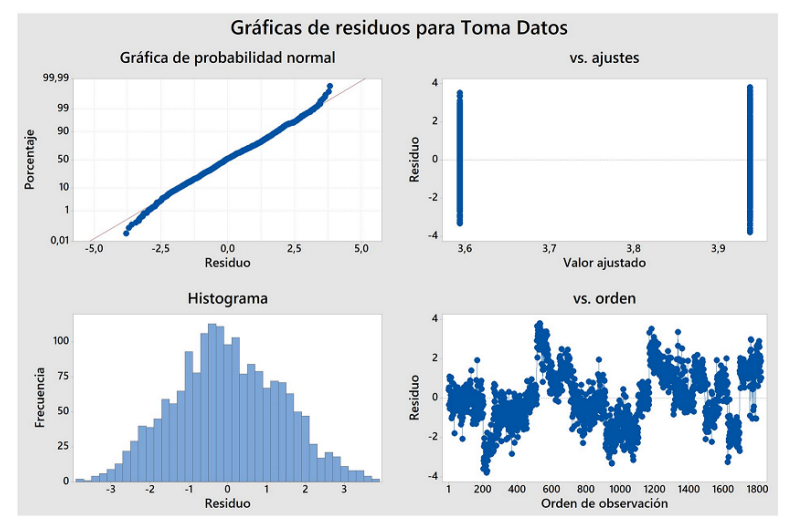

Figura 13. ANOVA de muestras.

La dispersión de puntos en el gráfico de residuos vs porcentaje mostrado en la Figura 13, tiende a ser una línea recta lo que afirma la normalidad de los datos y que, además, está confirmada por la distribución de valores en forma de la campana de Gauss en el histograma. Se valida el supuesto de varianza constante debido a que en la gráfica del valor ajustado vs residuo no se observa ningún patrón de acumulación de puntos; además, se corrobora que las tomas fueron de manera aleatoria ya que se observa que los valores en la gráfica de orden de observación vs residuo los puntos no muestran regiones de acumulación en la parte superior o inferior del cero, sino más bien estos fluctúan en un patrón aleatorio alrededor de la línea del cero.

En síntesis, la toma de datos es correcta y lo demuestra el resultado del ANOVA.

Para determinar los valores estadísticos característicos más significativos, se aplica un ANOVA unidireccional a las variables en estudio, con el cual se analizan los resultados del p-valúe, siendo el de menor valor el que revela la mayor significancia de las variables. A continuación, en la Tabla 3 se listan los valores estadísticos en orden de significancia basándose en el menor valor de $\rho$.

Tabla 3. Equipos

\begin{tabular}{cc}
\hline Valores estadísticos & $\boldsymbol{p}$-valúe \\
\hline Amp pico & 0,000 \\
Energía & 0,000 \\
Max & 0,000 \\
Media & 0,000 \\
Desviación estándar & 0,000 \\
Varianza & 0,000 \\
RMS & 0,000 \\
Asimetría & 0,000 \\
F. Cresta & 0,001 \\
Kurtosis & 0,003 \\
\hline
\end{tabular}




\subsection{Red neuronal tipo Elman}

Se aplica una red neuronal tipo Elman, esto basado en una corrida pre experimental en la cual se realizaron entrenamientos con diferentes tipos de redes como «feed-forward», «cascade-forward», «elman-forward», de las cuales se escoge la que menor error obtiene. Hecha la observación anterior, se indica que los parámetros de entrada son los valores característicos que resultan del análisis de la curva de consumo de amperaje del motor de arranque; estos se presentan en la Figura 14.

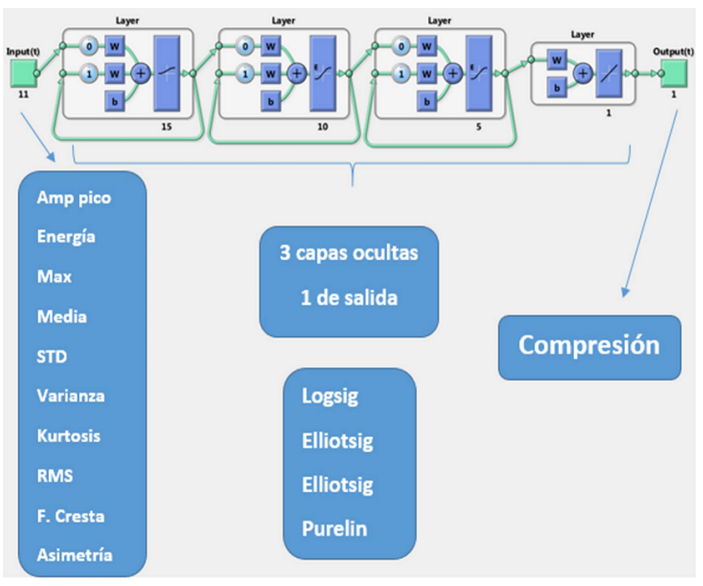

Figura 14. Red neuronal tipo Elman.

Se aplican 3 capas ocultas cada una con 15,10 y 5 neuronas por cada capa, debido al menor gasto computacional, ya que al aumentar capas y neuronas no disminuye el error y el tiempo de ejecución aumenta; para continuar se indica que las funciones de activación entre la neurona de entrada y la primera es de tipo Logsig, seguida en las dos capas de una función Elliotsig y por último entre la capa y la neurona de salida una función de tipo Purelin.

La neurona de salida indica el valor del resultado de la compresión, esto en función al análisis computacional que genera la RNAR tipo Elman.

El entrenamiento de la red se realiza con la función Levenberg-Marquardt (trainlm), la que se muestra en la Figura 15.

La Figura 16 indica el gradiente en la reducción del error cuadrático o MSE y el número de épocas o Epochs, que se realizan para el entrenamiento de la RNAR.

\section{Resultados y discusión}

A fin de comparar el correcto funcionamiento de la RNAR creada y entrenada del proceso propuesto, se realizan varias pruebas de diversos valores de compresión. En este apartado se presentan dos compresiones en concreto, que son valores que están alrededor de 120 PSI, en caso de motores con un funcionamiento correcto; otro caso en el que los valores se encuentran alrededor de 90 PSI lo que indica un fallo de desbalanceo en la combustión generalizada del motor.

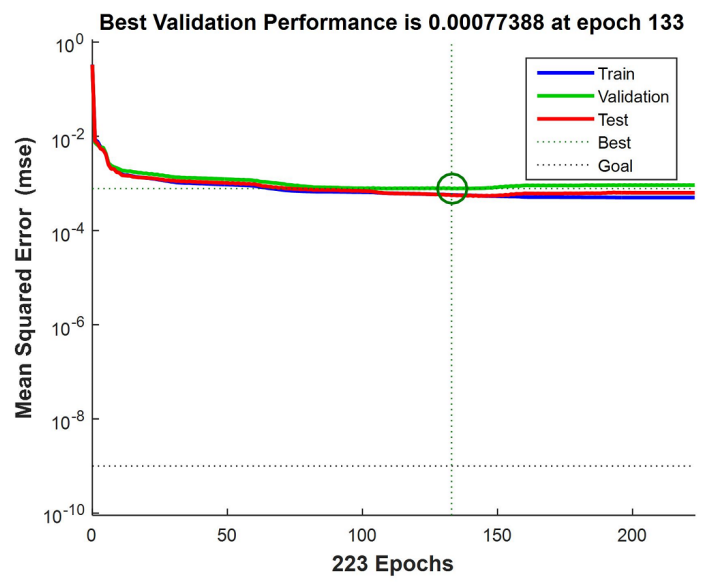

Figura 15. Entrenamiento de red tipo Elman.

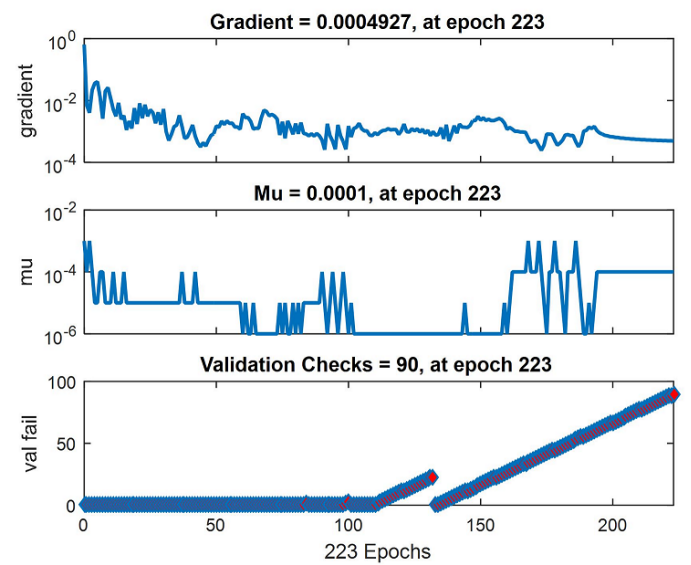

Figura 16. Evolución de entrenamiento de red tipo Elman.

En la Figura 17 se observa el resultado de los valores obtenidos por la RNAR para tomas de compresión de 120 PSI, donde el valor del promedio del error del resultado y el valor real es de $0.0895 \%$ del valor absoluto.

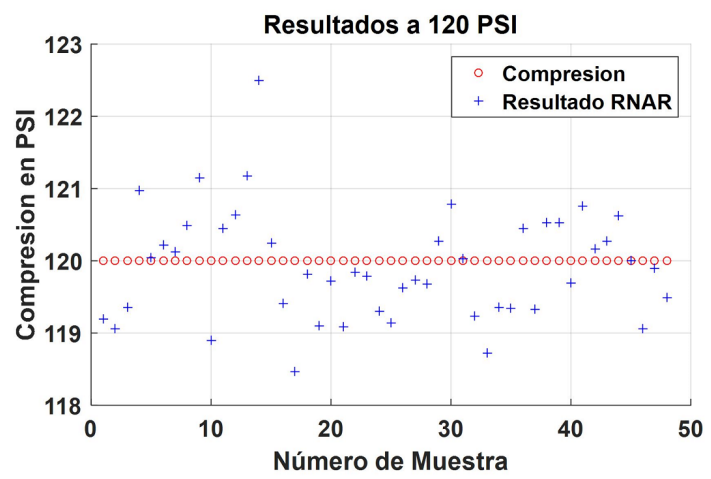

Figura 17. Resultados de compresión a 120 PSI. 
En la Figura 18, se evidencia el resultado de los valores obtenidos por la RNAR para tomas de compresión de 90 PSI, donde el valor del promedio del error del resultado y el valor real es de $0.2591 \%$ del valor absoluto.

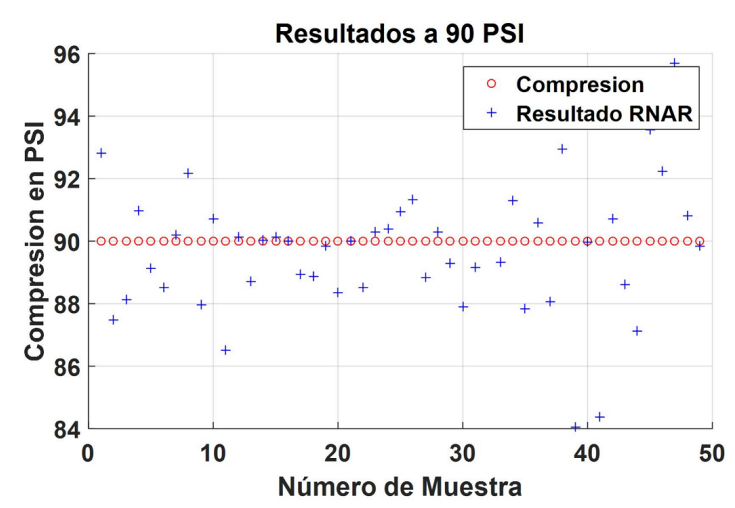

Figura 18. Resultados de compresión a 90 PSI.

\section{Conclusiones}

En este trabajo se muestra que la aplicación de redes neuronales artificiales recurrentes (RNAR) en la determinación de la compresión de un MEP constituye una alternativa claramente viable; además, presenta la ventaja de ser mínimamente invasiva con rangos de error menores al $1 \%$, pudiendo determinarse con un alto grado de probabilidad el valor de la compresión.

Otro aspecto fundamental a tener en cuenta es que el proceso de medición de compresión se aplica con mucha frecuencia en la evaluación de compraventa de vehículos, por lo tanto, esta metodología se presenta como una técnica altamente apropiada para ser integrada en un sistema de diagnóstico con la rapidez computacional que ofrecen las redes neuronales.

Tras la elaboración de este estudio en el cual se aplica una estructura de red neuronal tipo Elman, se ha observado que esta es la más apropiada dada la naturaleza dinámica de los patrones obtenidos por el análisis del consumo de corriente del motor de arranque.

\section{Referencias}

[1] R. F. Covarrubias and A. G. F. Covarrubias, "Desarrollo de un sistema experto para el diagnóstico de fallas automotrices." Revista Iberoamericana de Tecnología en Educación y Educación en Tecnología (TEEET), no. 11, pp. 83-91, 2013. [Online]. Available: https://goo.gl/Uz5g5y

[2] O. Criollo and H. Matute, "Diagnóstico de fallos en la combustión para motores de combustión interna alternativos diésel por análisis de vibraciones." Tesis de Grado, Universidad Politécnica Salesiana, 2014. [Online]. Available: https://goo.gl/24c27G

[3] J. García, "Diseño de una sala de pruebas para motores alternativos de combustión interna." in Trabajo final de carrera, Universidad Politécnica de Cataluña, 03 2007. [Online]. Available: https://goo.gl/xJv1ym

[4] W. Contreras and J. Fajardo, "Diseño y construcción de un sistema prototipo para determinar la cilindrada total de un motor ciclo otto por un método no invasivo mediante labview," Master's thesis, Escuela Politécnica Nacional, 2012. [Online]. Available: https://goo.gl/SdZ6ec

[5] S. Saraswati and S. Chand, "Reconstruction of cylinder pressure for si engine using recurrent neural network." Neural Computing \& Applications, vol. 19, no. 6, pp. 935-944, 2010. [Online]. Available: https://doi.org/10.1007/s00521-010-0420-6

[6] Y. Çay, A. Çiçek, F. Kara, and S. Sa` giroğlu, "Prediction of engine performance for an alternative fuel using artificial neural network," Applied Thermal Engineering, vol. 37, no. Supplement C, pp. 217-225, 2012. [Online]. Available: https: //doi.org/10.1016/j.applthermaleng.2011.11.019

[7] J. Czarnigowski, "A neural network modelbased observer for idle speed control of ignition in si engine," Engineering Applications of Artificial Intelligence, vol. 23, no. 1, pp. 1-7, 2010. [Online]. Available: https://doi.org/10.1016/j.engappai.2009.09.008

[8] J.-D. Wu, C.-K. Huang, Y.-W. Chang, and Y.-J. Shiao, "Fault diagnosis for internal combustion engines using intake manifold pressure and artificial neural network," $E x$ pert Systems with Applications, vol. 37, no. 2, pp. 949-958, 2010. [Online]. Available: https://doi.org/10.1016/j.eswa.2009.05.082

[9] Y. Shatnawi and M. Al-khassaweneh, "Fault diagnosis in internal combustion engines using extension neural network," IEEE Transactions on Industrial Electronics, vol. 61, no. 3, pp. 1434-1443, March 2014. [Online]. Available: https://doi.org/10.1109/TIE.2013.2261033

[10] V. M. Janakiraman, X. Nguyen, and D. Assanis, "Nonlinear identification of a gasoline hcci engine using neural networks coupled with principal component analysis," Applied Soft Computing, vol. 13, no. 5, pp. 2375-2389, 2013. [Online]. Available: https://doi.org/10.1016/j.asoc.2013.01.006 
[11] S. Roy, R. Banerjee, and P. K. Bose, "Performance and exhaust emissions prediction of a crdi assisted single cylinder diesel engine coupled with egr using artificial neural network," Applied Energy, vol. 119, Supplement C, pp. 330-340, 2014. [Online]. Available: https://doi.org/10.1016/j.apenergy.2014.01.044

[12] R. Ahmed, M. E. Sayed, S. A. Gadsden, J. Tjong, and S. Habibi, "Automotive internal-combustionengine fault detection and classification using artificial neural network techniques," IEEE Transactions on Vehicular Technology, vol. 64, no. 1, pp. 21-33, Jan 2015. [Online]. Available: https://doi.org/10.1109/TVT.2014.2317736

[13] J. Chen and R. B. Randall, "Improved automated diagnosis of misfire in internal combustion engines based on simulation models," Mechanical
Systems and Signal Processing, vol. 64-65, Supplement C, pp. 58-83, 2015. [Online]. Available: https://doi.org/10.1016/j.ymssp.2015.02.027

[14] S. M. Jafari, H. Mehdigholi, and M. Behzad, "Valve fault diagnosis in internal combustion engines using acoustic emission and artificial neural network," Shock and Vibration, vol. 2014, pp. 1-9, 2014. [Online]. Available: http://dx.doi.org/10.1155/2014/823514

[15] J. Giarratano and G. Riley, Sistemas expertos, principios y programación., T. Ed, Ed., 2004.

[16] M. Khajavi, S. Nasiri, and A. Eslami, "Combined fault detection and classification of internal combustion engine using neural network," Journal of Vibroengineering, vol. 16, no. 8, pp. 3912-3921, 2014. [Online]. Available: https://goo.gl/aWc9oW 


\title{
CARACTERIZACIÓN DE UN GENERADOR DE FLUJO AXIAL PARA APLICACIONES EN ENERGÍA EÓLICA
}

\section{Characterization OF AN AXIAL FLOW GENERATOR FOR APPLICATIONS IN WIND ENERGY}

\author{
Mauricio Carrillo-Rosero ${ }^{1, *}$, Cristian Claudio-Medina ${ }^{1}$, Alex Mayorga-Pardo $^{1}$
}

\section{Resumen}

El aprovechamiento de los recursos energéticos no convencionales en los últimos años se ha desarrollado principalmente con la intención de disminuir el uso de combustibles. A mediana y pequeña escala la tendencia mundial, cuando se habla de generación eléctrica utilizando la energía eólica, es el uso de generadores de imanes permanentes. Los generadores de flujo axial son lo que se encuentran con mayor frecuencia a disposición. La principal característica de estos generadores es su capacidad de entregar energía eléctrica a bajas revoluciones, con una eficiencia aceptable. En este proyecto de investigación se caracterizaron dos tipos de generadores de flujo axial; uno con bobinas en serie y otro con bobinas en paralelo, con la intención de valorar el rendimiento de cada uno. El diseño parte de la determinación del flujo magnético, número de revoluciones, número de polos, número de bobinas, voltaje de salida y pérdidas eléctricas, para luego realizar el contraste a través de la toma de datos de amperaje y voltaje con respecto al cálculo teórico; la finalidad es establecer la eficiencia que presentan cada una de las configuraciones. Se estableció que el generador en serie posee características que lo convierten en una configuración que tiene una eficiencia mayor que la del generador en paralelo.

Palabras clave: generadores, flujo axial, imanes permanentes, energía eólica, eficiencia.

\section{Abstract}

The use of unconventional energy resources in recent years has developed mainly with the intention of reducing the use of fossil fuels to obtain electricity. When talking about electricity generation at medium and small scale, the global trend while taking advantage of wind energy, is the use of permanent magnet generators. Axial flow generators are the most often available: the main feature of these generators is their ability to deliver electrical power at low revolutions, with an acceptable efficiency. In this research project two types of axial flow generators were characterized; one with coils in series and another with coils in parallel, with the intention of evaluating the performance of each one. The design starts from the determination of the magnetic flux, number of revolutions, number of poles, number of coils, output voltage and electrical losses, to then carry out the contrast through the data acquisition of amperage and voltage with respect to the theoretical calculation; the purpose is to establish the efficiency of each of the configurations. It was established that the series generator has characteristics that make it a configuration that has an efficiency greater than that of the generator in parallel.

Keywords: Generators, flow, axial, energy, wind, efficiency.

\footnotetext{
1,*Facultad de Ingeniería Civil y Mecánica, Universidad Técnica de Ambato - Ecuador. Autor para correspondencia cmcarrillo@uta.edu.ec. (1) http://orcid.org/0000-0001-6578-9362,

(D) http://orcid.org/0000-0001-7091-3516, (Dhttp://orcid.org/0000-0001-8875-8470.
}

Recibido: 11-05-2017, aprobado tras revisión: 27-10-2017

Forma sugerida de citación: Carrillo, M.; Claudio, C.; Mayorga, A. (2018). «Caracterización de un generador de flujo axial para aplicaciones en energía eólica». Ingenius. N. ${ }^{\circ} 19$, (enero-junio). pp. 19-28. Dor: https://doi.org/10.17163/ ings.n19.2018.02. 


\section{Introducción}

La demanda creciente de combustibles fósiles, junto a la disminución de sus reservas, ha desatado un continuo aumento de los precios que afecta a la economía mundial. Además, el uso de estos combustibles produce gases, como el dióxido de carbono $\left(\mathrm{CO}_{2}\right)$, cuyo efecto invernadero provoca el incremento de la temperatura de la superficie del planeta [1].

Según la WWEA en su boletín informativo de septiembre de 2016, manifiesta que, a finales de 2014, la capacidad instalada de viento a pequeña escala, registró más de $830 \mathrm{MW}$ a nivel mundial. Esto representa una tasa de crecimiento del 10,9\% sobre 2013, cuando se registraron $749 \mathrm{MW}$. El año 2013 fue un 10,3\% superior al total de $678 \mathrm{MW}$ instalados en 2012. China representa el $41 \%$ de la capacidad global, los Estados Unidos el $30 \%$ y el Reino Unido el $15 \%$. A finales de 2014, un total acumulado de al menos 945000 pequeños aerogeneradores se instalaron en todo el mundo. Se trata de un aumento del 8,3\% (7,4 $\%$ en 2013) en comparación con el año anterior, cuando se registraron 872000 unidades [2]. Se estima que la capacidad en la industria mini-eólica para el año 2020 podría suministrar $50000 \mathrm{MW}$ en todo el planeta [1].

Para ayudar a reducir costos y minimizar el mantenimiento tanto como sea posible, muchos de los desarrolladores de turbinas eólicas emplean generadores de imanes permanentes (GIP) de impulsión directa (sin caja de engranajes) mientras tengan un diseño de generador mecánicamente simple. Se pueden utilizar varias topologías de generador de imanes permanentes [3].

Un diseño adecuado para un generador con flujo axial de estator central, se basa en dos piezas rotóricas que se ubican lateralmente solidarias al eje de la máquina. Esta configuración de dos entrehierros tiene la ventaja de cancelar las fuerzas longitudinales sobre el estator, además, esta topología minimiza la inductancia de dispersión. [4]

El presente proyecto tiene como objetivo la caracterización de GIP de flujo axial en dos configuraciones de bobinas, una en serie y otra en paralelo utilizando imanes de hierro-boro-neodimio, para determinar la eficiencia de cada una de ellas.

\subsection{Características del diseño de un generador de flujo axial}

Consiste en un par de discos delgados de hierro que giran alrededor de un eje perpendicular al mismo, y en cuyo contorno se sitúan un conjunto de imanes, los cuales crean un campo magnético paralelo al eje de giro como se muestra en la Figura 1. La disposición axial para el corte del campo magnético es paralela respecto al embobinado, esta clase de disposición permite una excitación de los electrones sin fricción y oposición magnética, el corte que se realiza es de $180^{\circ}$ produciendo menores pérdidas que la generación radial [5].

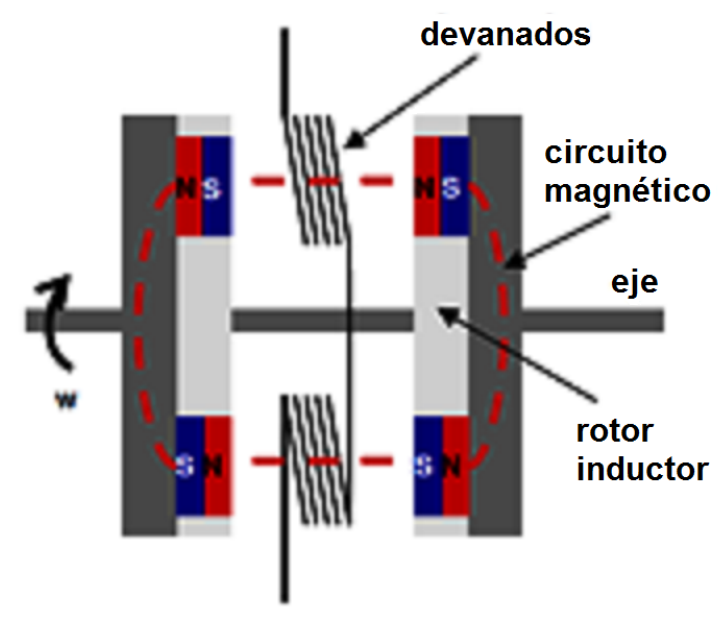

Figura 1. Vista transversal de un generador axial.

\subsection{Bobinas con núcleo de aire}

El conductor se enrolla sobre un soporte hueco y posteriormente se retira este quedando con un aspecto parecido al de un muelle. Se utiliza en frecuencias elevadas. Una variante de la bobina anterior se denomina solenoide y difiere en el aislamiento de las espiras y la presencia de un soporte que no necesariamente tiene que ser cilíndrico. Se utiliza cuando se precisan muchas espiras. Estas bobinas pueden tener tomas intermedias, en este caso se pueden considerar como dos o más bobinas sobre un mismo soporte y conectadas en serie.

Cada bobina produce un voltaje en función de cómo se conecten, que pueden ser de dos tipos: en serie y en paralelo. La conexión en serie consiste en unir el final de la primera bobina con el principio de la siguiente para que los voltajes se sumen, por otro lado, para conexión en paralelo los inicios se conectan entre sí y el voltaje resultante es el mismo que el de una bobina, con la diferencia de que se puede conseguir el doble de corriente. En paralelo es inevitable que el voltaje de salida de cada bobina sea ligeramente diferente lo que conduce a que se generen corrientes parásitas que desperdician energía [6].

En la Figura 2 se observa la conexión de tres bobinas en serie para cada fase y el estator se compone por nueve bobinas en total, por lo tanto, es un generador trifásico. Una ventaja notable de este generador es que para el rectificador solo se tienen tres cables [6]. 


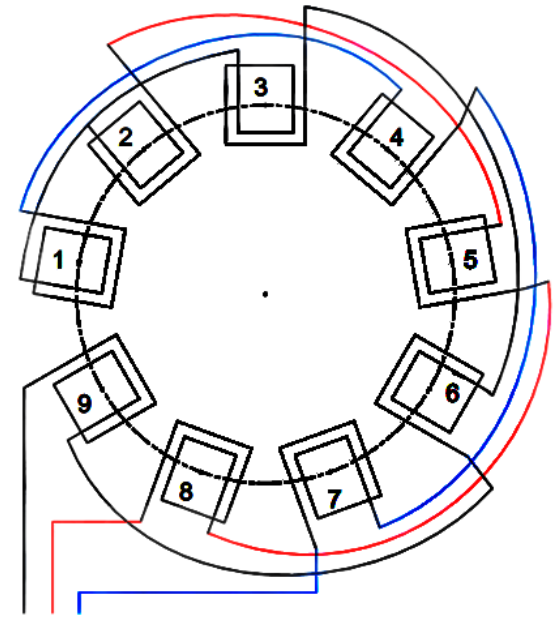

Figura 2. Esquema de conexión de bobinas en serie.

En la Figura 3 se tienen diez cables, cada uno conectado en paralelo a su respectiva bobina; el número de cables de salida determina la ubicación del rectificador ya que si se tienen demasiados cables el movimiento del generador en el extremo de la torre queda limitado, lo que implica que el rectificador debe estar ubicado junto al generador y esto ocasiona problemas de mantenimiento; el número de fases no es un problema para la rectificación ya que se pueden variar las conexiones de los puentes para rectificar hasta dos fases por cada uno $[6]$.

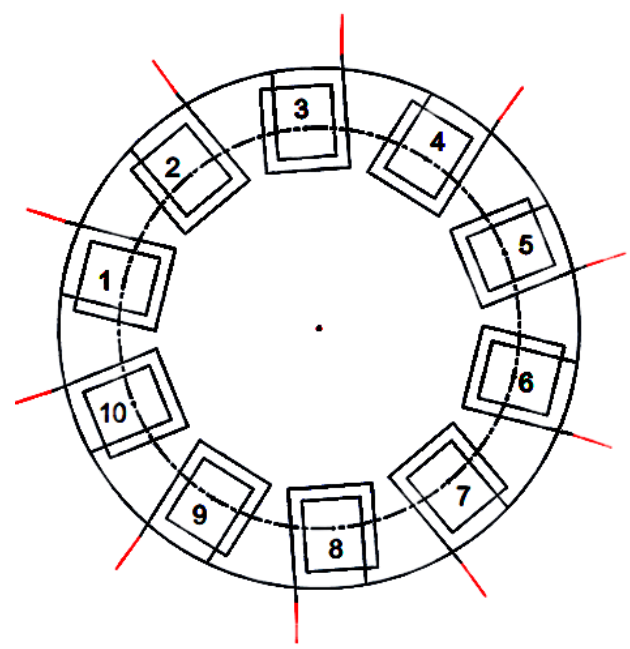

Figura 3. Esquema de conexión de bobinas en paralelo.

\subsection{Imanes permanentes}

El compuesto básico en los imanes permanentes utilizados en la investigación es en base de neodimio-hierroboro $(\mathrm{NdFeB})$ que tiene baja resistencia a la corrosión por su recubrimiento con capas finas de níquel y cromo para aislar al material base del ambiente, subsanando la desventaja de desmagnetizarse a temperaturas menores como otros compuestos [7]. Se requieren imanes con un alto grado de magnetización, que hagan posible la ubicación de varios imanes en cada rotor $[8,9]$; además, la dirección de su polarización debe ser pertinente para esta aplicación; por lo que se seleccionaron imanes de neodimio grado 40 (N40)

\section{Materiales y métodos}

En la Figura 4 se muestra el esquema que permite determinar la densidad de flujo magnético a través de la Ecuación 1, aplicable a imanes rectangulares [10].

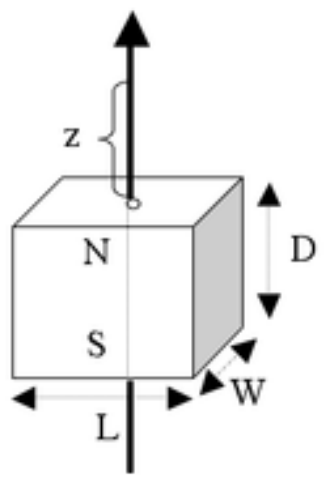

Figura 4. Esquema para la aplicación de la fórmula densidad de flujo.

Se determina la densidad de flujo magnético como:

$$
\begin{aligned}
B & =\frac{B r}{\pi}\left[\tan ^{-1}\left(\frac{L W}{2 z \sqrt{4 z^{2}+L^{2}+W^{2}}}\right)\right. \\
& \left.-\tan ^{-1}\left(\frac{L W}{2(D+z) \sqrt{4(D+z)^{2}+L^{2}+W^{2}}}\right)\right]
\end{aligned}
$$

$\mathrm{Br}=$ Campo remanente independiente de la geometría del imán $(1,26 \mathrm{~T}$, que se obtiene de los datos físicos del imán).

$\mathrm{z}=$ Distancia sobre el eje de simetría de una superficie polar $(10,5 \mathrm{~mm}$ que es la mitad de la distancia de los imanes en cada rotor a la que están separados los imanes).

$\mathrm{L}=$ Longitud del paralelepípedo $(46 \mathrm{~mm})$.

$\mathrm{D}=$ Espesor del paralelepípedo $(10 \mathrm{~mm})$.

$\mathrm{W}=$ Ancho del paralelepípedo $(30 \mathrm{~mm})$.

Sustituyendo los datos en la ecuación se tiene:

$$
B=0,137 T
$$

Para comprobar los datos calculados se realizaron simulaciones en el software FEMM como se muestra en la Figura 5. 


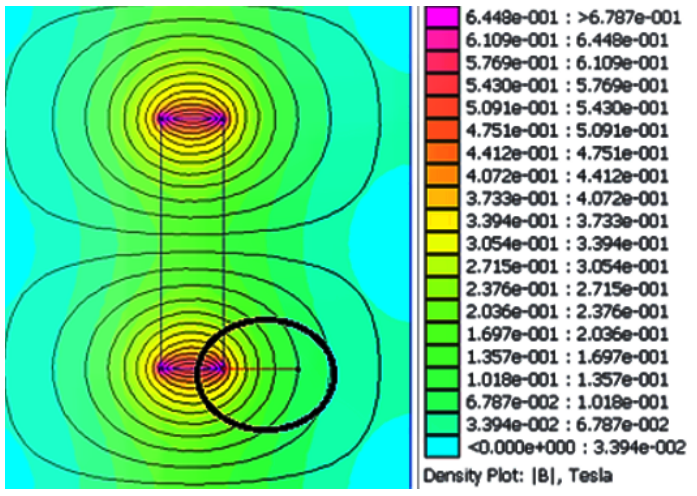

Figura 5. Simulación de la densidad de flujo de un imán vista lateral.

En el software se obtiene 0,1357 T que es muy cercano al valor calculado y se lo utiliza para el diseño. Al agregarle una placa de acero de bajo carbono se puede observar en la Figura 6, que los valores de flujo prácticamente se duplican por el efecto de concentración y aislamiento magnético que poseen los aceros.
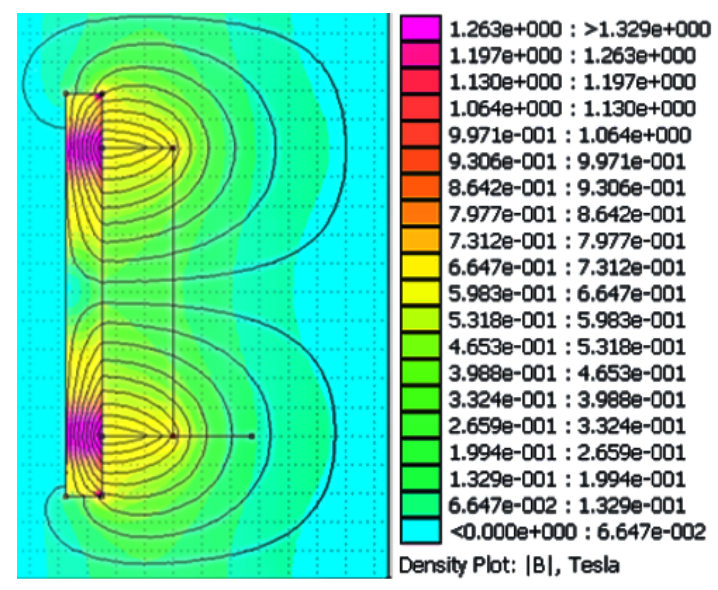

Figura 6. Simulación de la densidad de flujo de un imán pegado a una placa de acero, vista lateral.

Finalmente, se analiza el sistema de dos placas con imanes enfrentados como se muestra en la Figura 7, para obtener el flujo total.

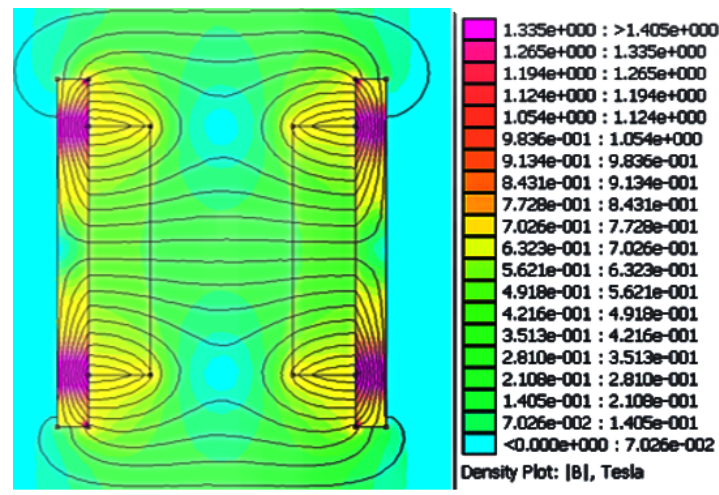

Figura 7. Simulación de la densidad de flujo de dos placas con imanes enfrentados, vista lateral.
Se tiene que el flujo entre las placas es de 0,5621 $\mathrm{T}$ (aproximadamente la mitad del flujo remanente) por lo cual queda confirmada la regla que propone [6]: «Si hay imanes en ambos discos y el hueco entre ellos es más o menos igual a la longitud combinada de los imanes, B será la mitad del flujo remanente».

\subsection{Determinación del número de revolu- ciones para el funcionamiento}

Para determinar este valor es necesaria la razón de velocidad de punta que es el índice de la velocidad rotacional de las palas respecto a la velocidad del viento como se indica en la ecuación (2).

$$
\lambda=\frac{\omega r}{v}
$$

Donde:

$\lambda=$ Razón de velocidad de punta (se utilizará un valor recomendado de 7 para todos los cálculos).

$\omega=$ Velocidad angular $(\mathrm{rad} / \mathrm{s})$.

$\mathrm{r}=$ Radio del aspa $(\mathrm{m})$ (se utiliza un valor de 1,5 para todos los cálculos).

$\mathrm{v}=$ Velocidad de viento $(\mathrm{m} / \mathrm{s})$

Para determinar la velocidad en rpm se utiliza la ecuación (3) [11].

$$
N=\frac{30 \lambda v}{r \pi}
$$

\subsection{Determinación del número de polos}

De acuerdo con [12] el número de polos se calcula con la ecuación (4) siendo f la frecuencia eléctrica:

$$
p=\frac{4 \cdot f \cdot r \cdot \pi}{\lambda \cdot v}
$$

Utilizando la Ecuación (4) se obtiene la Tabla 1, fijando como frecuencia máxima $60 \mathrm{~Hz}$ disponible en la red eléctrica.

De la Tabla 1 se desprende que mientras mayor sea la frecuencia más polos se necesitan; además, si la velocidad de viento es baja se requiere un mayor número de polos para alcanzar la frecuencia requerida. Tomando en cuenta la recomendación de [6] se elige un número de 24 polos (12 en cada rotor) con el que se garantiza una adecuada distribución de los imanes en los rotores. El valor de la frecuencia de funcionamiento no tiene por qué ser alto ya que en la rectificación se obtiene corriente directa que carece de frecuencia. 
Tabla 1. Datos físicos de los imanes

\begin{tabular}{|c|c|c|c|c|c|}
\hline \multicolumn{2}{|c|}{ V. de $2 \mathrm{~m} / \mathrm{s}$} & \multicolumn{2}{|c|}{ V. de $4 \mathrm{~m} / \mathrm{s}$} & \multicolumn{2}{|c|}{ V. de $6 \mathrm{~m} / \mathrm{s}$} \\
\hline $\begin{array}{l}\text { Frec. } \\
(\mathrm{Hz})\end{array}$ & $\begin{array}{l}\# \text { de } \\
\text { polos }\end{array}$ & $\begin{array}{l}\text { Frec. } \\
(\mathrm{Hz})\end{array}$ & $\begin{array}{l}\text { \# de } \\
\text { polos }\end{array}$ & $\begin{array}{c}\text { Frec. } \\
(\mathrm{Hz})\end{array}$ & $\begin{array}{l}\# \text { de } \\
\text { polos }\end{array}$ \\
\hline 10 & 13,46 & 10 & 6,73 & 10 & 4,49 \\
\hline 20 & 26,93 & 20 & 13,46 & 20 & 8,98 \\
\hline 30 & 40,39 & 30 & 20,2 & 30 & 13,46 \\
\hline 40 & 53,86 & 40 & 26,93 & 40 & 17,95 \\
\hline 50 & 67,32 & 50 & 33,66 & 50 & 22,44 \\
\hline 60 & 80,78 & 60 & 40,39 & 60 & 26,93 \\
\hline \multicolumn{2}{|c|}{ V. de $8 \mathrm{~m} / \mathrm{s}$} & \multicolumn{2}{|c|}{$\mathrm{V}$. de $10 \mathrm{~m} / \mathrm{s}$} & \multicolumn{2}{|c|}{ V. de $12 \mathrm{~m} / \mathrm{s}$} \\
\hline $\begin{array}{l}\text { Frec. } \\
(\mathrm{Hz})\end{array}$ & $\begin{array}{l}\# \text { de } \\
\text { polos }\end{array}$ & $\begin{array}{l}\text { Frec. } \\
(\mathrm{Hz})\end{array}$ & $\begin{array}{l}\# \text { de } \\
\text { polos }\end{array}$ & $\begin{array}{l}\text { Frec. } \\
(\mathrm{Hz})\end{array}$ & $\begin{array}{l}\# \text { de } \\
\text { polos }\end{array}$ \\
\hline 10 & 3,37 & 10 & 2,69 & 10 & 2,24 \\
\hline 20 & 6,73 & 20 & 5,39 & 20 & 4,49 \\
\hline 30 & 10,1 & 30 & 8,08 & 30 & 6,73 \\
\hline 40 & 13,46 & 40 & 10,77 & 40 & 8,98 \\
\hline 50 & 16,83 & 50 & 13,46 & 50 & 11,22 \\
\hline 60 & 20,2 & 60 & 16,16 & 60 & 13,46 \\
\hline
\end{tabular}

\subsection{Determinación del número de bobinas}

De acuerdo con [6] el número de bobinas está en función de los polos disponibles:

$$
N_{B}=\frac{3 N_{p}}{4}
$$

Donde:

$\mathrm{N}_{\mathrm{B}}=$ Número de bobinas.

$\mathrm{N}_{\mathrm{P}}=$ Número de polos del rotor.

La relación es por cada 4 pares de polos 3 bobinas, aplicando la ecuación (5) se obtiene un número de 9 bobinas; con esta distribución se garantiza que se sincronicen 3 imanes con 3 bobinas al mismo tiempo [11], permitiendo que la fuerza electromotriz (fem) inducida se sume.

Para poder realizar la comparación entre generadores con diferentes conexiones de bobinas, se ha decidido, ensayar otro generador con 10 bobinas conectadas todas en paralelo; este arreglo es propuesto por [6] y garantiza que con este generador se puede cargar una batería.

\subsection{Número de espiras por bobina}

Las dimensiones de la bobina quedan determinadas por las medidas del imán ya que la bobina debe proporcionar una sección hueca para el paso del campo magnético, como se trata de bobinas con núcleo de aire con soporte se considera una inductancia de $1 \mathrm{mH}$ para este dimensionamiento.

$$
n=\sqrt{\frac{l L}{\mu A}}
$$

Donde:

$\mathrm{n}=$ Número de espiras.

$l=$ Longitud de la bobina $(0,013 \mathrm{~m})$.

$\mathrm{L}=$ Inductancia $(1 \mathrm{mH})$.

$\mu=$ Permeabilidad del núcleo $\left(4 \pi \times 10^{-7} \mathrm{Hm}^{-1}\right)$.

$\mathrm{A}=$ Área de la sección transversal $\left(1,2 \times 10^{-3} \mathrm{~m}^{2}\right)$.

Por cuestiones de espacio y empaquetamiento se ha utilizado para la construcción de las bobinas alambre AWG 14 con un diámetro de 1,63 mm [6].

En la Tabla 2 se recogen los parámetros necesarios para la construcción del estator.

Tabla 2. Parámetros estator

\begin{tabular}{lccc}
\hline & $\begin{array}{c}\text { Número de } \\
\text { polos (p) }\end{array}$ & $\begin{array}{c}\text { Número de } \\
\text { bobinas (Nb) }\end{array}$ & $\begin{array}{c}\text { Número de } \\
\text { espiras por } \\
\text { bobina (n) }\end{array}$ \\
\hline $\begin{array}{c}\text { Generadores } \\
\text { en serie } \\
\begin{array}{c}\text { Generadores } \\
\text { en paralelo }\end{array}\end{array}$ & 24 & 9 & 93 \\
\hline
\end{tabular}

\subsection{Distribución de bobinas}

El estator construido debe permitir el paso de los tornillos que unen los rotores, por lo que se ha dejado la parte central vacía y con forma circular. Para esto [6] recomienda el uso de una «isla» que no es más que una sección circular de madera como se muestra en la Figura 8. El diámetro de la isla (DIS) es de $13 \mathrm{~cm}$.

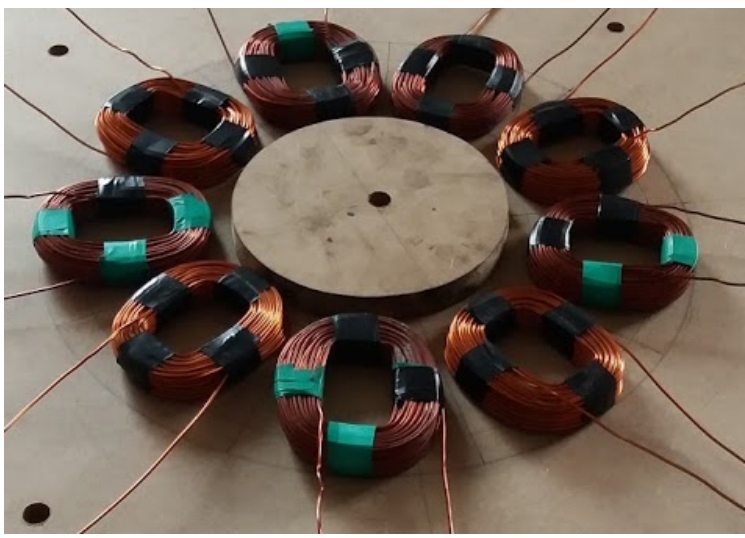

Figura 8. Ubicación de bobinas.

El estator terminado se puede ver en la Figura 9, el pie de las bobinas está ubicado a $1 \mathrm{~cm}$ del agujero. A partir de estas medidas se sitúan los imanes sobre discos de acero de forma que su perímetro coincida con los espacios internos de las bobinas. 


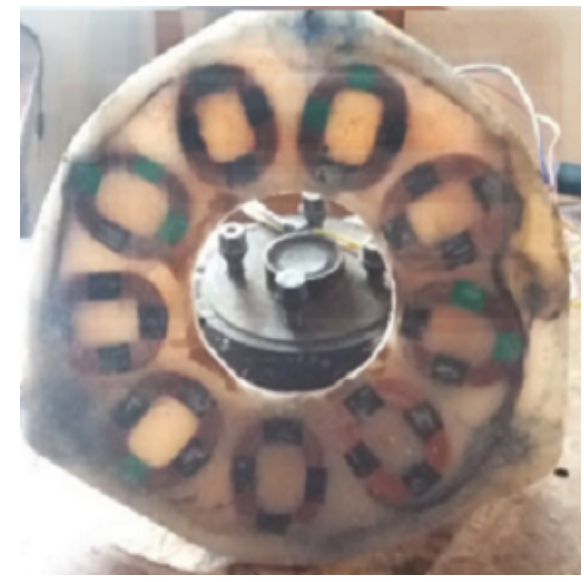

Figura 9. Estator

\subsection{Distribución de los imanes}

Se distribuyen los 12 imanes simétricamente en cada disco rotor, donde el diámetro de estos está en función del que tiene la isla, como se muestra en la Figura 10, la distancia al pie de las bobinas y el ancho de las mismas $(\mathrm{AB})$. Entonces el diámetro al pie de los imanes (DIM) expresado en centímetros es:

$$
D_{I M}=D_{I S}+2 \cdot A_{B}+2
$$

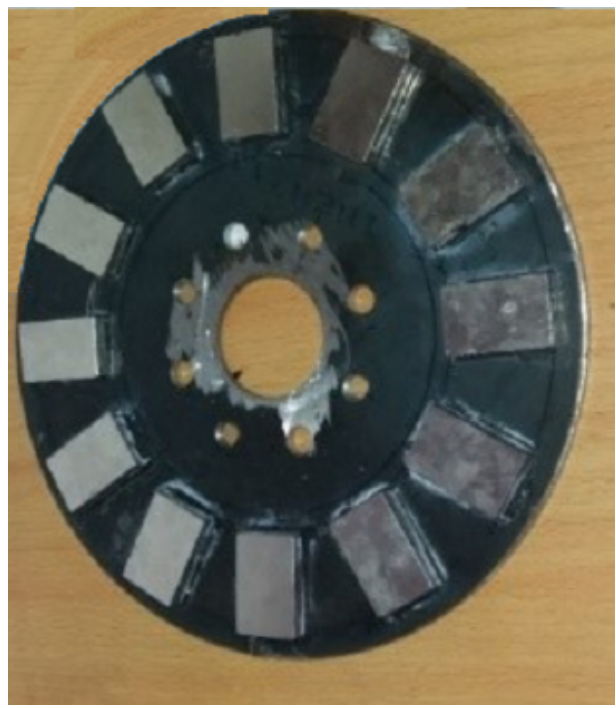

Figura 10. Ubicación de los imanes

\subsection{Determinación de las pérdidas eléctricas}

- Pérdidas por resistencia en las bobinas del generador en serie:

La resistencia medida en dos fases o 6 bobinas es $2,2 \Omega$ por lo tanto la resistencia por bobina es $0,37 \Omega$ para determinar la resistencia en el estator según [6] se considera que 2 fases siempre están trabajando y se deduce que la resistencia en el estator es:

$$
R_{E}=2 \cdot N_{B} \cdot R_{B}
$$

Donde:

$\mathrm{R}_{\mathrm{E}}=$ Resistencia en el estator.

$\mathrm{R}_{\mathrm{B}}=$ Resistencia por bobina.

Se tiene una resistencia en el estator de $2,22 \Omega$.

- Pérdidas por resistencia en las bobinas del generador en paralelo:

La resistencia por bobina es de $0,26 \Omega$ y por su configuración esa es la resistencia del estator debido a que solo una bobina funciona a la vez.

\section{- Pérdidas por resistencia en las bobinas:}

La resistencia que presentan las espiras de las bobinas impide que la corriente pueda fluir completamente libre, ya que todo material posee cierta resistencia eléctrica, de ahí se determina si los materiales son buenos o malos conductores, estas se evalúan mediante la ecuación:

$$
P_{B}=I^{2} \cdot R_{E}
$$

Donde:

$\mathrm{P}_{\mathrm{B}}=$ Potencia de pérdida en las bobinas.

$\mathrm{I}=$ Intensidad de corriente que circula en los bobinados.

\section{- Pérdidas en el rectificador:}

Luego de la recolección de corriente en los extremos de las bobinas lo siguiente es el proceso de rectificación, de alterna a directa. El método de rectificación escogido para esta aplicación es de onda completa, con puentes rectificadores. Para el generador en serie se utilizaron tres puentes y para el generador en paralelo cinco. La intención es aprovechar al máximo la corriente alterna generada. Cada fase se distribuye para cuatro diodos lo que reduce el calentamiento y, por lo tanto, su desgaste. La única desventaja de este arreglo es la caída de voltaje de 1,4 voltios para los dos generadores.

$$
P_{R}=I \cdot V
$$

Finalmente, las pérdidas eléctricas totales $\left(\mathrm{P}_{\mathrm{E}}\right)$ son:

$$
\begin{gathered}
P_{E}=P_{B}+P_{R} \\
P_{E}=I^{2} \cdot R_{E}+I \cdot V
\end{gathered}
$$

Mientras mayores sean las pérdidas menor será la eficiencia, pero para poder determinar esto se deben analizar las tendencias de generación. 


\subsection{Determinación de eficiencias}

La potencia de salida $\left(\mathrm{P}_{\mathrm{S}}\right)$ es obtenida a partir del voltaje y amperaje medidos, la eficiencia ( $\eta$ ) está determinada por la potencia de salida neta y las pérdidas, el generador debe proporcionar el valor de potencia neta más las pérdidas, por lo tanto:

$$
\eta=\frac{P_{S}}{P_{S}+P_{E}}
$$

\section{Resultados y discusión}

Los datos de tensión e intensidad de corriente fueron obtenidos haciendo funcionar cada uno de los generadores a diferentes valores de velocidad angular (en rpm), haciendo girar los rotores en el mandril de un torno como se puede observar en la Figura 11. Para provocar carga en el generador se utilizó un reóstato con distintos valores de resistencia conectado a la salida de este.

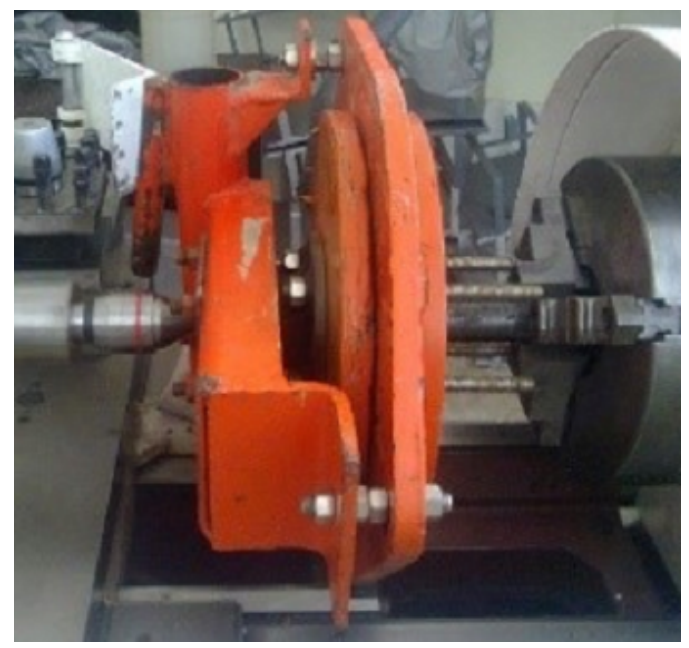

Figura 11. Montaje del generador en el torno.

Mediante este arreglo se obtuvieron los datos de voltaje y amperaje al mismo tiempo con la ayuda de dos multímetros conectados como en la Figura 12.

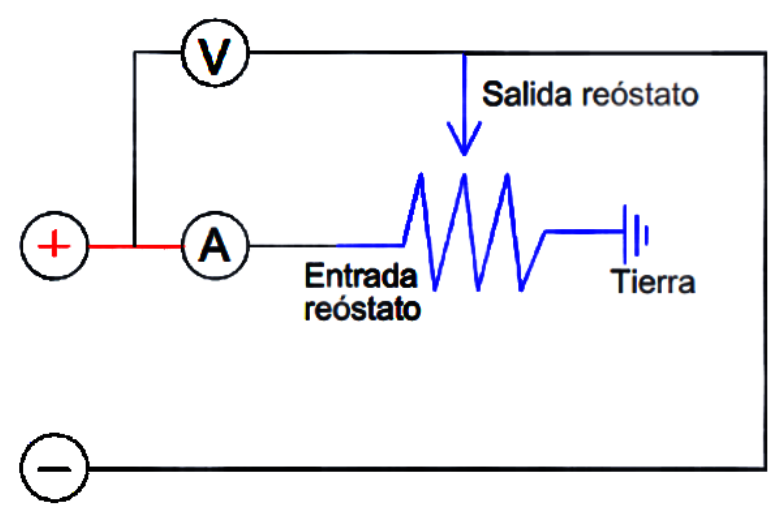

Figura 12. Esquema de conexión.
Con los datos obtenidos se realizan gráficas tomando en cuenta que todos los valores están en función de la velocidad angular ya que de esta dependen principalmente las variaciones de generación.

En las Figuras 13 a 15 se presentan las gráficas con los datos del generador de bobinas en paralelo:

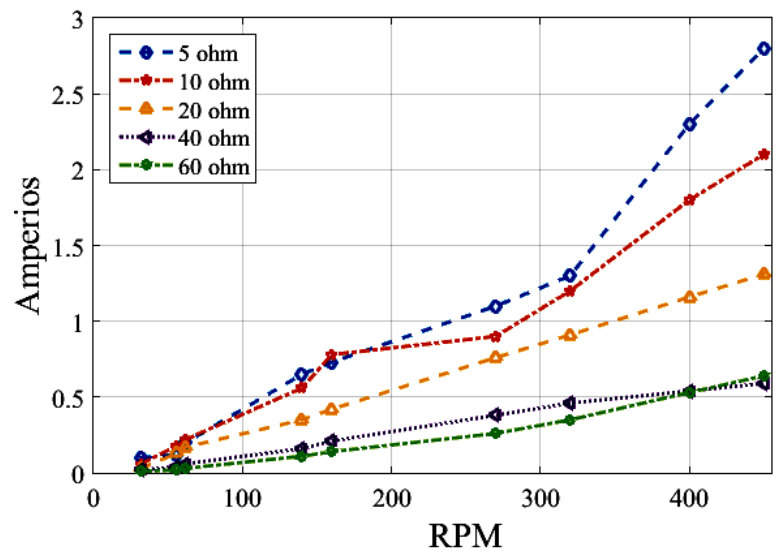

Figura 13. Intensidad de corriente a distintos valores de resistencia.

La variación de la intensidad es proporcional a la velocidad angular como se muestra en la Figura 13, observándose que cuanta más alta sea la resistencia de la carga y aumente la velocidad angular, disminuye el amperaje.

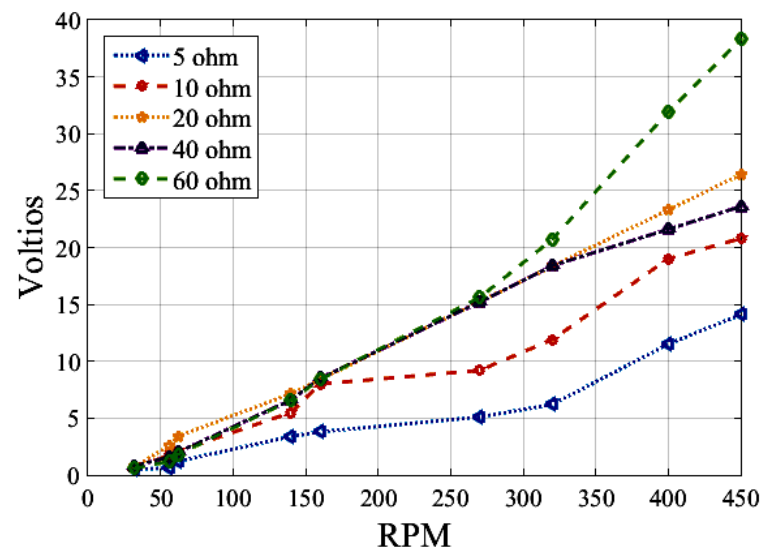

Figura 14. Fuerza electromotriz a distintos valores de resistencia.

Como la tensión es proporcional a la corriente de acuerdo con la ley de Ohm, se observa en la Figura 14 un incremento de voltaje a medida que aumenta el valor de la resistencia, siendo más significativo a altas velocidades angulares.

En la Figura 15 se muestra el comportamiento de la potencia del generador frente a su velocidad angular. Hasta los $250 \mathrm{rpm}$ no hay un aumento importante de potencia. Cuando aumenta la velocidad de los rotores la potencia se incrementa siendo más evidente ese aumento a baja resistencia. 


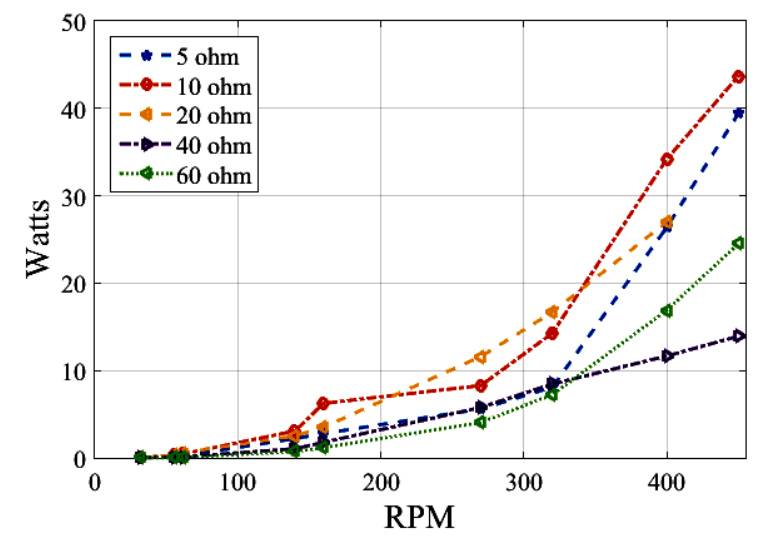

Figura 15. Potencias a distintos valores de resistencia.

En las Figuras 16 a 18 se presentan las gráficas con los datos del generador de bobinas en serie.

El comportamiento del amperaje es creciente en todos los casos como se muestra en la Figura 16 y se debe destacar que mientras menor sea la resistencia mayor es el amperaje.

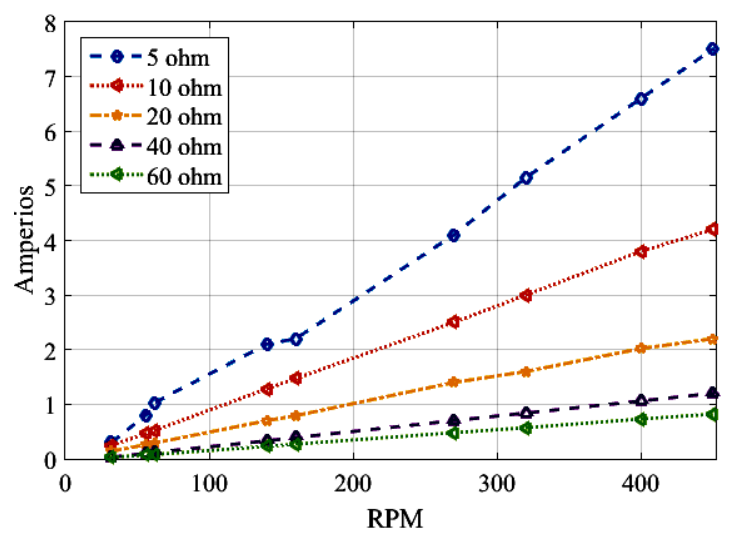

Figura 16. Amperajes a distintos valores de resistencia.

En la Figura 17 se aprecia que hay variaciones importantes en los valores de voltaje. Si se toma crecimientos del $100 \%$, tanto en resistencia como en velocidad angular, el voltaje tiene un crecimiento del $120 \%$ a una misma carga.

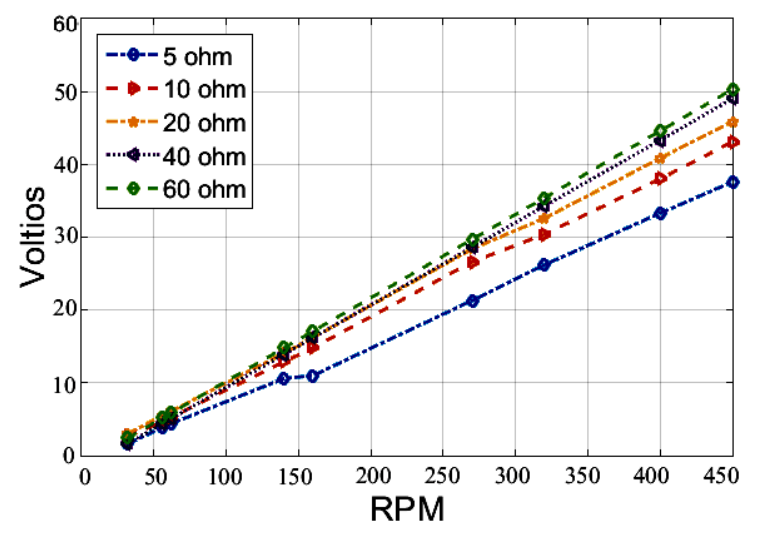

Figura 17. Voltajes a distintos valores de resistencia.
Esta es una característica muy favorable para este generador ya que a pesar de que su amperaje cambie notablemente como se vio en la Figura 16, el comportamiento del voltaje es lineal.

$\mathrm{El}$ incremento de la resistencia impide el libre paso de corriente como se puede apreciar en la Figura 18.

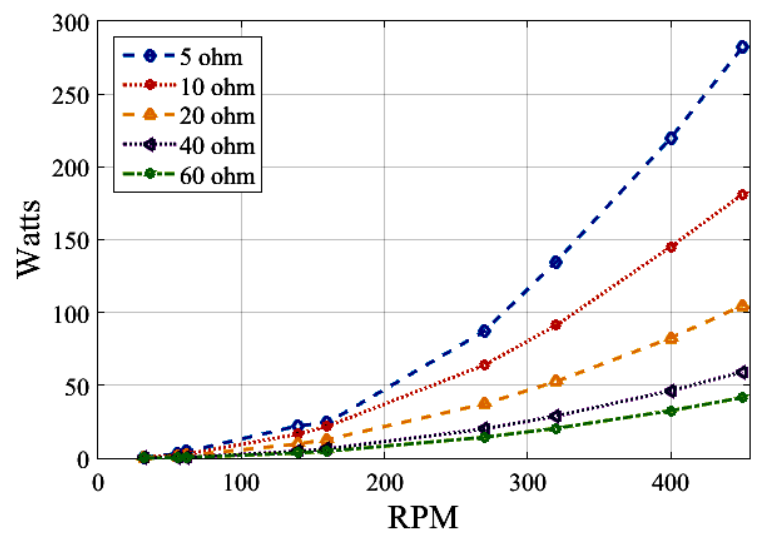

Figura 18. Potencias a distintos valores de resistencia.

Esto ocasiona que la potencia sea menor a alta carga a pesar del aumento de la velocidad angular en el generador.

En las Figuras 19 a 22 se comparan las características teóricas con las experimentales de los generadores en serie y paralelo. El valor escogido para la resistencia es de $5 \Omega$, que genera el amperaje o corriente suficiente, así como también, el voltaje a velocidades angulares comunes en el uso de microgeneradores eólicos. Además, se debe vencer la resistencia provocada por un regulador de carga, el cableado y las baterías a cargar.

A pesar de que el generador de bobinas en paralelo posee una bobina más que el generador en serie, se puede notar claramente en la Figura 19 la diferencia de voltajes.

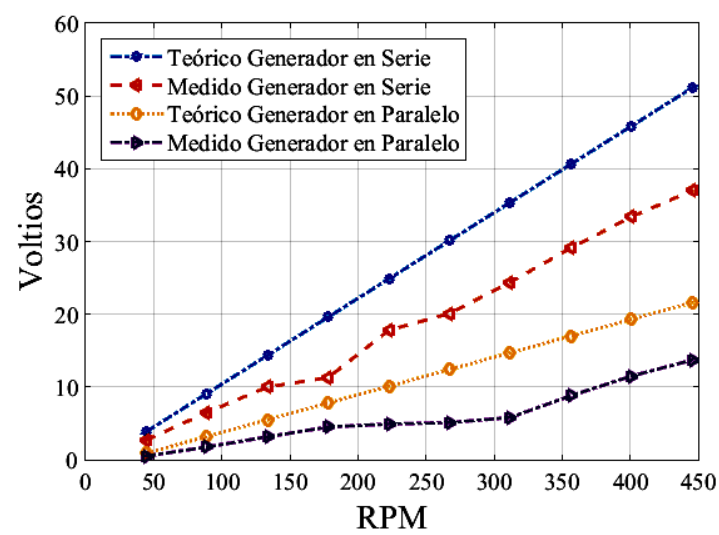

Figura 19. Comparaciones de voltajes teórico y medido.

Esto se debe a que en el generador en serie las tres bobinas actúan como una sola por su conexión, con la diferencia de que son tres pares de imanes los que actúan a la vez. Esto ocasiona que el voltaje sea 
mucho mayor que en el caso del generador de bobinas en paralelo, en el que solo actúa una bobina a la vez con un par de imanes.

Como se puede observar en la Figura 20 el generador en serie supera al generador en paralelo al proporcionar mayor amperaje. Esto permite cargar rápidamente la batería para el almacenamiento de la energía o se puede incrementar su número.

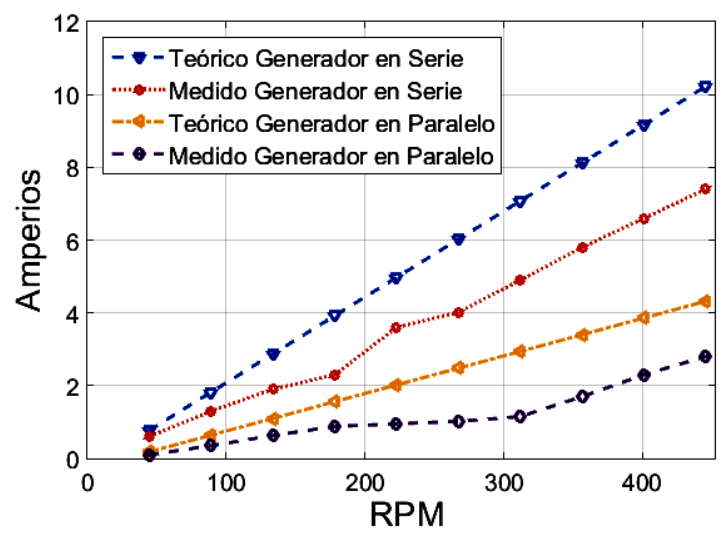

Figura 20. Comparaciones de amperajes teórico y medido.

Las potencias determinadas en la Figura 21 no son más que el reflejo de los valores de voltaje y amperaje, por lo tanto, son directamente proporcionales.

En el caso de la potencia, los valores medidos tienden a comportarse de manera cuadrática.

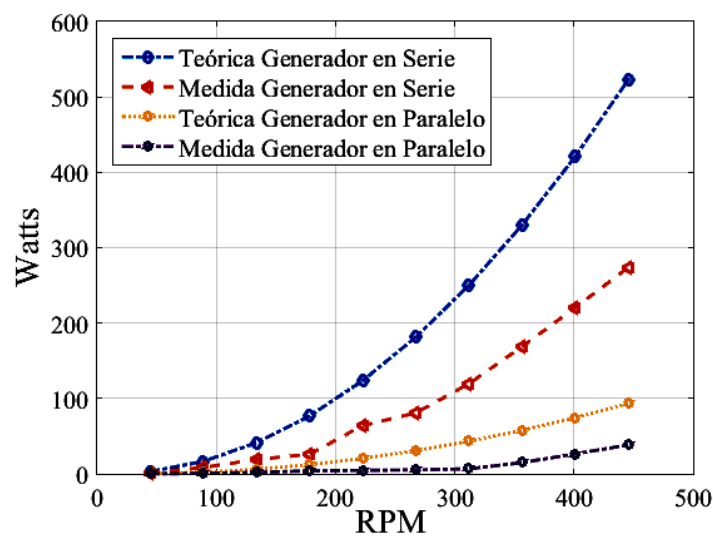

Figura 21. Comparaciones de potencias teórica y medida.

El amperaje y el voltaje obtenidos en ambas pruebas del generador de bobinas en serie son mayores e implica que su potencia también lo sea.

En cuanto a la eficiencia de los generadores, la Figura 22 muestra que a pesar de que el generador en paralelo sea superado en voltaje y amperaje por el generador en serie, es más eficiente.

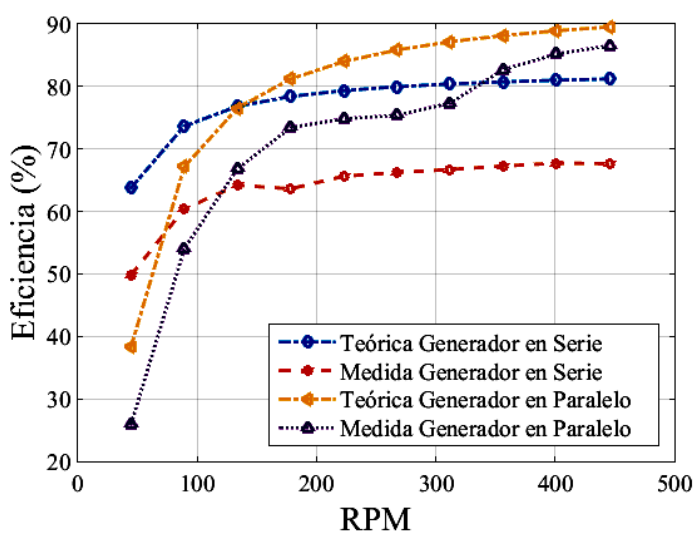

Figura 22. Comparaciones de eficiencia teórica y medida.

Este hecho es razonable ya que en el generador en serie las resistencias por fase se suman, provocando más pérdidas; en el generador en paralelo solo se tiene una resistencia por fase.

Estas cualidades deben ser consideradas para su selección tomando en cuenta el requerimiento energético. Si se necesita voltajes y amperajes elevados y una eficiencia razonable, se ha de seleccionar el generador en serie; pero si lo que se busca es mayor estabilidad de voltaje y amperaje a bajas revoluciones con mayor eficiencia el generador en paralelo es el indicado.

\section{Conclusiones}

Los principales parámetros para el dimensionamiento de un generador de flujo axial son la selección de imanes, determinación de la densidad de campo, número de polos, número de bobinas, número de espiras por bobina, calibre del cable para las bobinas y distribución simétrica de imanes y bobinas.

Las dimensiones de los discos para los rotores y las medidas del estator están determinadas por la disposición de las bobinas, cuanto más grandes sean estas y mayor su número, más grandes serán los discos y por lo tanto el estator.

El estudio mostró que la mejor configuración para la conexión de bobinas es en serie por el mejor aprovechamiento de la distribución de imanes y la cualidad de sumar las $f_{\text {em }}$ de cada grupo de bobinas. Sin embargo, el generador en serie presenta mayores pérdidas, lo que indica que tendrá más problemas por calentamiento que el generador en paralelo.

Los generadores de flujo axial proporcionan voltajes y amperajes muy buenos a bajas revoluciones. Esto destaca los beneficios que tienen estos generadores para aplicaciones en energía eólica a pesar de que se tenga un potencial eólico bajo o limitado.

Si se compara los resultados teóricos con los experimentales, los generadores de flujo axial son adecuados para generación de electricidad a pequeña escala. Si el generador trifásico es probado en un lugar en que 
la velocidad media de viento esté comprendida entre 4 a $8 \mathrm{~m} / \mathrm{s}$, con velocidades de giro de rotor de 220 a $450 \mathrm{rpm}$; el amperaje alcanzado va de 3 a 7 amperios; además, si se considera que en el día se tienen de 4 a 8 horas con esta velocidad de viento, se tiene de 18 a 24 amperios-hora en el día aproximadamente, lo que representa una cuarta parte del amperaje total de una batería común de $100 \mathrm{Ah}$.

\section{Referencias}

[1] I. Arraña, E. Marino, P. Bertinat, J. Salerno, J. Chemes, M. Barone, and J. Sáenz, "Estado del arte en el desarrollo de pequeños generadores eólicos," Avances en Energías Renovables y Medio Ambiente, vol. 16, pp. 41-48, 2012. [Online]. Available: https://goo.gl/kQEezZ

[2] WWEA, "Reporte anual de la energía eólica en el mundo 2010," in 10a Conferencia Mundial sobre Energía Eólica y Exhibición de Energías Renovables, 2011. [Online]. Available: https://goo.gl/z7wYsP

[3] J. R. Bumby and R. Martin, "Axial-flux permanent-magnet air-cored generator for smallscale wind turbines," IEE Proceedings - Electric Power Applications, vol. 152, no. 5, pp. 1065-1075, 2005. [Online]. Available: https://doi.org/10.1049/ip-epa:20050094

[4] R. Wallace and A. Rodríguez, "Diseño de un generador de flujo axial con imanes permanentes para aplicaciones eólicas," Revista Facultad de Ingeniería, pp. 3-12, 2000. [Online]. Available: https://goo.gl/WwuQfq

[5] A. Carvajal and L. Muñoz, "Ecoductos energéticos: Explotación de la energía cinética del gas para generación de energía eléctrica." Tesis de Grado, Universidad Nacional Abierta y a
Distancia, Colombia, 2012. [Online]. Available: https://goo.gl/bw6JpJ

[6] H. Piggott, "Manual para la autoconstrucción de generadores eólicos." Bajatec manuales, pp. 24-60, 2009.

[7] R. Muñoz, "Generalidades sobre imanes permanentes y su caracterización." Instituto Nacional de Tecnología Industrial, 2008. [Online]. Available: https://goo.gl/U4wrrX

[8] L. Herrera, A. Alarcón, and E. Rivas-Trujillo, "Diseño de un generador de flujo axial usando el método de elementos finitos," Redes de Ingeniería, vol. 4, no. 2, pp. 6-15, 2013. [Online]. Available: https://goo.gl/wD7ywj

[9] C. Brad, I. Vadan, and I. Berinde, "Design and analysis of an axial magnetic flux wind generator," in 2017 International Conference on Modern Power Systems (MPS), June 2017, pp. 1-7. [Online]. Available: https://doi.org/10.1109/MPS.2017.7974377

[10] Supermagnete. (2016) Bloques magnéticos. [Online]. Available: https://goo.gl/S3N7E5

[11] P. Wannakarn, T. Tanmaneeprasert, N. Rugthaicharoencheep, and S. Nedphograw, "Design and construction of axial flux permanent magnet generator for wind turbine generated dc voltage at rated power $1500 \mathrm{w}$," in 2011 4th International Conference on Electric Utility Deregulation and Restructuring and Power Technologies (DRPT), July 2011, pp. 763-766. [Online]. Available: https://doi.org/10.1109/DRPT.2011.5993994

[12] J. Murillo, "Diseño de un alternador de flujo axial con imanes permanentes," Escuela Universitaria de Ingeniería Técnica Industrial, 2012. [Online]. Available: https://goo.gl/pbqSEz 


\title{
ANÁLISIS ENERGÉTICO, EXERGÉTICO Y ECONÓMICO DE UN SISTEMA DE COGENERACIÓN: CASO PARA UNA PLANTA AZUCARERA DE SAN PABLO
}

\section{ENERGETIC, EXERGETIC AND ECONOMIC ANALYSIS OF A COGENERATION SYSTEM: CASE FOR A SUGARCANE PLANT OF SÃO PAUlO}

\author{
Omar R. Llerena P. ${ }^{1}$
}

\section{Resumen}

Los sistemas de cogeneración, debido a las numerosas ventajas que presentan, se han convertido en una tecnología bastante utilizada para la generación de energía. En estos procesos, la eficiencia es relativamente alta y las emisiones de gases de efecto invernadero son bajas. En este trabajo se realiza un estudio técnico y económico de un sistema de cogeneración para una planta azucarera en San Pablo. La primera y segunda ley de la termodinámica son aplicadas para el análisis técnico. Los costos de producción de la energía eléctrica y vapor producido son determinados en el análisis económico. En el dimensionamiento se analizan cuatro turbinas de gas que están en paridad térmica con el proceso. Los resultados muestran que la planta tiene una capacidad para producir $148 \mathrm{MW}$ de electricidad y $147 \mathrm{MW}$ de vapor. Por un lado el análisis energético revela que la eficiencia de la planta es de $67 \%$, mientras que el análisis exergético muestra que esta eficiencia es de $56 \%$. Los resultados del análisis económico expresan que los precios de la electricidad y vapor producido son de 0,105 y 0,068 US $\$ / \mathrm{kWh}$, respectivamente.

Palabras clave: Cogeneración, análisis económico, análisis energético, análisis exergético.

\section{Abstract}

Due to many advantages that cogeneration systems present, they have become a widely used technology for energy generation. In these processes, the efficiency is relatively high and the emissions of greenhouse gases are low. In this paper a technical and economic study of a cogeneration system for a sugar plant in São Paulo is carried out. For this propose the 1st and 2nd laws of thermodynamics are applied for the technical analysis. The cost of the electrical energy and steam produced are determined in the economic analysis. In the sizing of the plant, four gas turbines are analyzed that are in thermal parity with the process. The results show that the plant has a production capacity of $148 \mathrm{MW}$ of electricity and $147 \mathrm{MW}$ of steam. On the one hand the energy analysis reveals that the efficiency of the plant is $67 \%$, while the exergetic analysis shows that this efficiency is $56 \%$. The results of the economic analysis indicate that the prices of electricity and steam produced are 0.105 and 0.068 US $\$ / \mathrm{kWh}$, respectively.

Keywords: Cogeneration, economic analysis, energetic analysis, exergetic analysis.

\footnotetext{
${ }^{1}$ Grupo de Optimización de Sistemas Energéticos (GOSE), Universidad Estatal de San Pablo - Brasil. Autor para correspondencia ollerenap@yahoo.com. (1) http://orcid.org/0000-0003-2115-4036
} 


\section{Introducción}

La cogeneración es el proceso de producción de energía combinada a partir de una única fuente de combustible. Por ejemplo, la producción de energía térmica y eléctrica a partir del gas natural [1]. Este término no es nuevo; las plantas industriales han utilizado el concepto de cogeneración a principios de 1880 cuando el vapor era la principal fuente de energía. Sin embargo, en la actualidad este tipo de procesos todavía desempeñan un papel importante en la producción de energía, pues, sus bajos costos de inversión, operación y mantenimiento, así como su mayor eficiencia y menor impacto ambiental los hacen más atractivos [2-4].

El ciclo combinado (CC) es uno de los sistemas de cogeneración más común. Este ciclo trabaja en dos etapas: la primera funciona a alta temperatura (turbina de gas) y la segunda etapa del ciclo a una temperatura menor (utiliza la energía térmica de los gases de escape de la turbina de gas para producir vapor) [5]. Los $\mathrm{CC}$ han recibido mucho reconocimiento en las últimas décadas y por lo tanto varias plantas se han instalado $\mathrm{y}$ algunas unidades existentes han sido reactivadas [6]. Este hecho hace que los análisis técnicos y económicos sean usados con mayor frecuencia con el objetivo de optimizar el rendimiento de las plantas.

Por otro lado, la primera y segunda ley de la termodinámica son herramientas importantes para mejorar la eficiencia de este tipo de procesos y disminuir la irreversibilidad. De la misma manera, el análisis económico es considerado también como una poderosa herramienta para estudiar y optimizar los sistemas energéticos. Este análisis es aplicable en las decisiones de inversión [7]. Según Silveira y Tuna [8], el objetivo del análisis económico es evaluar el costo de la energía producida por el sistema de cogeneración (electricidad y vapor). En estos contextos, varios estudios se han reportado en la literatura especializada. Kordlar y Mahmoudi [9] presentan un análisis exergo económico y la optimización de un nuevo sistema de cogeneración que produce energía y refrigeración. Por otro lado, Gambini y Vellini [10], así como Gvozdenac et al. [11] muestran un análisis en sistemas de cogeneración de alta eficiencia.

Kanbur et al. [12] presentan una evaluación termodinámica de un sistema de microcogeneración. Los resultados muestran que las eficiencias exergéticas y energéticas son entre dos y tres veces mayores que el caso de generación de energía convencional. Sun [13] en su trabajo determinó la eficiencia energética y analizó la viabilidad económica de un sistema de cogeneración impulsado por un motor. En este caso el sistema de cogeneración proporcionaba electricidad y refrigeración/calefacción para edificios. Aquí, los resultados muestran que el ahorro de energía primaria del sistema de cogeneración es superior al $37 \%$ en comparación con el sistema convencional.
Por su lado, Abusoglu y Kanoglu [14] aplicaron la primera y segunda ley de la termodinámica a sistemas de cogeneración con motores a diésel. En este estudio los resultados muestran que la eficiencia térmica global de la planta se encuentra en un 44,2 \% y la eficiencia exergética es del 40,7\%.

Como se muestra en la literatura especializada, existen varios estudios para la evaluación técnica y económica de sistemas de cogeneración hasta la fecha. El presente trabajo difiere de estos artículos estudiados en la literatura; pues es realizado un análisis energético, exergético y económico a un sistema de cogeneración en particular, con miras a ser utilizado en una planta azucarera de San Pablo. La demanda térmica del proceso (parámetro principal para el dimensionamiento), el tipo de turbina de gas y la metodología utilizada para los análisis hacen de este trabajo un artículo inédito.

Este trabajo está estructurado de la siguiente manera: La Sección 2 presenta el caso estudiado. La metodología adoptada para el análisis energético, exergético y evaluación económica se describe paso a paso en la Sección 3. Los resultados y discusiones son mostrados en la Sección 4 y finalmente, las conclusiones son presentadas en la Sección 5.

\section{Descripción del sistema}

\subsection{Descripción de la planta azucarera}

En la industria azucarera el principal sistema de cogeneración emplea turbinas de vapor en tres configuraciones fundamentales: turbinas de contrapresión, combinación de turbinas de contrapresión con otras de condensación y turbinas de extracción-condensación [15]. En este caso, la planta a ser analizada en el presente trabajo cuenta con dos de las configuraciones antes mencionadas (turbinas a contrapresión y extraccióncondensación) y se trata de una planta existente en San Pablo - Brasil (ver Figura 1).

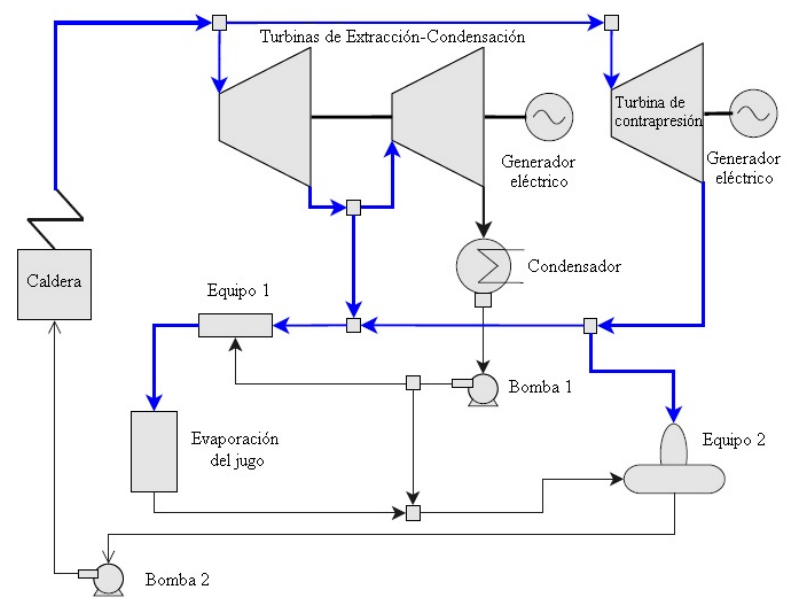

Figura 1. Planta azucarera existente en San Pablo, Brasil. 
La caldera convencional de esta planta tiene una capacidad de producir $160 \mathrm{t} / \mathrm{h}$ de vapor a 68,6 bar y $530{ }^{\circ} \mathrm{C} .125 \mathrm{t} / \mathrm{h}$ de este vapor es consumido por la turbina de extracción-condensación que está acoplada a un generador de $32 \mathrm{MW}$. En esta sección es realizada una extracción de $97 \mathrm{t} / \mathrm{h}$ de vapor a una presión de 2,45 bar para el proceso de evaporación del jugo de caña. El restante del vapor $(35 \mathrm{t} / \mathrm{h})$ es direccionado a la turbina de contrapresión la cual está acoplada a un generador de $12 \mathrm{MW}$.

Adicionalmente, la planta analizada en este trabajo tiene la capacidad de procesar 1500000 toneladas de caña que corresponden a 240 días de zafra. Muele $286 \mathrm{t} / \mathrm{h}$ de caña y genera $75,2 \mathrm{t} / \mathrm{h}$ de bagazo. Este último es utilizado como combustible en calderas convencionales para generar el vapor necesario para la planta [15].

\subsection{Descripción del sistema propuesto}

El sistema de cogeneración propuesto tiene que satisfacer las mismas demandas térmicas (160 t/h de vapor) y generar por lo menos 4 veces más energía eléctrica. Para conseguir este objetivo se analiza en este trabajo la posibilidad de cambiar la caldera convencional que produce el vapor (ver Figura 1) por una turbina de gas y una caldera de recuperación como se muestra en la Figura 2.

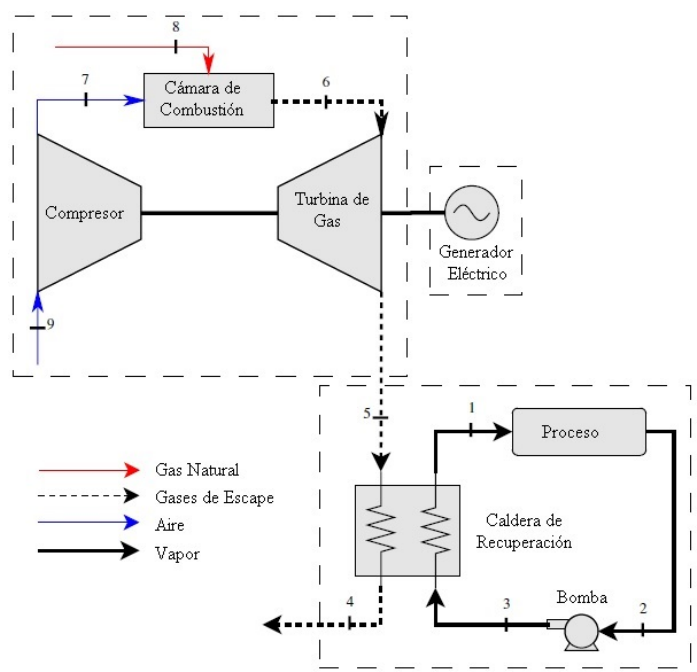

Figura 2. Esquema del sistema de cogeneración propuesto.

Como observado en la Figura 2, el sistema está compuesto por una turbina de gas, una cámara de combustión (CC), un compresor, una caldera de recuperación $(\mathrm{CR})$, un generador, una bomba y el proceso. Este último engloba casi todos los equipos de la planta de la Figura 1, el único elemento que queda fuera es la caldera convencional.

Se optó por un sistema de ciclo combinado usando gas natural con el objetivo de conocer la viabilidad de instalar este proceso específicamente en esta industria azucarera. En caso de ser factible la instalación del $\mathrm{CC}$, en posteriores estudios se realizará el análisis de la gasificación de los 75,2 t/h de bagazo de caña generado por la planta para producir gas de síntesis y sustituir el uso del gas natural.

\section{Materiales y métodos}

\subsection{Selección de la turbina de gas}

La primera etapa en el diseño de este proceso de cogeneración es la selección de la turbina de gas. Esta turbina es seleccionada en paridad a la demanda térmica del proceso. Así, la selección de la turbina de gas se realiza en función de la temperatura y el flujo de los gases de escape. Este último es determinado por la Ecuación 1 [16]:

$$
\dot{m}_{5}=\frac{\dot{m}_{1}\left(h_{1}-h_{3}\right)}{\eta_{C R} C p_{G}\left(T_{5}-T_{4}\right)}
$$

Donde:

$\dot{\mathrm{m}}_{5}=$ flujo de los gases de escape $(\mathrm{kg} / \mathrm{s})$

$\dot{\mathrm{m}}_{1}=$ flujo de vapor en la $\mathrm{CR}(\mathrm{kg} / \mathrm{s})$

$\mathrm{h}_{3}=$ entalpía de vapor en la $\mathrm{CR}(\mathrm{kJ} / \mathrm{kg})$

$\mathrm{h}_{1}=$ entalpía del agua $(\mathrm{kJ} / \mathrm{kg})$

$\eta_{\mathrm{CR}}=$ rendimiento de la $\mathrm{CR}(-)$

$\mathrm{Cp}_{\mathrm{G}}=$ calor específico a presión constante de los gases de escape $(\mathrm{kJ} / \mathrm{kg} \cdot \mathrm{K})$

$\mathrm{T}_{5}=$ temperatura de gases de escape en la $\mathrm{CR}\left({ }^{\circ} \mathrm{C}\right)$

$\mathrm{T}_{4}=$ temperatura de gases de escape a la salida de la $\mathrm{CR}\left({ }^{\circ} \mathrm{C}\right)$

\subsubsection{Cálculo del $\mathrm{Cp}_{\mathrm{G}}$}

Para aplicar la Ecuación 1 es necesario el cálculo del $\mathrm{Cp}_{\mathrm{G}}$, este es determinado con la Ecuación 2 [17]:

$$
C p_{G}=\frac{\sum C p_{i} \cdot n_{i}}{\sum \dot{m}_{i}}
$$

Para el cálculo del $\mathrm{Cp}_{\mathrm{i}}$ se tiene la Ecuación 3 .

$$
C p_{i}=a+b T+c T^{2}+d T^{3}
$$

Donde:

$\mathrm{Cp}_{\mathrm{G}}=$ calor específico a presión constante de los gases de escape $(\mathrm{kJ} / \mathrm{kg} \cdot \mathrm{K})$

$\mathrm{Cp}_{\mathrm{i}}=$ calor específico a presión constante del componente i de los gases de escape $(\mathrm{kJ} / \mathrm{kg} \cdot \mathrm{K})$

$\dot{\mathrm{m}}_{\mathrm{i}}=$ flujo de los gases de escape $(\mathrm{kg} / \mathrm{s})$

$\dot{\mathrm{n}}_{\mathrm{i}}=$ flujo molar del componente $\mathrm{i}$ de los gases de escape $(\mathrm{kmol} / \mathrm{s})$

Los parámetros a, b, c, d son obtenidos en Çengel y Boles [17] y son los factores para calcular el calor específico a presión constante de un gas ideal. 


\subsubsection{Cálculo de la masa de los gases de escape}

Para realizar este cálculo se define primeramente la composición del gas natural (Tabla 1) que será utilizado.

Tabla 1. Composición química del gas natural [18]

\begin{tabular}{cccc}
\hline Compuesto & Fórmula & \% Vol & \% Molar \\
\hline Metano & $\mathrm{CH}_{4}$ & 95 & 1 \\
Etano & $\mathrm{C}_{2} \mathrm{H}_{6}$ & 3,2 & 0,0337 \\
Propano & $\mathrm{C}_{3} \mathrm{H}_{8}$ & 0,2 & 0,0021 \\
Butano & $\mathrm{C}_{4} \mathrm{H}_{10}$ & 0,03 & 0,0003 \\
Nitrógeno & $\mathrm{N}_{2}$ & 1 & 0,0105 \\
Dióxido de & $\mathrm{CO}_{2}$ & 0,55 & 0,0058 \\
carbono & $\mathrm{O}_{2}$ & 0,02 & 0,0002 \\
Oxígeno & \\
\hline
\end{tabular}

Con la composición del gas natural se establece la Ecuación 4:

$$
\begin{array}{r}
\mathrm{CH}_{4}+0,0337 \mathrm{C}_{2} \mathrm{H}_{6}+0,0021 \mathrm{C}_{3} \mathrm{H}_{5}+0,0003 \ldots \\
+0,0105 \mathrm{~N}+0,0058 \mathrm{CO}_{2}+0,0002 \mathrm{O}_{2} \ldots \\
+(1+x) a_{t h}\left(\mathrm{O}_{2}+3,76 \mathrm{~N}_{2}\right) \rightarrow a C \mathrm{O}_{2}+b \mathrm{H}_{2} \mathrm{O} \ldots \\
+x a_{t h} \mathrm{O}_{2}+d N_{2}
\end{array}
$$

Después de la solución de la Ecuación 4 se procede al cálculo de la masa del combustible y masa del aire.

Para la masa del combustible se tiene la Ecuación 5:

$$
\begin{array}{r}
\dot{m}_{\text {comb }}=m_{\text {molar }} \mathrm{CH}_{4}+0,0337 m_{\text {molar }} C_{2} H_{6} \ldots \\
+0,0021 m_{\text {molar }} C_{3} H_{5}+0,0003 m_{\text {molar }} C_{4} H_{10} \ldots \\
+0,0105 m_{\text {molar }} N_{2}+0,0058 m_{\text {molar }} C_{2} \ldots \\
+0,0002 m_{\text {molar }} O_{2}
\end{array}
$$

Para la masa del aire se tiene la Ecuación 6:

$$
\dot{m}_{a r}=(1+x) a_{t h}\left(m_{\text {molar }} O_{2}+3,76 m_{\text {molar }} N_{2}\right)
$$

La relación aire combustible es calculada con la Ecuación 7:

$$
A C=\frac{\dot{m}_{a r}}{\dot{m}_{c o m b}}
$$

Donde:

$\dot{\mathrm{m}}_{\mathrm{ar}}=$ flujo de aire $(\mathrm{kg} / \mathrm{s})$

$\dot{\mathrm{m}}_{\text {comb }}=$ flujo de combustible $(\mathrm{kg} / \mathrm{s})$

Así la ecuación compensada de la reacción es obtenida y representada en la 8:

$$
\begin{array}{r}
\mathrm{CH}_{4}+0,0337 \mathrm{C}_{2} \mathrm{H}_{6}+0,0021 \mathrm{C}_{3} \mathrm{H}_{5}+0,0003 \ldots \\
\mathrm{C}_{4} \mathrm{H}_{10}+0,0105 \mathrm{~N}+0,0058 \mathrm{CO}_{2}+0,0002 \mathrm{O}_{2} \ldots \\
+(1+x) a_{t h}\left(\mathrm{O}_{2}+3,76 \mathrm{~N}_{2}\right) \rightarrow 1,0807 \mathrm{CO}_{2} \ldots \\
+2,1111 \mathrm{H}_{2} \mathrm{O}+4,3220 \mathrm{O}_{2}+24,2710 \mathrm{~N}_{2}
\end{array}
$$

A partir de la Ecuación 8 se obtiene la masa de los gases de escape, la cual es calculada con la Ecuación 9:

$$
\begin{array}{r}
\dot{m}_{\text {escape }}=1,0807 m_{\text {molar }} \mathrm{CO}_{2}+2,1111 m_{\text {molar }} \ldots \\
\mathrm{H}_{2} \mathrm{O}+4,3220 m_{\text {molar }} \mathrm{O}_{2}+24,2710 m_{\text {molar }} \mathrm{N}_{2}
\end{array}
$$

Ahora, las ecuaciones 2 y 3 pueden ser aplicadas para la obtención del $\mathrm{Cp}_{\mathrm{G}}$. La Figura 3 muestra el $\mathrm{Cp}_{\mathrm{G}}$ obtenido para un rango de temperaturas.

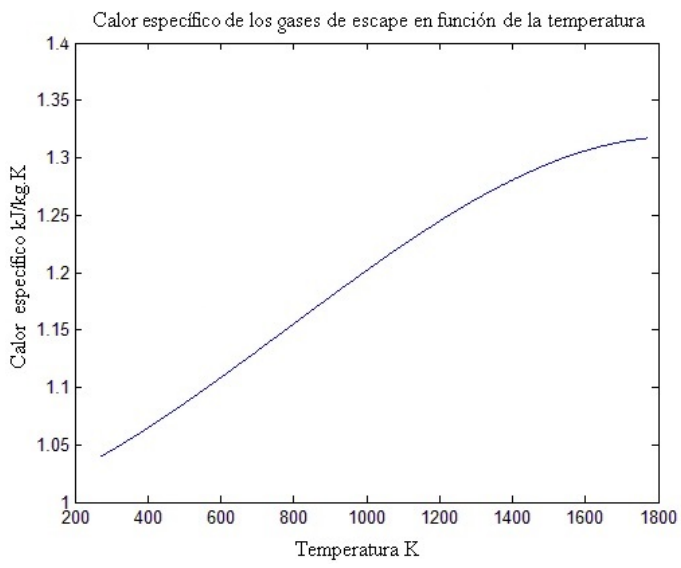

Figura 3. Calor específico de los gases de escape en función de la temperatura.

Finalmente, con el $\mathrm{Cp}_{\mathrm{G}}$ la Ecuación 1 puede ser aplicada. La Tabla 2 muestra las turbinas seleccionadas y la Figura 4 muestra la ubicación de cada turbina según el flujo de los gases de escape.

Tabla 2. Turbinas seleccionadas [19]

\begin{tabular}{ccccc}
\hline Código & $\begin{array}{c}\text { Potencia } \\
(\mathbf{M W})\end{array}$ & Eficiencia & $\begin{array}{c}\text { Cases } \\
\text { escape } \\
(\mathbf{k g} / \mathbf{s})\end{array}$ & $\begin{array}{c}\text { Temperatura } \\
\text { gases } \\
\left({ }^{\circ} \mathbf{C}\right)\end{array}$ \\
\hline V94.2 & 157 & 34,4 & 514,54 & 540 \\
PG7241 & 171 & 36,5 & 455,9 & 599,44 \\
SGT6 & 208 & 38,1 & 508,18 & 577,77 \\
7EA & 184 & 38,1 & 451,36 & 604,44 \\
\hline
\end{tabular}

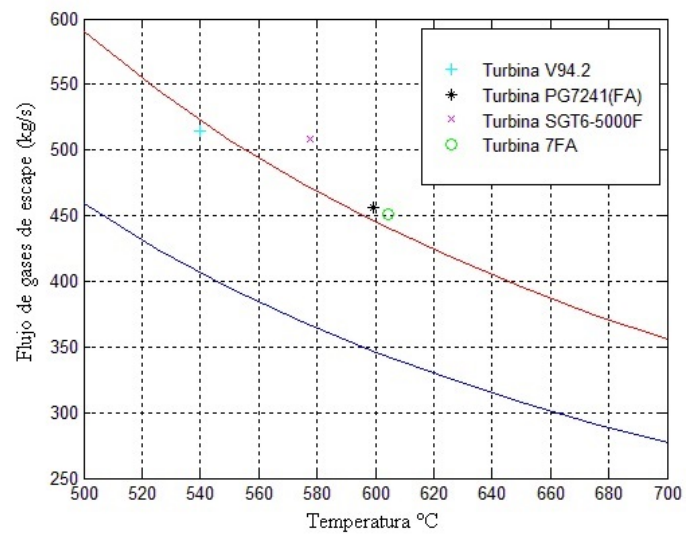

Figura 4. Turbinas seleccionadas en función de la temperatura y flujo de gases de escape. 
Como se pudo observar en la Figura 4, la turbina V94.2 es prácticamente descartada porque no satisface las necesidades térmicas de la planta. La turbina SGT6 es descartada por tener un exceso de flujo de gases de escape. Prácticamente las turbinas PG7241 y 7FA son las que están en paridad térmica con el proceso. Así que, con la consideración de que este sistema debe generar mínimo 4 veces más energía que la planta azucarera convencional, la turbina PG7241 es finalmente seleccionada.

Los datos de la turbina PG7241 presentados en la Tabla 2 están en las siguientes condiciones (Temperatura ambiente $=15{ }^{\circ} \mathrm{C}$ y altitud de $0 \mathrm{~m}$ ). Por lo tanto, estos datos de la turbina deben ser corregidos para las condiciones locales de la planta (Temperatura ambiente $=25{ }^{\circ} \mathrm{C}$ altitud de $530 \mathrm{~m}$ ), este paso es importante realizarlo ya que las condiciones ambientales influyen directamente en el rendimiento de la turbina.

\subsubsection{Cálculo del Pinch-Point}

La temperatura de los gases de escape a la salida de la caldera de recuperación tiene que ser corregida con el método del Pinch-Point de acuerdo con los criterios presentados en la Figura 5. Aquí se muestra que para no tener una impropiedad termodinámica, se debe tener una diferencia de temperatura mínima para el perfil de enfriamiento. Según Sue y Chuang, [20], este valor puede ser entre $10{ }^{\circ} \mathrm{C}$ y $30{ }^{\circ} \mathrm{C}$.

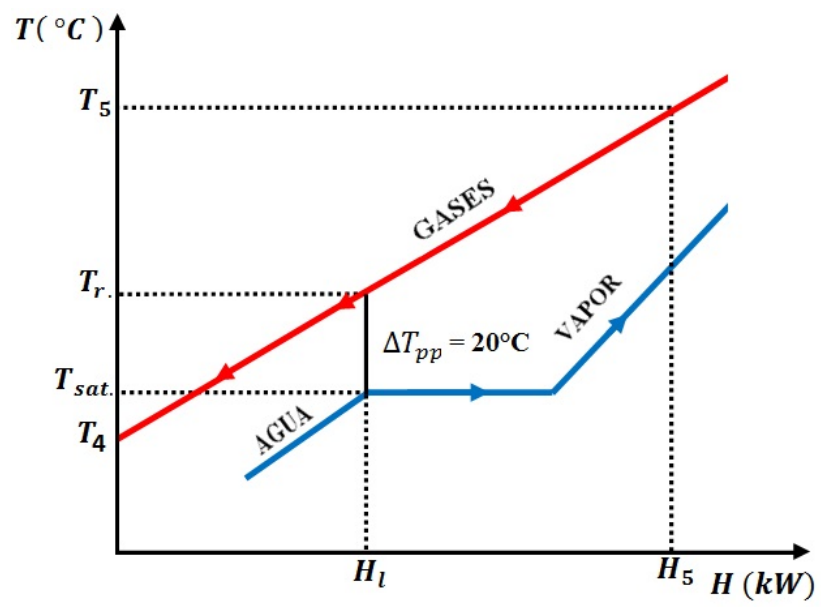

Figura 5. Pinch-Point.

La temperatura en el punto de rocío se determina con la Ecuación 10:

$$
T_{r}=T_{\text {sat }}+\Delta p p
$$

Para la temperatura de los gases de escape en la salida de la CR se usa las siguientes ecuaciones:

$$
T_{4}=T_{5}-\left[\frac{T_{5}-T_{r}}{H_{5}-H_{i}} \cdot H_{5}\right]
$$

$$
\begin{gathered}
H_{5}=\dot{m}_{5} \cdot C p_{G}\left(T_{5}\right) \cdot T_{5} \\
H_{l}=\dot{m}_{1} \cdot h_{l}
\end{gathered}
$$

Donde:

$\mathrm{h}_{\mathrm{l}}=$ entalpía del líquido saturado a la presión de operación de la caldera de recuperación $(\mathrm{kJ} / \mathrm{kg})$

\subsection{Análisis termodinámicos}

Los análisis técnicos realizados en el sistema de cogeneración están basados en la $1 .^{\mathrm{a}}$ y $2 .^{\mathrm{a}}$ ley de la termodinámica, es decir, análisis energéticos y exergéticos respectivamente.

\subsubsection{Análisis energético}

\section{Volumen de control del ciclo Brayton}

Para el cálculo del flujo del gas natural se tiene la Ecuación 14:

Heat rate $\cdot P_{\text {generador }}=P C I_{\text {gas natural }} \cdot \dot{m}_{8}$

El PCI del gas natural es $48300(\mathrm{~kJ} / \mathrm{kg})$ [21].

El flujo de aire a la entrada del compresor es calculado con las siguientes ecuaciones:

$$
\begin{gathered}
\dot{m}_{9}=\dot{m}_{5}-\dot{m}_{8} \\
\dot{m}_{9}=\dot{m}_{7}
\end{gathered}
$$

\section{Volumen de control del compresor}

La presión a la salida del compresor depende de la presión de entrada y de la relación de presiones. Esta presión es calculada con la Ecuación 17:

$$
R p=\frac{P_{7}}{P_{9}}
$$

La presión relativa en el punto 7 es calculada con la Ecuación 18:

$$
\operatorname{Pr}_{7}=\operatorname{Pr}_{9} \frac{P_{7}}{P_{9}}
$$

La entalpía del punto 7 es calculada con la Ecuación 19

$$
h_{7}=\frac{h_{7 s}-h_{9}}{\eta_{\text {comp }}}+h_{9}
$$

Por ley de conservación de la energía se tiene:

$$
\dot{m}_{6} \cdot h_{6}=\dot{m}_{7} \cdot h_{7}+P C I_{\text {gas natural }} \cdot \dot{m}_{8}
$$

Volumen de control de la turbina de gas

Para analizar la turbina de gas se tiene las ecuaciones: 


$$
\begin{gathered}
\operatorname{Pr}_{5}=\operatorname{Pr}_{6}\left(\frac{P_{5}}{P_{6}}\right) \\
\eta_{\text {isentrópica }}=\frac{h_{6}-h_{5}}{h_{6}-h_{5 s}}
\end{gathered}
$$

\section{Eficiencia energética}

Para el cálculo de la eficiencia de la turbina de gas se tiene la Ecuación 23:

$$
\eta_{\text {turbina }}=\frac{P_{\text {turbina }}}{P C I_{\text {gas natural }} \cdot \dot{m}_{8}}
$$

Donde:

$\dot{\mathrm{m}}_{8}=$ flujo de gas natural $(\mathrm{kg} / \mathrm{s})$

$\mathrm{PCI}_{\text {gas natural }}=$ poder calorífico inferior del gas natural $(\mathrm{kJ} / \mathrm{kg})$

$\mathrm{P}_{\text {turbina }}=$ potencia en la turbina $(\mathrm{kW})$

$\eta_{\text {turbina }}=$ eficiencia de la turbina (-)

Para el cálculo de la eficiencia total del sistema de cogeneración se tiene la Ecuación 24:

$$
\eta_{\text {sistema }}=\frac{P_{\text {turbina }}+\left[\left(h_{1}-h_{3}\right) \cdot \dot{m}_{1}\right]-P_{\text {bomba }}}{P C I_{\text {gas natural }} \cdot \dot{m}_{8}}
$$

Donde:

$\mathrm{h}_{1}=$ entalpía en el punto $1(\mathrm{~kJ} / \mathrm{kg})$

$\mathrm{h}_{3}=$ entalpía en el punto $3(\mathrm{~kJ} / \mathrm{kg})$

$\dot{\mathrm{m}}_{1}=$ flujo de vapor $(\mathrm{kg} / \mathrm{s})$

$\mathrm{P}_{\text {bomba }}=$ potencia consumida en la bomba $(\mathrm{kW})$

$\mathrm{P}_{\text {turbina }}=$ potencia en la turbina $(\mathrm{kW})$

$\eta_{\text {sistema }}=$ eficiencia del sistema (-)

\subsubsection{Análisis exergético}

El análisis exergético consiste en la evaluación cualitativa de las pérdidas a través del concepto de la exergía por medio de la aplicación de la segunda ley de la termodinámica. Un procedimiento básico para realizar este análisis es determinar los valores de entrada y salida de exergía para todos los componentes del sistema y la razón de la variación de esta para todo el proceso [16].

\section{Volumen de control del compresor}

En el compresor, el punto 9 es aire a temperatura y presión ambiente y el punto 7 es el aire comprimido (ver Figura 2).

Para encontrar la diferencia de entropía entre la entrada y la salida del compresor es aplicada la Ecuación 25 :

$$
\left(s_{7}-s_{9}\right)=\left(s_{7}^{0}-s_{9}^{0}\right)-R \ln \left(\frac{P_{7}}{P_{9}}\right)
$$

Donde:

$\mathrm{s}=$ entropía en los diferentes puntos del proceso $(\mathrm{kJ} / \mathrm{kg} \mathrm{K})$
$\mathrm{P}=$ presión en los diferentes puntos del proceso $(\mathrm{kPa})$

La diferencia de exergía en el compresor es calculada con la Ecuación 26:

$$
\Delta \psi_{\text {compresor }}=\left(h_{7}-h_{9}\right)-T_{0}\left(s_{7}-s_{9}\right)
$$

La exergía a la salida del compresor es obtenida aplicando la Ecuación 27:

$$
e x_{7}=\Delta \psi_{\text {compresor }} \cdot \dot{m}_{9}
$$

Donde:

$\mathrm{ex}_{7}$ : exergía en el punto $7(\mathrm{~kW})$

La irreversibilidad es calculada con la Ecuación 28:

$$
I_{7}=\frac{\left(h_{7}-h_{9}\right) \cdot \dot{m}_{7}}{\eta_{\text {compresor }}}-e x_{7}
$$

La eficiencia de segunda ley de la termodinámica en el compresor es calculada con la Ecuación 29:

$$
\eta_{\text {IIcompresor }}=\frac{\Delta \psi_{\text {compresor }}}{\frac{h_{7}-h_{9}}{\eta_{\text {compresor }}}}
$$

\section{Volumen de control de la CC}

En la CC, el punto 7 es aire comprimido, el punto 8 es gas natural y el punto 6 son gases de escape (ver Figura 2).

De la misma manera que en el compresor, en la CC de la planta se calcula la diferencia de entropía entre la entrada y salida de la CC, el cambio de exergía, la exergía en el punto 6 y la eficiencia de la segunda ley de la termodinámica. Para el cálculo se aplican las ecuaciones:

$$
\begin{gathered}
\left(s_{6}-s_{7}\right)=\left(s_{6}^{0}-s_{7}^{0}\right)-R \ln \left(\frac{P_{6}}{P_{7}}\right) \\
\Delta \psi_{\text {cam.comb. }}=\left(h_{6}-h_{7}\right)-T_{0}\left(s_{6}-s_{7}\right) \\
\Delta e x=\Delta \psi_{\text {cam.comb. }} \dot{m}_{9} \\
e x_{6}=\Delta e x+e x_{7} \\
\eta_{\text {IIcam-comb. }}=\frac{e x_{6}}{e x_{7}+e x_{8}}
\end{gathered}
$$

\section{Volumen de control de la turbina}

En la turbina, el punto 6 es la entrada de los gases de escape a la turbina y el punto 5 es la salida (ver Figura 2).

De la misma manera que en el compresor y la CC las siguientes ecuaciones son aplicadas a la turbina de gas. 


$$
\begin{gathered}
\left(s_{6}-s_{5}\right)=\left(s_{6}^{0}-s_{5}^{0}\right)-R \ln \left(\frac{P_{6}}{P_{5}}\right) \\
\Delta \psi_{\text {turbina }}=\left(h_{6}-h_{5}\right)-T_{0}\left(s_{6}-s_{5}\right) \\
\Delta e x=\Delta \psi_{\text {turbina }} \cdot \dot{m}_{9} \\
e x_{5}=e x_{6}-\Delta e x \\
\eta_{\text {IIturbina }}=\frac{W_{\text {total }}}{\Delta_{e x}}
\end{gathered}
$$

\section{Volumen de control en la CR}

En la CR, el punto 5 es la entrada de los gases de escape y el punto 4 es la salida de los gases de escape al ambiente. Por el otro lado de la CR, el punto 3 es agua y el punto 1 es vapor sobrecalentado (ver Figura 2).

La exergía de los gases de escape es calculada con la Ecuación 40:

$$
e x_{4}=\left[c_{p}\left(T_{4}-T_{0}\right)-T_{0}\left(c_{p} \ln \frac{T_{4}}{T_{0}}-R \ln \frac{P_{4}}{P_{0}}\right)\right] \dot{m}_{4}
$$

La entropía en el punto 4 es determinada con la Ecuación 41:

$$
s_{4}-s_{0}=c_{p} \ln \frac{T_{4}}{T_{0}}-R \ln \frac{P_{4}}{P_{0}}
$$

El cambio de exergía entre los puntos 4 y 5 son calculados con las ecuaciones:

$$
\begin{gathered}
\Delta e x_{4-5}=\left\lfloor c_{p}\left(T_{4}-T_{5}\right)-T_{0}\left(s_{4}-s_{5}\right)\right\rfloor \cdot \dot{m}_{5} \\
s_{4}-s_{5}=c_{p} \ln \frac{T_{4}}{T_{5}}-R \ln \frac{P_{4}}{P_{5}}
\end{gathered}
$$

El cambio de exergía entre los puntos 1 y 3 son calculados con las ecuaciones:

$$
\begin{gathered}
\Delta \psi_{1-3}=\left(h_{1}-h_{3}\right)-T_{0}\left(s_{1}-s_{3}\right) \\
\Delta e x_{1-3}=\Delta \psi_{1-3} \cdot \dot{m}_{1}
\end{gathered}
$$

La eficiencia de la segunda ley en la CR es calculada con la Ecuación 46:

$$
\eta_{I I C R}=\frac{\Delta e x_{1-3}}{-\Delta e x_{4-5}}
$$

\section{Volumen de control de la bomba}

En la bomba, los puntos 2 y 3 son agua, en este caso el punto 3 con una presión mayor que el punto 2 (ver Figura 2). 47:

Para la exergía en el punto 3 se tiene la Ecuación

$$
e x_{3}=\dot{m}_{3} \cdot\left[\left(h_{3}-h_{2}\right)-T_{0} \cdot\left(s_{3}-s_{2}\right)\right]
$$

Para la irreversibilidad en el punto 3 se tiene la Ecuación 48:

$$
I_{3}=\left(\left(h_{3}-h_{2}\right) \cdot \dot{m}_{3}\right)-e x_{3}
$$

Para calcular la eficiencia de la segunda ley en la bomba se tiene la Ecuación 49:

$$
\eta_{\text {IIbomba }}=\frac{e x_{3}}{P_{b o m b a}}
$$

\section{Eficiencia exergética del sistema}

La eficiencia total de la segunda ley de la termodinámica es calculada con la Ecuación 50:

$$
\eta_{I \text { Ibomba }}=\frac{P_{\text {turbina }}+\Delta e x_{1-3}-P_{\text {bomba }}}{P C I_{\text {gas natural }} \cdot \dot{m}_{8}}
$$

\subsection{Análisis económico}

$\mathrm{El}$

cidad y vapor producido por el sistema de cogeneración. Para este análisis fue considerado el valor de los equipos, valor de manutención, costo de operación de la planta, tasa de impuestos, tiempo de retorno de la inversión y el costo del gas natural.

\subsubsection{Costo de los equipos}

Según Castro [16] y Silveira [22] para calcular el valor de los equipos se aplican las siguientes ecuaciones:

Inversión en la turbina de gas:

$$
I_{T G}=\left(\frac{U S \$}{k W} \cdot E_{P T G}\right)
$$

Donde:

$\frac{\mathrm{US} \$}{\mathrm{~kW}}=234 \frac{\mathrm{US} \$}{\mathrm{~kW}}[19]$

$\mathrm{E}_{\mathrm{PTG}}=$ potencia producida por la turbina $(\mathrm{kW})$

Inversión en la caldera de recuperación:

$I_{C R}=4745 \cdot\left(\frac{h_{1}}{\log \left(T_{5}-T_{1}\right)}\right)+11820 \cdot \dot{m}_{1}+658 \cdot \dot{m}_{5}$

\section{Costo de la energía eléctrica y vapor}

Para el cálculo del costo de la energía eléctrica y el vapor producido se tienen las ecuaciones 53 y 54 respectivamente:

$$
C_{\text {elec }}=\frac{I_{T G} \cdot f}{H \cdot E_{P T G}}+\frac{C_{\text {comb }}\left(E_{\text {comb }}-E_{c}-\frac{P_{e r}}{2}\right)}{E_{p}} \ldots
$$




$$
\begin{array}{r}
C_{v}=\frac{I_{C R} \cdot f}{H \cdot Q_{p}}+\frac{C_{c o m b}\left(E_{c o m b}-E_{c}-\frac{P_{e r}}{2}\right)}{E_{v}} \ldots \\
+C m_{C R}+C o
\end{array}
$$

Donde:

$\mathrm{C}_{\text {comb }}=$ costo del combustible $\frac{\mathrm{US} \$}{\mathrm{kWh}}$

$\mathrm{C}_{\text {elec }}=$ costo de electricidad $\frac{\mathrm{US} \$}{\mathrm{kWh}}$

$\mathrm{Cm}=$ costo mantenimiento de la $\mathrm{TG} \frac{\mathrm{US} \$}{\mathrm{kWh}}$

$\mathrm{Cm}_{\mathrm{CR}}=$ costo mantenimiento de la $\mathrm{CR} \frac{\mathrm{US} \$}{\mathrm{kWh}}$

$\mathrm{C}_{\mathrm{v}}=$ costo de la producción de vapor $\frac{\mathrm{USS}}{\mathrm{kWh}}$

Co $=$ costo del personal de operación $\frac{\mathrm{US} \$}{\mathrm{kWh}}$

$\mathrm{E}_{\mathrm{comb}}=$ potencia del combustible $(\mathrm{kW})$

$\mathrm{E}_{\mathrm{p}}=$ electricidad producida $(\mathrm{kWh}) \frac{\mathrm{US} \$}{\mathrm{kWh}}$

$\mathrm{E}_{\mathrm{v}}=$ vapor producido $(\mathrm{kWh}) \frac{\mathrm{US} \$}{\mathrm{kWh}}$

Para el factor de anualidad se tiene la Ecuación 55 y para el valor del capital la Ecuación 56 :

$$
\begin{gathered}
f=\frac{q^{k} \times(q-1)}{q^{k}-1} \\
q=1+\frac{r}{100}
\end{gathered}
$$

Donde:

$\mathrm{k}=$ período de amortización (años)

$\mathrm{r}=$ tipo de interés anual (\%)

\section{Resultados y discusión}

\subsection{Resultados del análisis termodinámico}

Los resultados del análisis termodinámico se muestran en las Tablas 3 y 4 . En la Tabla 3 se presentan las eficiencias energéticas y exergéticas.

Tabla 3. Eficiencias energéticas y exergéticas

\begin{tabular}{ccc}
\hline Equipo & $\begin{array}{c}\text { Eficiencia } \\
\text { Energética }\end{array}$ & $\begin{array}{c}\text { Eficiencia } \\
\text { exergética }\end{array}$ \\
\hline Compresor & - & $86 \%$ \\
$\begin{array}{c}\text { Cámara de } \\
\text { combustión } \\
\text { Turbina }\end{array}$ & - & $76 \%$ \\
$\begin{array}{c}\text { de gas } \\
\text { Caldera de }\end{array}$ & $39 \%$ & $79 \%$ \\
$\begin{array}{c}\text { Recuperación } \\
\text { Bomba }\end{array}$ & - & $59 \%$ \\
Eficiencia \\
total
\end{tabular}

De la Tabla 3 se puede ver como la eficiencia total del sistema (energética) es casi dos veces mayor que la eficiencia de la turbina, esto es debido a que se usa los gases de escape de la turbia de gas para generar vapor (cogeneración).
Los trabajos de Castro [16] y Paula Santos et al. [23] muestran estudios técnicos similares en sistemas de $\mathrm{CC}$ en donde se obtuvieron eficiencias superiores al $50 \%$, lo cual corrobora los resultados de este trabajo.

Por otro lado, en la Tabla 4 se presentan los parámetros termodinámicos para cada punto del sistema de cogeneración.

Tabla 4. Resumen del análisis energético y exergético

\begin{tabular}{ccccccc}
\hline$\#$ & $\begin{array}{c}\mathbf{m} \\
\mathbf{k g} / \mathbf{s}\end{array}$ & $\begin{array}{c}\mathbf{P} \\
\mathbf{k P a}\end{array}$ & $\begin{array}{c}\mathbf{T} \\
{ }^{\circ} \mathbf{C}\end{array}$ & $\begin{array}{c}\mathbf{h} \\
\mathbf{k J} / \mathbf{k g}\end{array}$ & $\begin{array}{c}\mathbf{s} \\
\mathbf{k J} / \mathbf{k g} \cdot \mathbf{K}\end{array}$ & $\begin{array}{c}\mathbf{e x} \\
\mathbf{k W}\end{array}$ \\
\hline 1 & 50 & 6860 & 530 & 3484 & 6,9 & 67871,5 \\
2 & 50 & 245 & 126,8 & 532,5 & 2,27 & 0 \\
3 & 50 & 7860 & 128,1 & 543,4 & 1,6 & 254,44 \\
4 & 419 & 94,3 & 227,9 & 503 & 2,31 & 25020 \\
5 & 419 & 94,3 & 634,6 & 941,6 & 2,92 & 112071 \\
6 & 419 & 1462 & 1208 & 1612 & 2,73 & 417112 \\
7 & 408 & 1462 & 407,5 & 692,5 & 1,75 & 153508 \\
8 & 8,1 & 94,3 & 25 & - & - & 393611 \\
9 & 408 & 94,3 & 25 & 298,6 & 1,69 & 0 \\
\hline
\end{tabular}

Para una mejor representación de los resultados de la parte energética se presenta en la Figura 6 el diagrama de Sankey. Este diagrama representa los flujos de energía desde la entrada del sistema hasta la salida del mismo, así como las pérdidas que tiene cada componente.

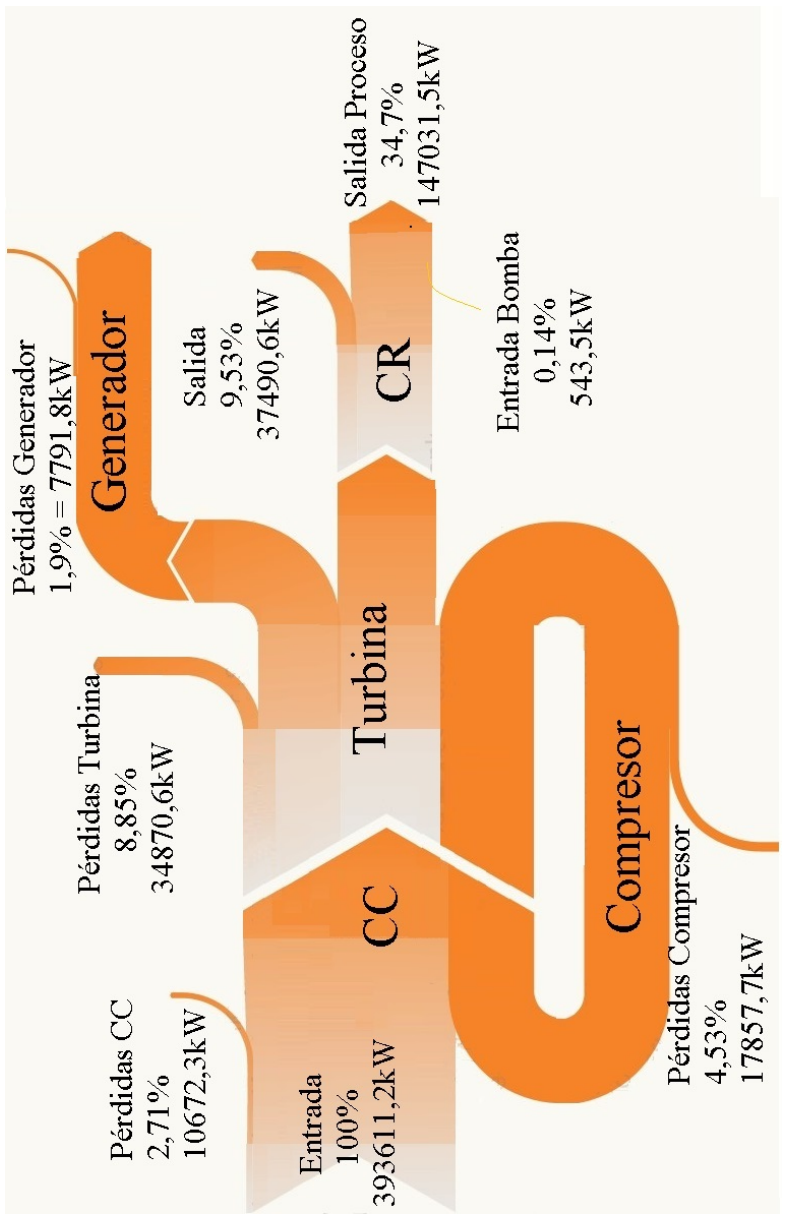

Figura 6. Diagrama de Sankey 
De la misma manera, para el análisis exergético se presenta el diagrama de Grassman en la Figura 7.

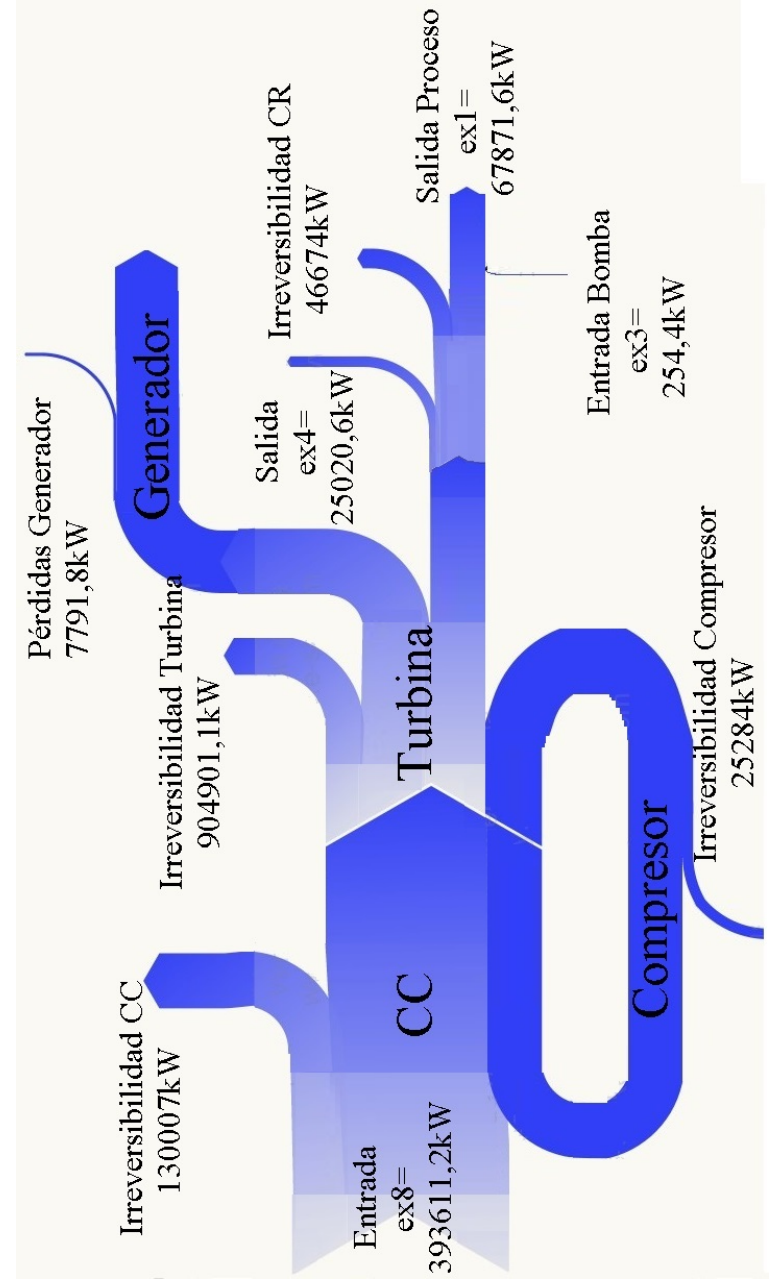

Figura 7. Diagrama de Grassman

Los resultados de este análisis son los costos de la energía eléctrica y del vapor producido por la planta considerando una tasa de impuestos de 4,8 y $12 \%$ y un periodo de amortización de hasta 20 años. También se considera que la planta trabaja solamente 4000 horas, pues se pretende hacer el análisis en las condiciones más drásticas.

Por un lado la Figura 8 presenta el costo de la energía eléctrica producida.

Como se puede observar en la Figura 8 el valor más alto (precio) de la energía eléctrica producida está en 0,108 US $\$ / \mathrm{kWh}(\mathrm{r}=12 \%)$ mientras que el más bajo está en 0,103 US $\$ / \mathrm{kWh}(\mathrm{r}=4 \%)$, esto cuando la planta está recién instalada.

Luego de 20 años este valor se reduce en aproximadamente $30 \%$.

Finalmente, la Figura 9 presenta el costo del vapor producido por el proceso.

De la misma manera, como se observó en la Figura 9, el precio más alto del vapor producido es de 0,0682
US\$ $/ \mathrm{kWh}(\mathrm{r}=12 \%)$ y el más barato es de 0,0678 US\$/kWh $(\mathrm{r}=4 \%)$ cuando la planta está recién instalada. Estos valores se reducen en aproximadamente $5 \%$ luego de 20 años.

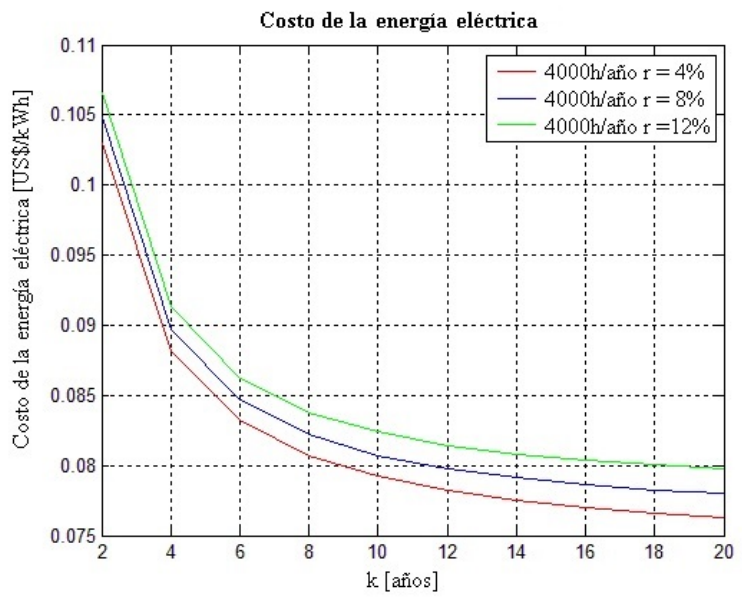

Figura 8. Costo de la energía eléctrica producida

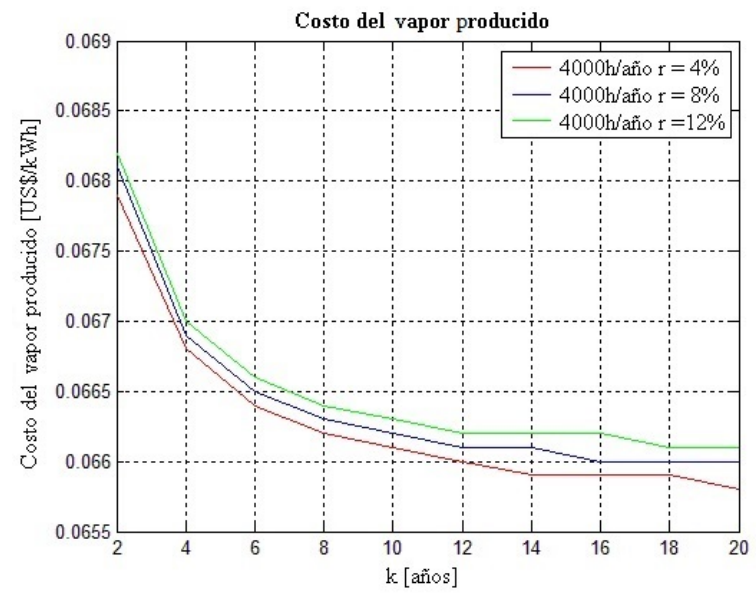

Figura 9. Costo del vapor producido

\subsection{Discusión del análisis económico}

De acuerdo con la Agencia Nacional de Energía Eléctrica en Brasil, el precio de venta de la energía eléctrica en el año 2015 fue de 191,11 R\$/MWh. Considerando la tasa de cambio de dólares a reales en el primer trimestre de este mismo año (1 US\$ $=2 \mathrm{R} \$)$, el precio de la energía es de 0,095 US\$/kWh. Así, se puede considerar como aceptable la implementación de este sistema ya que además de producir electricidad se está produciendo vapor. Asimismo, este precio se puede mejorar considerablemente si la planta trabaja 7200 h/año y si se gasifica el bagazo de caña para producir gas de síntesis y poder sustituir el uso del gas natural; estas dos últimas hipótesis están siendo estudiadas y se presentarán resultados en próximas publicaciones. 


\section{Conclusiones}

En este trabajo se realizó el estudio técnico basado en la primera y segunda ley de la termodinámica y el estudio económico de un sistema de cogeneración. Por un lado, del análisis energético se puede ver que la planta tiene una capacidad de producción de potencia eléctrica de 148,045 MW y térmica de 147,031 MW. De la misma manera, este análisis muestra que la eficiencia total del sistema es aproximadamente dos veces mayor $(67 \%)$ que la eficiencia de la turbina, esto es debido a que se usa los gases de escape de la turbia para generar vapor.

Por otro, el análisis exergético muestra que la capacidad de potencia eléctrica es la misma, mientras que la capacidad térmica disminuye para $67 \mathrm{MW}$, esto debido a las irreversibilidades. En este caso la eficiencia total del sistema es de $56 \%$. En el análisis económico se pudo ver que los precios de la electricidad y el vapor producido son de 0,105 y 0,068 (US $\$ / \mathrm{kWh}$ ) cuando la planta es inicialmente instalada; este costo se ve reducido en $30 \%$ para el caso de la energía eléctrica y $5 \%$ para el caso del vapor luego de 20 años.

Finalmente, en la parte del dimensionamiento se puede concluir que el flujo de los gases de escape de la turbina seleccionada en paridad térmica reveló ser suficiente para abastecer las necesidades eléctricas y térmicas del sistema de cogeneración sin necesidad de quema suplementaria de combustible.

Dando continuidad a este estudio, se pretende realizar el análisis termodinámico del proceso de gasificación del bagazo de caña para producir gas de síntesis con el objetivo de sustituir el uso del gas natural y abaratar el costo de la producción de energía.

\section{Referencias}

[1] D. Tomofuji, Y. Morimoto, E. Sugiura, T. Ishii, and A. Akisawa, "The prospects of the expanded diffusion of cogeneration to 2030 Study on new value in cogeneration," Applied Thermal Engineering, vol. 114, Supplement C, pp. 1403-1413, 2017. [Online]. Available: https: //doi.org/10.1016/j.applthermaleng.2016.09.071

[2] N. M. Isa, C. W. Tan, and A. Yatim, "A comprehensive review of cogeneration system in a microgrid: A perspective from architecture and operating system," Renewable and Sustainable Energy Reviews, vol. 81, Part 2, pp. 2236-2263, 2018. [Online]. Available: https://doi.org/10.1016/j.rser.2017.06.034

[3] R. Carapellucci and A. Milazzo, "Repowering combined cycle power plants by a modified STIG configuration," Energy Conversion and Management, vol. 48, no. 5, pp. 1590-1600, 2007. [Online]. Available: https://doi.org/10.1016/j.enconman.2006.11.024

[4] A. Rovira, C. Sánchez, M. Muñoz, M. Valdés, and M. Durán, "Thermoeconomic optimisation of heat recovery steam generators of combined cycle gas turbine power plants considering off-design operation," Energy Conversion and Management, vol. 52, no. 4, pp. 1840-1849, 2011. [Online]. Available: https://doi.org/10.1016/j.enconman.2010.11.016

[5] D. Mahto and S. Pal, "Thermodynamics and thermo-economic analysis of simple combined cycle with inlet fogging," $A p$ plied Thermal Engineering, vol. 51, no. 1, pp. 413-424, 2013. [Online]. Available: https: //doi.org/10.1016/j.applthermaleng.2012.09.003

[6] G. Heyen and B. Kalitventzeff, "A comparison of advanced thermal cycles suitable for upgrading existing power plant," $A p$ plied Thermal Engineering, vol. 19, no. 3, pp. 227-237, 1999. [Online]. Available: https://doi.org/10.1016/S1359-4311(98)00044-1

[7] J. Silveira and C. Tuna, "Thermoeconomic analysis method for optimization of combined heat and power systems. Part I," Progress in Energy and Combustion Science, vol. 29, no. 6, pp. 479-485, 2003. [Online]. Available: https://doi.org/10.1016/S0360-1285(03)00041-8

[8] —, "Thermoeconomic analysis method for optimization of combined heat and power systems-Part II," Progress in Energy and Combustion Science, vol. 30, no. 6 , pp. $673-678,2004$. [Online]. Available: https://doi.org/10.1016/j.pecs.2003.06.001

[9] M. A. Kordlar and S. Mahmoudi, "Exergeoconomic analysis and optimization of a novel cogeneration system producing power and refrigeration," Energy Conversion and Management, vol. 134, Supplement C, pp. 208-220, 2017. [Online]. Available: https://doi.org/10.1016/j.enconman.2016.12.007

[10] M. Gambini and M. Vellini, "High efficiency cogeneration: Electricity from cogeneration in CHP plants," Energy Procedia, vol. 81, Supplement C, pp. 430-439, 2015, [Online]. Available: https://doi.org/10.1016/j.egypro.2015.12.117

[11] D. Gvozdenac, B. G. Urošević, C. Menke, D. Urošević, and A. Bangviwat, "High efficiency cogeneration: $\mathrm{CHP}$ and non-CHP energy," Energy, vol. 135, Supplement C, pp. 269-278, 2017. [Online]. Available: https://doi.org/10.1016/j.energy.2017.06.143 
[12] B. B. Kanbur, L. Xiang, S. Dubey, F. H. Choo, and F. Duan, "Thermoeconomic assessment of a micro cogeneration system with LNG cold utilization," Energy, vol. 129, Supplement C, pp. 171-184, 2017. [Online]. Available: https://doi.org/10.1016/j.energy.2017.04.071

[13] Z.-G. Sun, "Energy efficiency and economic feasibility analysis of cogeneration system driven by gas engine," Energy and Buildings, vol. 40, no. 2, pp. 126-130, 2008. [Online]. Available: https://doi.org/10.1016/j.enbuild.2007.01.013

[14] A. Abusoglu and M. Kanoglu, "Exergetic and thermoeconomic analyses of diesel engine powered cogeneration: Part 1 - Formulations," Applied Thermal Engineering, vol. 29, no. 2, pp. 234-241, 2009. [Online]. Available: https: //doi.org/10.1016/j.applthermaleng.2008.02.025

[15] R. Passolongo, "Avaliação termodinâmica, termoeconômica e econômica da integração de sistemas de gaseificação da biomassa em uma usina sucroalcooleira," Master's thesis, Universidade Estadual Paulista, Faculdade de Engenharia de Ilha Solteira, 2011. [Online]. Available: https://goo.gl/uj3k4s

[16] I. A. de Castro Villela, "Combined fault detection and classification of internal combustion engine using neural network," Ph.D. dissertation, Universidade Estadual Paulista, Facultade de Engenharia de Guaratinguetá, 2007. [Online]. Available: https://goo.gl/w4hmYA
[17] Y. Çengel and M. Boles, Termodinámica (8a. ed.): McGraw Hill, 2015. [Online]. Available: https://goo.gl/YoY7HE

[18] Uniongas. (2017) Chemical composition of natural gas. [Online]. Available: https://goo.gl/1ME1zf

[19] GTW. (2012) Gasturbine world handbook. Pequet. [Online]. Available: https://goo.gl/SNsJv1

[20] D.-C. Sue and C.-C. Chuang, "Engineering design and exergy analyses for combustion gas turbine based power generation system," Energy, vol. 29, no. 8, pp. 1183-1205, 2004. [Online]. Available: https://doi.org/10.1016/j.energy.2004.02.027

[21] Unitrove. (2017) Natural gas calorific value calculator. [Online]. Available: https://goo.gl/rU6Uir

[22] J. L. Silveira, "Cogeração disseminada para pequenos usuarios: estudo de casos para o setor terciario," Ph.D. dissertation, Universidade Estadual de Campinas, Facultade de Engenharia Mecânica, 1994. [Online]. Available: https://goo.gl/oBjSeK

[23] C. F. de Paula Santos, R. F. Silva Paulino, C. E. Tuna, J. L. Silveira, and F. H. Mayworm de Araújo, "Thermodynamics analysis and ecological efficiency of a combined cycle power plant," Engenharia Térmica, vol. 13, no. 2, pp. 3-8, 2014. [Online]. Available: https://goo.gl/9Ei1Se 


\title{
ANÁLISIS DE MEDIDAS DE AHORRO DE ENERGÍA EN UNA EMPRESA DE PRODUCCIÓN
}

\section{ANALYSIS OF ENERGY SAVING MEASURES IN A PRODUCTION COMPANY}

\author{
Luisa Salazar ${ }^{1, *}$, Víctor Guzmán², Alexander Bueno ${ }^{1}$
}

\section{Resumen}

Este trabajo analiza las medidas de ahorro de energía posibles en una empresa de producción, con la finalidad de establecer recomendaciones que contribuyan al uso eficiente de la energía. Esta investigación es de tipo descriptivo-documental, sustentada en un trabajo de campo, en donde se analizan los diferentes procesos de producción y servicios industriales. Utilizando datos suministrados de horas de trabajo, se realiza una estimación del consumo mensual para determinar el impacto de los procesos en la facturación de electricidad; el análisis se restringe al cálculo de índices de consumo $(\mathrm{t} / \mathrm{kWh})$ con lo que se puede priorizar el uso de los equipos más eficientes sobre otros que realicen las mismas funciones. El análisis de la característica de consumo arroja puntos óptimos de consumo con los cuales se establece un gráfico de energía vs. producción llamado meta. Se obtiene como resultado que aplicando todas las acciones propuestas, el consumo mensual disminuiría en $138024,84 \mathrm{kWh}$, lo que representa un ahorro de energía del orden del $25 \%$ respecto con el consumo promedio actual.

Palabras clave: ahorro de energía, caracterización, meta, producción.

\section{Abstract}

This work analyzes possible measures of energy saving in a production company, with the purpose of establishing recommendations that contribute to the efficient use of energy. This research is descriptivedocumentary, based on fieldwork, where the different production processes and industrial services were analyzed. Using data supplied from working hours, an estimate of monthly consumption was made to determine the impact of processes on electricity billing. The analysis was restricted to the calculation of consumption index (Ton $/ \mathrm{kWh}$ ), which allows the use of the most efficient equipment to be prioritized over others performing the same functions. The analysis of the consumption characteristic yields optimum consumption points which allow establishing a graph of Energy vs Production called Goal. The result is that by applying all the proposed actions, the monthly consumption would decrease by $138,024.84 \mathrm{kWh}$, representing an energy saving of around $25 \%$ with respect to the current average consumption.

Keywords: Energy Saving, Characterization, Goal, Production

\footnotetext{
1,* Departamento de Conversión y Transporte de Energía, Universidad Simón Bolívar - Venezuela. Autor para correspondencia luisalazar@usb.ve. (D) http://orcid.org/0000-0002-0824-8516

${ }^{2}$ Departamento de Electrónica y Circuitos, Universidad Simón Bolívar - Venezuela.

(D) http://orcid.org/0000-0002-7029-5727, (D) http://orcid.org/0000-0001-9167-522X
}

Recibido: 12-02-2017, aprobado tras revisión: 17-09-2017

Forma sugerida de citación: Salazar, L.; Guzmán, V.; Bueno, A. (2018). «Análisis de medidas de ahorro de energía en una empresa de producción». InGEnIUs. N. ${ }^{\circ} 19$, (enero-junio). pp. 40-50. DoI: https://doi.org/10.17163/ings.n19.2018.04. 


\section{Introducción}

La evolución del hombre en la Tierra ha sido posible en gran parte por el desarrollo de tecnologías que hacen mucho más fácil su día a día. El ser humano pasó de emplear su fuerza muscular y la de animales domesticados al uso de diversas fuentes energéticas como viento, agua, fuego, vapor, entre otros, llegando al esquema energético actual, en donde la quema de combustibles fósiles desempeña un papel preponderante; cabe destacar que esta fuente energética es finita y contaminante en alto grado.

Muchas son las plantas generadoras de electricidad que la usan como fuerza motriz para poder abastecer la demanda eléctrica, esto implica una alta emisión de gases a la atmósfera terrestre que afectan el ecosistema. Además de la contaminación, hay que tener en cuenta que la escasez de petróleo en un futuro representará un gran problema energético mundial, por lo que la disminución del consumo eléctrico implica ahorro de esta importante fuente de energía.

El inicio del tercer milenio representa para la humanidad la encrucijada de una nueva elección energética, frente al agotamiento de los combustibles fósiles por una parte, pero sobre todo, por la amenaza de una catástrofe ecológica, al rebasarse los límites de la capacidad del planeta para asimilar su impacto [1].

En líneas generales, la sociedad venezolana no se ha caracterizado por tener una cultura de ahorro energético, debido a que en el pasado las grandes riquezas energéticas del país han sustentado una oferta muy por encima de la demanda nacional en cuanto al servicio eléctrico se refiere. Sin embargo, en los últimos años, la aparición de largos periodos de sequía ha afectado directamente la principal fuente de generación de electricidad del país que es la hidráulica (Figura 1).

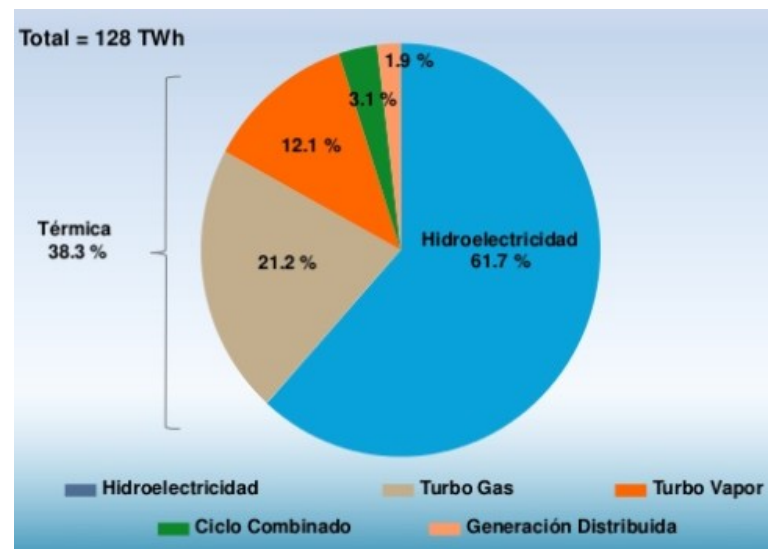

Figura 1. Balance de energía en Venezuela por tipo de planta, estimaciones 2015 [2].

Además, el crecimiento exponencial de la población y de los diversos sectores industriales y comerciales, trae como consecuencia el aumento la demanda eléctrica (Figura 2). Ambos factores han dado un giro, no solo a la situación energética, sino también al impacto ambiental que esta produce y a la reducción de las reservas de petróleo.

Hoy en día no existe la capacidad de generar la potencia eléctrica suficiente para abastecer a toda la población si se mantiene el nivel de consumo tradicional, es por ello por lo que surge la necesidad de implantar planes de concientización acerca del uso eficiente de la energía, con el propósito de disminuir el consumo innecesario de la misma.

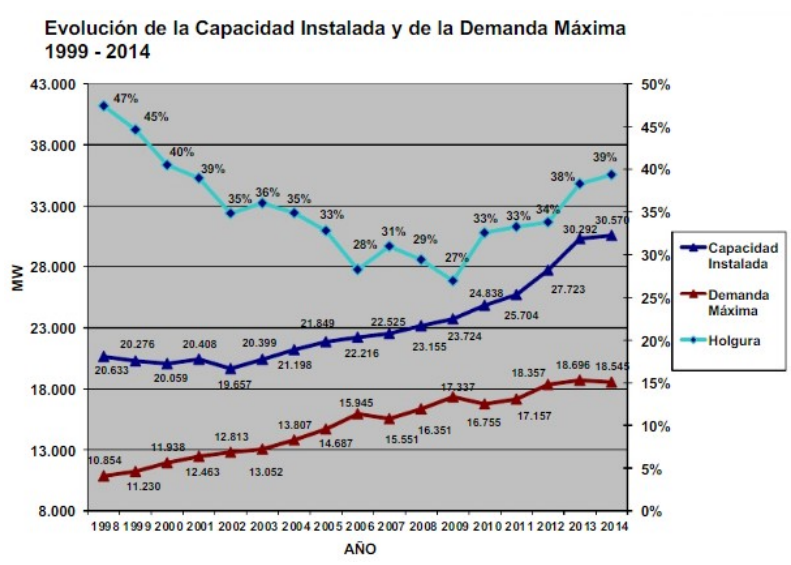

Figura 2. Demanda de potencia promedio del Sistema Eléctrico Nacional (SEN) periodo 1999-2014 [2].

La empresa motivo de estudio, Montana Gráfica, filial del Grupo Corimon Pintura C. A., se dedica a la elaboración de empaques y etiquetas flexibles para diversos productos de alto consumo como alimentos, bebidas, golosinas, entre otros. Se encuentra ubicada en Mariara, estado Carabobo, Venezuela y cuenta con unos $30000 \mathrm{~m}^{2}$ de construcción.

Se alimenta por una línea trifásica de $13.8 \mathrm{kV}$ suministrada por la empresa nacional de servicio de electricidad Corporación Eléctrica (Corpoelec), la cual se conecta a tres subestaciones internas, equipadas con un total de siete transformadores trifásicos con una potencia nominal de $6590 \mathrm{kVA}$; estos reducen la tensión a $460 \mathrm{~V}, 440 \mathrm{~V}$ o $220 \mathrm{~V}$, dependiendo de la unidad, según el diagrama unifilar mostrado en la Figura 3.

La planta tiene un consumo mensual promedio de unos $637000 \mathrm{kWh}$, y su demanda contratada se ubica en $1860 \mathrm{kVA}$. Entra en la clasificación de alto consumidor, ya que factura por encima de $30000 \mathrm{kWh} / \mathrm{mes}$ [3]. Se trata de una fábrica con un consumo energético considerable en sistemas activos, tales como alumbrado de alto consumo y equipos que son relativamente ineficientes en comparación con nuevas tecnologías; las decisiones y estrategias que ha tomado la gerencia en cuanto a la optimización del consumo no han sido tan 


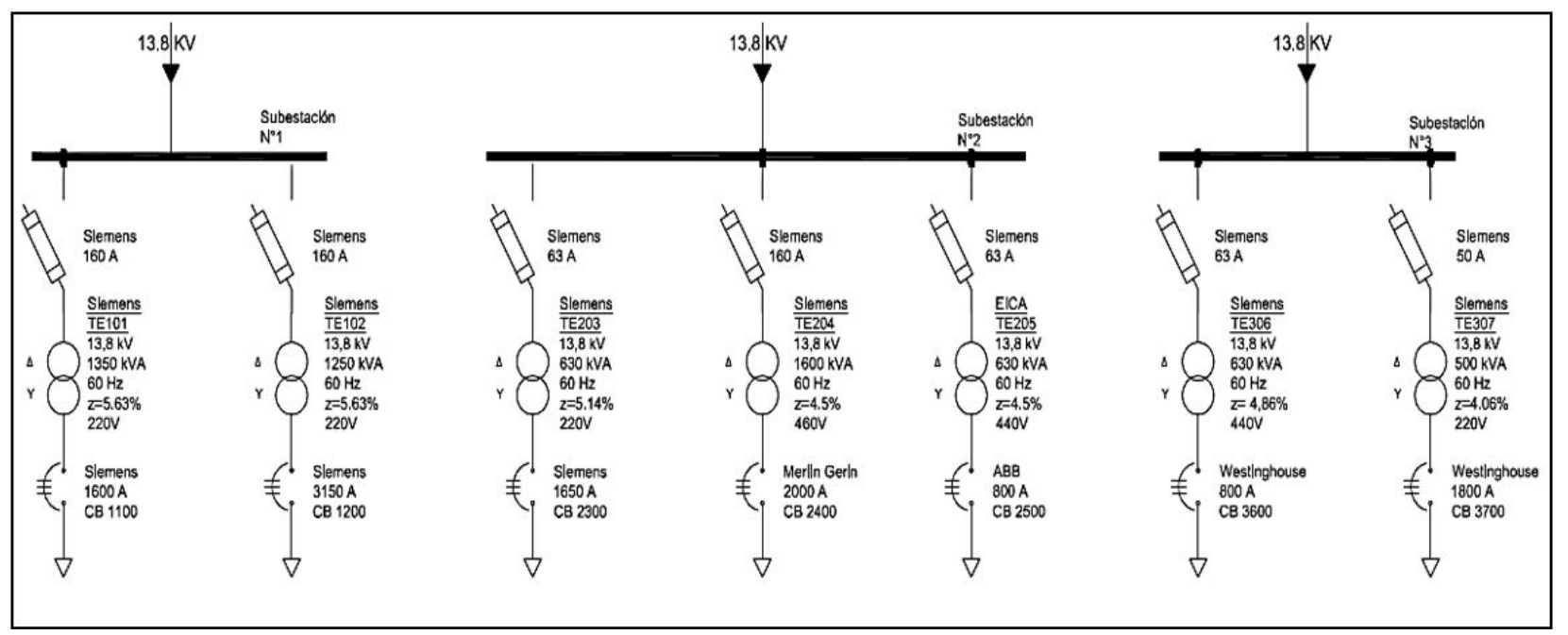

Figura 3. Diagrama unifilar general [3].

efectivas como se esperaba, por lo que se ha comisionado este estudio para lograr un efecto significativo en cuanto al uso eficiente de la energía.

\section{Desarrollo y método}

El consumo y la disponibilidad de energía son factores preponderantes en los costos de producción. Por este motivo se pone de manifiesto en el sector manufacturero y de servicios la necesidad de realizar una cuidadosa gestión de la energía para aumentar la competitividad [4].

La eficiencia energética se puede definir como la capacidad de un uso, equipo, instalación o proceso para realizar su función con el menor consumo energético posible, de la misma forma se puede entender el ahorro de energía como la disminución del consumo de energía primaria de un centro de consumo de energía por la implementación de medidas de índole técnica o no técnica [4], la aplicación de estos conceptos en la gestión de la energía de la empresa aumenta la productividad y competitividad de esta.

Esto ha hecho necesario que las empresas planteen sus propios «modelos de gestión» de la energía para motivar e incentivar un desarrollo de la nueva cultura organizacional para el uso eficiente de la energía.

Esta es por diseño una investigación documental, descriptiva y de campo definida por actividades enmarcadas en la recopilación de información que permiten realizar la caracterización energética de la empresa Montana Gráfica, filial del Grupo Corimon Pintura C. A., mediante la aplicación de herramientas de gestión que forman parte del procedimiento presentado en la Norma Internacional ISO 50001, referido a la revisión energética, que determina el desempeño energético de la organización basada en datos y otro tipo de información, orientada a la identificación de oportunidades de mejora, contemplada en uno de los requerimientos medulares de la ISO 50001, la planificación energética, que implica una revisión de las actividades de la empresa que puedan afectar el desempeño energético [5], para la implantación de un sistema de gestión de la energía.

Para llevar a cabo la caracterización energética de la empresa se desarrollaron las siguientes actividades [5]:

1. Definir el diagrama energético productivo: diagrama de flujo de los principales procesos productivos.

2. Realizar el censo de carga: consumos por áreas y equipos representativos.

3. Calcular el diagrama de Pareto y estratificación: $20 \%$ de los equipos y áreas que consumen aproximadamente el $80 \%$ de la energía eléctrica.

4. Proponer diagramas de control: variación simultánea del consumo energético con la producción realizada en el tiempo, para áreas y equipos. Comprenden la elaboración del gráfico de control, gráfico energía producción vs. tiempo, gráfico energía vs. producción, gráfico meta vs. producción y gráfico de índice de consumo vs. producción.

A continuación se presenta el desarrollo de las actividades:

\subsection{Diagrama energético productivo}

Es de suma importancia conocer las áreas de la planta y los distintos procesos que se realizan en ella, así como los equipos y materias primas que participan del mismo; esto da una idea clara de cómo la empresa llega a su producto final, identifica cuáles procesos 
impactan más en la facturación de energía eléctrica mensual, determina porcentajes de automatización en la planta analizando los distintos procesos, entre otras características. Identificar los procesos también permitirá desglosar el trabajo, de manera que sea más fácil de recopilar y analizar los resultados.

\subsubsection{Identificación de áreas}

Los distintos procesos realizados en la empresa son:

\section{Preparados de cilindros}

En este proceso se graban imágenes en varios cilindros de cobre, que luego serán insertados en las máquinas de rotograbado para la impresión de la imagen que el cliente desee para su producto.

\section{Preparado de tinta}

En este proceso se hacen las mezclas de tintas bases para conseguir el color deseado, en algunas ocasiones las tintas son mezcladas con oro o barniz para que se adhieran con más facilidad a algunos tipos de materiales de impresión.

\section{Rotograbado}

En este proceso, las máquinas de rotograbado imprimen a alta velocidad la imagen que el cliente desee que tenga su producto en el material que el mismo solicite.

\section{Laminado}

En este proceso se adhieren dos capas de materiales distintos de acuerdo con lo que requiera el cliente para su producto, se pueden realizar con distintos materiales de adhesión, como cera caliente, adhesivo con solvente o sin solvente. Los materiales que normalmente se unen son papel y plásticos de distintas naturalezas.

\section{Metalizado}

Este proceso es único y poco utilizado en la empresa, pero cuando se utiliza causa un consumo de energía muy significativo, por lo que se prefirió separar del proceso de laminado, aunque su objetivo es básicamente el mismo, unir una capa de algún metal a una capa de algún material plástico.

\section{Corte y acabado}

En este proceso los rollos de impresión entregados por el proceso de rotograbado, luego de pasar por el área de laminado o metalizado cuando así lo requieran, son cortados de acuerdo con los requerimientos del cliente (rollos o láminas).

\subsubsection{Flujogramas de procesos}

Como parte del conocimiento del procedimiento seguido para la elaboración del producto final de la empresa, se realizaron los flujogramas de los distintos procesos en la planta.
Las Figuras 4 a la 9 presentan los respectivos diagramas de flujo para cada proceso.

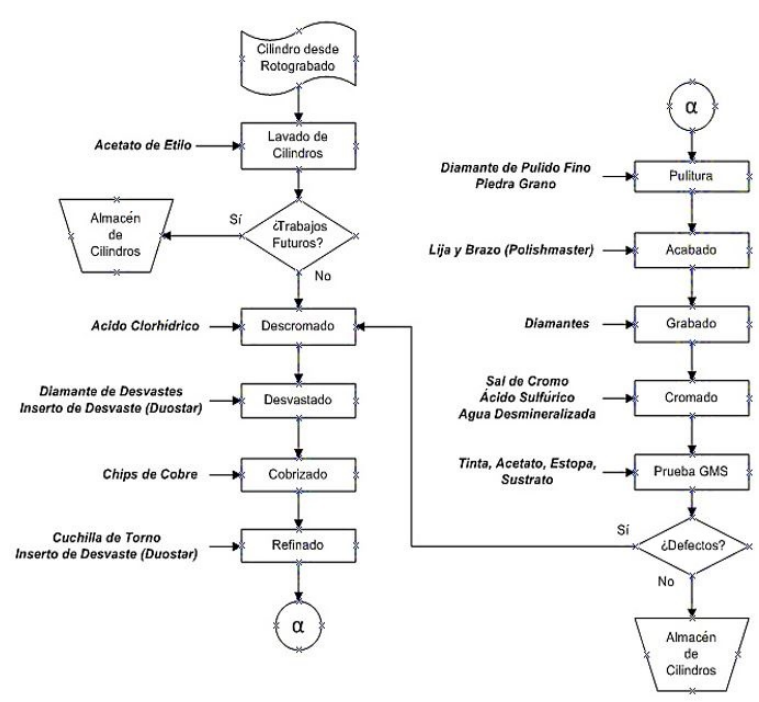

Figura 4. Flujograma de proceso del área de cilindros.
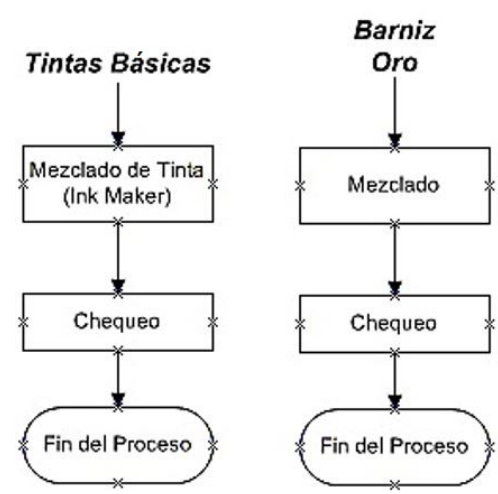

Figura 5. Flujograma de proceso del área de tintas.

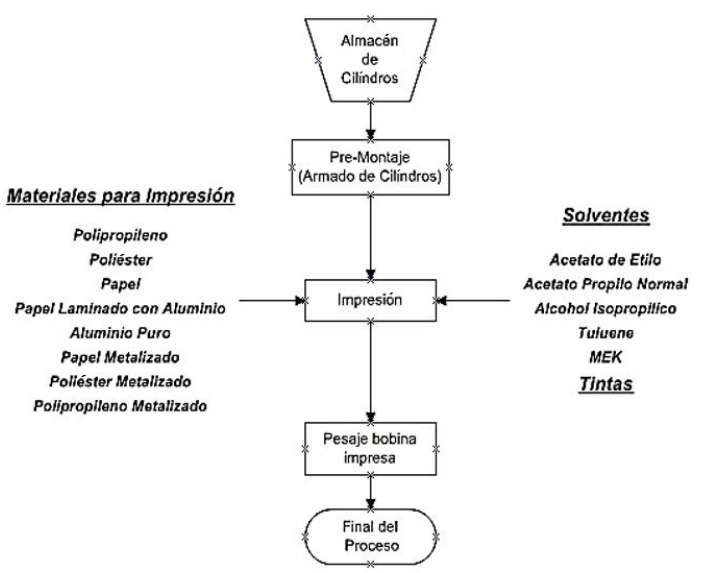

Figura 6. Flujograma de proceso del área de cilindros. 


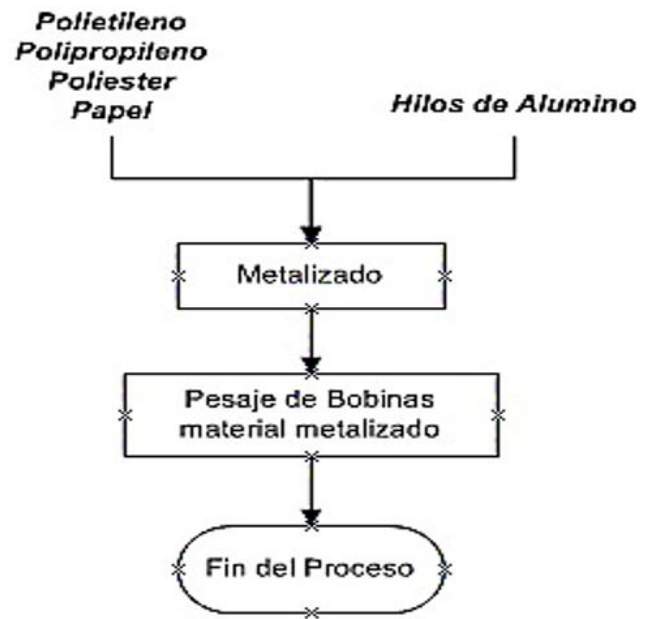

Figura 7. Flujograma de proceso del área de metalizado.

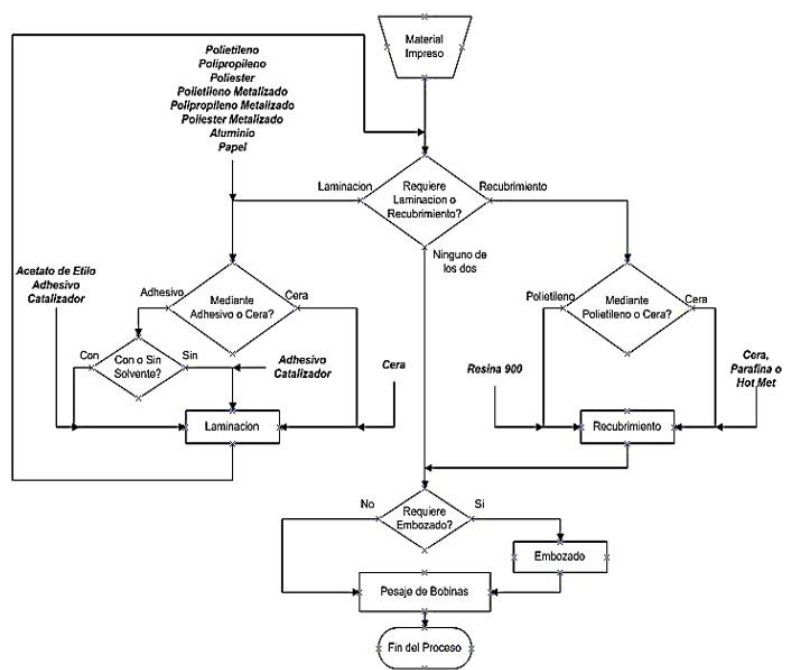

Figura 8. Flujograma del proceso de laminado.

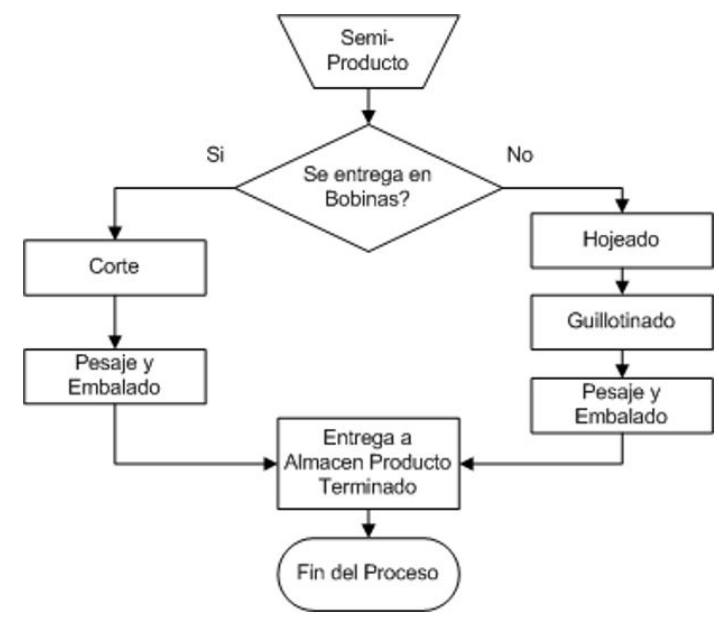

Figura 9. Flujograma del Proceso de Corte y Acabado.

\subsection{Censo de carga}

En el censo de carga se identificaron un total de 56 máquinas compuestas por motores y diversos elementos; y aproximadamente cincuenta motores independientes en las seis áreas descritas anteriormente, además de equipos de oficina en un total de 45 áreas de trabajo (oficinas, talleres, depósitos, etc.), para un total de 5946,35 kVA carga conectada.

La Tabla 1 presenta los motores independientes de mayor consumo en la planta.

Tabla 1. Estimación de carga de una unidad de enfriamiento, Chiller 2 - Westinghouse $-460 \mathrm{~V}$

\begin{tabular}{ccccc}
\hline Descripción & P (HP) & Vn (V) & In (A) & kVA \\
\hline Compresor & 20 & 460 & 32,5 & 25,89 \\
Compresor & 25 & 460 & 40 & 31,87 \\
Compresor & 20 & 460 & 32,5 & 25,89 \\
Compresor & 25 & 460 & 40 & 31,87 \\
Ventilador & 2 & 460 & 3,3 & 2,63 \\
Ventilador & 2 & 460 & 3,3 & 2,63 \\
Ventilador & 2 & 460 & 3,3 & 2,63 \\
Potencia aproximada de la máquina (kVA) & $\mathbf{1 2 3 , 4}$ \\
\hline
\end{tabular}

\subsection{Diagrama de Pareto y estratificación}

Finalizado el censo de carga de la planta y conocidos los datos de carga conectada en ella, se separan por cada proceso realizado en la planta las máquinas, equipos y motores involucrados en el mismo, para identificar los potenciales de ahorro. Los potenciales de ahorro se identificaron mediante el diagrama de Pareto.

La Figura 10 presenta el diagrama de Pareto de procesos generales, donde se presenta el porcentaje (\%) de carga de los procesos.

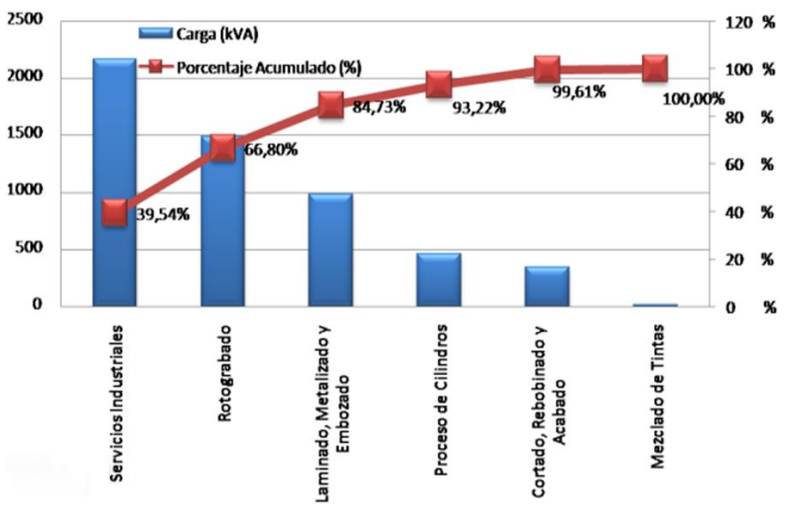

Figura 10. Diagrama de pareto de procesos generales.

Es de suma importancia identificar los elementos que pueden impactar notablemente en el consumo de energía general de la planta, ya que estos representan posibles puntos de ahorro, por lo tanto, en estos equipos se tomaron las mediciones de consumo de energía real. 
Los elementos de bajo consumo tienen poca importancia analizarlos, ya que cualquier medida o recomendación que se tome en ellos, no impactará significativamente en el ahorro energético total que se desea lograr.

A partir de la Figura 10, se puede identificar que existen tres procesos y un área que representan casi un $85 \%$ de la carga total de la planta: proceso de laminado metalizado y embobinado, rotograbado y el área de servicios industriales.

- Proceso de laminado metalizado y embobinado:

Dos equipos consumen en conjunto $15884 \mathrm{kWh}$ al mes, representando un $72 \%$ del consumo mensual estimado de este proceso. Uno de los equipos sobresale por su consumo debido a su operación continua durante el mes y el otro equipo por su elevado consumo puntual.

\section{- Rotograbado:}

Tres equipos que producen lo mismo, dos de ellos de igual tecnología, cuya producción es superior al tercer equipo. Los equipos de igual tecnología consumen en conjunto $105440 \mathrm{kWh}$ al mes, casi un $89 \%$ de la energía mensual total de este proceso, y el tercer equipo consume apenas $13830 \mathrm{kWh}$.

- Servicios industriales:

Los equipos que más energía consumen mensualmente son equipos de climatización y chillers; representan en conjunto $135000 \mathrm{kWh}$ al mes, casi un $45 \%$ del consumo mensual en esta área.

\subsection{Diagramas de control}

Con los datos de los últimos dos años de la facturación de energía eléctrica mensual, más los datos de producción mensual, se realizaron los gráficos que proporcionan información de la variación de consumo de energía basándose en la producción de la planta, consumos no asociados a la producción, puntos óptimos de producción, establecimientos de metas, entre otros.

\subsubsection{Gráfico de control}

Los gráficos de control son diagramas lineales que permiten observar el comportamiento de una variable en función de ciertos límites establecidos. Se usan como instrumento de autocontrol y resultan muy útiles como complemento a los diagramas causa y efecto, para detectar en cuales fases del proceso analizado se producen las alteraciones.

Este gráfico se usa para descartar los puntos ubicados fuera de los límites establecidos $( \pm 3 \sigma)$, ya que estos representan valores anormales $y$, de incluirse en este estudio, darían resultados incongruentes. Además, permite identificar los puntos que se acercan notablemente a los límites y encontrar las causas de esta desviación. Para realizar este gráfico se utilizaron los registros de pago de los últimos dos años a la empresa de servicio de energía eléctrica. La Figura 11 muestra el gráfico de control generado para la empresa. Ningún punto de consumo mensual sale de los límites establecidos, por lo que todos los puntos son válidos para este estudio.

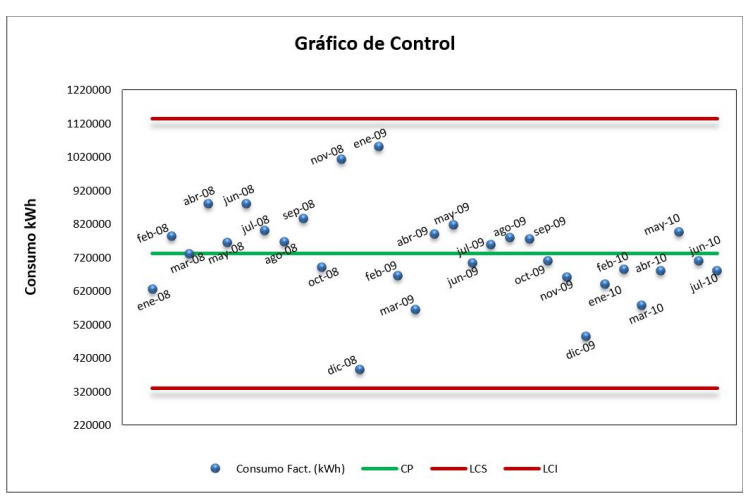

Figura 11. Gráfico de control.

\subsubsection{Gráfico energía producción vs. tiempo}

Este gráfico, presentado en la Figura 12, muestra simultáneamente el consumo energético y la producción en el tiempo, lo que permite observar la variación independiente de la energía eléctrica consumida $(\mathrm{kWh})$ y la cantidad de producción (t) a través del tiempo con el fin de identificar algún comportamiento anormal en algún periodo (mes) e identificar las razones que producen dicho comportamiento anormal [6].

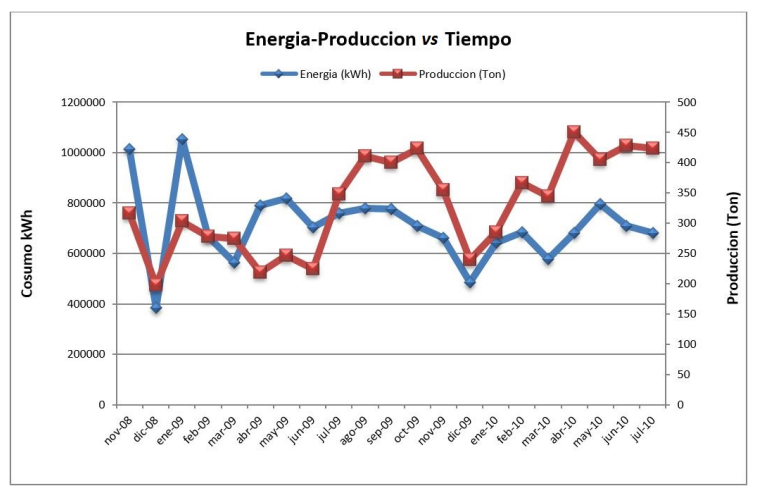

Figura 12. Gráfico de energía eléctrica - Producción vs. tiempo.

El gráfico energía-producción vs. tiempo de muestra que no existe un comportamiento uniforme desde el periodo noviembre 2008 a junio 2009, así como también la existencia de un cambio notable de la relación entre la producción y la energía eléctrica facturada a partir de julio 2009, debido a los cambios en el equipamiento 
de alto consumo, cuando la producción de la empresa se incrementó significativamente.

\subsubsection{Gráfico energía vs. producción.}

Este gráfico tiene como finalidad establecer la tendencia que existe entre la producción y el consumo energético, y de esta forma definir cuantitativamente el valor de la energía no asociada a la producción. Para las empresas industriales y de servicios, realizar un diagrama de dispersión de la energía usada en un tiempo determinado con respecto a la producción realizada o los servicios prestados durante ese mismo periodo, revela importante información sobre el proceso, ya que al realizar la recta de caracterización del consumo vs. producción, se puede establecer el consumo energético no asociado a la producción [6].

En el caso de la empresa en estudio, como muestra la Figura 12 se presentaron dos tendencias de consumo de energía muy distintas, debido a dos motivos fundamentales: el primero es la incorporación de máquinas de mayor eficiencia y el segundo es la normativa publicada en noviembre del 2009 en la Gaceta Oficial de la República Bolivariana de Venezuela Número 39.298 [7], ordenando reducir en un $20 \%$ el consumo de energía eléctrica, para no ser penalizado con un corte en el suministro de electricidad.

Por este motivo se decidió realizar gráficos individuales para cada una de las dos tendencias, uno para el periodo entre noviembre 2008 (fecha a partir de la cual se posee datos de producción) y agosto 2009, Figura 13, y otro para los meses desde septiembre 2009 hasta julio 2010, Figura 14.

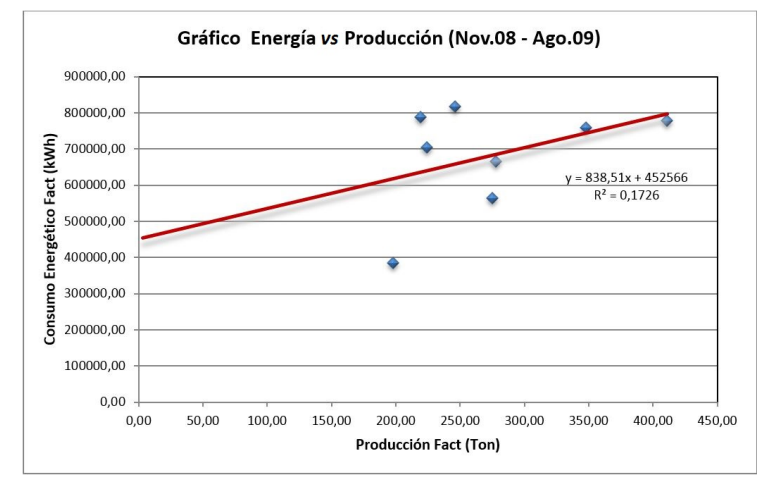

Figura 13. Gráfico de energía vs. producción (Nov. $08-$ Agos. 09).

Antes de septiembre 2009, Figura 13, el consumo no asociado a la producción era de $452566 \mathrm{kWh}$, después de septiembre 2009, Figura 14, el consumo no asociado a la producción era de 300054 kWh $(66 \%$ de lo que era antes), esto es debido a la orden de reducción de consumo, ya que la empresa adoptó medidas de ahorro energético, tales como reemplazo de luminarias, apagado de circuitos de iluminación, apagado de presurizadores de gran tamaño, adquisición de un compresor de aire más eficiente, entre otras.

La Figura 13 muestra una correlación entre los puntos muy baja, por diversos motivos que solo la empresa conoce (información confidencial), no existe una proporcionalidad entre lo que se produce y lo que se consume. También es de destacar que noviembre 2008 y enero 2009, fueron los dos meses de mayor consumo en la planta y no se encontró una causa concreta para dicho incremento, ya que la producción de este periodo no justifica este hecho; es importante resaltar que estos dos puntos disminuyen aún más la correlación existente entre los datos graficados, lo que indica la existencia de algún evento irregular en planta durante esos meses, o un error en la medición-estimación hecha por la empresa de servicio de energía eléctrica.

La Figura 14 muestra una correlación de un $57 \%$ entre los datos graficados, lo que indica que existe una mayor fiabilidad en cuanto a estos, por lo que el estudio del consumo energético se hará tomando como base este periodo (septiembre 2009-julio 2010).

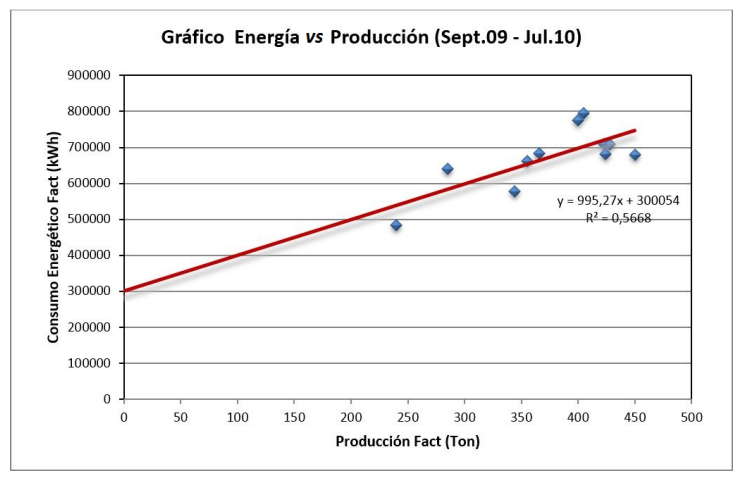

Figura 14. Gráfico de energía vs. producción (sept. 09 jul. 10).

\subsubsection{Establecimiento del gráfico meta vs. pro- ducción.}

Una vez realizado los gráficos de energía vs. producción, se establecen las metas de consumo.

Para establecer las metas y que estas sean alcanzables, inicialmente se seleccionaron los meses de mejor eficiencia de trabajo de la planta, es decir, aquellos que se encuentran por debajo de la recta trazada en la Figura $14 \mathrm{y}$, mediante estos puntos seleccionados, se establece una nueva tendencia de consumo, la cual se define como la tendencia meta, mostrado en la Figura 15.

En la Figura 15 la tendencia meta establecida es paralela a la tendencia actual, pero desplazada verticalmente hacia abajo. Esta nueva tendencia indica cuál debería ser el consumo de energía eléctrica para cada valor de producción, para que la planta trabaje de forma más eficiente. El consumo no asociado a 
la producción de la tendencia meta es de 213871 $\mathrm{kWh}$, mientras que el de la tendencia actual es de $300054 \mathrm{kWh}$, esto implica que si la planta logra mantener su producción dentro de la tendencia meta, se alcanzará un $29 \%$ de ahorro de energía.

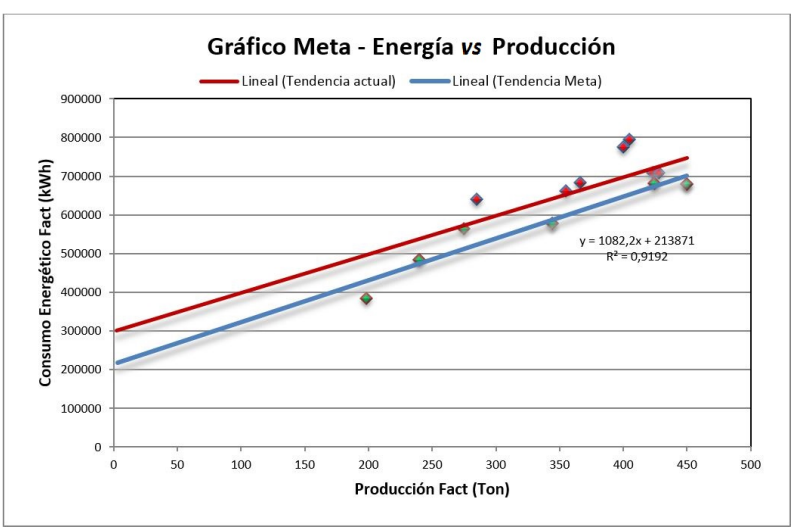

Figura 15. Gráfico meta - energía vs. producción.

Cabe destacar que la meta establecida anteriormente se fijó en función de valores reales que la planta ya ha alcanzado, por lo que si la planta trabaja implantando nuevos sistemas de ahorros, los resultados pueden ser aún más eficientes.

\subsubsection{Gráfico de índice de consumo vs. produc- ción.}

Con los valores de la tendencia de consumo actual y la tendencia de consumo meta, y dividiendo estas entre la producción, se puede obtener el gráfico de índice de consumo vs. producción, mostrado en la Figura 16, que indica la energía necesaria para producir una tonelada para un determinado valor de producción.

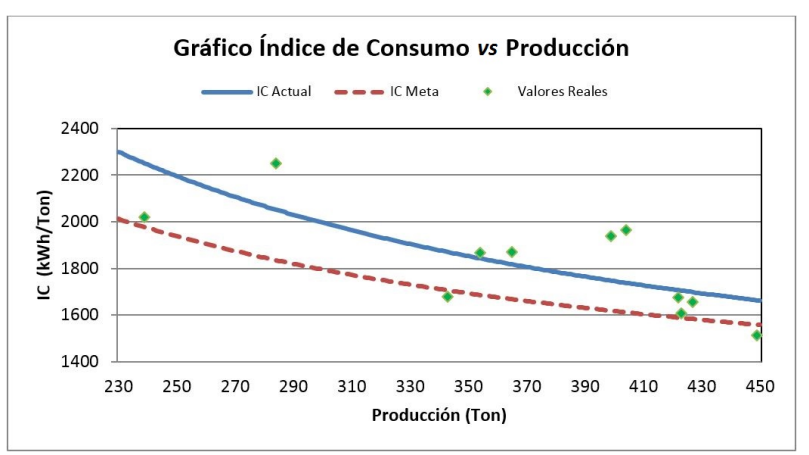

Figura 16. Gráfico índice de consumo vs. producción.

Como se puede observar de la Figura 16, mientras mayor es la producción en la planta, menor es el impacto de la energía eléctrica en el costo de una tonelada de producción, debido a esto es importante que la producción en la empresa se mantenga a un nivel elevado, con el fin de reducir la cantidad de $\mathrm{kWh}$ por tonelada producida. Además, se pueden observar dos curvas de índice de consumo teórico, una para la tendencia de consumo actual y otra para la tendencia de consumo meta.

El análisis del índice de consumo, resumido en la Tabla 2, indica que además de la importancia de mantener la producción elevada, es necesario cumplir las metas establecidas para reducir notablemente el impacto de la energía por tonelada de producto.

Tabla 2. Análisis del gráfico de índice de consumo

\begin{tabular}{ccc}
\hline Toneladas & $\begin{array}{c}\text { IC actual } \\
\mathbf{k W h} / \mathbf{t}\end{array}$ & $\begin{array}{c}\text { IC meta } \\
\mathbf{k W h} / \mathbf{t}\end{array}$ \\
\hline 250 & 2196,0 & 1937,0 \\
350 & 1852,0 & 1693,0 \\
450 & 1662,0 & 1557,0 \\
\hline
\end{tabular}

\subsubsection{Medidas propuestas sobre la base del gráfico meta de energía vs. producción.}

Una vez cuantificado el valor de energía eléctrica (kWh) que se puede ahorrar mensualmente mediante la aplicación de las medidas propuestas, cambio de luminarias, apagado de luminarias en horas de descanso, reducción $20 \%$ iluminación planta y exterior, cambio de motores, apagado de aires acondicionado y de equipos de oficina durante las horas de descanso y el utilizar el compresor nuevo $100 \%$ del tiempo; la tendencia de consumo meta establecida sufrirá una modificación, ya que este gráfico ha sido formado inicialmente por los mejores valores realmente alcanzados en la operación en la planta.

A continuación, se establecen dos nuevos gráficos meta, uno considerando solo las medidas que no requieran inversión monetaria en maquinarias y equipos, y otro que incluye todas las propuestas de ahorro energético, para sentar las bases del análisis económico, ambos casos darán como resultado una tendencia paralela a la meta anterior, pero desplazada hacia abajo, esto es, con un consumo no asociado a la producción menor.

En el establecimiento de los nuevos gráficos metas se aplicaron factores a los valores de ahorro energético por concepto de iluminación, equipos de oficinas y de aire acondicionado con el fin de darle más realismo a los resultados, ya que en algunas áreas ya se aplican las medidas de apagar la iluminación y los computadores en horas de descanso, y los equipos de climatización no trabajan al $100 \%$ de su capacidad durante todo su periodo de trabajo. Estos factores son los siguientes:

1) Se aplicó un factor de 0,6 al valor obtenido por concepto de ahorro al apagar las luminarias.

2) Se aplicó un factor de 0,8 al valor obtenido por concepto de ahorro al apagar los equipos de oficinas. 
3) Se utilizó un factor de 0,9 al consumo nominal de aires condicionados.

Estos factores fueron definidos mediante la observación de las costumbres del personal de la empresa; las Tablas 3 y 4 resumen los valores de energía mensual ahorrada $(\mathrm{kWh})$ debido a cada propuesta.

Tabla 3. Análisis del gráfico de índice de consumo

\begin{tabular}{cc}
\hline Propuesta & $\begin{array}{c}\text { Ahorro mensual } \\
\mathbf{k W h}\end{array}$ \\
\hline $\begin{array}{c}\text { Apagado de luminarias } \\
\text { durante horas de descanso }\end{array}$ & 1992,42 \\
$\begin{array}{c}\text { Reducción 20 \% iluminación } \\
\text { planta y exterior }\end{array}$ & 6163,20 \\
Apagado de A/A durante & 4303,28 \\
$\quad$ horas de descanso & 155,20 \\
$\begin{array}{c}\text { Apagado de equipos de oficina } \\
\text { durante horas de descanso } \\
\text { Utilización del compresor } \\
\text { nuevo 100 \% del tiempo } \\
\text { TOTAL }\end{array}$ & 7148,00 \\
\hline
\end{tabular}

Tabla 4. Ahorro mensual de energía eléctrica para todas las propuestas

\begin{tabular}{|c|c|}
\hline Propuesta & $\begin{array}{c}\text { Ahorro mensual } \\
\mathrm{kWh}\end{array}$ \\
\hline $\begin{array}{l}\text { Cambio luminarias de } \\
4 \times 40 \mathrm{~W} \text { a } 3 \times 32 \mathrm{~W}\end{array}$ & 2647,04 \\
\hline $\begin{array}{c}\text { Apagado de luminarias } \\
\text { durante horas de descanso }\end{array}$ & 1765,40 \\
\hline $\begin{array}{c}\text { Reducción } 20 \% \text { iluminación } \\
\text { planta y exterior }\end{array}$ & 6163,20 \\
\hline $\begin{array}{l}\text { Cambio de motores } \\
\text { (disminuir pérdidas) }\end{array}$ & 30778,73 \\
\hline $\begin{array}{c}\text { Apagado de A/A } \\
\text { durante horas de descanso }\end{array}$ & 4303,28 \\
\hline $\begin{array}{l}\text { Apagado de equipos de oficina } \\
\text { durante horas de descanso }\end{array}$ & 155,20 \\
\hline $\begin{array}{l}\text { Utilización del compresor nuevo } \\
100 \% \text { del tiempo }\end{array}$ & 7148,00 \\
\hline TOTAL & 52960,85 \\
\hline
\end{tabular}

Las Figuras 17 y 18 muestran respectivamente los nuevos gráficos meta de energía vs. producción respectivamente para las medidas sin inversión y para todas las medidas propuestas.

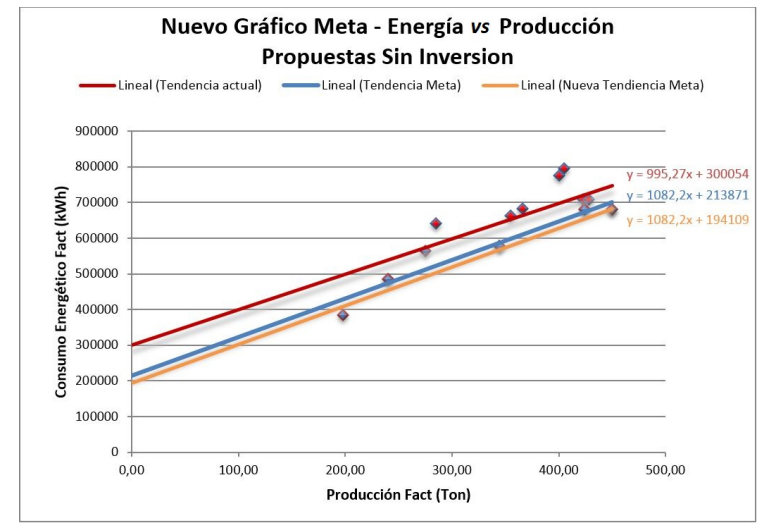

Figura 17. Nuevo gráfico meta - energía vs. producción propuestas sin inversión.

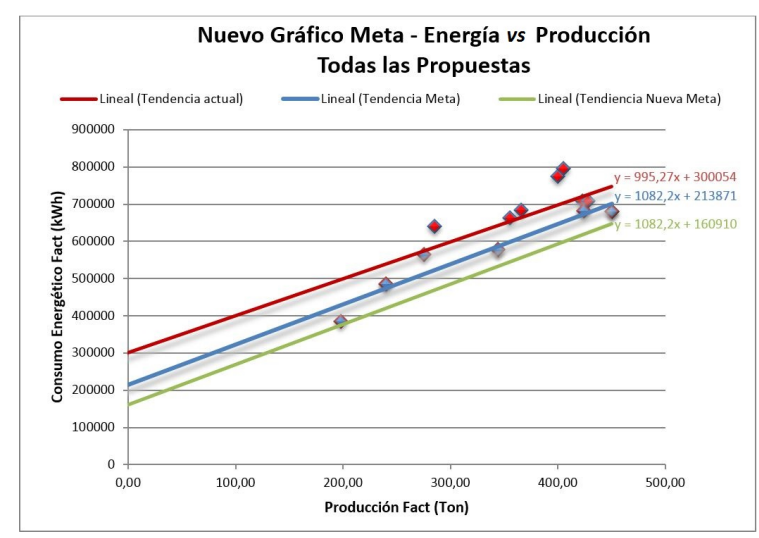

Figura 18. Nuevo gráfico meta - energía vs. producción todas las propuestas.

Las Figuras 17 y 18 muestran en rojo la tendencia actual de la planta, cuyo consumo no asociado a la producción es de $300054 \mathrm{kWh}$ al mes, y en color azul la tendencia meta establecida para la planta sin incluir cambios, cuyo valor no asociado a la producción es de $213871 \mathrm{kWh}$ al mes.

La Figura 17 muestra en naranja la nueva tendencia meta establecida sobre base de la implementación de las medidas de ahorro sin inversión, cuyo valor asociado a la no producción es de $194109 \mathrm{kWh}$ al mes, esto representa un $35 \%$ respecto a la tendencia actual y un $9 \%$ respecto a la tendencia meta establecida, Figura 8.

La Figura 18 muestra en verde la nueva tendencia meta establecida de acuerdo con la implementación de todas las medidas propuestas, que arroja un valor de consumo no asociado a la producción de 160 910,00 $\mathrm{kWh}$ al mes, lo que representa un $46 \%$ del consumo actual no asociado a la producción y un $25 \%$ de ahorro adicional con respecto a la meta previamente establecida.

A modo de comparación la Tabla 5 muestra la cantidad de residencias que se pudiera alimentar con el ahorro energético obtenido al implantar las medidas 
propuestas en la empresa Montana Gráfica filial del Grupo Corimon Pintura C. A., tomando como consumo promedio mensual de una vivienda $500 \mathrm{kWh}$ mensuales, tarifa mínima para servicio residencial general, tipificada según esquema tarifario como Tarifa 02: Servicio residencial general, presente en el Artículo 9 punto 9.2, publicado el 3 de abril de 2002 en la Gaceta Oficial de la República Bolivariana de Venezuela Número 37.415 [8], considerada la tarifa legal. Se pueden alimentar 40 casas poniendo en marcha las propuestas sin inversión monetaria en equipos y maquinarias, 106 casas si se consideran todas las propuestas de ahorro energético y, en un caso ideal que la empresa logre operar en los puntos óptimos de consumo de acuerdo con la producción además de la implementación de todas las acciones que se proponen en este trabajo, se llegaría a alimentar hasta 278 residencias con la energía que se deja de desperdiciar.

Tabla 5. Cantidad de viviendas sociales que pudiesen ser alimentadas con la energía ahorrada

\begin{tabular}{ccc}
\hline Acciones a implantar & $\begin{array}{c}\text { Energía ahorrada } \\
\text { al mes (kWh) }\end{array}$ & $\begin{array}{c}\text { N. }{ }^{\circ} \text { de } \\
\text { casas }\end{array}$ \\
\hline Propuestas sin inversión & 19762,10 & 40 \\
Todas las propuestas & 52960,85 & 106 \\
$\quad$ Nueva meta & 139144,00 & 278 \\
(todas las propuestas) & & \\
\hline
\end{tabular}

\section{Seguimiento y control}

En esta sección se procede a realizar una evaluación del proyecto en función de los resultados obtenidos debido a la implementación de las medidas de ahorro propuestas, además de que la empresa pueda mantenerse en los puntos óptimos de consumo meta establecidos.

El Ejecutivo implantó en 2009 una política de reducción en los consumos debido a crisis energética nacional. La Gerencia del Grupo Corimon, de la cual forma parte la empresa Montana Gráfica, desde la fecha implantó una serie de medidas, Figura 19, para disminuir el consumo de energía en las diferentes plantas que lo integran.

Se adquirieron grupos electrógenos para suplir energía a los procesos críticos durante interrupciones o racionamiento.

Se solventaron situaciones como motores subcargados, desequilibrios en la red de alimentación, operación a tensión reducida y contenido armónico introducido por las cargas no lineales que impactan en el consumo energético.

Se realizó un estudio de la operación de las máquinas más importantes en los procesos que de acuerdo con el diagrama de Pareto presentaba el mayor consumo.
Los ahorros energéticos obtenidos oscilan entre un $18 \%$ y un $26 \%$ de acuerdo con las condiciones, las políticas de la empresa y el nivel de compromiso alcanzado por el personal.

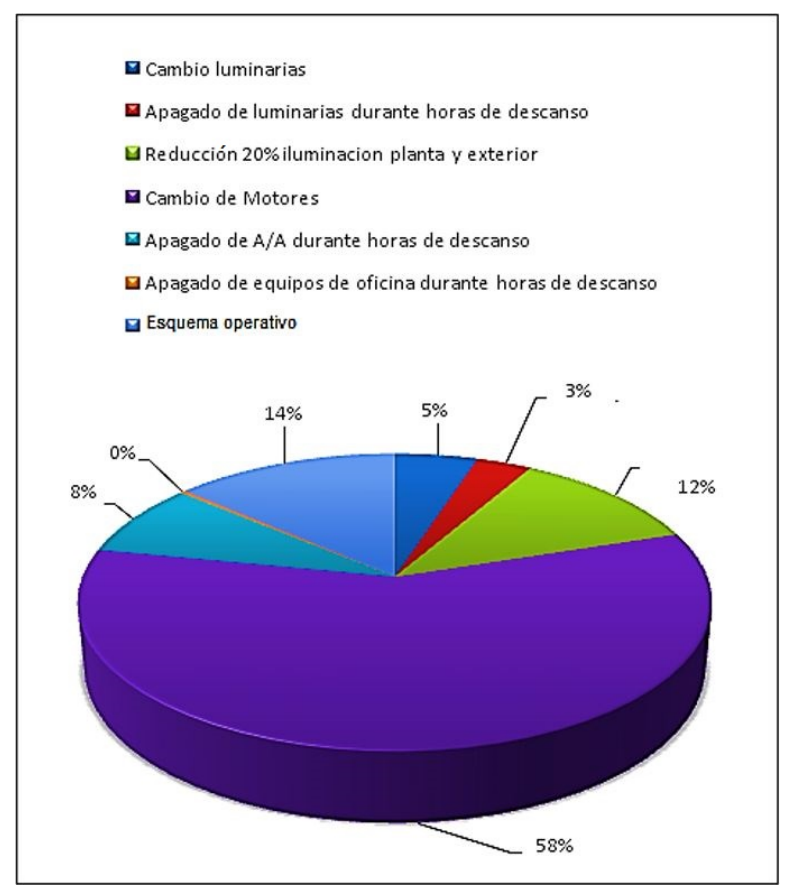

Figura 19. Promedio de porcentaje de distribución de ahorro de las medidas propuestas.

De la caracterización constante de la empresa se intuye la posibilidad de mejorar las metas de ahorro energético a medida que se analice e implanten nuevas medidas.

La factibilidad económica del proyecto se obtuvo sobre la base que la empresa opere en condiciones consideradas óptimas de consumo vs. producción (tendencia meta), además de las medidas propuestas sin inversión durante un periodo de 7 años (medidas a corto y mediano plazo). La tasa mínima de rendimiento se consideró constante en $17 \%$ durante el periodo de estudio.

Para el primer año $(\mathrm{t}=0)$ se estimó únicamente el costo del proyecto como flujo monetario, para los demás años se considera como flujo neto el ahorro anual que se obtuvo mediante las propuestas sin inversión y operación de la planta bajo la tendencia meta consumo vs. producción, pero incrementándose conforme las medidas fueron implantadas. Se considera la tarifa eléctrica constante bajo todo el periodo de estudio.

Los ingresos superan los costos, Tabla 6, por lo que económicamente el beneficio es superior al mínimo exigido de acuerdo con el análisis del proyecto, en 7 años (2009-2016). 
Tabla 6. Interpretación del valor del proyecto 2009-2016

\begin{tabular}{cccccc}
\hline Año & $\begin{array}{c}\text { Inversión por } \\
\text { recuperar al } \\
\text { inicio }\end{array}$ & $\begin{array}{c}\text { Flujo } \\
\text { neto }\end{array}$ & $\begin{array}{c}\text { Rendimiento } \\
\text { mínimo }\end{array}$ & $\begin{array}{c}\text { Inversión } \\
\text { recuperada }\end{array}$ & $\begin{array}{c}\text { Inversión por } \\
\text { recuperar }\end{array}$ \\
\hline 1 & 98104,00 & 12230,25 & 16677,68 & $-4447,43$ & 102551,43 \\
2 & 102551,43 & 24460,50 & 17433,74 & 7026,76 & 95524,67 \\
3 & 95524,67 & 36690,75 & 16239,19 & 20451,56 & 75073,12 \\
4 & 75073,12 & 48921,24 & 12762,43 & 36158,81 & 38914,31 \\
5 & 38914,31 & 48921,24 & 6615,43 & 42305,81 & $-3391,5$ \\
6 & 30582,87 & 65228,64 & 5199,08 & 74797,60 & $-1757,98$ \\
7 & 15852,69 & 65228,64 & 2694,95 & 87513,19 & $-153,21$ \\
8 & 12458,67 & 86971,94 & 2117,96 & 154725,23 & $-79,41$ \\
\hline
\end{tabular}

\section{Conclusiones}

En este trabajo se presenta una metodología para cuantificar el ahorro de energía posible en una industria, mediante un procedimiento basado en los gráficos de control que permite sistematizar el cálculo de los ahorros de energía posibles, lo que es una manera de ayudar a solucionar el problema energético actual.

El estudio sistemático de los posibles ahorros basado en el método, permite mejorar los resultados obtenidos anteriormente con cambios no sistemáticos.

Las propuestas realizadas según el método presentado lograron un ahorro, cada mes al sistema de generación de electricidad de un total de 138 024,84 kWh por mes, en promedio. Y, la empresa logró operar en puntos óptimos de consumo de acuerdo con la producción, además de la implementación de acciones, el ahorro es equivalente a la energía eléctrica necesaria para alimentar hasta 278 residencias con la energía que se deja de desperdiciar. La aplicación generalizada de la metodología propuesta permitiría reducir el problema energético nacional de una manera sistemática, eliminando el derroche de energía sin afectar el nivel de la producción industrial.

\section{Agradecimiento}

A las personas que sin su valiosa colaboración no hubiese sido posible este trabajo:

A Daniel Manosalva y Aron Abramof, ingenieros electricistas.

A los profesores José Aller y Gleb Machado del Departamento de Conversión y Transporte de Energía de la Universidad Simón Bolívar.

A la profesora Milagros Peña del Departamento de Ingeniería Eléctrica de la Universidad de Carabobo. Así como a la empresa Montana Gráfica, filial del Grupo Corimon Pintura C. A.

\section{Referencias}

[1] Colectivo de autores, Gestión Energética Empresarial, Centro de Estudios de Energía y Medio Ambiente, Ed. Universidad de Cienfuegos, 2001. [Online]. Available: https://goo.gl/UPpCnv

[2] Comisión Eléctrica Nacional Colegio de Ingenieros de Venezuela (CIV). (2017). [Online]. Available: https://goo.gl/WbVNFm

[3] D. Manosalva and A. Abramoff, "Implantación de un sistema integral de la energía en la Empresa Montana Gráfica, filial del Grupo Corimon C. A." in Tesis de grado. Facultad de Ingeniería, Escuela de Ingeniería Eléctrica, Departamento de Potencia, Universidad de Carabobo, Valencia, Estado Carabobo, Venezuela, 2011.

[4] J. Aller, L. Salazar, A. Bueno, and M. Peña, "Metodología para la implantación de un sistema integral de gestión de la energía," in IX Congreso de Instalaciones Eléctricas: "Hacia la eficiencia en los servicios de electricidad y telecomunicaciones", 2010, pp. 1-8. [Online]. Available: https://goo.gl/UPo6pa

[5] ISO 50001:2011. Energy management systems Requirements with guidance for use, International Organization for Standardization Std., 2011. [Online]. Available: https://goo.gl/XLWo5s

[6] A. Borroto and J. Monteagudo, "Gestión energética en el sector productivo y los servicios," in Universidad de Cienfuegos, Cuba, pp. 20-22, 2006.

[7] "Gaceta oficial de la República Bolivariana de Venezuela, año CXXX, mes I, Caracas Artículo 1, p. 2." 3 de noviembre de 2009.

[8] "Gaceta oficial de la República Bolivariana de Venezuela, año CXXIX, mes VI, Caracas, Artículo 9," 3 de abril de 2002. 


\title{
FACTORES PARA SELECCIONAR TUBERÍAS DE CONDUCCIÓN DE GAS LICUADO DE PETRÓLEO EN EL ECUADOR
}

\author{
Factors for selecting Petroleum \\ Liquefied Gas CONDUCTION PIPES IN \\ ECUADOR
}

\author{
Diego Venegas ${ }^{1, *}$, César Ayabaca ${ }^{2}$, Santiago Celi $^{3}$, Juan Rocha $^{3}$, Euro Mena $^{4}$
}

\section{Resumen}

Para realizar instalaciones residenciales, comerciales e industriales de gas licuado de petróleo (GLP) en el Ecuador, rige la norma técnica ecuatoriana INEN 2260:2010, la cual establece los lineamientos mínimos de seguridad que se deben tener en cuenta en este tipo de instalaciones. Para conducir este hidrocarburo, la norma acepta varios materiales de tuberías, métodos de unión con accesorios, formas de instalación, lo que hace que el responsable del dimensionamiento y planeación de este tipo de sistemas tenga varias opciones para escoger al momento de encaminar un proyecto determinado. El presente trabajo muestra algunos factores que inciden en la selección de la tubería más adecuada para sistemas de GLP, cumpliendo con la norma técnica obligatoria en el Ecuador. Se compara la resistencia a la tracción, dureza y peso como propiedades de los materiales aceptados; tiempos de ejecución de uniones entre tuberías y accesorios y, finalmente, se comparan los costos asociados a los materiales, mano de obra y mantenimiento que debe darse a estos sistemas.

Palabras clave: materiales recomendados, norma técnica, tuberías, mejor aplicación, propiedades.

\begin{abstract}
In order to build residential, commercial and industrial installations of Liquefied Petroleum Gas (LPG) in Ecuador, the INEN 2260: 2010 Ecuadorian Technical Regulation establishes the minimum safety guidelines that must be taken into account in this type of installations. To conduct this hydrocarbon, the Standard accepts several materials of pipes, methods of union with accessories, forms of installation, which makes the responsible for the sizing and planning of this type of systems have several options to choose from when directing a determined project. This paper shows some factors that affect the selection of the most suitable pipeline for LPG systems, complying with the mandatory Technical Standard in Ecuador. The mechanical strength, hardness and weight are compared as properties of the accepted materials; the execution times of pipes and fittings and, finally, the costs associated with materials, labor and maintenance that must be given to these systems are compared.
\end{abstract}

Keywords: Recommended materials, Technical Standard, pipes, best application, properties.

\footnotetext{
1,* Facultad de Ingeniería Universidad de Concepción, Concepción - Chile.

Autor para correspondencia : diegofvenegas@udec.cl. (1) http://orcid.org/0000-0002-7376-6272

${ }^{2}$ Departamento de Ingeniería Mecánica, Escuela Politécnica Nacional, Quito - Ecuador. (D) http://orcid.org/0000-0002-3728-7514

${ }^{3}$ Facultad de Arquitectura e Ingeniería, Universidad Internacional SEK, Quito - Ecuador.

(D) http://orcid.org/0000-0002-8302-3413, (D) http://orcid.org/0000-0003-0660-7199

${ }^{4}$ Departamento de Energía y Mecánica, Universidad de las Fuerzas Armadas, Latacunga - Ecuador.

(D) http://orcid.org/0000-0001-7872-0292

Recibido: 27-06-2017, aprobado tras revisión: 12-08-2017

Forma sugerida de citación: Venegas, D.; Ayabaca, C.; Celi, S.; Rocha, J.; Mena, E. (2018). «Factores para seleccionar tuberías de conducción de gas licuado de petróleo en el Ecuador». INGENIUS. N. ${ }^{\circ} 19$, (enero-junio). pp. 51-59. DOI: https://doi.org/10.17163/ings.n19.2018.05.
} 


\section{Introducción}

El gas licuado de petróleo (GLP) es un combustible utilizado para aplicaciones residenciales, comerciales e industriales [1,2], y en el Ecuador el consumo de este hidrocarburo es muy frecuente, por lo que para el sector residencial el Gobierno nacional ha previsto un precio subsidiado $[3,4]$, ya que brinda varias ventajas entre las que se pueden mencionar [5-8]:

- Alto poder calorífico comparado con otras fuentes de energía.

- Limpio en términos de emisiones de gases contaminantes.

- Satisface varias necesidades energéticas.

Siendo un combustible muy explosivo e inflamable [9], se requiere mucha precaución al momento de planificar, dimensionar y seleccionar sus sistemas de almacenamiento (recipientes), de transporte (tuberías), equipos de consumo y elementos de protección y seguridad [10].

El riesgo en un sistema de GLP siempre está presente y no se lo puede eliminar [11], pero se lo puede minimizar si los sistemas que lo contienen son concebidos técnicamente $[12,13]$, de acuerdo con los lineamientos establecidos en normas técnicas [14] donde se indican los requerimientos mínimos de seguridad al momento de realizar las instalaciones.

En el Ecuador se encuentra vigente la norma INEN 2260:2010 «Instalaciones de gases combustibles para uso residencial, comercial e industrial. Requisitos» [15], en la cual se establecen los parámetros mínimos de seguridad obligatorios en el territorio nacional, y abarca todos los componentes necesarios en una instalación, desde los tanques de almacenamiento, tuberías, sistemas de regulación y control, sistemas de prevención, mantenimiento, entre otros.

En la naturaleza el GLP se encuentra en estado gaseoso, pero para facilitar su almacenamiento en recipientes se lo lleva a estado líquido aumentando su presión y bajando su temperatura [16]. Al interior de estos recipientes se produce un fenómeno de vaporización natural del líquido dado por el intercambio de calor entre este y el medioambiente [17], y en este estado es transportado por tuberías para su consumo.

Estas tuberías como mínimo deben cumplir con lo siguiente $[18,19]$ :

- Los materiales de fabricación deben ser compatibles con el combustible que están transportando.

- Su dimensión debe ser la adecuada para conducir el caudal que se requiere en la operación de los equipos de consumo.

- Deben soportar la presión de prueba y la presión de servicio a las que serán sometidas.
- Deben soportar las condiciones físicas del entorno donde serán instaladas.

Los tipos de materiales de tuberías que la norma técnica acepta para la instalación de los sistemas de GLP se muestran en la Tabla 1.

Tabla 1. Estimación de carga de una unidad de enfriamiento, Chiller 2 - Westinghouse $-460 \mathrm{~V}$

\begin{tabular}{cc}
\hline Tubería & Norma de fabricación \\
\hline \multirow{2}{*}{ Acero al carbono } & ASTM A 53 $[20]$ \\
& ISO 65 (serie Heavy) $[21]$ \\
Acero inoxidable & ANSI/AGALC1 $[22]$ \\
& ASTM A 240 [23] \\
Cobre & ISO 1640 [24] \\
& ASTM B88 [25] \\
Polietileno & ISO 4437 [26] \\
& ASTM D2513 [27] \\
P-Al-P* & AS-4176 [28] \\
& ISO 17484-1 [29] \\
\hline * P-Al-P Polietileno aluminio polietileno.
\end{tabular}

Se puede apreciar que la naturaleza de los materiales aceptados puede ser metálica o plástica, y para cada uno de ellos existe un procedimiento o método de unión de tuberías con sus accesorios. En la Tabla 2 se muestran aquellos procedimientos más comúnmente usados para unir tuberías con sus accesorios.

Tabla 2. Métodos de unión de tubería con accesorios [30]

\begin{tabular}{cc}
\hline Tubería & Procedimiento de unión \\
\hline Acero al carbono & SMAW o roscado \\
Acero inoxidable & Pressing fit \\
Cobre & Oxiacetilénico \\
Polietileno & Termofusión o electrofusión \\
P-Al-P & Termofusión o electrofusión \\
\hline
\end{tabular}

Por facilidad de inspección, mantenimiento y reparación en caso de fugas, se recomienda que las tuberías que conducen GLP sean instaladas vistas, sin embargo, por cuestión de esteticidad, los usuarios prefieren que estén ocultas.

La norma técnica acepta que las tuberías para conducción de GLP puedan ocultarse si se las instala:

- al interior de ductos,

- encamisadas en una tubería de mayor resistencia mecánica,

- embebidas en paredes y pisos, siempre y cuando sean recubiertas con un material de fácil remoción o,

- enterradas si se les brinda la debida protección contra daños físicos o contra la corrosión. 
Tabla 3. Formas de instalación de tubería [31]

\begin{tabular}{cccccc}
\hline $\begin{array}{l}\text { Forma de } \\
\text { instalación }\end{array}$ & Acero & $\begin{array}{l}\text { Acero } \\
\text { inoxidable }\end{array}$ & Cobre & Polietileno & P-Al-P \\
\hline Vista & Sí & Sí & Sí & No & No \\
Embebida & Sí & Sí & Sí & No & Sí \\
En ducto & Sí & Sí & Sí & No & Sí \\
Enterrada & Sí & Sí & Sí & Sí & Sí \\
Empotrada* & No & No & No & No & No \\
\hline *Empotrada: Aquella tubería que es fundida en la edificación pasando \\
\multicolumn{7}{l}{}
\end{tabular}

En la Tabla 3 se indican las formas aceptadas para instalación de tuberías de acuerdo con el material empleado.

Con estas opciones de materiales de tuberías, procedimientos de unión con accesorios y formas de instalarlas, hay varios factores adicionales que pueden incidir en la selección adecuada para una instalación de GLP en el Ecuador. En este trabajo se presentan varios de estos factores que pueden influir para obtener una selección más acorde a las características propias de cada usuario, y de las condiciones particulares que las rodea.

\section{Materiales y métodos}

\subsection{Propiedades del material}

Se compararon las siguientes propiedades físicas y mecánicas de los materiales indicados en la Tabla 1: resistencia a la tracción, dureza y peso por metro de tubería.

- Para medir la resistencia mecánica se utilizó una máquina de ensayos universal Tinius Olsen modelo Súper L-20, serie 80700-1. El criterio para la medición está basado en el ASTM E-8M [32].

- El durómetro Rockwell empleado para medir la dureza es un Mitutoyo, modelo Durotwin, serie BG000062, penetrador de diamante para escalas A-D-C, penetrador bola de acero $1 / 16$ " para escalas F-B-G, con lectura analógica. Para validar el procedimiento de medición de dureza se ha empleado la norma ASTM E18 03 método estándar para dureza Rockwell por indentación [33] y ASTM D785 método de prueba estándar para la dureza Rockwell de plásticos y materiales aislantes eléctricos [34].

- Para medir el peso de tuberías se utilizó la balanza Shimadzu Unibloc Cap. 220 g.

\subsection{Tiempos de instalación}

Cada uno de los procedimientos descritos en la Tabla 2 tiene sus particularidades, su forma de ejecución, su complejidad y por tanto, su tiempo de instalación.
Se procedieron a realizar uniones en tubería de 1" y bajo los procedimientos establecidos en la Tabla 2, todos ellos con personal calificado con vasta experiencia en el montaje de sistemas de GLP.

- El primer procedimiento consistió en realizar una unión y tomar el tiempo entre el inicio y el fin, desde que se opera la herramienta hasta que se concluye la junta.

- Para el segundo procedimiento se realizaron las uniones necesarias para tener un tramo de tubería de $60 \mathrm{~m}$, con una junta en cada extremo que podrá luego unirse a otro accesorio y se registró el tiempo total requerido.

En ambos procedimientos se ha utilizado un cronómetro Steren modelo CLK-150. Cabe indicar que las condiciones exteriores (temperatura ambiente, presión atmosférica) para los dos procedimientos fueron similares.

\subsection{Relación de costos}

Un aspecto fundamental que toma en cuenta un usuario de este tipo de sistemas es el costo de instalación, el cual depende directamente de:

- Precios de materiales (Tabla 1) y accesorios compatibles, más los fungibles, según los procedimientos de unión (Tabla 2). Estos precios han sido solicitados a tres proveedores locales y se ha escogido la cotización de menor valor.

- Mano de obra calificada que realiza las uniones y montajes (en procedimientos soldados, el personal debe calificarse ante un organismo certificador). Estos costos han sido obtenidos con la ayuda de empresas instaladoras con varios años de experiencia en sistemas de GLP.

- Mantenimiento preventivo que cada cinco años debe realizarse en una instalación [35]. Estos valores de mantenimiento han sido estimados en instalaciones de similar tamaño para cada uno de los materiales (Tabla 1) y formas instaladas de tubería (Tabla 3). 
La relación de costos expresa un valor tomado como unidad, y a partir de este según el criterio expresado, se comparan tantas veces contenga la unidad para los siguientes valores.

\section{Resultados y discusión}

\subsection{Propiedades del material}

Cada material responde a determinadas características, propiedades físicas y químicas, que hacen que sean más ventajosas que otras para una determinada aplicación.

\section{a) Resistencia a la tracción}

Con este valor se puede determinar qué carga puede soportar cada uno de ellos antes de rebasar su límite elástico y deformarse sin perder sus propiedades iniciales. La Figura 1 muestra la resistencia a la tracción de los diversos materiales aptos para conducción de GLP.

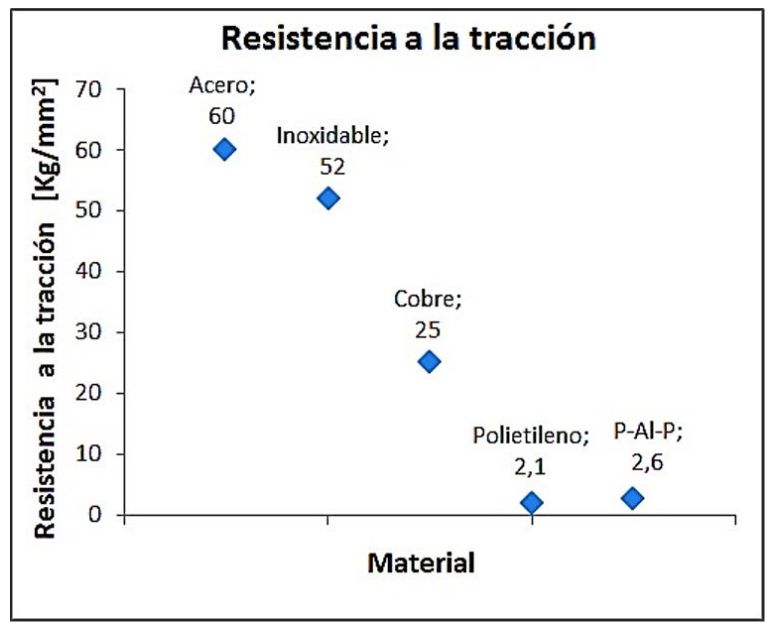

Figura 1. Comparación de la resistencia a la tracción.

El material con mayor resistencia a la tracción es el acero con $60 \mathrm{~kg} / \mathrm{mm}^{2}$, seguido por el acero inoxidable con $52 \mathrm{~kg} / \mathrm{mm}^{2}$, luego el cobre con $25 \mathrm{~kg} / \mathrm{mm}^{2}, \mathrm{P}-\mathrm{Al}-$ $\mathrm{P}$ con $2,6 \mathrm{~kg} / \mathrm{mm}^{2} \mathrm{y}$, finalmente, el polietileno con $2,1 \mathrm{~kg} / \mathrm{mm}^{2}$.

\section{b) Dureza}

Dureza es la propiedad de oposición de los materiales a ser rayados o penetrados, y como una tubería para conducción de GLP está expuesta a rayaduras y golpes por agentes externos, se muestra esta propiedad en la Tabla 4.

Con los resultados de a) y b), la tubería de acero es la más recomendada para instalarla en zonas de alto riesgo de golpes. Por tener la menor resistencia y por su degradación a la exposición a los rayos solares, es que la tubería de polietileno es recomendada para instalarla enterrada.
Tabla 4. Dureza de materiales

\begin{tabular}{ccc}
\hline Tubería & Dureza & Escala \\
\hline Acero al carbono & 99 & Rockwell B \\
Acero inoxidable & 92 & Rockwell B \\
Cobre & 37 & Rockwell B \\
Polietileno & 68 & Rockwell C \\
P-Al-P & $11^{*}$ & Rockwell B \\
\hline * Dureza del aluminio &
\end{tabular}

\section{c) Peso}

El peso por unidad de longitud de tubería para un diámetro de 1 " determina cuáles dan mejores prestaciones cuando se tienen que realizar instalaciones en altura, o para trayectos largos que sean manejables para cuadrillas de instaladores.

Con respecto al peso, la tubería de polietileno es la más liviana $(0,146 \mathrm{~kg} / \mathrm{m})$, lo cual la hace muy versátil al momento de instalar en tramos largos (Figura 2), y al venir en rollos, su instalación es muy sencilla. Algo similar sucede con la tubería P-Al-P, con un peso de $0,293 \mathrm{~kg} / \mathrm{m}$, es muy versátil para tramos largos, considerando que esta tubería puede instalarse embebida en paredes y pisos, por ductos y encamisadas.

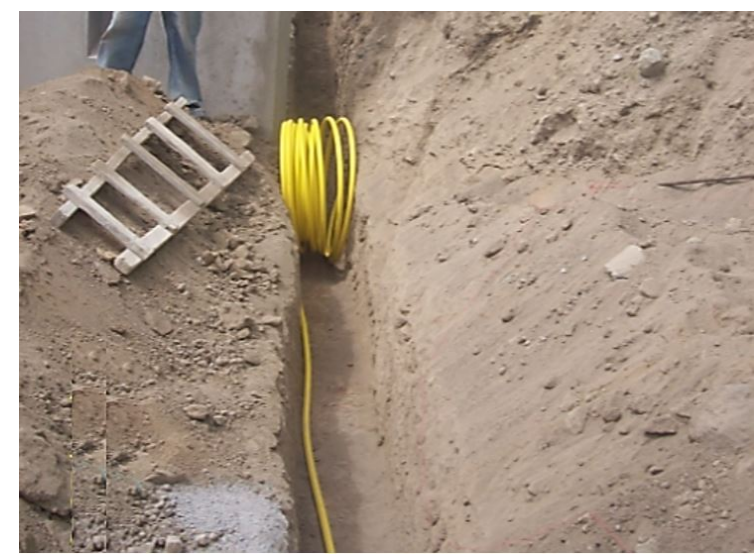

Figura 2. Tubería de polietileno lista a instalarse.

Sigue la tubería de acero inoxidable con $0,675 \mathrm{~kg} / \mathrm{m}$, luego la tubería de cobre con $0,997 \mathrm{~kg} / \mathrm{m}$ y, finalmente, la tubería de acero, cuyo peso es de $2,478 \mathrm{~kg} / \mathrm{m}$ lo cual hace que para tramos largos sea necesaria la instalación de accesorios intermedios de unión como bridas o universales. Igualmente esto hace poco manejable en diámetros grandes para realizar trabajos en taller, lo que hace que solo se pueda trabajar las uniones de estas tuberías in situ.

La Figura 3 muestra la comparación de valores de los pesos por metro de tuberías para conducción de GLP.

La Tabla 5 muestra los tiempos de unión de tuberías con accesorios, tomados para los procedimientos descritos en 2.2 (una sola unión de tubería con accesorio y unión de $60 \mathrm{~m}$ lineales continuos de tubería). 
Tabla 5. Tiempos promedio para realizar juntas o uniones en tuberías para GLP

\begin{tabular}{cccccc}
\hline Tiempos & Acero & $\begin{array}{c}\text { Acero } \\
\text { inoxidable }\end{array}$ & Cobre & Polietileno & P-Al-P \\
\hline $\begin{array}{c}\text { Tiempo de } \\
\text { unión para } \\
\text { una junta }\end{array}$ & $18 \mathrm{~min}$ & $15 \mathrm{~s}$ & $1 \mathrm{~min}$ & $1 \mathrm{~min}$ & $1 \mathrm{~min}$ \\
$\begin{array}{c}\text { Tiempo de } \\
\text { unión para } 60 \mathrm{~m} \\
\text { de tubería }\end{array}$ & $4,5 \mathrm{~h}$ & $3 \mathrm{~min}$ & $30 \mathrm{~min}$ & $2,5 \mathrm{~min}$ & $2,5 \mathrm{~min}$ \\
\hline
\end{tabular}

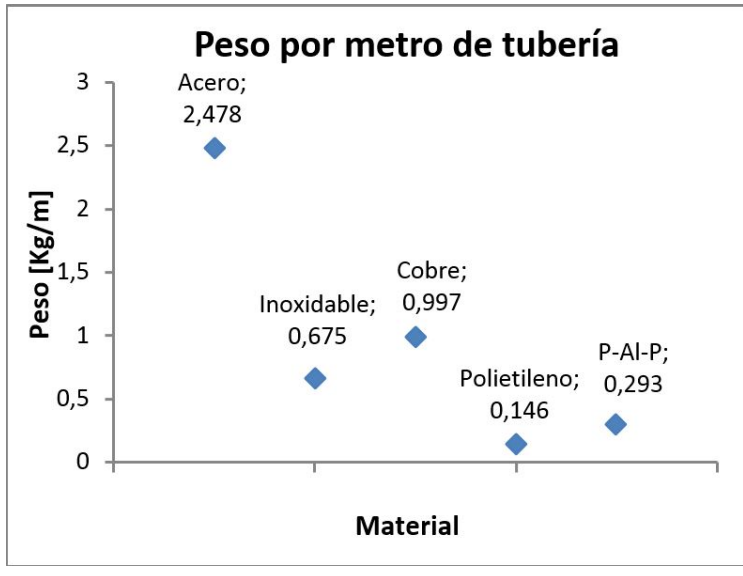

Figura 3. Comparación de pesos de tuberías para conducción de GLP

El primer procedimiento medido corresponde a una sola unión para tubería de 1" con una unión. La junta más rápida de realizar es la junta de pressing fit, su valor de $15 \mathrm{~s}$ corresponde al accionamiento de la herramienta, cierre de las tenazas, y posterior comprobación con las galgas pasa no pasa, es un proceso muy rápido y limpio. Los siguientes métodos tienen el mismo tiempo de unión: polietileno, P-Al-P y cobre con 1 min. El método incluye el calentamiento de la plancha para termofusión, que llegue a la temperatura exacta la tubería, y juntarla con el accesorio. Para el cobre, se toma en cuenta el tiempo de unión de la tubería con el accesorio por oxiacetileno, utilizando varilla de aleación de plata al $5 \%$, esta se funde, el líquido formado se vierte entre el tubo y el accesorio, consiguiendo la fusión. El método más largo es el SMAW en tubería de acero (18 min.), se considera los tiempos de 4 pases, desde la raíz, hasta el terminado final, incluida la limpieza de la escoria.

Para el segundo procedimiento los tiempos tomados son los necesarios para unir 60 metros de tubería. Los métodos más rápidos son polietileno y $\mathrm{P}-\mathrm{Al}-\mathrm{P}$ (2,5 min), ya que solamente se necesitan hacer dos juntas porque estas tuberías son continuas y vienen en rollos. Le sigue el método de unión para el acero inoxidable con pressing fit ( $3 \mathrm{~min})$, luego el método por capilaridad para la tubería de cobre con $30 \mathrm{~min}$ y, finalmente, el SMAW para la tubería de acero con 4,5 h.

\subsection{Relación de costos}

La Figura 4 muestra los costos asociados a las tuberías. Las medidas relacionadas son materiales, mantenimiento y mano de obra. Este análisis considera costos relativos, tomando como unidad a aquellos costos más bajos (en materiales y mano de obra), mientras que en el ítem de mantenimiento se toma como 1 al costo más bajo para tubería vista, considerando que las tuberías plásticas (polietileno y $\mathrm{P}-\mathrm{Al}-\mathrm{P}$ ) solo pueden instalarse ocultas y casi no requieren mantenimiento.

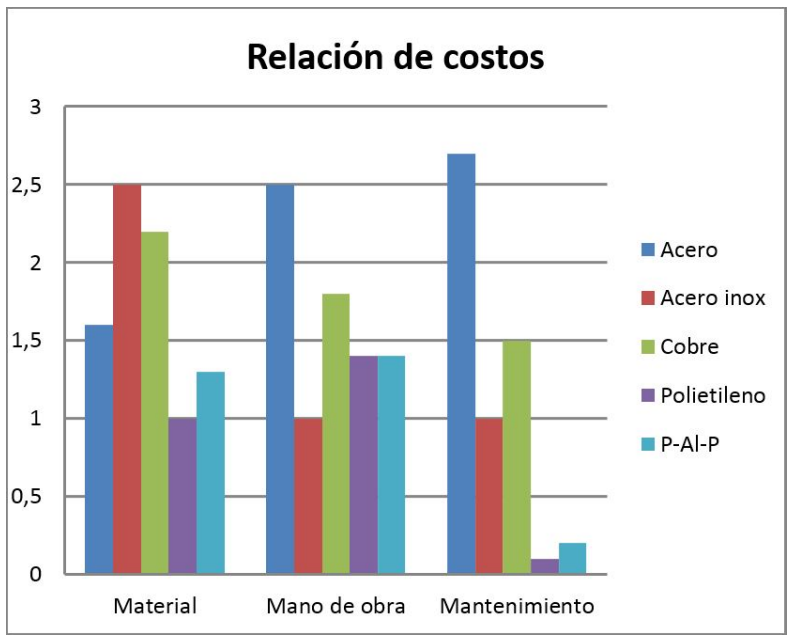

Figura 4. Relación costos de tuberías para servicios de GLP.

Los resultados de los costos presentados en la Figura 4 se pueden interpretar de la siguiente manera:

\section{a) Material}

El valor 1 lo tiene la tubería de polietileno; 1,3 veces más costosa es la tubería P-Al-P; 1,6 veces más costosa es la de acero; 2,2 la de cobre; y 2,5 más costosa es la de acero inoxidable.

\section{b) Mano de obra}

El menor rubro corresponde a la unión de tuberías de acero inoxidable mediante pressing fit, ya que este 
método de unión «solamente» implica accionar un dispositivo para cierre de unas mordazas, el instalador no requiere mayor habilidad ni capacitación, por tanto, su costo es el más bajo de todos.

Le siguen las tuberías de polietileno y P-Al-P con 1,4 ya que la termofusión, si bien no es un procedimiento muy complicado de ejecutar, necesita de mayor habilidad por parte del instalador que para el caso del acero inoxidable. Luego con un valor de 1,8 sigue la tubería de cobre, en cuyo método el instalador requiere mayor habilidad, debe ser calificado y certificado según lo establece la norma técnica [15].

Finalmente, el costo de mano de obra más elevado $(2,5)$ corresponde a la tubería de acero que requiere para su unión del método por arco eléctrico (SMAW), cuyo instalador es una persona con mucha habilidad, experiencia, calificado y certificado.

\section{c) Mantenimiento}

El valor unitario (para tuberías que pueden instalarse vistas) corresponde a la tubería de acero inoxidable, su mantenimiento es mínimo, ya que no se corroe y estéticamente es agradable a la vista. Con 1,5 le sigue la tubería de cobre, la cual en tramos vistos requiere mayor cuidado en protección.

Finalmente, está con 2,7 la tubería de acero al carbono, ya que en el mantenimiento de la misma se debe prever la repintura, incluso total, para tramos que han sido instalados en ambientes altamente corrosivos.

$\mathrm{Al}$ ir enterrada, la tubería de polietileno tiene el valor más bajo $(0,1)$, ya que no requiere mantenimiento; con un valor de 0,2 se asigna a la tubería de P-Al-P considerando mantenimiento mínimo de limpieza, en tramos instalados en ductos.

\subsection{Otros factores de selección}

Que un determinado material tenga mejores propiedades mecánicas (resistencia) sobre otro puede indirectamente ser factor de selección en tuberías de conducción de GLP, por ejemplo:

- Al tener mayor resistencia mecánica las tuberías metálicas (acero, acero inoxidable y cobre) resisten mejor a la mordedura de roedores que las tuberías plásticas, y esto es de suma importancia, sobre todo en instalaciones que están en zonas abiertas o en ductos, donde la probabilidad de presencia de estos animales es alta [31].

- Cuando una tubería va instalada vista, esta debe ser señalizada de acuerdo con la normativa respectiva y pintada con el color respectivo [36], amarillo ocre para conducción de GLP en fase vapor y blanca para conducción de GLP en fase líquida (Figura 5), con el fin de alertar a alguien ajeno a la instalación el peligro asociado al combustible.

- De igual manera, en tuberías ocultas es importante protegerlas y señalizarlas, así como a los elementos que se ubiquen como protección. De esta forma, y en caso de trabajos en sus alrededores, primero se encontrarán con las protecciones señalizadas antes que con las tuberías.

- Desde el punto de vista estético las tuberías de acero inoxidable tienen mejor calificación que las de acero al carbono.

- La resistencia al crecimiento de bacterias de las tuberías de acero inoxidable es superior al resto [31], lo cual las hace ideales en instalaciones donde se requiera pulcritud y asepsia.

- Hay factores externos que inciden en la selección de un material determinado en una instalación como disponibilidad de todos los materiales y accesorios en los proveedores al momento de requerirlos, disponibilidad del personal calificado para realizar las instalaciones, entre otros.

- Adicionalmente un costo indirecto de una instalación es el costo de los equipos y herramientas necesarios para realizar las juntas de tuberías con accesorios. Este costo, sin embargo, no ha sido tomado en cuenta para este análisis ya que la asignación de este valor a un proyecto determinado mucho dependerá de la proyección que tenga la empresa instaladora para trabajos a realizar en un determinado periodo de tiempo. Además, cada proyecto tiene su tiempo de ejecución distinto, lo que hace que la depreciación tanto de maquinarias como herramientas sea difícil determinar.

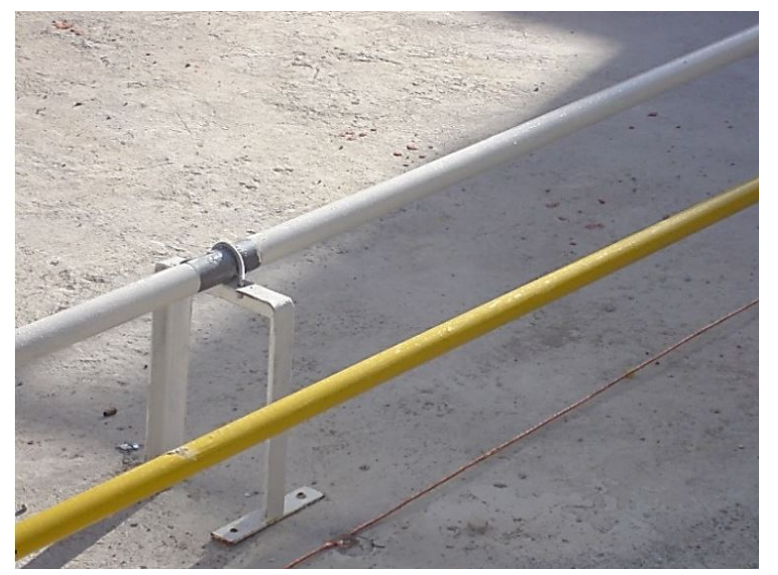

Figura 5. Señalización de tuberías de GLP (amarillo GLP vapor, blanco GLP líquido). 


\section{Conclusiones}

Cada proyecto tiene una realidad diferente por su ubicación, lugar por donde colocar la tubería, accesibilidad de materiales en el mercado, disponibilidad del personal idóneo para realizar el trabajo que hacen que la selección de la tubería adecuada sea específica para cada proyecto.

No se puede decir que un material es perfecto en una instalación, ya que son muchos los factores que hacen factible su selección, sin embargo, y dependiendo de las condiciones particulares de cada instalación, hay uno que tendrá varias ventajas sobre otros.

Es importante indicar que el usuario que requiere una instalación de GLP, y al tratarse de un combustible donde se debe tener en cuenta criterios de seguridad, el personal que debe realizar dicha instalación debe ser calificado y certificado ante la autoridad competente.

El instalador de un sistema de tuberías para GLP es quien debe garantizar la consistencia técnica utilizando materiales, procedimientos de unión, y formas correctas de instalar tuberías según las condiciones propias de cada proyecto, y es quien debe evaluar las mejores alternativas según el caso.

Juntando los criterios de resistencia mecánica y dureza nos permitirá valorar de mejor manera aplicaciones de tuberías cercanas a zonas de flujo vehicular, transporte de mercancías, que en un momento determinado pueden incidir directamente y causar daño en dichas tuberías.

Todas las comparaciones realizadas obedecen a una instalación de tubería y accesorios con personal calificado y bajo los criterios mínimos establecidos en la norma técnica. Una tubería de GLP debe quedar hermética y libre de fugas, por lo que realizar el procedimiento de unión correctamente va a reducir los riesgos asociados al transporte del combustible a través de las tuberías. Las tuberías de polietileno están recomendadas para instalarlas «enterradas» solamente, nunca puede instalarse vista.

\section{Recomendaciones}

Como un requerimiento imprescindible previo al suministro del combustible en una instalación nueva, sin importar el material empleado, el método de unión utilizado y la forma de instalación, se debe realizar una verificación de hermeticidad de la tubería bajo una presión de prueba mayor a la presión de trabajo.

Tuberías de P-Al-P pueden instalarse en ductos, embebidas o enterradas, es decir, ocultas, de ninguna manera puede instalarse vista ya que se ven afectadas por los rayos solares (cristalización) lo que hace que se fragilicen, puedan quebrase y provocar fugas.

Si una tubería enterrada es metálica, se deberá proporcionar un mecanismo efectivo para protegerla contra la corrosión a la cual puede estar expuesta.
Sin importar el material de tubería, si esta es instalada oculta, se la debe proteger por algún mecanismo cuya resistencia mecánica sea superior a la de dicha tubería, con el objetivo que si alguna persona involuntariamente realiza alguna actividad en las cercanías, primero se encuentre con este mecanismo que evite el daño en la tubería.

Si un usuario que necesita este tipo de instalación tiene varias propuestas comerciales, deberá buscar asesoría externa calificada que le ayude a escoger la mejor opción.

\section{Referencias}

[1] K. J. Morganti, T. M. Foong, M. J. Brear, G. da Silva, Y. Yang, and F. L. Dryer, "The research and motor octane numbers of liquefied petroleum gas (LPG)," Fuel, vol. 108, Supplement C, pp. 797-811, 2013. [Online]. Available: https://doi.org/10.1016/j.fuel.2013.01.072

[2] P. Boggavarapu, B. Ray, and R. Ravikrishna, "Thermal efficiency of lpg and png-fired burners: Experimental and numerical studies," Fuel, vol. 116, Supplement C, pp. 709-715, 2014. [Online]. Available: https://doi.org/10.1016/j.fuel.2013.08.054

[3] K. Troncoso and A. S. da Silva, "Lpg fuel subsidies in latin america and the use of solid fuels to cook," Energy Policy, vol. 107, Supplement C, pp. 188-196, 2017. [Online]. Available: https://doi.org/10.1016/j.enpol.2017.04.046

[4] B. Creamer and R. Becerra., "Cuantificación de los subsidios de derivados del petróleo a los hidrocarburos en el Ecuador," in Petróleo al día. Boletín Estadístico del Sector de Hidrocarburos, vol. 2, pp. 9-26, 2016. [Online]. Available: https://goo.gl/3oPCQi

[5] L. Raslavičius, A. Keršys, S. Mockus, N. Keršenè, and M. Starevičius, "Liquefied petroleum gas (lpg) as a medium-term option in the transition to sustainable fuels and transport," Renewable and Sustainable Energy Reviews, vol. 32, Supplement C, pp. 513-525, 2014. [Online]. Available: https://doi.org/10.1016/j.rser.2014.01.052

[6] H. Rijpkema, H. Roebers, and M. Mekes, "Tuberiías de plástico para gas en edificios y las consecuencias de los incendios," in Conferencia Internacional de Tuberias de Plastico, 2012. [Online]. Available: https://goo.gl/cHEBh2

[7] R. K. Andadari, P. Mulder, and P. Rietveld, "Energy poverty reduction by fuel switching. impact evaluation of the lpg conversion program 
in indonesia," Energy Policy, vol. 66, Supplement C, pp. 436-449, 2014. [Online]. Available: https://doi.org/10.1016/j.enpol.2013.11.021

[8] F. Chica, F. Espinoza, and N. Rivera., "Gas licuado de petróleo como combustible alternativo para motores diésel con la finalidad de reducir la contaminación del aire," INGENIUS, Revista de Ciencia y Tecnología, no. 4, pp. 73-81, 2010. [Online]. Available: http://dx.doi.org/10.17163/ings.n4.2010.08

[9] M. Johnsen and G. Nardini, "Manual de seguridad: Aspectos técnicos de la inflamabilidad de los gases hidrocarburos," Programa de las Naciones Unidas para el Medio Ambiente, 2005. [Online]. Available: https://goo.gl/VpkGxa

[10] D. Venegas, "Seguridad en la instalación de sistemas de gases industriales (glp)," in II Congreso Internacional de Energía, XXIV Asamblea COPIMERA. Santo Domingo, República Dominicana, 2016. [Online]. Available: https://goo.gl/EZVwHj

[11] J. Moncada., "Riesgo o peligro," NFPA Journal Latinoamericano, 2015. [Online]. Available: https://goo.gl/yypQMZ

[12] D. Venegas and O. Farias., "La bleve, un motivo para la seguridad en las instalaciones de GLP," in $13^{e r}$ Congreso Iberoamericano de Ingeniería Mecánica. Lisboa, Portugal, 2017. [Online]. Available: https://goo.gl/32aSg3

[13] D. Venegas, M. Arrocha, S. Celi, J. Rocha, C. Ayabaca, and E. Mena., "Manejo inseguro del gas licuado de petróleo en Panamá," $I+D$ Tecnológico, vol. 13, no. 2, pp. 22-30, 2017. [Online]. Available: https://goo.gl/TU8uB3

[14] NFPA. (2014) NFPA 58 código del gas licuado de petróleo edición 2014. [Online]. Available: https://goo.gl/yS9rpu

[15] INEN, NTE INEN 2260:2010 Instalaciones de gases combustibles para uso residencial, comercial e industrial. Requisitos, 2da Rev, Servicio Ecuatoriano de Normalización Std., 2010. [Online]. Available: https://goo.gl/LHmvsu

[16] M. Leporini, A. Terenzi, B. Marchetti, G. Giacchetta, F. Polonara, F. Corvaro, and R. C. Grifoni, "Modelling the pressurization induced by solar radiation on above ground installations of lpg pipeline systems," Journal of Physics: Conference Series, vol. 923, no. 1, pp. 1-9, 2017. [Online]. Available: https://goo.gl/4ghqFL
[17] D. Venegas and C. Ayabaca, Instalaciones de gas licuado de petróleo. Editorial Académica Española, 2017. [Online]. Available: https://goo.gl/N4NE1f

[18] Rego, "Catálogo L-102SV, equipo de GLP y amoniaco anhidro," Tech. Rep., 2011. [Online]. Available: https://goo.gl/o5LiuF

[19] J. Fuentes and J. Celis, "Instalaciones de gas natural," 2004. [Online]. Available: https://goo.gl/J3HVij

[20] ASTM, ASTM A53/A53M - 12, Especificación normalizada para tubos de acero negro e inmersos en caliente, galvanizados, soldados y sin costura, ASTM International, West Conshohocken, PA Std., 2012. [Online]. Available: https://goo.gl/czayAt

[21] ISO, ISO 65:1981: Carbon steel tubes suitable for screwing in accordance with ISO 7-1, International Organization for Standardization Std., 1981. [Online]. Available: https://goo.gl/DBGVBf

[22] ANSI/CSA, ANSI LC 1-2016/CSA 6.26-2016, Sistemas interiores de tuberías de gas combustible que utilizan tuberías de acero inoxidable corrugado, CSA Group, Std., 2016. [Online]. Available: https://goo.gl/zkZias

[23] ASTM, ASTM A240 / A240M-17, Standard Specification for Chromium and Chromium-Nickel Stainless Steel Plate, Sheet, and Strip for Pressure Vessels and for General Applications, ASTM International, West Conshohocken, PA Std., 2017. [Online]. Available: https://goo.gl/JH3WuY

[24] ISO, ISO 1640:1974: Wrought copper alloys Forgings - Mechanical properties, International Organization for Standardization Std., 1974. [Online]. Available: https://goo.gl/Jde7eN

[25] ASTM, ASTM B88-16, Standard Specification for Seamless Copper Water Tube, ASTM International, West Conshohocken, ASTM International, West Conshohocken, PA Std., 2016. [Online]. Available: https://goo.gl/AVRQR3

[26] ISO, ISO 4437:200\%: Buried polyethylene (PE) pipes for the supply of gaseous fuels - Metric series - Specifications, International Organization for Standardization Std., 2007. [Online]. Available: https://goo.gl/PAuTNi

[27] ASTM, Mathematical modelling of solute segregation in solidifying materials, ASTM International, West Conshohocken, PA Std., 2016. [Online]. Available: https://goo.gl/xmMkni 
[28] AS, Polyethylene/aluminium and cross-linked polyethylene/aluminium macro-composite pipe systems for pressure applications., Australian Standard Std., 1994. [Online]. Available: https://goo.gl/jj3chW

[29] ISO, ISO 17484-1:2006: Plastics piping systemsMultilayer pipe systems for indoor gas installations with a maximum operating pressure up to and including $500 \mathrm{kPa}$ (5bar).Part. 1: Specifications for systems., International Organization for Standardization Std., 2006. [Online]. Available: https://goo.gl/AZpzoh

[30] D. Venegas, J. Yanez, S. Celi, C. Ayabaca, L. Tipanluisa, D. Bastidas, and M. Arrocha, "Materiales recomendados por las normas internacionales para utilizar en una instalación de GLP," in XI Congreso Nacional de Ingeniería Mecánica, Elche - España, 2016. [Online]. Available: https://goo.gl/bLb54Y

[31] D. Venegas, M. Melendrez, and M. Arrocha, "Materiales para instalaciones de gas licuado de petróleo según National Fire Protection Association (NFPA)," in Congreso Internacional de Metalurgia y Materiales 16 SAM CONAMET, 2016. [Online]. Available: https://goo.gl/E4DnGs
[32] ASTM, ASTM E8M-00b, Standard Test Methods for Tension Testing of Metallic Materials [Metric], ASTM International, West Conshohocken, PA Std., 2001. [Online]. Available: https://goo.gl/rH1ADB

[33] — ASTM E18 - 03: Standard Test Methods for Rockwell Hardness and Rockwell Superficial Hardness of Metallic Materials., ASTM International, West Conshohocken, PA Std., 2003. [Online]. Available: https://goo.gl/Me5Yjm

[34] —, ASTM D785 - 08: Standard Test Method for Rockwell Hardness of Plastics and Electrical Insulating Materials., ASTM International, West Conshohocken, PA Std., 2015. [Online]. Available: https://goo.gl/fQYos4

[35] D. Venegas, J. YÁnez, S. Celi, C. Ayabaca, L. Tipanluisa, D. Bastidas, and M. Arrocha, "Mantenimiento necesario en instalaciones de GLP," in XXI Congreso Nacional de Ingeniería Mec ánica, Elche-España, 2016. [Online]. Available: https://goo.gl/KfujuH

[36] INEN, NTE INEN-ISO 9095 Tubos de acero - Marcado de caracteres continuos y código de colores para la identificación de materiales., Servicio Ecuatoriano de Normalización Std, 2014. [Online]. Available: https://goo.gl/ixg1rn 


\title{
INCENTIVO A LA GENERACIÓN DISTRIBUIDA EN EL ECUADOR
}

\section{INCENTIVE PERTAINING TO ENERGY THE GENERATION DISTRIBUTED IN ECUADOR}

\author{
Jorge Patricio Muñoz-Vizhñay ${ }^{1, *}$, Marco Vinicio Rojas-Moncayo ${ }^{1}$, \\ Carlos Raúl Barreto-Calle ${ }^{1}$
}

\section{Resumen}

La reducción de los costos de la infraestructura solar es una de las principales razones de su crecimiento mundial. En Ecuador se requiere realizar reajustes al marco jurídico que incentive la instalación de pequeños emprendimientos solares fotovoltaicos (de clientes del servicio eléctrico) conectados a las redes de distribución de baja tensión para consumo propio, y los excedentes sean inyectados a la red. Se plantean tres modelos de negocios para la microgeneración distribuida fotovoltaica, mismos que consideran dos sistemas de medición aplicables: el primero denominado netmetering donde se determina el valor neto de la energía (diferencia entre la inyectada a la red y la consumida), y el segundo conocido como «Feed-in Tariff»-FIT donde se determina la energía inyectada a la red a un precio especial como incentivo. El costo de la energía producida por un sistema fotovoltaico en el Ecuador es de USD/kWh 0,1342 con la tasa de descuento del $7 \%$, el CF (factor de capacidad) $=15 \%$, mientras que con la tasa de descuento del $10 \%, \mathrm{CF}=20 \%$ el costo de la energía alcanza a USD/kWh 0,1229, valores que no contemplan el banco de baterías ni de los terrenos, estos valores son cada vez más competitivos en relación con las fuentes renovables no convencionales.

Palabras clave: solar, fotovoltaico, medición, microgeneración, red, armónico.

\section{Abstract}

Reducing solar infrastructure costs is one of the main reasons for its global growth. In Ecuador adjustments to the legal framework have to be made to encourage the installation of small photovoltaic solar structures for electricity customers connected to low voltage distribution networks for their personal consumption, and any surplus energy be injected into the grid. Three business models pertaining to the distributed microgeneration of PV have been considered, for which we consider two applicable measurement systems: the first one is called "net metering" where the net value of the energy (the difference between the one injected into the network and the one consumed) is determined, and the second known as "Feed-in Tariff" - FIT where the energy injected into the grid is set at a special incentive price. The cost of energy produced by a photovoltaic system in Ecuador is US$\mathrm{D} / \mathrm{kWh} 0.1342$ with a discount rate $7 \%, \mathrm{CF}$ (capacity factor) at $15 \%$, while a discount rate $10 \%, \mathrm{CF}$ at $20 \%$ the cost reduces to USD / $\mathrm{kWh} \mathrm{0.1229.} \mathrm{These} \mathrm{values}$ however, do not take into account the bank of batteries or the land, these values are increasingly more competitive in relation to non-conventional renewable sources.

Keywords: solar, photovoltaic, measurement, microgeneration, net, harmonic.

\footnotetext{
1,*Facultad de Energía, las Industrias y los Recursos Naturales no Renovables, Universidad Nacional de Loja, Ecuador.

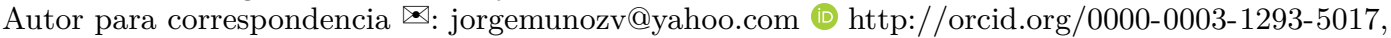

(D) http://orcid.org/0000-0002-1338-0095. (D) http://orcid.org/0000-0002-4886-2887.
} 


\section{Introducción}

El uso intensivo de fuentes de energía de origen fósil ha provocado impactos ambientales significativos en términos globales especialmente mediante las emisiones de $\mathrm{CO}_{2}$, uno de los principales gases responsables del calentamiento global del planeta o del denominado «efecto estufa o invernadero», causante de los cambios climáticos. Estudios realizados determinan que de un total de 1 trillón de toneladas de $\mathrm{CO}_{2}$ liberados en el planeta desde inicios de la industrialización, el $80 \%$ corresponde a emisiones de los últimos 50 años [1]. Por esta razón, cada vez más en el mundo las energías renovables no convencionales toman preponderancia, desarrollándose diferentes formas de aplicación como es el caso de la generación distribuida o embebida en los sistemas eléctricos de distribución. Generalmente, se define a la generación distribuida como a la generación de electricidad por plantas relativamente pequeñas (menor a $10 \mathrm{MW}$ ) en relación con las plantas centralizadas, con capacidad suficiente para permitir su interconexión en cualquier punto del sistema eléctrico considerando los siguientes aspectos: finalidad y localización; potencia nominal y nivel de tensión; características de la zona de entrega de energía.

Se estima que con la instalación de la generación distribuida se obtienen beneficios por la reducción de costos en pérdidas de transmisión y distribución en el orden del 5 al $10 \%$ de todos los $\mathrm{kWh}$ generados, existiendo, además, costos evitados en la expansión o repotenciación de los sistemas de transmisión y distribución, reducción de costos por mantenimiento de la infraestructura, aumento de confiabilidad a los consumidores próximos a la generación distribuida y atención más rápida al crecimiento de la demanda por tener menores tiempos de implementación en relación con la generación centralizada. Entre las principales desventajas de la generación distribuida se tiene la descoordinación de los equipos de protección, la desensibilización de las protecciones, dificultades en la reconexión, variaciones de tensión, sobretensiones, resonancia de sobretensión, armónicos [2].

\section{Análisis de la energía solar foto- voltaica a nivel mundial}

\subsection{Producción de energía solar fotovoltaica}

La oferta mundial de energía pasó de 6.642 millones de toneladas equivalentes de petróleo (TEP) en 1980, a 10939 millones de TEP en el 2005; a 12170 millones de TEP en el 2010; y, a 13105 millones de TEP en el 2015; con una tasa media anual de crecimiento del 1,8\%, en el último decenio (2005-2015) [3]. La matriz de energía mundial, en este periodo de 35 años, no presentó modificaciones estructurales significativas en lo que se refiere a la utilización de fuentes primarias de energía. De esta manera, es imprescindible considerar nuevas fuentes de energías primarias menos contaminantes como el caso del gas natural y las energías renovables. En este ámbito la energía solar fotovoltaica ha tenido en los últimos años el mayor crecimiento entre las energías renovables.

La capacidad instalada global de electricidad en paneles solares fotovoltaicos experimentó un crecimiento exponencial, alcanzando alrededor de 227 GWe para finales de 2015, produciendo cerca del $1 \%$ del total de electricidad o el 0,5\% del total de energía primaria en el mundo [3-15]. Este tipo de energía ha sido instalada principalmente en regiones con menos recursos solares (Europa y China), mientras que en regiones de altos recursos (África y Medio Oriente) sigue sin explotarse. Alemania durante la última década lidera la instalación de capacidad fotovoltaica, seguido de China, Japón, Italia y Estados Unidos. Las proyecciones realizadas para el 2050 de la energía solar, considera un alto nivel de penetración con lo cual la participación podrá estar entre el 18 y $31 \%$ de la generación total [4-17].

La reducción de los costos de la infraestructura solar es una de las principales razones del crecimiento mundial, los costos globales de los paneles fotovoltaicos cayeron en los Estados Unidos el 50 \% entre el 2006 y el 2011, siendo aún más acentuada esta caída del $60 \%$, entre el 2011 y el 2015 [5].

\subsection{Generación distribuida en redes de baja tensión tipo malla}

El comportamiento dinámico de la generación distribuida es diferente al de las máquinas (generadores) convencionales debido a que las constantes de tiempo de los elementos que las componen son pequeñas. En la actualidad se enfatiza el potencial de la generación distribuida de contribuir con potencia reactiva durante y después de una falla para mejorar la estabilidad de tensión de corto plazo. Sin embargo, la mayor preocupación radica en la desconexión frente a una perturbación. Consecuentemente es probable que las normativas impongan para las desconexiones inyección de potencia reactiva con el fin de salvaguardar la seguridad del sistema.

La concesionaria Consolidated Edison of New York Inc., una importante empresa de distribución americana que atiende áreas metropolitanas densamente pobladas, señala la dificultad de enviar energía a la red de baja tensión mallada con puntos de generación distribuida y sugiere usar adecuados sistemas de protección para eliminar posibles desconexiones; por tanto, recomienda, además, dimensionar la generación en relación con la demanda (manteniendo la generación distribuida siempre menor que la mínima demanda). Los sistemas de protección a usarse pueden ser relés de carga mínima o relés de potencia inversa, o la uti- 
lización de inversores controlados dinámicamente que modulan la generación de acuerdo con la carga [7].

La dificultad de enviar energía a la red se debe a la dificultad de coordinar los sistemas de protección de la generación distribuida con los de la red.

\subsection{Modelos de negocios de generación dis- tribuida (caso internacional)}

Para las empresas del sector eléctrico, los resultados no pueden ser solamente económicos, deben ser medidos también en términos de mejora de la calidad del servicio y los beneficios ambientales.

En el ámbito internacional y específicamente en el mercado solar norteamericano se aplican tres modelos de negocios. Actualmente, con la modernización del sector y la introducción de metas ambientales para la generación de energía han surgido formas híbridas de estos modelos.

Modelo 1. Las empresas eléctricas distribuidoras son propietarias de los activos de generación solar fotovoltaica y realizan la instalación, operación y mantenimiento de la infraestructura en locales de la propia empresa o de los clientes residenciales o comerciales pagando el alquiler de ocupación por el espacio o el tejado. La energía inyectada en la red pertenece a las empresas eléctricas.

Modelo 2. Las empresas eléctricas distribuidoras financian los sistemas de generación solar fotovoltaica a los clientes y otros actores considerando los altos costos de inversión inicial para la adquisición de los paneles y más equipos complementarios. Bajo este modelo la energía generada en exceso es inyectada a la red y los clientes pueden disfrutar de la compensación económica de la energía (neteo de la energía).

Modelo 3. Las empresas eléctricas distribuidoras contratan la energía solar fotovoltaica generada por terceros a través de los PPA (power purchase agreement), evitando relación con los microgeneradores (consumidores con generación). En este modelo las empresas distribuidoras realizan actividades tradicionales de contratación de energía para la reventa a los consumidores. Los contratos son establecidos con pocos generadores evitando relación con los microgeneradores o propietarios de tejados.

Para facilitar el uso de los recursos energéticos de la generación distribuida, es fundamental que los agentes del mercado mayorista y minorista tengan acceso transparente y no discriminatorio a las redes eléctricas y a la información de la medición. Esto puede considerarse como algo normal; sin embargo, las preocupaciones crecen cuando la empresa de distribución local, también es participante del mercado [8].

\subsection{Redes inteligentes para el control de la ge- neración distribuida}

La clave fundamental de un smart grid (redes inteligentes) es la integración de todos los elementos que forman parte de la red eléctrica. El concepto se refiere a la incorporación de tecnologías para sensar, monitorear, analizar la información de sus elementos y transmitirla en tiempo real para el mejor desempeño de las redes eléctricas, controlando los flujos de potencia entre los que se encuentra la generación distribuida, detectando fallas para provocar la reconexión automática sin afectar el desempeño. Esto permite que las áreas de coordinación de protecciones, control, instrumentación, medida, calidad y administración de energía, etc., sean concatenadas en un solo sistema de gestión con el objetivo primordial de lograr el uso eficiente y racional de la energía [9]. La implementación de sistemas smart grid en Ecuador está alineado con la ejecución de proyectos de microgeneración distribuida.

\subsection{Aspectos técnicos para la interconexión con la red eléctrica}

En las Figuras 1 y 2, se presentan formas de conexión entre el domicilio con generación fotovoltaica y la red eléctrica, estas tienen correspondencia con el modelo 2 descrito anteriormente. La Figura 3 corresponde al modelo 3 .

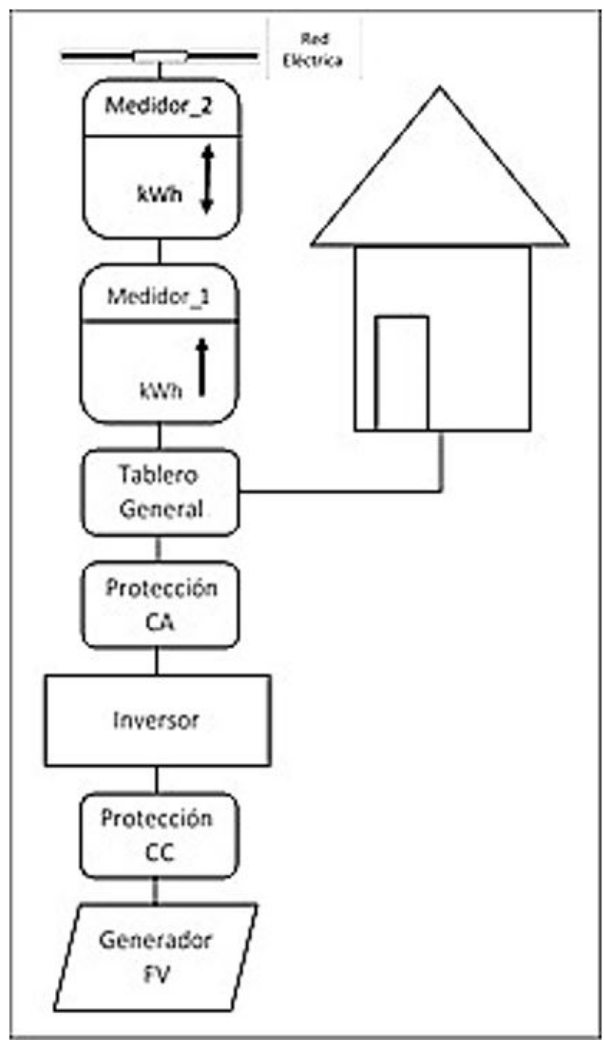

Figura 1. Punto de conexión recomendado cuando no hay incentivos a la generación fotovoltaica distribuida [9]. 
En la Figura 1, la energía fotovoltaica se entrega a la carga y el excedente se inyecta a la red eléctrica (sistema de distribución). El medidor 1 registra la energía inyectada a la red. La ventaja es que el medidor 2 gira en un sentido cuando el cliente consume energía y en sentido inverso cuando se inyecta a la red (el medidor determina el valor neto de energía), este sistema de medición es conocido como netmetering [9].

En sistemas radiales de distribución con generación distribuida, el flujo de potencia puede ser en sentido de subestación - carga, así como también en el sentido de carga - subestación, por tanto, la caída de tensión también puede ser en los dos sentidos. En función de la cantidad de generación fotovoltaica, para este último caso, podrían presentarse situaciones en los que se superen en algunos nodos los límites superiores de tensión.

En el caso de haber algún tipo de remuneración a los excedentes de la energía fotovoltaica, puede ponerse en marcha la conexión señalada en la Figura 2, previo a la regulación del precio de estos excedentes de energía (el medidor 1 registrará el excedente de energía inyectada a la red y el medidor 2 la energía consumida por el cliente pudiendo implementarse un solo medidor smart meter). Este sistema de excedentes de energía es conocido como «Feed-in Tariff»-FIT [9].

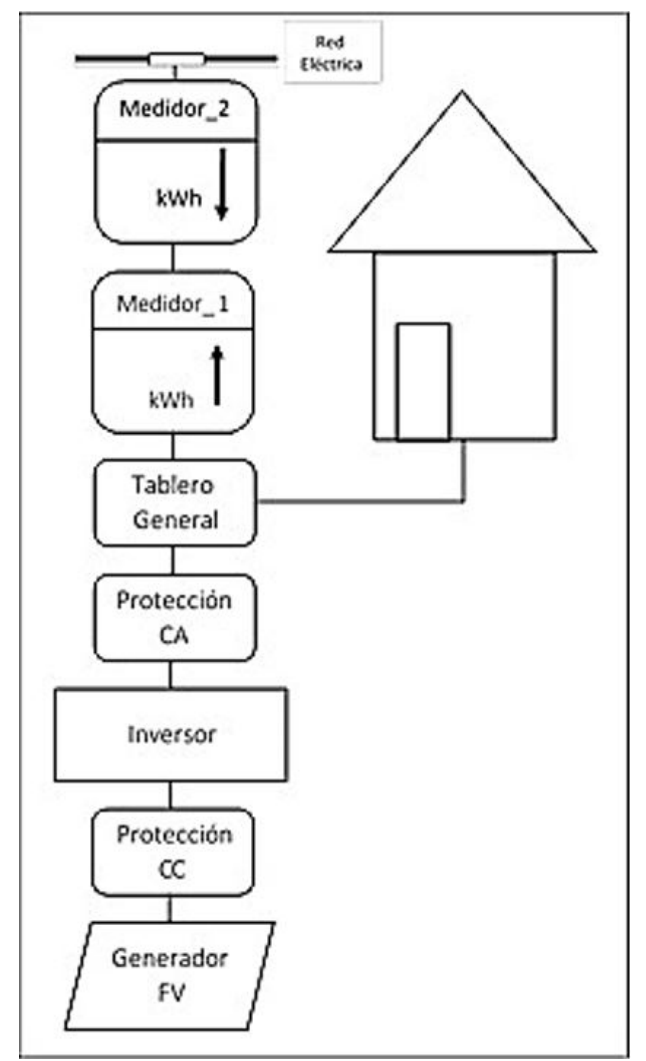

Figura 2. Punto de conexión recomendado cuando el excedente de la generación fotovoltaica distribuida tiene cierto incentivo [9].
En la Figura 3, correspondiente al modelo 3, al cliente se le factura conforme el consumo de energía (medidor 2) a la tarifa aplicable, y la generación fotovoltaica es vendida a la empresa eléctrica distribuidora a precio regulado para el efecto (el medidor 1 registrará la energía inyectada a la red).

Se considera que el modelo 3 , es el más factible de instalar en el Ecuador en los actuales momentos, debido a que los precios de la energía vendida por las empresas distribuidoras a los consumidores finales está afectado por un subsidio directo del Estado, lo cual impediría efectuar un neteo de los excesos de generación fotovoltaica en condiciones económicas adecuadas que garanticen la recuperación de la inversión.

Por lo tanto, resulta conveniente que el cliente sea facturado en forma independiente por la energía que consume de la red, y que la energía fotovoltaica que produzca sea facturada de manera independiente al precio adecuado que cubra los costos de producción fotovoltaica establecidos según la metodología indicada en el numeral 3.4.

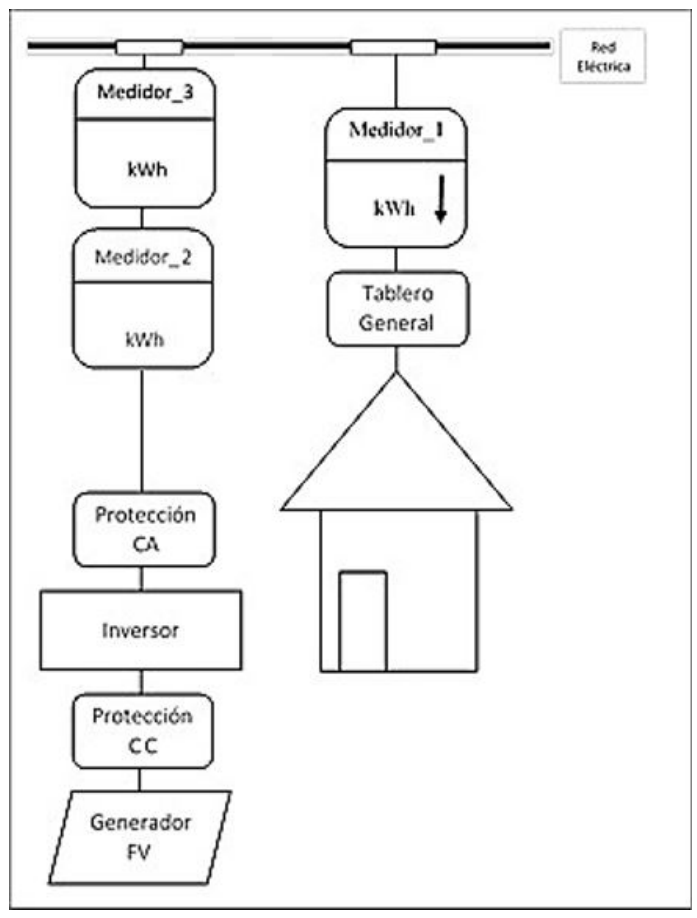

Figura 3. Punto de conexión recomendado cuando hay incentivos a la generación fotovoltaica distribuida [9].

\subsection{Incentivos a la generación solar foto- voltaica (experiencias internacionales)}

Algunos países están adoptando mecanismos de incentivo financiero para la energía solar fotovoltaica mediante la aplicación de sistemas tarifarios como la denominada tarifa feed-in que es el pago que realizan las empresas eléctricas distribuidoras por la energía 
generada e inyectada en las redes de distribución por parte de los clientes del servicio eléctrico [11].

El Gobierno federal norteamericano desde el 2006 prevé un descuento de los impuestos federales del $30 \%$ del costo de adquisición de los sistemas solares fotovoltaicos para clientes del tipo residencial y comercial. Además del incentivo federal, algunos estados ofrecen descuentos en otros impuestos [11]. Por ejemplo, la Florida propuso un precio premio a la energía a lo largo de 20 años con reducción gradual de la tarifa del $5 \%$ anual; Washington ofreció un incentivo a este tipo de energías para consumidores residenciales, comerciales e instituciones públicas, este incentivo consistió en un valor fijo (no ligado al $\mathrm{kWh}$ producido) por un periodo de 5 años [16]. No obstante, el sistema de compensación de energía (net metering) es también uno de los incentivos regulatorios usados para la energía solar fotovoltaica en los Estados Unidos. El modelo implanta el concepto que la energía activa producida con mini o microgeneración distribuida compensa el consumo de energía activa demandada de la red eléctrica por el cliente, es decir, que este paga a la empresa eléctrica distribuidora el valor neto que resulta entre la diferencia de la energía consumida y la energía generada [12].

El incremento per cápita de energía eléctrica en las áreas metropolitanas de las grandes ciudades contrasta con la dificultad cada vez mayor de construir en esas zonas líneas y redes de transmisión y distribución que en algunos casos deben ser soterradas, por esta razón la generación distribuida tendrá en el futuro un papel preponderante para regular la matriz energética de aquellas zonas.

En el caso de Brasil, mediante la expedición de un decreto en el 2004, por primera vez se crea la figura del generador distribuido, delineando el mercado a ser atendido. Ese mercado es constituido básicamente por las empresas eléctricas de distribución, mismas que pueden adquirir hasta el $10 \%$ de su demanda a los generadores distribuidos [8]. De esta manera, se abre un importante nicho de mercado para generación distribuida fotovoltaica especialmente cuando en clientes comerciales e industriales coincide la máxima generación con el pico de sus demandas.

Luego del desastre nuclear de Fukushima en Japón y la deshabilitación de reactores nucleares, este país estableció políticas de incentivos a la generación distribuida con energías renovables no convencionales especialmente la solar fotovoltaica, para lo cual a partir del 2012 puso en vigencia el modelo Feed-in Tariff (TIF). La normativa contempla precios para la energía entre 39,6 hasta 47,5 cUSD/kWh en función de la capacidad de generación [9-16].

Alemania es otro de los países que ha aplicado la generación distribuida aplicando el modelo Feed-in Tariff (TIF) para capacidades menores a $30 \mathrm{~kW}$, siendo obligación de las empresas distribuidoras otorgar el acceso y el pago de la energía inyectada a la red con precios de $24 \mathrm{cUSD} / \mathrm{kWh}$, además de un conjunto de subsidios estatales para la instalación [9-16].

En Inglaterra, al igual que en Japón y Alemania, los precios de la energía de la generación distribuida, mediante el modelo Feed-in Tariff (TIF), son mayores que los de la energía demandada para incentivar a los hogares a instalar paneles fotovoltaicos en sus casas. Los precios de la energía contemplan valores entre 19,8 a 24,3 cUSD/kWh para rangos de capacidades de hasta $50 \mathrm{~kW}[9-16]$.

En España fue implantado el sistema Feed-in Tariff (TIF) otorgando un precio premio calculado sobre la base de la tarifa de mercado, líneas de financiamiento, proveer condiciones especiales de inversión e incentivos fiscales [16].

La aplicación de precios preferenciales en estos países para generación distribuida ha sido adecuada, aumentando considerablemente la generación mediante energías renovables no convencionales, especialmente la solar fotovoltaica.

En Ecuador, la Ley Orgánica de Servicio Público de Energía Eléctrica no contempla la exoneración de aranceles, impuestos y más gravámenes que afecten la importación de materiales y equipos no producidos en el país, para la instalación de sistemas destinados a la utilización de energías renovables no convencionales como la energía solar.

\section{Análisis de la generación fotovoltaica en Ecuador}

\subsection{Potencial solar en el Ecuador}

El Ecuador a través del ex Consejo Nacional de Electricidad - CONELEC desarrolló el Atlas solar con fines de generación eléctrica. Los datos presentados en la Figura 4 representan la energía solar global promedio de los valores diarios de insolación total (directa y difusa), expresados en $\mathrm{Wh} / \mathrm{m}^{2} /$ día. En esta figura puede observarse las zonas con mayor insolación en el país y, por tanto, con mayor potencial para generación fotovoltaica como el caso de las provincias de Loja, Imbabura y Carchi [6].

El valor medio aproximado de la radiación solar global en el Ecuador es de $4.575 \mathrm{Wh} / \mathrm{m}^{2} /$ día.

$\mathrm{El}$ potencial solar estimado con fines de generación eléctrica en el país es de 312 GW equivalente a 456 TWh por año o 283 MBEP (millones de barriles equivalentes de petróleo) por año. Este valor equivale aproximadamente a quince (15) veces el potencial hidroeléctrico técnico y económicamente aprovechable del país.

A pesar de disponer el Ecuador de un alto potencial energético, el desarrollo de la energía solar fotovoltaica es aún incipiente, de manera particular en microgeneración distribuida; para septiembre de 2017, 


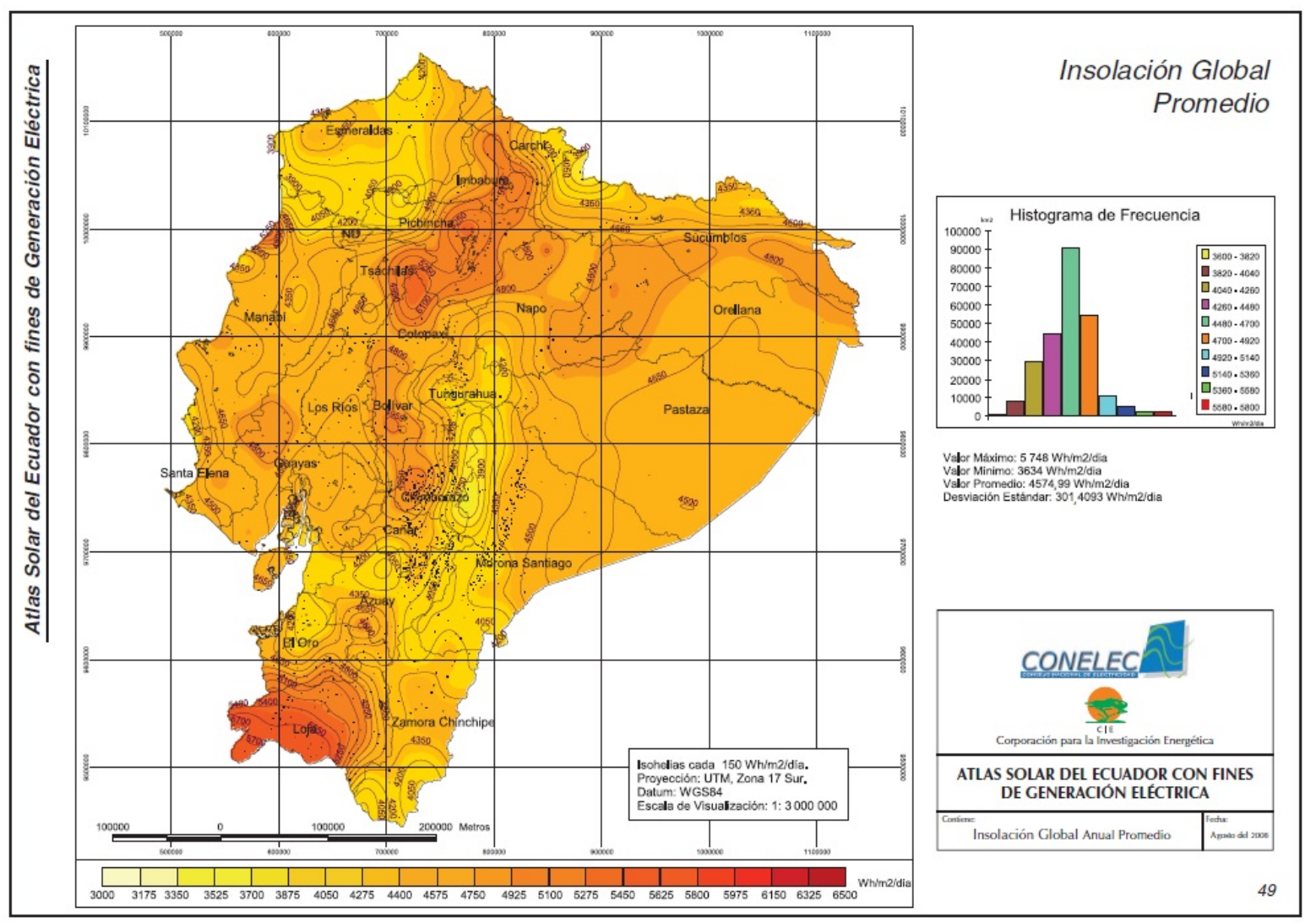

Figura 4. Mapa solar del Ecuador con fines de generación eléctrica [6].

el ARCONEL informa que la capacidad efectiva en este tipo de energía fue de 25,6 MW lo que representó el $0,34 \%$ de la capacidad total del país, habiendo producido 35,3 GWh/año equivalente al $0,15 \%$ de la producción total de energía. Además, en el Ecuador no se dispone de información relacionada con paneles fotovoltaicos que puedan considerarse como microgeneración distribuida. La información estadística antes indicada se refiere a centrales fotovoltaicas de capacidad entre 0,37 MW y $1 \mathrm{MW}$, siendo la gran mayoría de centrales cercanas a este último valor.

\subsection{Carga residencial de generación foto- voltaica}

Analizando la curva típica de carga residencial del Ecuador, en por unidad (pu) denominada por P en relación con la generación fotovoltaica en pu denominada FV, así como también el resultado de la diferencia de $\mathrm{P}-\mathrm{FV}$ (ver Figura 5), se determina que habrá flujo de energía en sentido de la red eléctrica al domicilio en horario aproximado a partir de las 16.30 hasta las 9.00 y el flujo será inverso (desde la generación FV a la red eléctrica) desde las 9.00 hasta las 16.30 aliviando el sistema de distribución. Este horario podría ser desplazado en el tiempo en función de la curva de carga P. En el momento (horario) de máxima demanda de carga residencial (P) (entre 19.00 y 20.00), la generación fotovoltaica $\mathrm{FV}$ no reduce el flujo de energía absorbido por el domicilio a la red eléctrica en razón de no estar contemplado un banco de baterías.

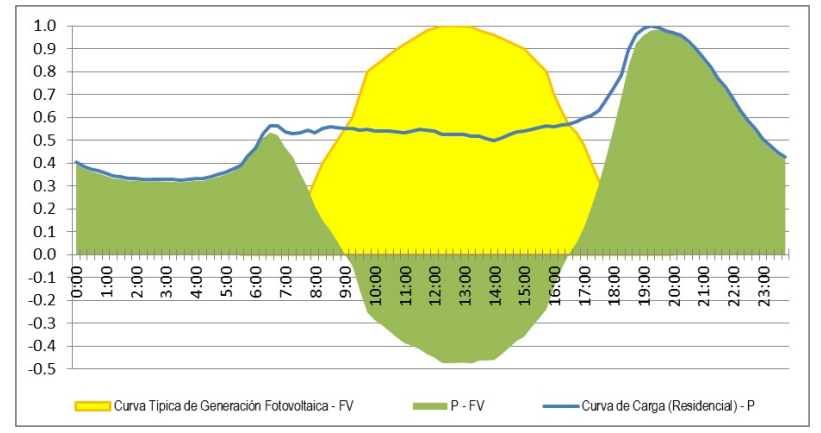

Figura 5. Curva de carga residencial resultante de la utilización de generación fotovoltaica [10].

El factor de potencia de la red eléctrica en el nodo de interconexión (red eléctrica-cliente) va disminuyendo progresivamente a medida que aumenta la generación fotovoltaica, en razón que la potencia activa va disminuyendo en tanto que la potencia reactiva será abastecida por la red (variación del triángulo de potencia). La norma IEC 61727 señala que el factor de potencia debe ser mayor a 0,9 inductivo cuando la carga del inversor fuere superior al $50 \%$. 
Las distorsiones armónicas de tensión introducidas en la red por la generación fotovoltaica son consecuencia de la caída de tensión proveniente de las corrientes armónicas producidas por el inversor que atraviesan las impedancias de la red. El total de armónicos deberá analizarse en función del número de paneles fotovoltaicos individuales conectados a la red. Según la norma IEC 61727 señala que la distorsión total de armónicos de corriente debe ser inferior al $5 \%$ en la salida del inversor.

Uno de los requerimientos específicos de la norma IEC 61727 relacionados a los niveles de tensión, indica que deberá estar en el rango comprendido entre el $85 \%$ y $110 \%$ de la tensión nominal de la red eléctrica. Al igual que la frecuencia deberá variar máximo $\pm 1 \mathrm{~Hz}$ de la frecuencia nominal de la red.

La inserción de paneles fotovoltaicos en las redes eléctricas en baja tensión tienen como consecuencia el aumento de la vida útil de los transformadores de distribución (MT/BT) por el alivio de carga, además que permite el ingreso de nuevos consumidores sin modificar su capacidad [10].

\subsection{Marco regulatorio ecuatoriano}

En el caso ecuatoriano, la Ley Orgánica de Servicio Público de Energía Eléctrica no especifica con claridad los aspectos, para que pequeños emprendimientos fotovoltaicos (personas naturales), conectados a las redes de distribución (baja tensión), puedan producir energía para el consumo propio y los excedentes para la comercialización a través de la red eléctrica. En este ámbito se determina la necesidad de contemplar en la ley, reglamentos, regulaciones, etc., la implementación de la generación distribuida con tecnologías renovables no convencionales especialmente la solar fotovoltaica para el sector residencial o doméstico por el alto potencial energético determinado por los niveles de insolación, otorgando diferentes tipos de incentivos. Las empresas eléctricas de distribución con base en la nueva normativa deberán facilitar la participación de la generación distribuida y llevar a cabo actividades de validación técnica ex ante, para asegurarse que no se produzcan restricciones en la red eléctrica y su verificación ex post [7].

Considerando como referencia a Japón y Alemania, mismos que establecieron tarifas más altas de la energía inyectada a la red en relación con el precio de facturación por el consumo del cliente, con el propósito de establecer incentivos en una real posibilidad de ahorro en el mediano y largo plazo en el pago de electricidad, este sistema de incentivo TIF presenta menor riesgo al inversionista y produce -al paso de los años- la reducción del incentivo (premio) conforme la disminución de costos de inversión de la tecnología energética, diferenciando el tamaño de la planta y la localización geográfica, de manera que permite dis- tribuir la tecnología de manera homogénea. Además, este instrumento regulatorio TIF ha sido ampliamente implantado en España y Dinamarca [13-16].

El Ecuador hasta hace poco aplicó la política de precios preferenciales para las fuentes renovables no convencionales (eólica, biomasa y biogás, geotérmica e hidroeléctrica) en la producción de electricidad que se podría decir corresponde a un sistema Feed-in Tariff, dicho incentivo se estableció mediante la Regulación Codificada N. ${ }^{\circ}$ CONELEC 001/13, misma que fue derogada en junio de 2016 [14]. En esta regulación no se contempló precio alguno para la energía solar fotovoltaica, notándose una fuerte deficiencia para la incorporación de este tipo de energía renovable.

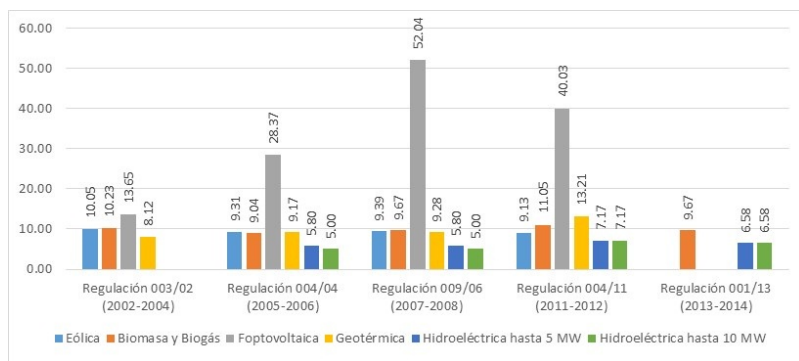

Figura 6. Precios de energía renovable en Ecuador.

En la Figura 6 puede observarse la evolución de los precios aprobados por el ex CONELEC en algo más de una década, donde el mayor precio para la energía solar fotovoltaica se presenta en el 2007 y 2008 (barras de color gris).

\subsection{Costos para implementación de genera- ción fotovoltaica en Ecuador}

El costo de generación fotovoltaica depende de los costos de inversión del equipamiento, los costos de operación y mantenimiento, la energía entregada por los paneles y el factor de capacidad.

Como ejemplo se ha tomado un proceso de contratación a través de la plataforma de compras públicas de $75 \mathrm{kWp}$. El costo medio de importación de los módulos alcanzó a USD/Wp 1,11 y los inversores a USD/Wp 0,32 . A esta cantidad se debe sumar los componentes nacionales de mano de obra, materiales de soporte de los módulos, cableado y el equipamiento de protección necesarios para la instalación, que en conjunto alcanza a USD/Wp 0,27 (no considera el costo del terreno ni baterías). De esta manera, el costo total de inversión para generación fotovoltaica es de USD/Wp 1,69. El costo de la energía puede ser calculado aplicando la siguiente expresión [8-11]:

$$
C=\left\{\left[\frac{r \times(1+r)^{N}}{(1+r)^{N}-1}\right]+O M\right\} \frac{I_{n v}}{8.76 \times C_{F}}
$$

Considerando, la tasa de descuento $\mathrm{r}=7 \%$ como costo de oportunidad; la vida útil del sistema $\mathrm{N}=20$ 
años; los costos anuales de operación y mantenimiento $\mathrm{OM}=1 \%$ del costo total de la inversión; la inversión inicial total $\mathrm{I}_{\mathrm{nv}}=1690 \mathrm{USD} / \mathrm{kWp} ; \mathrm{y}$, el factor de capacidad $\mathrm{CF}=15 \%$; de esta manera, el costo de la energía producida por el sistema fotovoltaico es de cUSD/kWh 13,42. Aplicando la misma expresión con el factor de capacidad $\mathrm{CF}=20 \%$ para otro lugar del Ecuador con altos niveles de insolación, el costo de la energía alcanza a cUSD/kWh 10,74. Estos valores son cada vez más competitivos con relación a la producción de energía hidroeléctrica y otras energías renovables no convencionales. Dependiendo del lugar geográfico en el que se instalen los paneles solares fotovoltaicos, el costo de producción de la energía podría variar entre los valores anotados. Se estima que para el año 2020, será posible que el costo de la energía solar fotovoltaica se reduzca a tal punto de convertirse en una fuente energética competitiva frente a las tecnologías convencionales. Concomitantemente deberán adoptarse políticas para evitar externalidades negativas frente a otros tipos de energías renovables no convencionales [16].

\subsection{Necesidad de crear un marco regulatorio en Ecuador para el desarrollo de la gene- ración fotovoltaica}

Es recomendable adecuar el marco jurídico del sector eléctrico ecuatoriano, mismo que permita incentivar la instalación de mini y microgeneración distribuida con el uso de energías renovables no convencionales especialmente la solar fotovoltaica considerando los adecuados niveles de insolación existentes en el Ecuador, permitiendo la inversión del sector privado en este tipo de iniciativas y creando los incentivos adecuados para que dicha inversión sea posible. Para el efecto, se deberá establecer, en primer lugar, la cuota de potencia que se pueda desarrollar en generación fotovoltaica para cada empresa distribuidora y cada tipo de cliente que no afecte la operación del sistema de distribución; y, los precios que se deban pagar por dicha producción, que si bien incorporen un incentivo a la inversión no causen un desequilibrio económico a las distribuidoras.

En las reformas del marco jurídico, se recomienda la aplicación del TIF para lo cual deberán establecerse precios de la energía inyectada a la red de distribución que podría estar en el orden de 20,0 cUSD/kWh y rangos de capacidades de hasta $4 \mathrm{~kW}$ para clientes residenciales, para un periodo de 20 años. El rango de capacidad para los clientes comerciales e industriales debe ser determinado con base en estudios que demuestren su factibilidad. Las empresas distribuidoras realizarán actividades tradicionales de contratación de energía para la reventa a los consumidores, lo que se podría denominarse como la aplicación del modelo 3 modificado señalado en el punto 2.3. De esta manera, los clientes que se acojan al programa tendrían bene- ficios económicos del orden 2:1 de la relación precio de la energía inyectada a la red versus el precio de facturación de la energía comprada a la distribuidora.

\section{Conclusiones y recomendaciones}

La generación de energía eléctrica distribuida a través de sistemas solares fotovoltaicos es una excelente alternativa para el gerenciamiento de la expansión de la oferta especialmente para modelos en los que se considera la generación distribuida.

En Ecuador no se dispone de un marco jurídico que incentive la participación de la mini y microgeneración distribuida con energías renovables no convencionales especialmente como el caso de la solar fotovoltaica.

Reformar el marco legal ecuatoriano que contemple los incentivos para la instalación de la generación distribuida por parte de los clientes residenciales, comerciales e industriales a través de sistemas de energías renovables especialmente la fotovoltaica. Entre los incentivos deberá considerarse la eliminación de aranceles en la importación de los equipos, precios preferenciales en la venta de energía a las empresas eléctricas distribuidoras y facilidad para la interconexión a las redes de distribución.

Se recomienda la aplicación del modelo Feed-in Tariff (TIF) para lo cual deberán establecerse precios de la energía inyectada a la red de distribución que podría estar en el orden de 20,0 cUSD/kWh, rangos de capacidades de hasta $4 \mathrm{~kW}$ para clientes residenciales, para un periodo de 20 años. El rango de capacidad para los clientes comerciales e industriales debe ser determinado basándose en estudios que demuestren su factibilidad.

El Ministerio de Electricidad y Energía Renovable se encuentra liderando el proceso de implantación en las empresas eléctricas de una serie de sistemas de automatización (SCADA, medición inteligente, DMSOMS, etc.) lo que para el futuro facilitará la instalación de microgeneración distribuida.

\section{Agradecimiento}

Se expresa un sincero agradecimiento a la Universidad Nacional de Loja y a la Facultad de Energía, las Industrias y los Recursos Naturales no Renovables por su apoyo incondicional a la realización de la investigación en el tema energético Diagnóstico y prospectiva de las energías que interactúan en la Zona 7 del Ecuador.

\section{Referencias}

[1] J. A. Aguilera, "Fuentes de energía y protocolo de Kioto en la evolución del sistema eléctrico español," Ph.D. dissertation, Universidad de Oviedo, 2012. [Online]. Available: https://goo.gl/s8A6g3 
[2] T. K. V. Hernández, "Uma proposta de integração da geração distribuida, por meio das usinas virtuais, ao sistema elétrico do estado de São Paulo," Master's thesis, Universidade de São Paulo, 2016. [Online]. Available: https://goo.gl/NXABQY

[3] IEA. (2017) Key world energy statistics. International Energy Agency. [Online]. Available: https://goo.gl/SHD6vM

[4] BP. (2017) Statistical review of world energy. [Online]. Available: https://goo.gl/PNqktm

[5] IRENA. (2017) Estadísticas de capacidad renovable 2017. International Renewable Energy Agency. [Online]. Available: https://goo.gl/Lp2F5b

[6] OLADE. (2008) Atlas solar del Ecuador con fines de generación eléctrica. Organización Latinoamericana de Energía. [Online]. Available: https://goo.gl/xyQQTt

[7] G. Barreto, "Metodología de aplicação de geração distribuída fotovoltaica em baixa tensão nos reticulados subterraneos das distribuidoras de energia elétrica," Ph.D. dissertation, Universidade de Sáo Paulo, 2014. [Online]. Available: https://goo.gl/W93aLG

[8] R. Benedito, "Caracterização da geração distribuída de eletricidade por meio de sistemas fotovoltaicos conectados á rede, no brasil, sob os aspectos técnico, econômico e regulatorio," Master's thesis, Universidade de São Paulo, 2009. [Online]. Available: https://goo.gl/q47eJV

[9] IRENA. (2017) Boosting global pv markets: The role of quality infrastructure. [Online]. Available: https://goo.gl/YyaRRa
[10] A. F. Pinto, "Avaliação dos impactos de sistemas fotovoltaicos conectados á rede de distribuição de baixa tensão," Ph.D. dissertation, Universidade de São Paulo, 2016. [Online]. Available: https://goo.gl/Qof7Xs

[11] M. P. Almeida, "Qualificação de sistemas fotovoltaicos conectados á rede," Master's thesis, Universidade de São Paulo, 2012. [Online]. Available: https://goo.gl/MQZ6Pw

[12] IRENA. (2017) Renewable energy benefits leveraging local capacity for solar pv. [Online]. Available: https://goo.gl/UhY1Yn

[13] — (2017) Adapting market design to high shares of variable renewable energy. [Online]. Available: https://goo.gl/iMmWei

[14] ARCONEL. (2017) Normativa y regulaciones. [Online]. Available: https://goo.gl/RFuerV

[15] World Energy Council. (2010) World energy. issues monitor 2017. [Online]. Available: https://goo.gl/B1eZvT

[16] S. M. K. Barbosa, "A competitividade das fontes energéticas em uma abordagemde learning curves: Uma proposição de regulação que incentive as tecnologias renováveis," Ph.D. dissertation, Universidade de São Paulo, 2016. [Online]. Available: https://goo.gl/u6i8AN

[17] World Energy Council. (2010) World energy perspectives. renewables integration 2016. [Online]. Available: https://goo.gl/xENr7B 


\title{
ON THE BEHAVIOUR OF SPHERICAL INCLUSIONS IN A CYLINDER UNDER TENSION LOADS

\author{
ESTUDIO DEL COMPORTAMIENTO DE \\ INCLUSIONES ESFÉRICAS EN UN CILINDRO \\ BAJO TRACCIÓN
}

\author{
Sebastián Montero ${ }^{1}$, Roger Bustamante ${ }^{1, *}$, Alejandro Ortiz-Bernardin ${ }^{1}$
}

\begin{abstract}
In the present paper the behaviour of a hyperelastic body is studied, considering the presence of one, two and more spherical inclusions, under the effect of an external tension load. The inclusions are modelled as nonlinear elastic bodies that undergo small strains. For the material constitutive relation, a relatively new type of model is used, wherein the strains (linearized strain) are assumed to be nonlinear functions of the stresses. In particular, a function is used that keeps the strains small, independently of the magnitude of the external loads. In order to simplify the problem, the hyperelastic medium and the inclusions are modelled as axial-symmetric bodies. The finite element method is used to obtain results for these boundary value problems. The objective of using these new models for elastic bodies in the case of the inclusions is to study the behaviour of such bodies in the case of concentration of stresses, which happens near the interface with the surrounding matrix. From the results presented in this paper, it is possible to observe that despite the relatively large magnitude for the stresses, the strains for the inclusions remain small, which would be closer to the actual behaviour of real inclusions made of brittle materials, which cannot show large strains.
\end{abstract}

Keywords: Nonlinear elasticity, Strain limiting behaviour, Finite element method, Constitutive equations, Elastic bodies, Isotropic bodies

\section{Resumen}

En el presente artículo se estudia el comportamiento de un sólido hiperelástico con una, dos y más inclusiones esféricas, bajo el efecto de una carga externa de tracción. Las inclusiones se modelan como sólidos elásticos con comportamiento no-lineal y que presentan pequeñas deformaciones, usando un nuevo modelo propuesto recientemente en la literatura, en donde las deformaciones (caso infinitesimal) se expresan como funciones no-lineales de las tensiones. En particular, se consideran expresiones para dichas funciones que aseguran que las deformaciones están limitadas en cuanto a su magnitud independientemente de la magnitud de las cargas externas. Como una forma de simplificar el problema, el medio hiperelástico y las inclusiones se modelan como sólidos axil-simétricos. El método de elementos finitos es usado para obtener resultados para estos problemas de valor de frontera. El objetivo del uso de los nuevos modelos para cuerpos elásticos para el caso de las inclusiones, es estudiar el comportamiento de dichos cuerpos en el caso de concentración de tensiones, lo cual ocurre cerca de la zona de interface con la matriz. De los resultados mostrados en este trabajo, es posible apreciar que a pesar de los valores relativamente altos para las tensiones, las deformaciones se mantienen pequeñas, lo cual sería mucho más cercano al comportamiento esperado en la realidad, cuando se trabaja con inclusiones hechas de un material frágil, el cual no puede mostrar grandes deformaciones.

Palabras clave: elasticidad no-lineal, límite para las deformaciones, método de elementos finitos, ecuaciones constitutivas, cuerpos elásticos, cuerpos isotrópicos.

\footnotetext{
1 ,* Department of Mechanical Engineering, University of Chile - Chile. Author for correspondence rogbusta@ing.uchile.cl. (D) https://orcid.org/0000-0002-2402-6139, (D) https://orcid.org/0000-0002-1072-1042 (D) https://orcid.org/0000-0001-9221-2470
}

Recibido: 10-10-2017, aprobado tras revisión: 19-12-2017

Forma sugerida de citación: Montero, S.; Bustamante, R.; Ortiz-Bernardin, A. (2018). «On the behaviour of spherical inclusions in a cylinder under tension loads». InGENIUS. N. ${ }^{\circ} 19$, (enero-junio). pp. 69-78. DOI: https: //doi.org/10.17163/ings.n19.2018.07. 


\section{Introduction}

In Refs. [1-3] Rajagopal and co-workers have proposed some new types of constitutive relations, which cannot be classified as either Green or Cauchy elastic equations. If $\mathbf{T}$ and $\mathbf{B}$ are used to denote the Cauchy stress tensor and the left Cauchy Green tensor, respectively, one of such relations is $\boldsymbol{f}(\mathbf{T}, \mathbf{B})=\mathbf{0}$, and two special cases that can be obtained from the above implicit relation are the classical nonlinear constitutive equation for a Cauchy elastic body [4] $\mathbf{T}=\boldsymbol{g}(\mathbf{B})$, and the subclass $\mathbf{B}=\boldsymbol{h}(\mathbf{T})$ (see, for example, Ref. [5]).

As a particular case, we assume that the gradient of the displacement field is small. From the above equation $\mathbf{B}=\boldsymbol{h}(\mathbf{T})$, we obtain $\varepsilon=\boldsymbol{g}(\mathbf{T})$, where $\varepsilon$ is the linearized strain tensor. This last constitutive equation is very important on its own, since it could be used to model the behaviour of some materials that can show a nonlinear behaviour, but where the strains are small, such as rock [6,7], concrete [8] and some metal alloys [9]. Another important use of $\boldsymbol{\varepsilon}=\boldsymbol{g}(\mathbf{T})$ is in the fracture mechanics analysis of brittle bodies [10], where for some particular expressions for $\boldsymbol{g}(\mathbf{T})$, it can be proved that for a crack in a brittle body, the magnitude of the strains are limited and do not go to infinite near the tip of a crack, contrary to what happens when the classical linearized elastic theory is used (see, for example, Ref. [11]). It is very important to study the behaviour of elastic bodies considering $\varepsilon=\boldsymbol{g}(\mathbf{T})$ for as many different boundary value problems as possible, in order to understand the capabilities and drawbacks of these new constitutive models, and that is the main objective of the present communication.

We are interested in studying the behaviour of a hyper-elastic (Green solid) cylindrical sample that can contain 1, 2 and 5 spherical inclusions, which are located in a row in the central axis of the cylinder, and which are equally separated from each other. The inclusions are assumed to behave as nonlinear elastic solids, using the new constitutive equation $\varepsilon=\boldsymbol{g}(\mathbf{T})$ mentioned above ${ }^{1}$. For simplicity the composite sample is modeled as an axial-symmetric body and a tension load is applied on the upper part of the cylinder. The finite element method is used to obtain results for the boundary value problem. We are particularly interested in studying the behaviour of the stresses and strains near the interface of the inclusions and the surrounding hyper-elastic body. It is assumed that the spherical inclusions are perfectly attached to the hyper-elastic matrix. The hypothesis of our work is that the new classes of constitutive equations $\varepsilon=\boldsymbol{g}(\mathbf{T})$ can be useful for the modelling of brittle bodies, in particular in the case we have large stresses, but where the strains must remain small. For a cylindrical sample with inclusions, such concentration of stresses appear near the interface of the matrix and the inclusion, and as it is shown in the present work, considering a particular expression for $\boldsymbol{g}(\mathbf{T})$, that we indeed obtain small strains for the spherical inclusions. For the modelling of such composite materials is very important to obtain results as precise as possible of the stresses near the interface, as the most common failure, which such composites show, corresponds to the debonding of the particles with the surrounding matrix.

This work is structured into the following sections. In Section 2 we present the basic equations for the models, in particular, the constitutive equations used for the spherical inclusions. In Section 3 we give details about the models to be analyzed. In Section 4, some numerical results for the different cases analyzed are presented. We end in Section 5 with some concluding remarks about the numerical results presented in this paper.

\section{Basic equations}

\subsection{Kinematics and equation of motion}

Let $X$ denotes a point of a body $\mathcal{B}$, the reference and current configurations are denoted as $\mathcal{B}_{r}$ and $\mathcal{B}_{t}$, respectively, and the position of point $X$ in such configurations is denoted as $\mathbf{X}$ and $\mathbf{x}$, respectively. It is assumed that there is a one-to-one mapping $\chi$ such that $\mathbf{x}=\boldsymbol{\chi}(\mathbf{X}, t)$, where $t$ is time. The deformation gradient $\mathbf{F}$, the left and the right Cauchy-Green tensors $\mathbf{B}, \mathbf{C}$, respectively, the Green Saint-Venant strain tensor $\mathbf{E}$, the displacement field $\mathbf{u}$ and the linearized strain tensor $\varepsilon$ are defined as:

$$
\begin{aligned}
\mathbf{F} & =\frac{\partial \boldsymbol{\chi}}{\partial \mathbf{X}}, \quad \mathbf{B}=\mathbf{F F}^{\mathrm{T}}, \quad \mathbf{C}=\mathbf{F}^{\mathrm{T}} \mathbf{F}, \\
\mathbf{E} & =\frac{1}{2}(\mathbf{C}-\mathbf{I}), \quad \mathbf{u}=\mathbf{x}-\mathbf{X}, \\
\boldsymbol{\varepsilon} & =\frac{1}{2}\left(\nabla \mathbf{u}+\nabla \mathbf{u}^{\mathrm{T}}\right) .
\end{aligned}
$$

Where $\nabla$ is the gradient operator with respect to the reference configuration. We assume $0<J<\infty$, where $J=\operatorname{det} \mathbf{F}$.

The equation of motion is

$$
\rho \ddot{\mathbf{x}}=\operatorname{div} \mathbf{T}+\rho \mathbf{b},
$$

where $\rho$ is the density of the body in the current configuration, $\mathbf{T}$ is the Cauchy stress tensor, $\mathbf{b}$ represents the body forces in the current configuration, $(")$ is the second derivative in time, and div is the divergence operator defined in the current configuration.

\footnotetext{
${ }^{1}$ See Ref. [12] for a recent work on the modelling of composites considering an extension of such new constitutive equations $\varepsilon=\boldsymbol{g}(\mathbf{T})$ for viscoelastic deformations.
} 
In our work we consider quasi-static deformations, therefore the left side of (4) is zero. More details about the above relations can be found, for example, in Ref. [13].

\subsection{Constitutive equations}

We consider a body composed of two materials, a matrix which is assumed to be hyper-elastic, filled with spherical inclusions which are assumed to behave as nonlinear elastic bodies undergoing small strains. For the hyper-elastic matrix cylinder, we assume there exists a function $W=W(\mathbf{F})$, called the energy function, such that (see, for example, Ref. [4])

$$
\mathbf{T}=J^{-1} \mathbf{F} \frac{\partial W}{\partial \mathbf{F}}
$$

where we use the convention $\left(\frac{\partial W}{\partial \mathbf{F}}\right)_{\alpha i}=\frac{\partial W}{\partial F_{i \alpha}}$. In this work, we use the neo-Hookean compressible model

$$
W=\frac{\mu}{2}\left(\bar{I}_{1}-3\right)+\frac{\kappa}{2}(J-1)^{2},
$$

where $\bar{I}_{1}=J^{-1 / 3} I_{1}, I_{1}=\operatorname{tr}(\mathbf{C})$, where tr is the trace of a second order tensor, and $\mu, \kappa$ are material constants.

For the inclusions, we assume that they are elastic bodies that develop nonlinear behaviour when strains are small. As indicated in the introduction section, recently some new types of constitutive relations for elastic bodies have been proposed in the literature [1-3], one of such relations is of the form

$$
\boldsymbol{f}(\mathbf{T}, \mathrm{B})=\mathbf{0},
$$

where the Cauchy elastic body $\mathbf{T}=\boldsymbol{g}(\mathbf{B})$ is a special subclass of the above relation, plus the new constitutive equation

$$
\mathrm{B}=\boldsymbol{h}(\mathbf{T}) .
$$

Assuming that $|\nabla \mathbf{u}| \sim O(\delta) \mid$ where $\delta \ll 1$, then $\mathbf{B} \approx 2 \varepsilon+\mathbf{I}$ (we also have $\mathbf{E} \approx \varepsilon$ ), and from (8) we obtain (see, for example, Refs. $[14,15]$ )

$$
\varepsilon=\boldsymbol{g}(\mathbf{T}),
$$

where in general $\boldsymbol{g}(\mathbf{T})$ is a nonlinear function of the stress tensor. We consider a special case of (9), where we assume there exists a scalar function $\Pi=\Pi(\mathbf{T})$ such that (see Ref. [16])

$$
\varepsilon=\boldsymbol{g}(\mathbf{T})=\frac{\partial \Pi}{\partial \mathbf{T}} .
$$

If $\Pi$ is assumed to be an isotropic function, we have that $\Pi(\mathbf{T})=\Pi\left(J_{1}, J_{2}, J_{3}\right)$, where $J_{i}, i=1,2,3$ are the following set of invariants of the stress tensor

$$
J_{1}=\operatorname{tr} \mathbf{T}, \quad J_{2}=\frac{1}{2} \operatorname{tr}\left(\mathbf{T}^{2}\right), \quad J_{3}=\frac{1}{3} \operatorname{tr}\left(\mathbf{T}^{3}\right) .
$$

And from (10), we obtain the representation

$$
\varepsilon=\Pi_{1} \mathbf{I}+\Pi_{2} \mathbf{T}+\Pi_{3} \mathbf{T}^{2},
$$

where $\Pi_{i}=\frac{\partial \Pi}{\partial J_{i}}, i=1,2,3$.

The following particular expression for $\Pi$ is considered:

$$
\Pi\left(J_{1}, J_{2}\right)=-\frac{\alpha}{\beta} \ln \left[\cosh \left(\beta J_{1}\right)\right]+\frac{\gamma}{\iota} \sqrt{1+2 \iota J_{2}},
$$

where $\alpha, \beta, \gamma$ and $\iota$ are constants.

Eq. (13) has been used in Ref. [17] to study problems, where independently of the magnitude of the stresses, the strains remains small. It is necessary to point out that this form for $\Pi$ and the numerical values of the constant that are shown in Chart 1, have not been obtained from experimental data. In Figures 1, 2 presented in Ref. [17], some plots for the behaviour of a cylinder under tension are shown, where it is possible to observe that the strains remain always small independently of the magnitude of the stresses. As indicated in the introduction section, such particular expressions could be important for fracture analysis of brittle bodies.

Finally, in Chart 1 the numerical values of the constants used in (6) and (13) are presented.

Chart 1. Values for the constants used in (6) and (13).

\begin{tabular}{cccccc}
\hline$\alpha$ & $\beta$ & $\gamma$ & $\iota$ & $\mu$ & $\kappa$ \\
& $1 / \mathrm{Pa}$ & $1 / \mathrm{Pa}$ & $1 / \mathrm{Pa}^{2}$ & $\mathrm{~Pa}$ & $\mathrm{~Pa}$ \\
\hline 0.01 & $9.277 \times 10^{-8}$ & $4.020 \times 10^{-9}$ & $10^{-14}$ & $80.194 \times 10^{6}$ & $150 \times 10^{6}$ \\
\hline
\end{tabular}

\subsection{Boundary value problems}

For the hyper-elastic cylinder, the boundary value problem is the classical formulation in nonlinear elasticity, where the function $\chi(\mathbf{X})$ is found by solving the equilibrium equation in the reference configuration (see, for example, Ref. [4] $)^{2}$ :

$$
\operatorname{Div} \mathbf{S}=\mathbf{0},
$$

where $\mathbf{S}=J^{-1} \mathbf{F T}$ is the nominal stress tensor. From (5), $\mathbf{S}=\frac{\partial W}{\partial \mathbf{F}}$, and Div is the divergence operator with respect to the reference configuration. Eq. (14) must be solved using the boundary conditions

$$
\mathbf{S}^{\mathrm{T}} \mathbf{N}=\hat{\mathbf{s}} \quad \mathbf{X} \in \partial \mathcal{B}_{r}^{s}, \quad \boldsymbol{\chi}=\hat{\mathbf{x}} \quad \mathbf{X} \in \partial \mathcal{B}_{r}^{x},
$$

where $\partial \mathcal{B}_{r}$ is the boundary of the hyper-elastic body in the reference configuration, $\partial \mathcal{B}_{r}^{s} \cup \partial \mathcal{B}_{r}^{x}=\partial \mathcal{B}_{r}$, $\partial \mathcal{B}_{r}^{s} \cap \partial \mathcal{B}_{r}^{x}=\varnothing, \mathbf{N}$ is the outward normal vector to the surface of the body in the reference configuration, $\hat{\mathbf{s}}$ is the external traction (described in the reference

\footnotetext{
${ }^{2}$ In this paper we do not consider the effect of body forces.
} 
configuration), and $\hat{\mathbf{x}}$ is a known deformation field on some part of the surface of the hyper-elastic body.

For the inclusions we consider small strains and displacements. And following what has been presented in Refs. [16,17], for the boundary value problem corresponds to find $\mathbf{T}$ and $\mathbf{u}$ by solving (see (3), (4) and $(10))$

$$
\operatorname{div} \mathbf{T}=\mathbf{0}, \quad \frac{1}{2}\left(\nabla \mathbf{u}+\nabla \mathbf{u}^{\mathrm{T}}\right)=\frac{\partial \Pi}{\partial \mathbf{T}}
$$

simultaneously. In the above system we have 9 equations for a fully 3D problem, and 9 unknowns that correspond to the components of the stress tensor and the displacement field. Regarding the boundary conditions we have in general

$$
\operatorname{Tn}=\hat{\mathbf{t}} \quad \mathbf{x} \in \partial \mathcal{B}_{t}^{t}, \quad \mathbf{u}=\hat{\mathbf{u}} \quad \mathbf{x} \in \partial \mathcal{B}_{t}^{u},
$$

where $\partial \mathcal{B}_{t}$ is the surface of the inclusion and $\mathbf{x} \in$ $\partial \mathcal{B}_{t}^{t} \cup \partial \mathcal{B}_{t}^{u}=\partial \mathcal{B}_{t}, \partial \mathcal{B}_{t}^{t} \cap \partial \mathcal{B}_{t}^{u}=\varnothing, \mathbf{n}$ is the normal vector to the surface of the inclusion, $\hat{\mathbf{t}}$ is the external load and $\hat{\mathbf{u}}$ is a known displacement field on a part of the surface of the inclusion. Since for the inclusion we assumed that $|\nabla \mathbf{u}| \sim O(\delta) \mid$ where $\delta \ll 1$, then there is no need for distinguishing between the reference and the current configuration for that body.

\section{Axial-symmetric models}

For simplicity, the hyper-elastic matrix is considered to be a cylinder of radius $R$ and length $L$ (see, for example, Figure 1).

For a cylinder with one inclusion, it is assumed that the inclusion is located in the center of the cylinder, and that the radius of that spherical body is $r_{i}$ (see Figure 1). It is assumed that there is axial symmetry, therefore, we study a plane problem using the coordinates $r$ and $z$ (radial and axial axis, respectively).

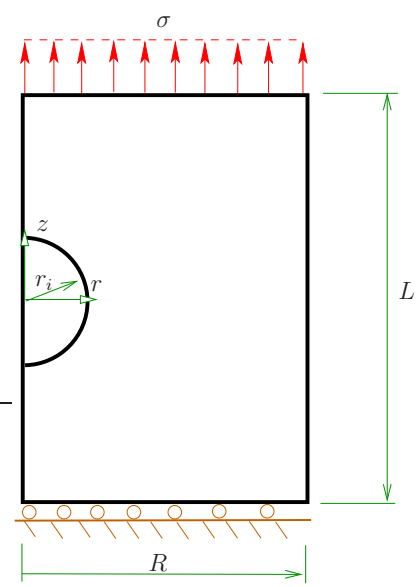

Figure 1. Hyper-elastic cylinder with one inclusion.

The center of the sphere is located at $z=L / 2$. On the surface $z=L$ we apply a uniform axial load $\sigma$. On the surface $z=0$ we assume that the cylinder cannot move in the axial direction, but it is free to expand in the radial direction, i.e., $u_{z}(r, 0)=0$. On the surface $r=R$ we assume that the cylinder is free. Finally, the spherical inclusion is assumed to be perfectly bonded to the the surrounding hyper-elastic cylinder, i.e., the displacement field is continuous across the surface of the inclusion.

In Figure 2, an hyper-elastic cylinder with two inclusions is depicted.

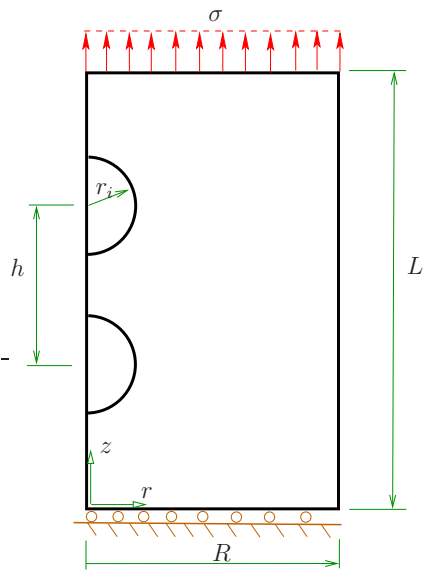

Figure 2. Hyper-elastic cylinder with two inclusions.

These two inclusions are of the same radius, are separated by a distance $h$ between centers (the central point between them is located in the middle of the cylinder in the axial line defining it.) The two inclusions are assumed to behave as nonlinear elastic bodies using (10). The rest of the boundary conditions for the problem are the same as the problem presented in Figure 1.

In Figure 3, the case of an hyper-elastic cylinder with five inclusions in a row is presented.

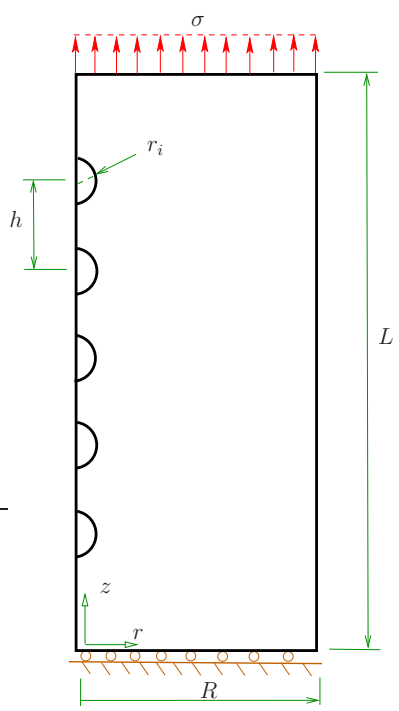

Figure 3. Hyper-elastic cylinder with five inclusions. 
The inclusions are separated to each other by the same distance $h$. As in the previous case, they are modelled using (10), and the center point for all the inclusions is located in the center of the cylinder.

For the different models mentioned previously, it was assumed that $r_{i}=1 \mathrm{~mm}$. Regarding $R, L$ and $h$, different cases were considered as indicated in Tables $2-4$.

Chart 2. Cases studied for the cylinder with one inclusion.

\begin{tabular}{|l|cccc|}
\hline$R$ & $2 r_{i}$ & $3 r_{i}$ & $4 r_{i}$ & $5 r_{i}$ \\
\hline$L$ & $6 r_{i}$ & $8 r_{i}$ & $10 r_{i}$ & \\
\hline
\end{tabular}

For the case of a cylinder with two inclusions, we assume $R=5 r_{i}$ and $L=10 r_{i}$ (the parameter $h$ is presented in Chart 3.)

Chart 3. Cases studied for the cylinder with two inclusions.

\begin{tabular}{|l|llllllll|}
\hline$h$ & $2.1 r_{i}$ & $2.2 r_{i}$ & $2.3 r_{i}$ & $2.4 r_{i}$ & $2.5 r_{i}$ & $3 r_{i}$ & $4 r_{i}$ & $5 r_{i}$ \\
\hline
\end{tabular}

Finally, for the case of a cylinder with five inclusions, we assume $R=5 r_{i}$ and $L=4\left(h+r_{i}\right)$, and for $h$ we have the cases presented in Chart 4 .

Chart 4. Cases studied for the cylinder with five inclusions.

$$
\begin{array}{|l|llllllllll|}
\hline h & 2.1 r_{i} & 2.2 r_{i} & 2.3 r_{i} & 2.4 r_{i} & 2.5 r_{i} & 3 r_{i} & 4 r_{i} & 5 r_{i} & 6 r_{i} & 7 r_{i} \\
\hline
\end{array}
$$

The boundary value problems were solved using the finite element method with an in-house finite element code (details of the method in which the code is based can be found, for example, in Ref. [16].)

\section{Numerical results}

\subsection{Results for one inclusion}

In this section we show some results for a cylinder with one spherical inclusion located on its center (see Figure 1), for the cases indicated in Chart 2.

In Figure 4, results are presented for the axial and radial components of the normalized stress and the components of the strain, for different values for $R$, for the case $L=10 r_{i}$.
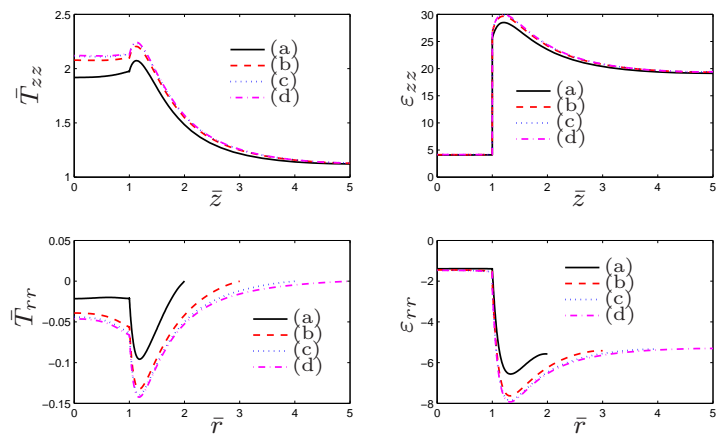

Figure 4. Results for the normalized components of the stress $\bar{T}_{z z}$ and strain $\varepsilon_{z z}$, for the line $r=0,0 \leq \bar{z} \leq \bar{L} / 2$, and the component $\bar{T}_{r r}$ of the stress and $\varepsilon_{r r}$ of the strain, for the line $z=0,0 \leq \bar{r} \leq \bar{R}$. This is for the case $L=10 r_{i}$, where: (a) $R=2 r_{i}$ (b) $R=3 r_{i}$ (c) $R=4 r_{i}$ (d) $R=5 r_{i}$.

The normalized components of the stress that appear in Figure 4 are defined as

$$
\bar{T}_{z z}=\frac{T_{z z}}{\sigma}, \quad \bar{T}_{r r}=\frac{T_{r r}}{\sigma},
$$

where $\sigma$ is the uniform load applied on the upper surface of the cylinder (see Figure 1). The strains are in $\%$.

The results for the axial components of the normalized stress and strain $\bar{T}_{z z}$ and $\varepsilon_{z z}$ presented in Figure 4, respectively, are shown for the line $r=0,0 \leq \bar{z} \leq \bar{L} / 2$, where

$$
\bar{z}=\frac{z}{r_{i}}, \quad \bar{L}=\frac{L}{r_{i}} .
$$

The results for the radial components of the normalized stress and strain $\bar{T}_{r r}$ and $\varepsilon_{r r}$ presented in Figure 4, respectively, are shown for the line $z=0$, $0 \leq \bar{r} \leq \bar{R}$, where

$$
\bar{r}=\frac{r}{r_{i}}, \quad \bar{R}=\frac{R}{r_{i}} .
$$

In Figure 5 we show similar results as in Figure 4, for $R=5 r_{i}$ and different cases for $L$.
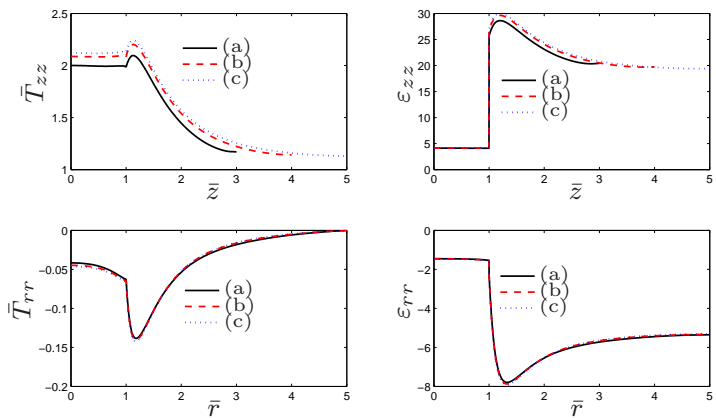

Figure 5. Results for the normalized components of the stress $\bar{T}_{z z}$ and strain $\varepsilon_{z z}$, for the line $r=0,0 \leq \bar{z} \leq \bar{L}$, and the component $\bar{T}_{r r}$ of the stress and $\varepsilon_{r r}$ of the strain, for the line $z=0,0 \leq \bar{r} \leq \bar{R}$. This is for the case $R=5 r_{i}$, where (a) $L=6 r_{i}$ (b) $L=8 r_{i}$ (c) $L=10 r_{i}$. 
The results for $\bar{T}_{z z}$ and $\varepsilon_{z z}$ are shown for the line $r=0,0 \leq \bar{z} \leq \bar{L}$, while for $\bar{T}_{r r}$ and $\varepsilon_{r r}$, the results are shown for the line $z=0,0 \leq \bar{r} \leq \bar{R}$.

In both Figures 4 and 5 the inclusion is located in the region $\bar{r} \leq 1, \bar{z} \leq 1$, and due to the symmetry of the problem, only the upper half part of the inclusion and the cylinder is considered (see Figure 1).

In Figures 6-9 we show results for the radial and axial components of the strain and the stress, for the case $R=5 r_{i}, L=10 r_{i}$. The stresses are presented in $\mathrm{Pa}$.

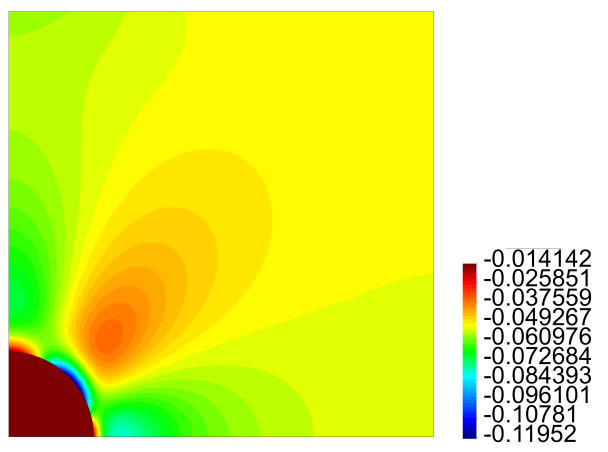

Figure 6. Contour plot for $\varepsilon_{r r}$ for the problem of one inclusion.

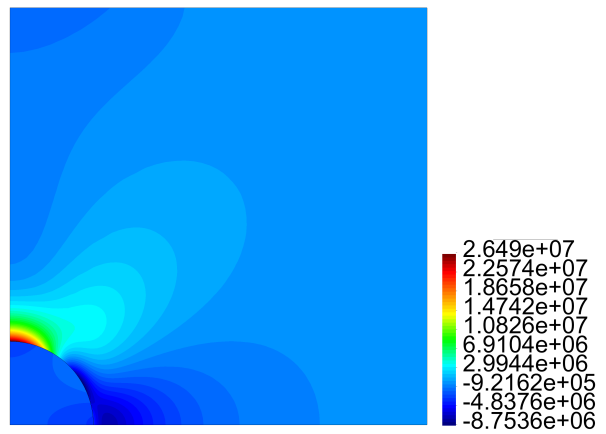

Figure 7. Contour plot for $T_{r r}$ in $\mathrm{Pa}$ for the problem of one inclusion.

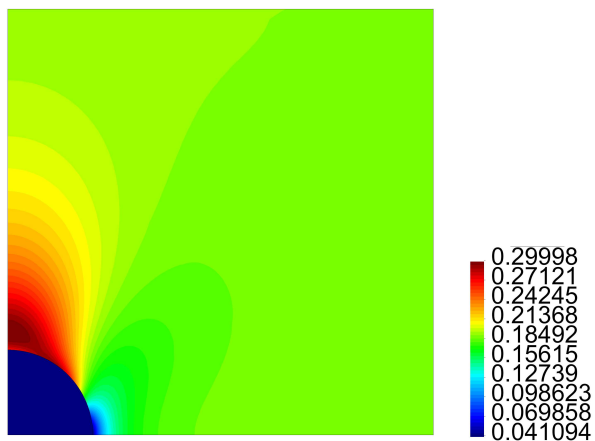

Figure 8. Contour plot for $\varepsilon_{z z}$ for the problem of one inclusion.

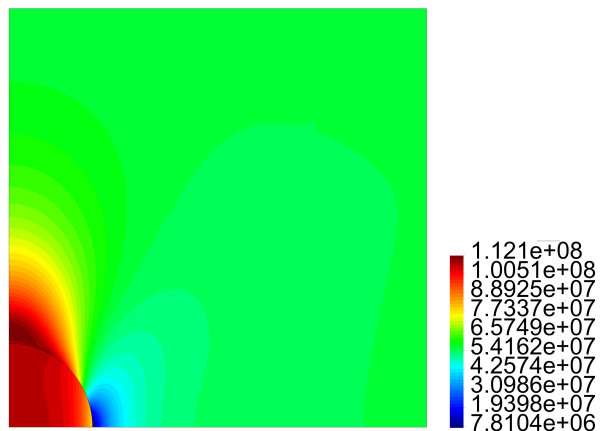

Figure 9. Contour plot for $T_{z z}$ in $\mathrm{Pa}$ for the problem of one inclusion.

\subsection{Results for two inclusions}

Figure 10 depicts results for the axial and radial components of the stress (normalized stresses, see (18)) and the strain, for the line $r=0,0 \leq \bar{z} \leq \bar{L}$, for different values for $h$ as presented in Chart 3 for a hyperelastic cylinder with two inclusions (see Figure $2)$.
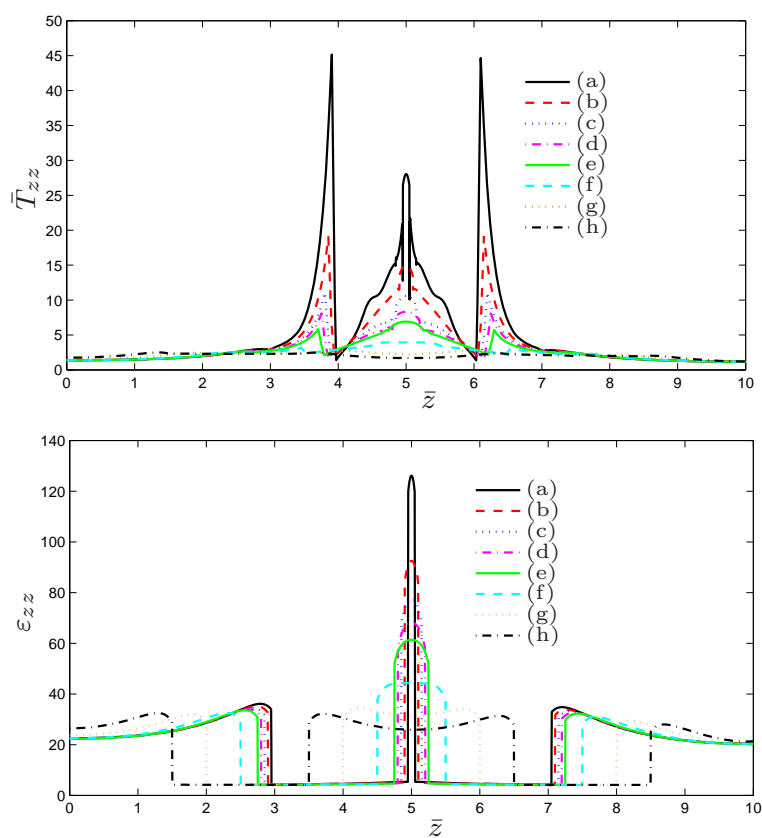

Figure 10. Results for the normalized components of the stress $\bar{T}_{z z}$ and strain $\varepsilon_{z z}$, for the line $r=0,0 \leq \bar{z} \leq \bar{L}$, where (a) $h=2.1 r_{i}$ (b) $h=2.2 r_{i}$ (c) $h=2.3 r_{i}$ (d) $h=2.4 r_{i}$ (e) $h=2.5 r_{i}$ (f) $h=3 r_{i}$ (g) $h=4 r_{i}$ (h) $h=5 r_{i}$.

In Figures 11-14 we present results for the radial and axial components of the strain and the stress, for the case $h=2.5 r_{i}$ (the stresses are in Pa.) 


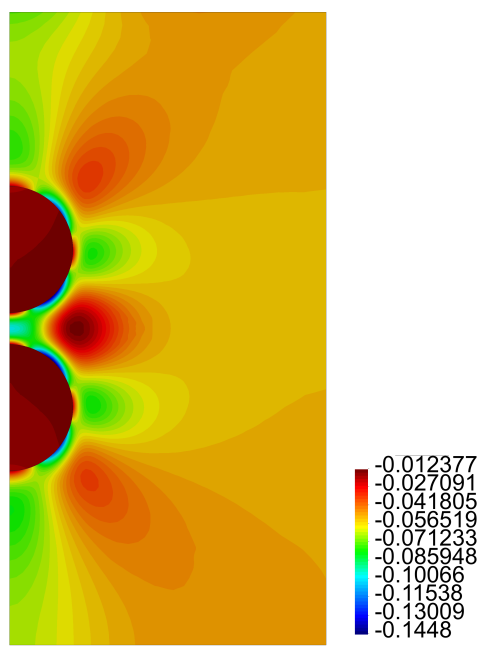

Figure 11. Contour plot for $\varepsilon_{r r}$ for the problem of two inclusions.

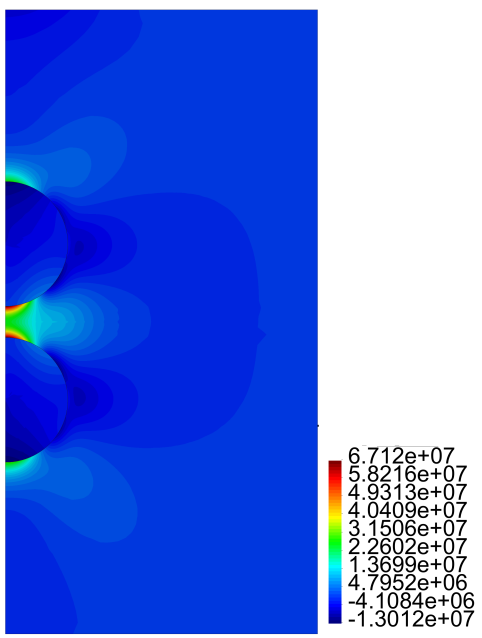

Figure 12. Contour plot for $T_{r r}$ in $\mathrm{Pa}$ for the problem of two inclusions.

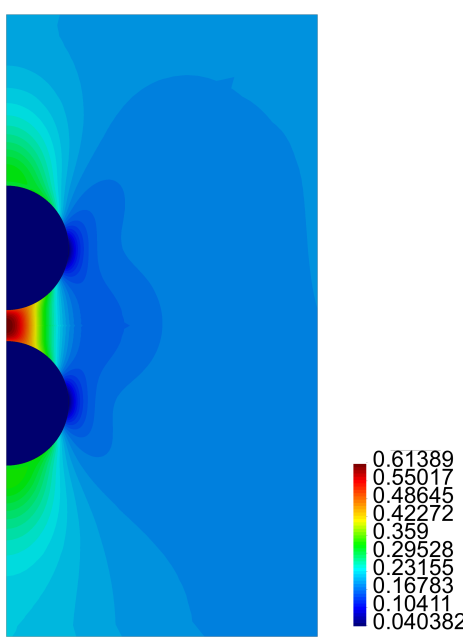

Figure 13. Contour plot for $\varepsilon_{z z}$ for the problem of two inclusions.

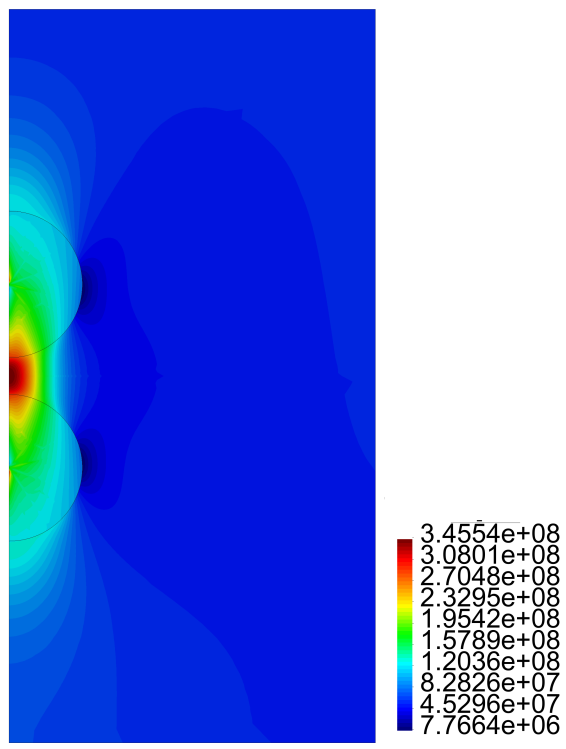

Figure 14. Contour plot for $T_{z z}$ in $\mathrm{Pa}$ for the problem of two inclusions.

\subsection{Results for five inclusions}

Figure 15 presents results for the axial and radial components of the stress (normalized stresses, see (18)) and the strain, for the line $r=0,0 \leq \bar{z} \leq \bar{L}$ for a hyperelastic cylinder with five inclusions (see Figure $3)$.
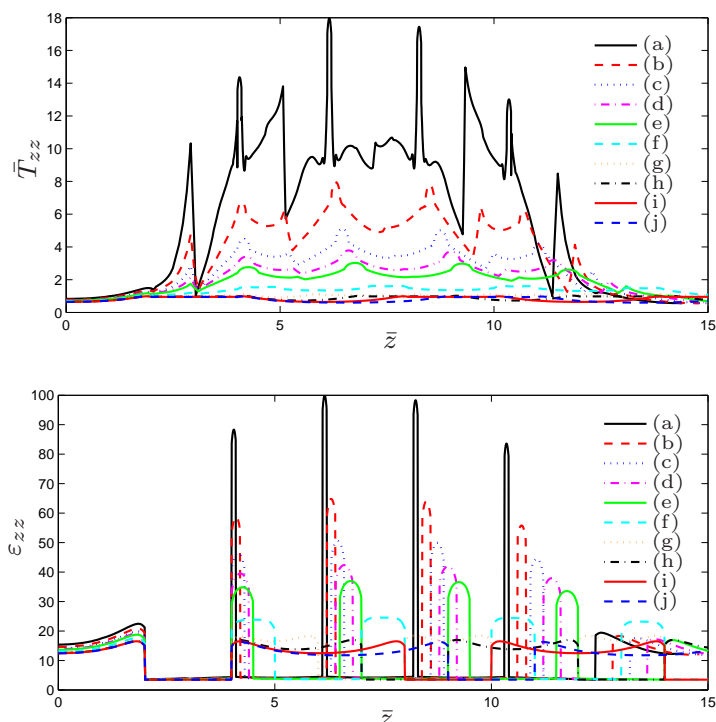

Figure 15. Results for the normalized components of the stress $\bar{T}_{z z}$ and strain $\varepsilon_{z z}$, for the line $r=0,0 \leq \bar{z} \leq \bar{L}$, where (a) $h=2.1 r_{i}$ (b) $h=2.2 r_{i}$ (c) $h=2.3 r_{i}$ (d) $h=2.4 r_{i}$ (e) $h=2.5 r_{i}$ (f) $h=3 r_{i}$ (g) $h=4 r_{i}$ (h) $h=5 r_{i}$ (i) $h=6 r_{i}$ (j) $h=7 r_{i}$.

In Figures 16-19 we present results for the radial and axial components of the strain and the stress, for the case $h=2.5 r_{i}$ (the stresses are in $\mathrm{Pa}$.) 

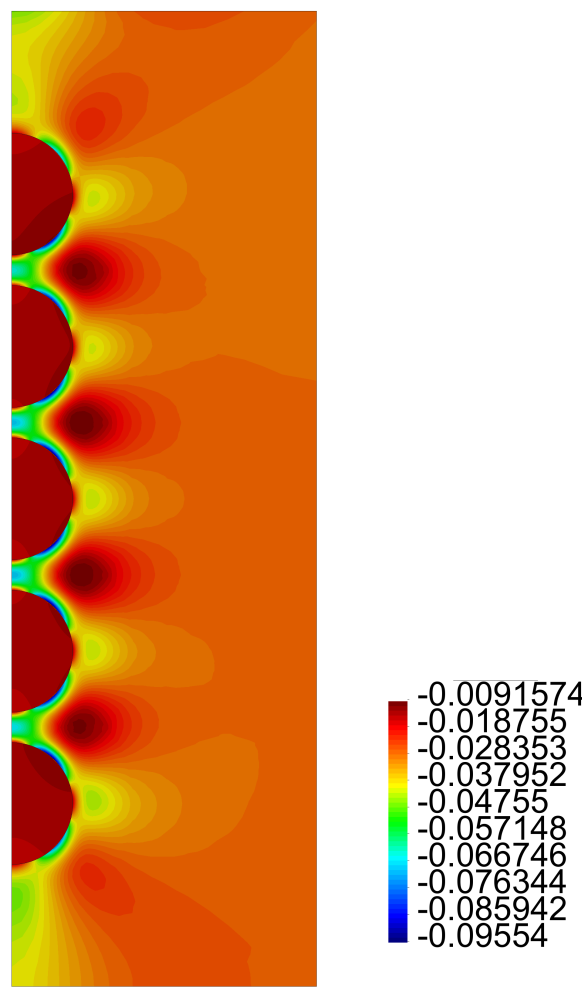

$-0.0035$

$-0.037952$

$-0.03795$

$-0.057148$

$-0.066746$

-0.076344

-0.085942

-0.09554

Figure 16. Contour plot for $\varepsilon_{r r}$ for the problem of five inclusions.
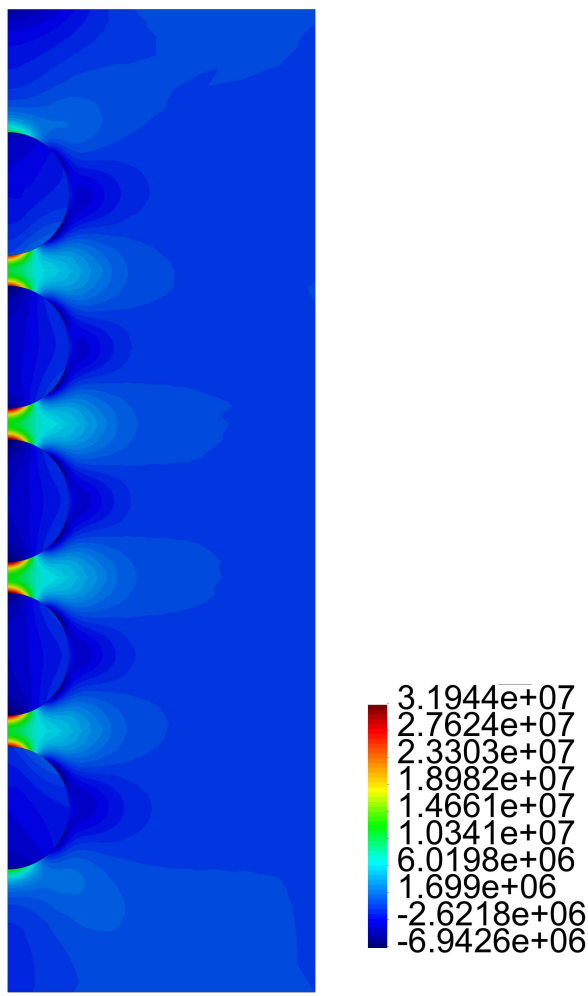

Figure 17. Contour plot for $T_{r r}$ in $\mathrm{Pa}$ for the problem of five inclusions.
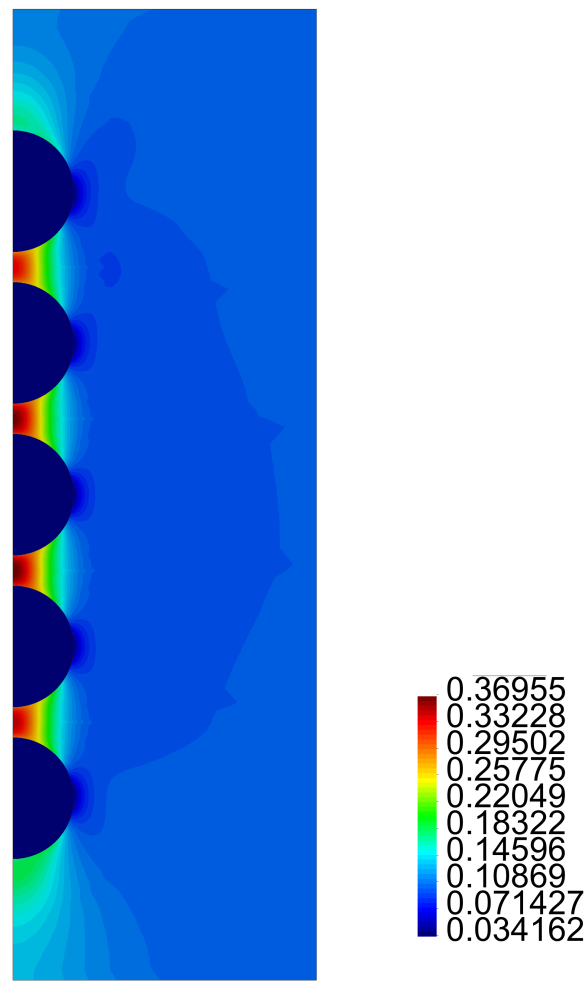

Figure 18. Contour plot for $\varepsilon_{z z}$ for the problem of five inclusions.
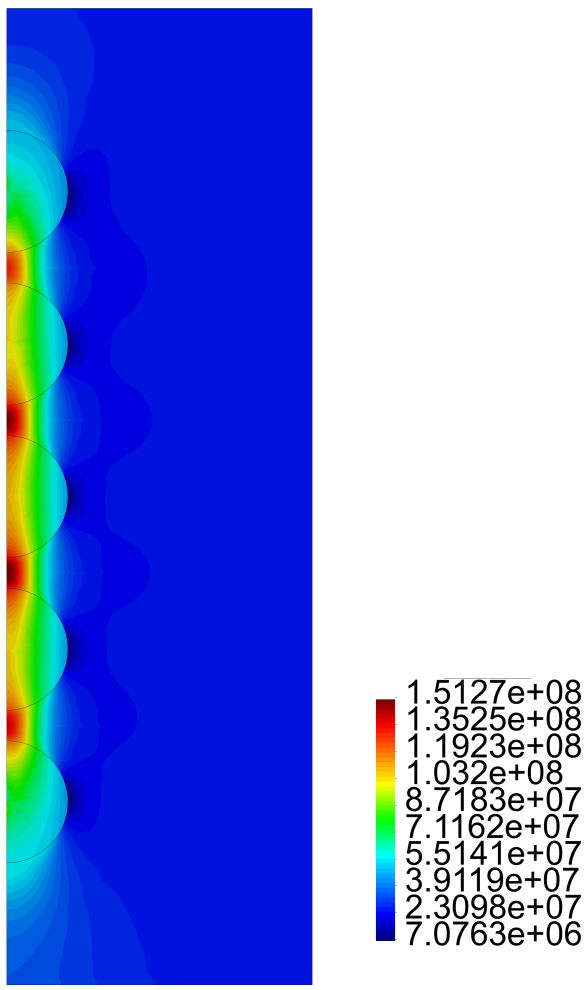

Figure 19. Contour plot for $T_{z z}$ in Pa for the problem of five inclusions. 


\subsection{Discussion of the results}

For the matrix with one particle, from Figure 4 cases (c) and (d), it is observed there is no meaningful difference between the behaviour of the stress and the strain, i.e., as expected for $R$ large enough, the results tend to be invariant of the size of the cylinder. For the results presented in Figure 5, as in the previous case, for $L$ large enough, there is no much difference in the behaviour of the body. From Figures 4 and 5, we observe that the component of the stress are continuous across the surface of the inclusion, but the components of the strain are not. In both cases, it is recognized the presence of large stresses in the matrix material near the interface with the inclusion, and for $T_{z z}$ such stresses are positive, which could eventually lead to debonding of the composite. In fact, in Figure 7 we recognize that there is a zone on the upper part of the spherical inclusion (in the matrix), where the radial stress $T_{r r}$ is positive, and that effect is much stronger in the same zone for $T_{z z}$ (see Figure 9).

For the cylinder with two spherical inclusions, from Figure 10 we observe that there is a considerable difference in the behaviour of the composite if $h$ is varied. Compare for instance the results presented in cases (a), (b) and (d) of that figure, where $h=2.1 r_{i}$, $h=2.2 r_{i}$ and $h=2.3 r_{i}$, respectively. The difference in behaviour between $T_{z z}$ and $\varepsilon_{z z}$ is large (in particular for the case (a) for $T_{z z}$.) In the plot for $\varepsilon_{z z}$, we observe the jump in the value for that component of the strain across the interface between the inclusion and the surrounding matrix. In Figures 12, 14 we notice the large values for $T_{r r}$ and $T_{z z}$ in the matrix, for the zone connecting the two inclusions.

Finally, for the cylinder with five inclusions, as in the previous case, from Figure 15 we notice large values for $\varepsilon_{z z}$ for the matrix material between the inclusions. Also large values and rapid variations for $T_{z z}$ in the same zone are observed, especially for the cases (a), (b) and (c). From Figures 16-19 we observe the same large values for the components of the stress and the strain in the zone near the inclusions.

\section{Concluding remarks}

In the present communication, we have studied the behaviour of a composite consisting of a hyper-elastic matrix with one, two and five spherical inclusions that are modelled using some relatively new classes of constitutive equations, in which, as a particular case such inclusions undergo small strains independently of the magnitude of the stresses. In several of the previous works discussed in the introduction section (see, for example, Refs. $[10,17]$ and the reference mentioned therein), the main idea of studying constitutive equations of the type (10), (13), was to analyse the behaviour of the solutions for problems exhibiting concentration of stresses, where from the physical point of view, it is expected that the strain remain small. This is the case of brittle bodies with cracks (see the discussion in Ref. [14]). In the present work, this has been also the purpose. Herein, problems exhibiting concentration of stresses near the boundary of inclusions were studied. From the results presented in Section 4, it is observed that indeed there exists concentration of stresses, but strains remain small inside the inclusions. The results presented in this paper should be considered as the outcome of a new way to study the problem of modelling the behaviour of composite materials, where there is a soft matrix filled with a relatively stiff and brittle inclusions.

\section{Acknowledgment}

The authors would like to express his gratitude for the financial support provided by FONDECYT (Chile) under grant no. 1120011. The work of S. Montero was also funded by a Scholarship for master degree students provided by CONICYT (Chile).

\section{References}

[1] K. R. Rajagopal, "On implicit constitutive theories," Applications of Mathematics, vol. 48, no. 4, pp. 279-319, 2003. [Online]. Available: https://doi.org/10.1023/A:1026062615145

[2] —_ "The elasticity of elasticity," Zeitschrift für angewandte Mathematik und Physik, vol. 58, no. 2, pp. 309-317, 2007. [Online]. Available: https://doi.org/10.1007/s00033-006-6084-5

[3] K. Rajagopal and A. Srinivasa, "On the response of non-dissipative solids," Proceedings of the Royal Society of London A: Mathematical, Physical and Engineering Sciences, vol. 463, no. 2078, pp. 357-367, 2007. [Online]. Available: https://dx.doi.org/10.1098/rspa.2006.1760

[4] C. Truesdell and W. Noll, The non-linear field theories of mechanics, 3rd ed., S. Antman, Ed. Springer Berlin Heidelberg, 2004. [Online]. Available: https://goo.gl/t8UfwX

[5] K. R. Rajagopal and U. Saravanan, "Extension, inflation and circumferential shearing of an annular cylinder for a class of compressible elastic bodies," Mathematics and Mechanics of Solids, vol. 17, no. 5, pp. 473-499, 2012. [Online]. Available: https://doi.org/10.1177/1081286511423125

[6] R. Bustamante and K. R. Rajagopal, "A nonlinear model for describing the mechanical behaviour of rock," Acta Mechanica, pp. 1-22, 2017. [Online]. Available: https://doi.org/10.1007/s00707-017-1968-3 
[7] P. A. Johnson and P. N. J. Rasolofosaon, "Manifestation of nonlinear elasticity in rock: Convincing evidence over large frequency and strain intervals from laboratory studies," Nonlinear Processes in Geophysics, vol. 3, no. 2, pp. 77-88, 1996. [Online]. Available: https://goo.gl/bnyjUK

[8] Z. Grasley, R. El-Helou, M. D'Ambrosia, D. Mokarem, C. Moen, and K. Rajagopal, "Model of infinitesimal nonlinear elastic response of concrete subjected to uniaxial compression," Journal of Engineering Mechanics, vol. 141, no. 7, p. 04015008, 2015. [Online]. Available: https://dx. doi.org/10.1061/(ASCE)EM.1943-7889.0000938

[9] V. Kulvait, J. Málek, and K. R. Rajagopal, "Modeling gum metal and other newly developed titanium alloys within a new class of constitutive relations for elastic bodies," Archives of Mechanics, vol. 69, no. 3, pp. 223-241, 2017. [Online]. Available: https://goo.gl/qLkpQP

[10] — , "Anti-plane stress state of a plate with a v-notch for a new class of elastic solids," International Journal of Fracture, vol. 179, no. 1-2, pp. 59-73, 2013. [Online]. Available: https://doi.org/10.1007/s10704-012-9772-5

[11] M. L. Williams, "On the stress distribution at the base of a stationary crack," Journal of Applied Mechanics, vol. 24, no. 1, pp. 109-114, 1956. [Online]. Available: https://goo.gl/q9uZUn

[12] H. Zhu, A. Muliana, and K. Rajagopal, "On the nonlinear viscoelastic deformations of composites with prestressed inclusions," Composite Structures, vol. 149, Supplement C, pp. 279-291, 2016. [Online]. Available: https: //doi.org/10.1016/j.compstruct.2016.03.008

[13] C. Truesdell and R. Toupin, The classical field theories. Springer, Berlin, Heidelberg: Principles of Classical Mechanics and Field Theory / Prinzipien der Klassischen Mechanik und Feldtheorie. Encyclopedia of Physics / Handbuch der Physik, 1960, vol. 2/3/1, pp. 226-858. [Online]. Available: https://doi.org/10.1007/978-3-642-45943-6_2

[14] K. R. Rajagopal, "On the nonlinear elastic response of bodies in the small strain range," Acta Mechanica, vol. 225, no. 6, pp. 1545-1553, 2014. [Online]. Available: https://doi.org/10.1007/s00707-013-1015-y

[15] R. Bustamante and K. R. Rajagopal, "A note on plane strain and plane stress problems for a new class of elastic bodies," Mathematics and Mechanics of Solids, vol. 15, no. 2, pp. 229-238, 2010. [Online]. Available: https://doi.org/10.1177/1081286508098178

[16] A. Ortiz-Bernardin, R. Bustamante, and K. Rajagopal, "A numerical study of elastic bodies that are described by constitutive equations that exhibit limited strains," International Journal of Solids and Structures, vol. 51, no. 3, pp. 875-885, 2014. [Online]. Available: https://doi.org/10.1016/j.ijsolstr.2013.11.014

[17] S. Montero, R. Bustamante, and A. OrtizBernardin, "A finite element analysis of some boundary value problems for a new type of constitutive relation for elastic bodies," Acta Mechanica, vol. 227 , no. 2, pp. 601-615, 2016. [Online]. Available: https://doi.org/10.1007/s00707-015-1480-6 


\title{
COMPARACIÓN ENTRE REDES NEURONALES ARTIFICIALES Y REGRESIÓN MÚLTIPLE PARA LA PREDICCIÓN DE LA RUGOSIDAD SUPERFICIAL EN EL TORNEADO EN SECO
} Comparison Between Artificial Neural Network And Multiple Regression For The Prediction of Superficial Roughness In DRY TURNING

\author{
Yoandrys Morales-Tamayo ${ }^{1, *}$, Yusimit Zamora-Hernández ${ }^{2}$, Paco Vásquez-Carrera ${ }^{1}$, \\ Mario Porras-Vásconez ${ }^{1}$, Joao Bárzaga-Quesada ${ }^{3}$, Ringo López-Bustamante ${ }^{4}$
}

\section{Resumen}

Los métodos de regresión múltiple y redes neuronales artificiales son técnicas usadas en muchas aplicaciones de la industria. En este trabajo se utilizaron dos métodos de predicción: regresión múltiple y redes neuronales artificiales (perceptrón multicapa) con el objetivo de predecir la rugosidad superficial en el torneado en seco del acero AISI 3161. En su implementación fueron considerados varios parámetros de corte como la velocidad, el avance y el tiempo de mecanizado. Las ecuaciones obtenidas por ambos métodos fueron comparadas desarrollando un diseño factorial completo para aumentar la fiabilidad de los valores registrados de rugosidad superficial. En el análisis se puede comprobar mediante los valores de coeficientes de determinación que los modelos propuestos son capaces de predecir la rugosidad superficial. Los modelos obtenidos demuestran que la técnica de redes neuronales artificiales tiene mejor precisión que la regresión múltiple para este estudio.

Palabras clave: acero inoxidable AISI 316L, análisis de varianza y regresión, redes neuronales artificiales, rugosidad superficial, torneado de alta velocidad.

\section{Abstract}

The simple regression and artificial neural network methods are techniques used in many industrial aplications. This work developed two models in order to predict the surface roughness in dry turning of AISI 316L stainless steel. In its implementation they were considered various cutting parameters such as cutting speed, feed, and machining time. The models obtained by both methods were compared to develop a full factorial design to increase reliability of the recorded values of roughness. The analysis can be corroborated by the values of coefficients of determination that the proposed models are able to predict for surface roughness. The obtained results show that the neural networks techniques are more accurate than the multiple regression techniques for this study.

Keywords: AISI 316L stainless steel, Analysis of variance and regression, Artificial neural network, Dry high-speed turning, Surface roughness.

\footnotetext{
$\overline{1, *}$ Dirección en Electromecánica, Universidad Técnica de Cotopaxi, Cotopaxi - Ecuador. Autor para correspondencia yoandrys.morales@utc.edu.ec, (D) http://orcid.org/0000-0001-7456-1490, (D) http://orcid.org/0000-0003-4734-8584, (D) http://orcid.org/0000-0002-4119-4812

${ }^{2}$ Departamento de Ingeniería Mecánica, Universidad de Granma, Cuba. (1) http://orcid.org/0000-0002-0112-1061

${ }^{3}$ Departamento de Ciencias de la Energía y Mecánica, Universidad de las Fuerzas Armadas, Cotopaxi - Ecuador. (D) http://orcid.org/0000-0001-9792-7379

${ }^{4}$ Dirección de Extensión La Maná, Universidad Técnica de Cotopaxi, Ecuador. (D) http://orcid.org/0000-0002-6519-1587

Recibido: 30-11-2017, aprobado tras revisión: 18-12-2017

Forma sugerida de citación: Morales, Y.; Zamora, Y.; Vásquez, P.; Porras, M.; Bárzaga, J.; López, R. (2018). «Comparación entre redes neuronales artificiales y regresión múltiple para la predicción de la rugosidad superficial en el torneado en seco». Ingenius. N. ${ }^{\circ} 19$, (enero-junio). pp. 79-88. DOI: https://doi.org/10.17163/ings.n19.2018.08.
} 


\section{Introducción}

El acero inoxidable es uno de los materiales metálicos más utilizados en el sector industrial, esto se debe a la favorable combinación de las propiedades mecánicas, resistencia a la corrosión y costo. Este material ha sido ampliamente usado en el campo aeroespacial y militar, donde existe una demanda creciente en los requisitos de calidad superficial [1].

Las características de la superficie mecanizada afectan directamente la resistencia a la fatiga, la resistencia a la corrosión y las propiedades tribológicas de los componentes mecanizados. Obtener un valor elevado de calidad superficial aumenta la vida a la fatiga del producto. En consecuencia, el control de la superficie mecanizada es esencial para asegurar un correcto proceso de corte. El aspecto más importante en los procesos de manufactura es la medición y la caracterización de las propiedades superficiales. En el proceso de torneado, la rugosidad superficial es un parámetro que tiene gran influencia en el comportamiento y la funcionalidad de los componentes mecánicos y en los costos de producción [2].

La rugosidad superficial es afectada por varios factores, tales como: el avance, las propiedades del material de trabajo, la velocidad de corte, la profundidad, el radio de la punta, las condiciones de la máquina, los fluidos de corte, los materiales de las herramientas de corte y los ángulos de la herramienta de corte, entre otros. Dentro de ellos, son fáciles de ajustar los parámetros de corte con el objetivo de lograr el rendimiento esperado [3].

Los aceros inoxidables austeníticos son considerados materiales difíciles de mecanizar, característica relacionada con su baja conductividad térmica, alto coeficiente de expansión térmica, alta ductilidad y alto endurecimiento por deformación. Las operaciones de acabado efectuadas en estos aceros son comúnmente ejecutadas con insertos de carburos recubiertos. El rango de las velocidades recomendadas para el torneado de estos aceros son muy conservadoras (200-350 m/min) [4].

La utilización de bajas velocidades de corte, conduce a una baja eficiencia en la producción y consecuentemente altos costos de producción [5]. Al ser este rango improductivo en las condiciones actuales de la tecnología, es necesario determinar el comportamiento de la rugosidad superficial durante el proceso de mecanizado de alta velocidad (HSM).

La rugosidad superficial generada en los procesos de mecanizado ha sido estudiada desde [6] por Sata y en [7] por Dickinson. El efecto del avance de la herramienta, del radio de la punta y del ángulo del filo sobre la rugosidad superficial generada en el torneado fue descrita por Groover y nombrada «rugosidad ideal», enunciada como la mínima rugosidad que se genera en una pieza torneada [8].
La rugosidad superficial es uno de los parámetros de calidad más estudiado por los investigadores que analizan la maquinabilidad de los aceros austeníticos. Por ejemplo, Korkut y colaboradores realizaron un estudio de la rugosidad superficial y el desgaste del flanco para determinar la velocidad de corte optima con el uso de insertos de carburos recubiertos. Los mayores valores de rugosidad superficial durante el torneado se alcanzaron a bajas velocidades $(<180 \mathrm{~m} / \mathrm{min})$ atribuido a la presencia del crecimiento del filo de corte [9]. Similar resultado fue alcanzado por Ciftci durante su estudio experimental para analizar la influencia de la velocidad de corte (entre $120 \mathrm{~m} / \mathrm{min}$ y $210 \mathrm{~m} / \mathrm{min}$ ) sobre la rugosidad superficial y las fuerzas de corte [9].

Cuatro años más tarde, Galanis y Manolakos desarrollaron un modelo matemático empírico para predecir la rugosidad superficial con la aplicación de una metodología de superficie de respuesta. Esta investigación fue desarrollada durante el mecanizado de cabezas femorales con una herramienta recubierta de $\left(\mathrm{TiN} / \mathrm{Al}_{2} \mathrm{O}_{3} / \mathrm{TiC}\right)[10]$.

En el 2012, Çaydaş y Ekici implementaron una red neuronal artificial para predecir la rugosidad superficial. La validación de este modelo fue desarrollada mediante un estudio experimental que consideró los parámetros de corte involucrados en el torneado en seco del acero inoxidable AISI 304 [11].

Ese mismo año, Ahilan y otros realizaron una investigación con el propósito de desarrollar un modelo basado en redes neuronales artificiales para predecir las condiciones de corte en tornos CNC. Ellos utilizaron el diseño de experimentos (método Taguchi) para entrenar y validar el modelo neuronal propuesto [12]. En este caso la velocidad de corte máxima utilizada fue $150 \mathrm{~m} / \mathrm{min}$.

Selvaraj y otros, desarrollaron una investigación para optimizar los parámetros de corte con el objetivo de minimizar la rugosidad superficial, la fuerza de corte y el desgaste de la herramienta. Los experimentos son analizados usando el método Taguchi, la operación de torneado fue ejecutada en seco y a una velocidad de corte máxima de $120 \mathrm{~m} / \mathrm{min}$ [13].

En la Figura 1 se puede observar un resumen de las velocidades de corte utilizadas en los principales estudios desarrollados durante el torneado de los aceros austeníticos. Estas investigaciones incluyen no solo el estudio de la rugosidad superficial, sino también investigaciones de desgaste de las herramientas de corte, integridad superficial, fuerzas de corte, potencia de corte y formación de la viruta.

La literatura revela (Figura 1) que existen pocos estudios relacionados con el torneado en seco de los aceros inoxidables austeníticos a velocidades de corte superiores a los $400 \mathrm{~m} / \mathrm{min}$. Solo cuatro autores sobrepasaron esta velocidad de corte.

Lin evalúa el comportamiento del desgaste de la he- 
rramienta (desgaste del flanco) [14], Fernández-Abia y colaboradores no realizaron el mecanizado en seco [15], Maranhão y Darvim estudiaron la influencia del coeficiente de fricción de la interface herramienta-viruta [16] y por último, Galanis y Manolakos estudiaron el efecto de las condiciones de corte en la rugosidad superficial durante el mecanizado de cabezas femorales de acero inoxidable AISI 316L, para este estudio la longitud de corte fue de $28 \mathrm{~mm}$ [17].

El objetivo de este trabajo es comparar dos métodos para predecir la rugosidad superficial en el acero inoxidable AISI 316L con velocidades de $400 \mathrm{~m} / \mathrm{min}$ y $450 \mathrm{~m} / \mathrm{min}$, uno basado en regresión múltiple y otro en las redes neuronales artificiales del tipo perceptrón multicapa.

Para este propósito fue preciso implementar un diseño factorial completo para investigar el efecto de las condiciones de corte (velocidad, avance, tiempo) en la rugosidad superficial. Los modelos de regresión múltiple son validados mediante los supuestos básicos.

Una arquitectura de perceptrón multicapa con algoritmo back-propagation es usado para desarrollar la red neuronal y el criterio para la actualización de los pesos es gradiente descendente. La efectividad de ambos modelos se determina comparando los coeficientes de determinaciones y el error medio absoluto.

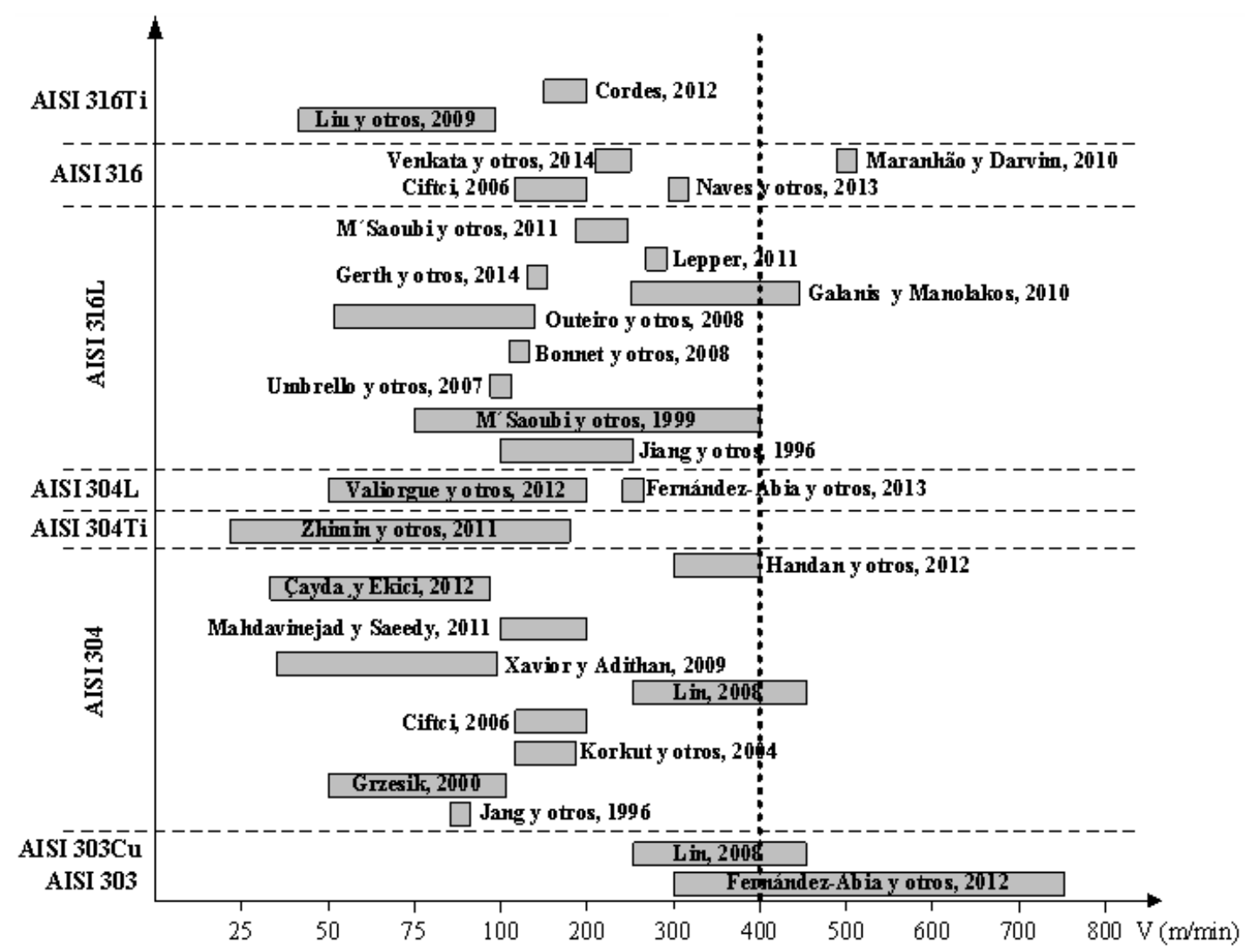

Figura 1. Principales investigaciones en el torneado de los aceros inoxidables austeníticos.

\section{Materiales y métodos}

\subsection{Modelos de rugosidad superficial}

En el torneado existen muchos factores que afectan la rugosidad superficial como son la herramienta de corte, el material de trabajo y los parámetros de corte. Los factores relacionados con las herramientas son el material, el radio de la punta, el ángulo de ataque, la geometría de la arista de corte, la vibración de la herramienta, etc., mientras que entre las variables relacionadas con el material de la pieza de trabajo se tiene la dureza, las propiedades físicas y mecánicas, entre otras. Por otro lado, las condiciones de corte que influyen son la velocidad de corte, el avance y la profundidad [18].

La selección adecuada de los parámetros de corte y de la geometría de la herramienta resulta compleja y difícil para lograr la calidad superficial requerida [19]. Por tanto, está claro que la selección y obtención de un modelo que describa este proceso es esencial para el mecanizado de los aceros [20].

La rugosidad superficial ( $\mathrm{Ra}$ ) es generalmente definida basada en la Norma ISO 4287 como la media aritmética de la desviación del perfil de la rugosidad 
desde la línea central a lo largo de la medición. Esta definición es dada en la ecuación (1).

$$
R_{a}=\frac{1}{L} \int_{0}^{L}|y(x)| d x
$$

Donde: $L$ es la longitud de medición; $y$ es la distancia entre dos puntos del perfil. La relación entre la rugosidad superficial y las variables de mecanizado puede ser definida como (ecuación 2):

$$
R_{a}=C \cdot V^{n} \cdot f^{m} \cdot d^{p} \cdot r^{l} \cdot \varepsilon
$$

Donde, $R a$ es la rugosidad superficial medida en $\mu \mathrm{m} ; V, f, d, r$ son velocidad de corte $(\mathrm{m} / \mathrm{min})$, avance $(\mathrm{mm} / \mathrm{rev})$, profundidad $(\mathrm{mm})$, radio de la punta de la herramienta $(\mathrm{mm})$, respectivamente. $C, m, n, l$ son constantes y $\varepsilon$ es el error aleatorio [21]. La ecuación (1) puede ser vista como la ecuación (3) para facilitar la representación de las constantes y los parámetros. La rugosidad media aritmética $(\mathrm{Ra})$ y la altura del pico máximo (Rt) de las superficies torneadas pueden ser determinadas por las ecuaciones siguientes (3) y (4):

$$
\begin{aligned}
R_{a} & \approx \frac{f^{2}}{32 \cdot r} \\
R_{t} & \approx \frac{f^{2}}{8 \cdot r}
\end{aligned}
$$

Donde $r$, es el radio de la punta $(\mathrm{mm})$ y $f$, es el avance de corte $(\mathrm{mm} / \mathrm{rev})$. Las ecuaciones (3) y (4) muestran que la rugosidad superficial incrementa proporcionalmente con el avance y, además, el aumento del radio de la punta de la herramienta de corte reduce la rugosidad superficial en el torneado.

\subsection{Modelación por regresión múltiple}

La regresión múltiple es una técnica estadística que permite determinar la correlación que existe entre variables independientes y dos o más variables dependientes. La regresión múltiple se puede utilizar para analizar datos ordinales y categóricos [22]. Por lo general, se realiza primeramente un análisis de varianza (Anova) para determinar los factores importantes involucrados y luego con el uso de la regresión se obtiene un modelo cuantitativo que relaciona los factores más importantes con la respuesta [23].

\subsection{Estrategia de predicción usando redes neu- ronales artificiales}

Las redes neuronales artificiales (RNA) son ampliamente usadas en muchas aplicaciones de la industria. Estas son muy populares en la modelación de sistemas debido a su alta eficiencia en la adaptación y en el aprendizaje mediante el reconocimiento de patrones [3].
La red instalada en esta investigación es una red perceptrónica multicapa la cual corresponde en equivalencia a la regresión no lineal múltiple [24]. La red perceptrónica multicapa está compuesta por la asociación de neuronas artificiales organizadas dentro de la red formando niveles o capas.

En este caso corresponde una capa de entrada en la que se introducen los patrones en la red (parámetros de corte), una capa oculta con algunas neuronas y una capa de salida con la variable respuesta (rugosidad superficial). La estructura de la RNA que se muestra en Figura 2 fue utilizada para modelar y predecir la variable dependiente.

La determinación del número óptimo de neuronas de la capa oculta se realizó mediante un proceso de ensayo y error en el que se probaron diferentes variantes. En todo caso, el objetivo fue dotar a la red de un número adecuado de neuronas en la capa oculta para garantizar la capacidad de aprendizaje de las características de las posibles relaciones existentes entre los datos de la muestra.

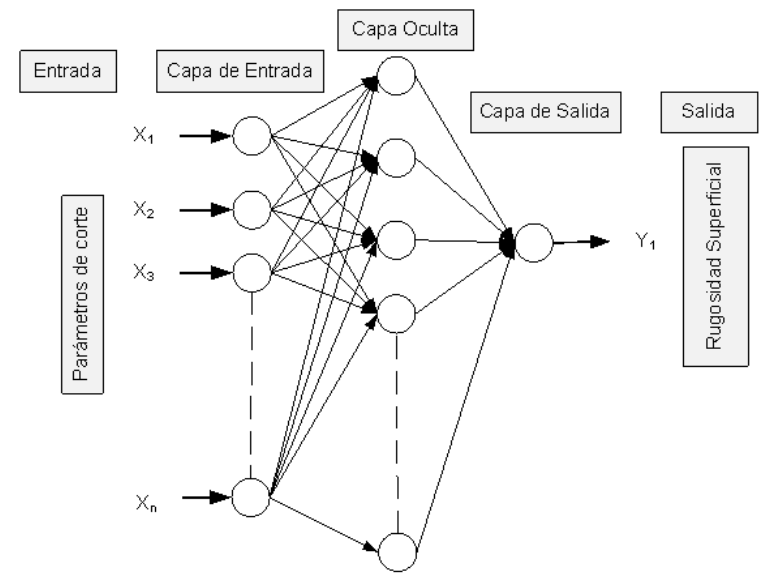

Figura 2. Estructura de la red perceptrónica multicapa

\subsection{Ensayos experimentales}

El torneado experimental fue ejecutado en condiciones secas, con el uso del torno multifuncional tipo Okuma Multus B200-W con potencia del motor de $15 \mathrm{~kW}$ y rotación del husillo entre $50 \mathrm{rpm}$ y $5000 \mathrm{rpm}$ (Figura $3)$.

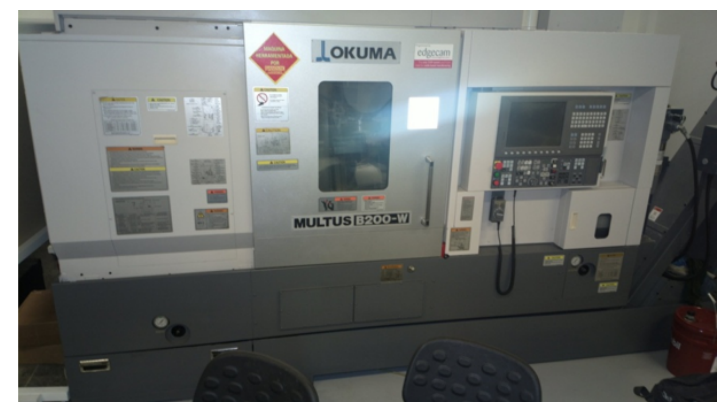

Figura 3. Torno multifuncional CNC marca Okuma modelo Multus B-200W 
El acero inoxidable AISI 316L fue el material seleccionado para las probetas, este acero es utilizado en la fabricación de productos resistentes a la corrosión y resistentes a altas temperaturas [25]. La composición química es C $0,015 \%$, Si $0,58 \%$, Mn 1,50\%, Cr 16,95 $\%$, Mo 2,05\%, Ni 10,08 \%, P 0,031 \%, S 0,029\% y N $0,059 \%$.

Las probetas de $100 \mathrm{~mm}$ de diámetro y $200 \mathrm{~mm}$ de longitud fueron torneadas con insertos recubiertos con calidad Sandvik, GC1115 y GC2015. Los recubrimientos de $\left(\mathrm{TiCN}-\mathrm{Al}_{2} \mathrm{O}_{3}-\mathrm{TiN}\right)$ con un espesor de $15 \mu \mathrm{m}$ correspondieron al inserto GC2015 y para el inserto tipo GC1115 su recubrimiento fue de TiN con $5 \mu \mathrm{m}$ de espesor. Después de la operación de torneado, la rugosidad superficial (Ra) fue medida mediante un rugosímetro CARL ZEISS modelo SURFCOM 1500SD2 (Figura 4).

La geometría de los insertos fue CCMT 1204 04-MF con rompevirutas, el portaherramienta marca Sandvik de código C6-SCLCL-45065-12 y un adaptador con código C6-391.01-63 060. El ángulo de incidencia principal fue de $7^{\circ}$, el ángulo de ataque de $0^{\circ}$ y el radio de la punta de $0,4 \mathrm{~mm}$.

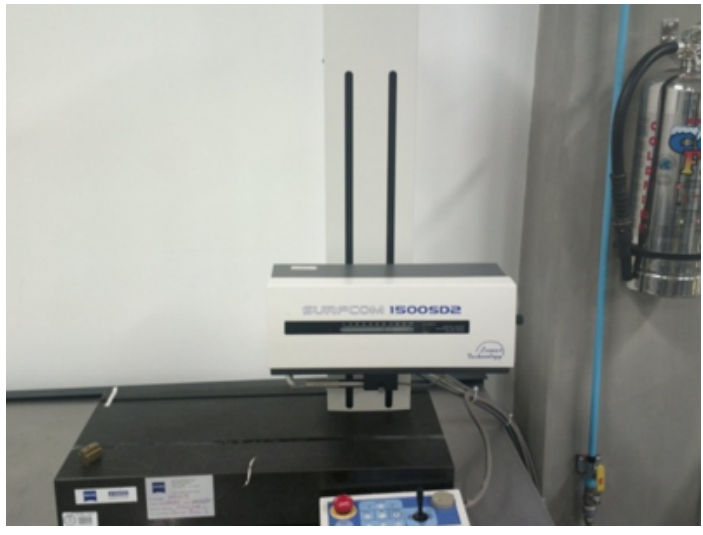

Figura 4. Rugosímetro CARL ZEISS modelo SURFCOM 1500SD2.

$\mathrm{El}$ análisis factorial completo fue el procedimiento utilizado para determinar la relación entre las variables independientes (parámetros de corte) y la variable dependiente (rugosidad superficial $(R a)$ ). Un total de 64 ensayos para dos réplicas fueron desarrollados con dos niveles de velocidades de corte $(v)$, cuatro niveles de tiempo $(T)$, dos niveles de avances de corte $(f)$ y dos niveles de material de la herramienta, en la Tabla 1 aparecen las variables estudiadas.

Tabla 1. Factores y niveles utilizados en el desarrollo del experimento

\begin{tabular}{|c|c|c|c|c|c|c|}
\hline Factores & & Símbolos & Nivel 1 & Nivel 2 & Nivel 3 & Nivel 4 \\
\hline Avance (mm/rev) & & $\mathrm{f}$ & 0,08 & 0,16 & - & - \\
\hline Material inserto & & Ins & GC1115 & GC2015 & - & - \\
\hline Velocidad $(\mathrm{m} / \mathrm{min})$ & & $\mathrm{V}$ & 400 & 450 & - & - \\
\hline \multirow{2}{*}{ Tiempo (min) } & \multirow{2}{*}{$\mathrm{T}$} & $400 \mathrm{~m} / \mathrm{min}$ & 2 & 3 & 4 & 5 \\
\hline & & $450 \mathrm{~m} / \mathrm{min}$ & 0,6 & 1,2 & 2 & 3 \\
\hline
\end{tabular}

\section{Resultados y discusión}

La rugosidad superficial es ampliamente utilizada como parámetro para indicar la calidad de un producto y en la mayoría de los casos, un requisito técnico importante en el diseño mecánico.

En consecuencia, lograr la calidad de la superficie deseada es de mucha importancia para el comportamiento funcional de un producto [26]. También tiene un impacto en las propiedades mecánicas, específicamente en la resistencia a la fatiga y en la resistencia a la corrosión [19].

Las industrias manufactureras son las encargadas de garantizar al consumidor las demandas crecientes en la calidad superficial y a su vez en obtener productos menos costosos. Por tanto, conocer el efecto de estos parámetros es importante para evaluar la efectividad y productividad del proceso de corte [27].
En esta sección serán comparados y discutidos los resultados obtenidos mediante regresión múltiple y por redes neuronales artificiales.

\subsection{Análisis mediante regresión múltiple}

Los modelos obtenidos como resultado del análisis de regresión múltiple con la velocidad de $400 \mathrm{~m} / \mathrm{min}$ se muestran en las ecuaciones (5) y (6) para los insertos GC1115 y GC2015 respectivamente. Los modelos con la velocidad de $450 \mathrm{~m} / \mathrm{min}$ se muestran en las ecuaciones (7) y (8) para los insertos GC1115 y GC2015 respectivamente.

$$
\begin{gathered}
R_{a}=0,358933+0,0188793 \cdot e^{T} \cdot f \\
R_{a}=0,27529+0,0109446 \cdot T^{2}+0,69 \cdot f \\
R_{a}=-2,75967+2,99435 \cdot e^{\left(T^{3} \cdot f^{2}\right)}
\end{gathered}
$$




$$
R_{a}=0,219579+0,0201327 \cdot T^{3}+0,45625 \cdot f
$$

El coeficiente de determinación $\left(\mathrm{R}^{2}\right)$ representa la correcta medida de la bondad del ajuste en la línea de regresión determinada por el modelo. Para estos casos los $\mathrm{R}^{2}$ fueron en la velocidad de $400 \mathrm{~m} / \mathrm{min}, 0,92$ (GC1115) y 0,80 (GC2015) y para $450 \mathrm{~m} / \mathrm{min}$ fueron 0,97 (GC1115) y 0,97 (GC2015).

En todos los casos, con el objetivo de validar los resultados de regresión obtenidos se comprobó el cumplimiento de los supuestos básicos de regresión como: la homocedasticidad (Test White), la no autocorrelación de los residuos (Test Breusch-Godfrey), la normalidad ((Test Jarque-Bera) y media nula.

En las Figuras 5 y 6 se muestran las comparaciones entre los valores medidos de manera experimental y los valores estimados de la rugosidad superficial por los modelos correspondientes a las velocidades de $400 \mathrm{~m} / \mathrm{min}$ y $450 \mathrm{~m} / \mathrm{min}$, respectivamente. En estas Figuras se puede observar una fuerte relación entre las variables estimadas y la variable respuesta.

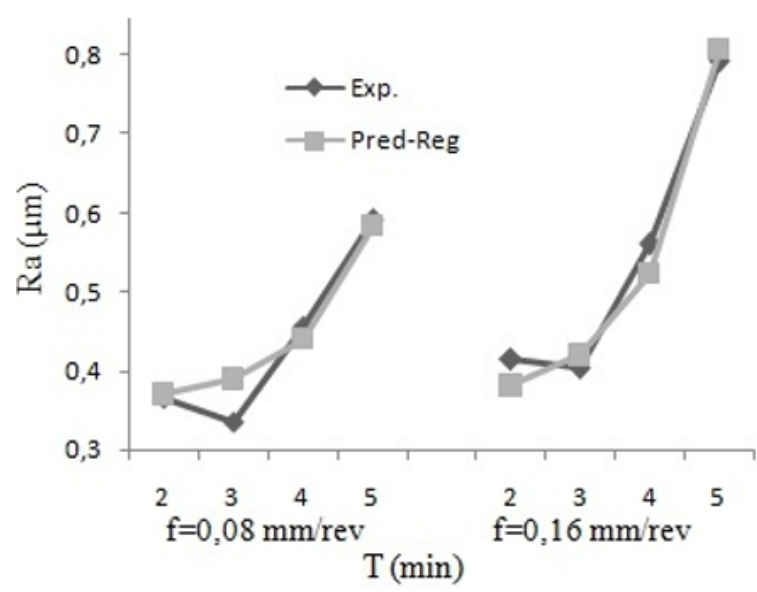

(a)

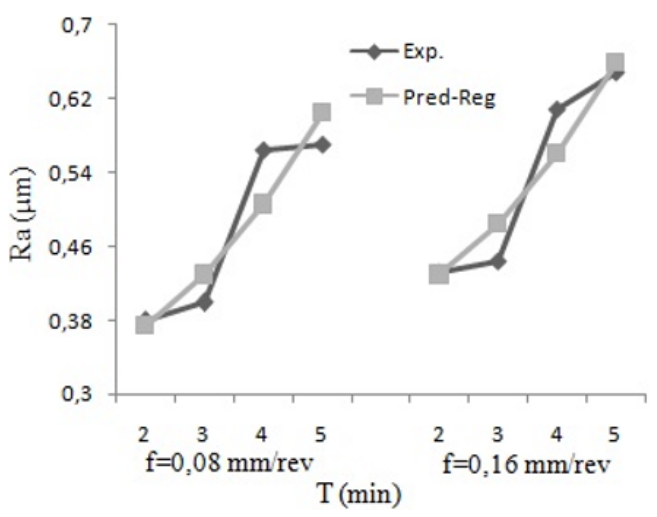

(b)

Figura 5. Valores medidos y estimados por regresión múltiple para $\mathrm{v}=400 \mathrm{~m} / \mathrm{min}$, a) inserto GC1115 y b) inserto GC2015.

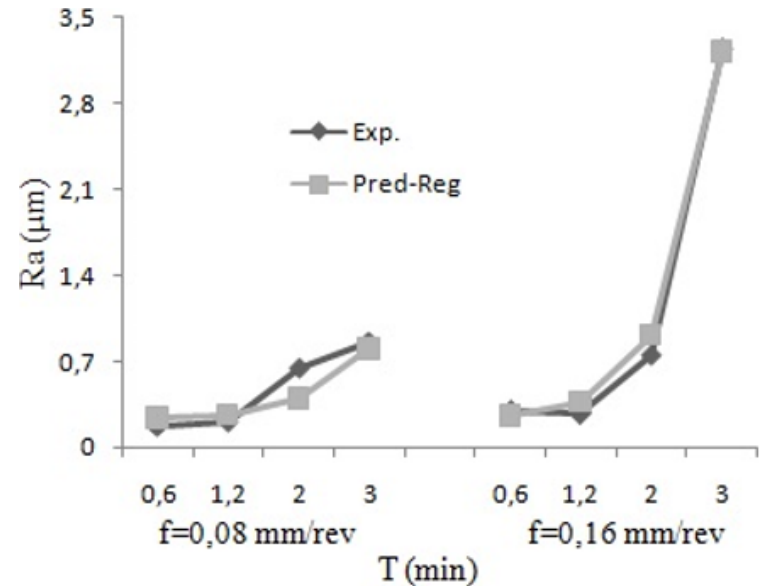

(a)

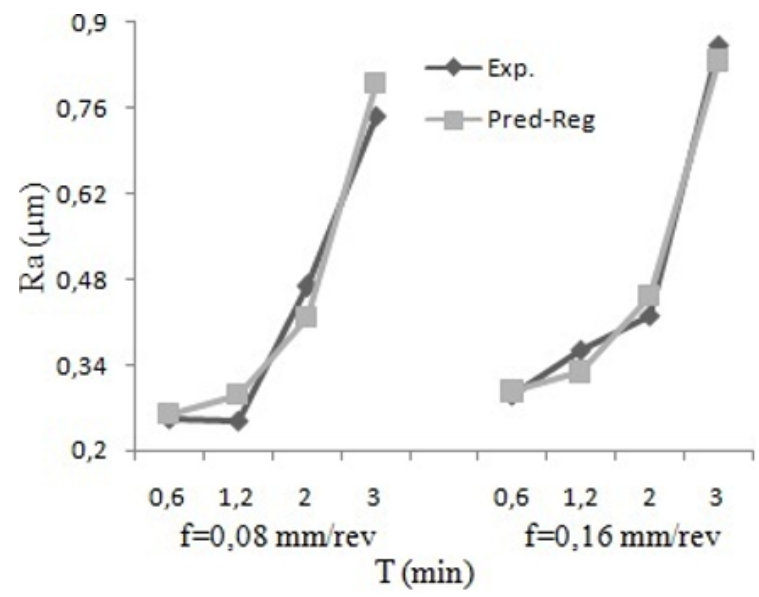

(b)

Figura 6. Valores medidos y estimados por regresión múltiple para $\mathrm{v}=450 \mathrm{~m} / \mathrm{min}$, a) inserto GC1115 y b) inserto GC2015.

\subsection{Resultados de las redes neuronales artifi- ciales}

La estructura de la red aplicada para modelar y predecir la rugosidad superficial en la operación de torneado corresponde al perceptrón multicapa del tipo feedforward Backpropagations. Los datos experimentales fueron utilizados para construir el modelo de las redes neuronales artificiales.

El entrenamiento fue desarrollado a través del algoritmo Levenberg Marquardt. Los mejores resultados fueron obtenidos con la estructura 3-5-1, tres neuronas en la capa de entrada, 5 neuronas en la capa oculta y una en la capa de salida. El software de redes neuronales fue codificado utilizando el Neural Networks Toolbox de Matlab. Los parámetros de la estructura de la red propuesta son mostrados en la Tabla 2.

Los datos de entrada fueron divididos por las velocidades, por tanto, solo se consideraron el tiempo de maquinado, el avance de corte y el tipo de herramienta 
de corte. Estos datos fueron distribuidos aleatoriamente de la siguiente forma, para el entrenamiento fueron seleccionados el $70 \%$ (22 datos), $15 \%$ (5 datos) para la etapa de prueba y para la validación el restante $15 \%$ (5 datos).

Tabla 2. Parámetros de la red neuronal artificial implementada en el estudio

\begin{tabular}{cc}
\hline Número de capas & 3 \\
& Entrada: 3 \\
Número de neuronas en las capas & Oculta: 5 \\
& Salida: 1 \\
Función de activación & Tansig-purelin \\
Número de iteraciones & 10000 \\
\hline
\end{tabular}

Los resultados obtenidos fueron analizados por métodos estadísticos, los criterios utilizados fueron el error medio absoluto (Emedio, (\%)) y el coeficiente de determinación $\left(\mathrm{R}^{2}\right)$.

Las ecuaciones 9 y 10 son utilizadas para calcular estos criterios respectivamente.

$$
\begin{aligned}
E_{\text {medio }} & =\left(\frac{1}{N} \sum_{i}\left|\frac{t_{i}-t_{0}}{t_{i}}\right| \times 100\right) \\
R^{2} & =1-\left(\frac{\sum_{i}\left(t_{i}-t_{0}\right)^{2}}{\sum_{i}\left(t_{0}\right)^{2}}\right)
\end{aligned}
$$

Donde $N$, es el número de ensayos; $t_{i}$, valores experimentales y $t_{0}$, valores estimados.

En las Figuras 7 y 8 se puede observar una comparación entre los valores experimentales y los valores estimados de la rugosidad superficial por el modelo desarrollado mediante redes neuronales artificiales.

Los resultados de las redes neuronales demuestran que los modelos propuestos en este estudio son adecuados para la predicción de la rugosidad superficial.

Los valores de los coeficientes de determinación y de los errores medios absolutos están en los rangos aceptables (Tabla 3).

Tabla 3. Valores de coeficientes de determinación $\left(R^{2}\right)$ y errores medios absolutos para cada red neuronal desarrollada.

\begin{tabular}{ccc}
\hline \multirow{2}{*}{ Parámetro } & \multicolumn{2}{c}{ Red neuronal } \\
& $\mathbf{4 0 0} \mathbf{~} / \mathbf{m i n}$ & $\mathbf{4 5 0} \mathbf{~} / \mathbf{m i n}$ \\
\hline Entrenamiento & 0,98134 & 0,99836 \\
Prueba & 0,99842 & 0,99026 \\
General & 0,98122 & 0,9973 \\
E $_{\text {medio }}$ & 2,869 & 6,946 \\
\hline
\end{tabular}

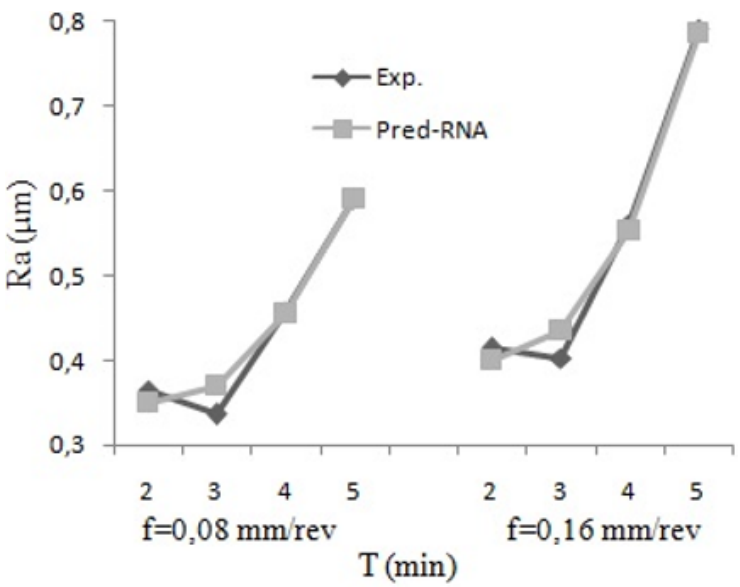

(a)

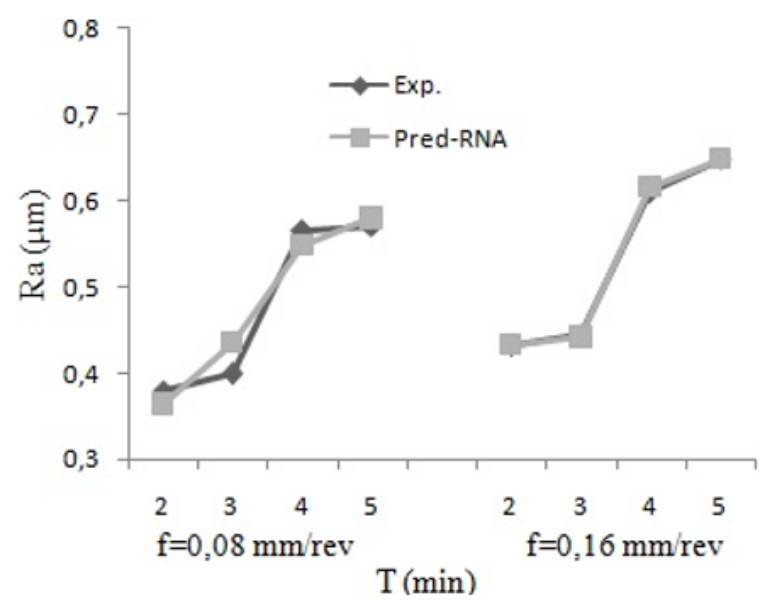

(b)

Figura 7. Valores medidos y estimados por redes neuronales artificiales para $\mathrm{v}=400 \mathrm{~m} / \mathrm{min}$, a) inserto GC1115 y b) inserto GC2015.

\subsection{Evaluación general}

Un diseño de experimento factorial completo fue aplicado para determinar los efectos de las variables independientes (velocidad, avance, tiempo y herramientas de corte) del proceso de torneado en seco en la rugosidad superficial. Después de cada ensayo de torneado, los valores de rugosidad superficial fueron registrados para su posterior análisis. En esta investigación fueron desarrollados modelos mediante redes neuronales artificiales y por regresión múltiple. La Tabla 4 muestra una comparación de los resultados según la precisión de los valores obtenidos mediante regresión múltiple y por redes neuronales artificiales. Los resultados se encuentran cercanos a los medidos de manera experimental para todos los modelos. Por tanto, los modelos propuestos pueden ser utilizados para predecir la rugosidad superficial en el torneado en seco del acero AISI 316L. Sin embargo, como se puede observar en la misma tabla, los modelos obtenidos por redes neuronales artificiales producen mejores resultados comparados con los modelos por regresión múltiple. 


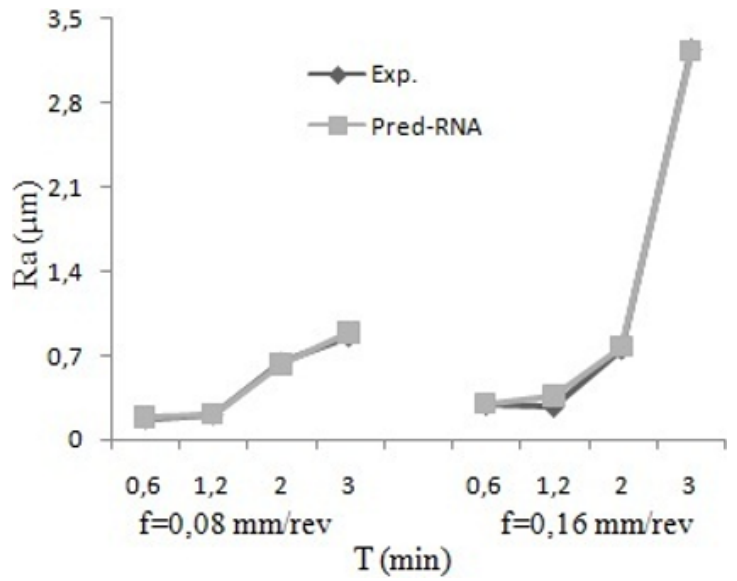

(a)

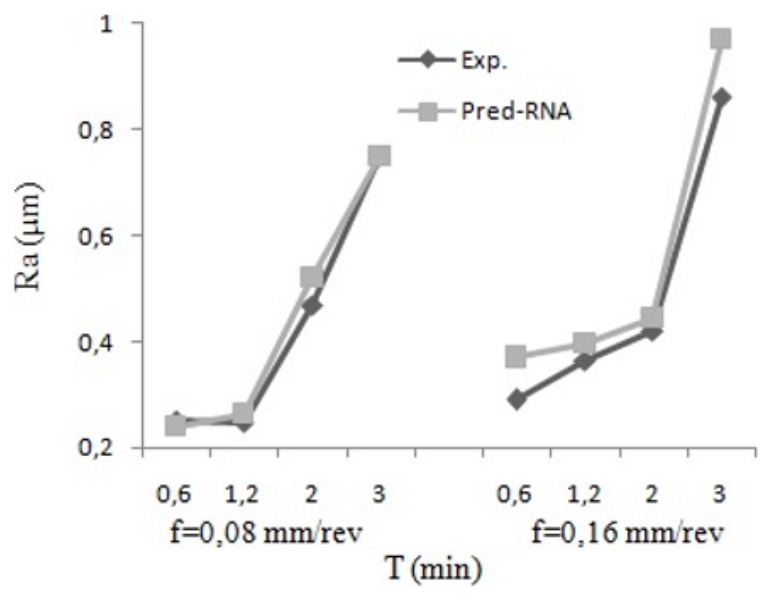

(b)

Figura 8. Valores medidos y estimados por redes neuronales artificiales para $\mathrm{v}=450 \mathrm{~m} / \mathrm{min}$, a) inserto $\mathrm{GC} 1115$ y b) inserto GC2015.

Tabla 4. Comparación de métodos propuestos

\begin{tabular}{cccc}
\hline Método & Ecuación & $\mathbf{E}_{\text {medio }}$ & $\mathbf{R 2}$ \\
\hline Regresión & -1 & 5,153 & 0,9 \\
múltiple & -2 & 5,552 & 0,8 \\
& -3 & 22,78 & 1 \\
Redes & $400 \mathrm{~m} / \mathrm{min}$ & 2,869 & 1 \\
neuronales & $450 \mathrm{~m} / \mathrm{min}$ & 6,946 & 1 \\
artificiales & & & \\
\hline
\end{tabular}

\section{Conclusiones}

En esta investigación se ha realizado un estudio para predecir la rugosidad superficial en el torneado en seco del acero AISI 316L. La influencia de variables como la velocidad, el avance y el tiempo de mecanizado fueron analizadas a través de un diseño factorial completo. Los modelos para predecir la rugosidad superficial fueron desarrollados a partir de los datos experimentales. De acuerdo con los resultados obtenidos en este trabajo se plantean las siguientes conclusiones:

- Los modelos desarrollados fueron evaluados por sus capacidades de predicción con los valores medidos experimentalmente.

- Los modelos propuestos pueden ser utilizados para predecir la rugosidad superficial en el torneado en seco del acero AISI 316L.

- El coeficiente de determinación mínimo alcanzado por los modelos fue de $80 \%$ y el máximo de $99 \%$, indicando la proporción de la variabilidad de los datos explicada por los modelos de regresión, en el caso del error medio absoluto el mínimo fue de 2,869\% y el máximo de 22,78.

- Los menores errores medios absolutos fueron obtenidos con los modelos implementados con redes neuronales artificiales.

- En investigaciones futuras se podrían desarrollar modelos basados en las redes neuronales y en la regresión múltiple que permitan un estudio de la economía del proceso de torneado en seco.

\section{Agradecimientos}

Los autores agradecen a la Secretaría de Educación Pública por proporcionar la beca de investigación en la Universidad Autónoma de Nuevo León (UANL) en México. También al Centro de Investigación e Innovación en Ingeniería Aeronáutica (CIIIA) por el apoyo financiero y tecnológico, además, por todas las facilidades brindadas para el desarrollo del trabajo de investigación.

\section{Referencias}

[1] D. Vasumathy, A. Meena, and M. Duraiselvam, "Experimental study on evaluating the effect of micro textured tools in turning aisi 316 austenitic stainless steel," Procedia Engineering, vol. 184, no. Supplement C, pp. 50-57, 2017, advances in Material \& Processing Technologies Conference. [Online]. Available: https://doi.org/10.1016/j.proeng.2017.04.070

[2] F. Mata-Cabrera, I. Hanafi, A. Khamlichi, A. Jabbouri, and M. Bezzazi, "Predicción de rugosidad en maquinado de compuestos con base de peek usando metodología de superficie de respuesta," Ingeniería, Investigación y Tecnología, vol. 14, no. 4, pp. 463-474, 2013. [Online]. Available: https://doi.org/10.1016/S1405-7743(13)72258-3 
[3] M. Mia and N. R. Dhar, "Prediction of surface roughness in hard turning under high pressure coolant using artificial neural network," Measurement, vol. 92, Supplement C, pp. 464-474, 2016. [Online]. Available: https: //doi.org/10.1016/j.measurement.2016.06.048

[4] A. Fernández-Abia, J. Barreiro, L. N. López de Lacalle, and S. Martínez, "Effect of very high cutting speeds on shearing, cutting forces and roughness in dry turning of austenitic stainless steels," The International Journal of Advanced Manufacturing Technology, vol. 57, no. 1-4, pp. 61-71, 2011. [Online]. Available: https://doi.org/10.1007/s00170-011-3267-9

[5] J. Zhou, V. Bushlya, and J. Stahl, "An investigation of surface damage in the high speed turning of inconel 718 with use of whisker reinforced ceramic tools," Journal of Materials Processing Technology, vol. 212, no. 2, pp. 372-384, 2012. [Online]. Available: https: //doi.org/10.1016/j.jmatprotec.2011.09.022

[6] T. Sata, Surface finish in metal cutting, 1964, vol. 12, pp. 190-197.

[7] G. R. Dickinson, "Paper 26: Survey of factors affecting surface finish," Proceedings of the Institution of Mechanical Engineers, Conference Proceedings, vol. 182, no. 11, pp. 135-147, 1967. [Online]. Available: https://doi.org/10. 1243/PIME_CONF_1967_182_311_02

[8] M. P. Groover, Fundamentos de manufactura moderna: materiales, procesos y sistemas, McGrawHill Interamericana de España S. L., 2007, no. 4. [Online]. Available: https://goo.gl/aJx7SQ

[9] I. Korkut, M. Kasap, I. Ciftci, and U. Seker, "Determination of optimum cutting parameters during machining of AISI 304 austenitic stainless steel," Materials \& Design, vol. 25, no. 4, pp. 303-305, 2004. [Online]. Available: https://doi.org/10.1016/j.matdes.2003.10.011

[10] Y. Morales Tamayo, Y. Zamora Hernández, P. Zambrano Robledo, R. Beltrán Reyna, and J. Pino Tarrago, "Investigação da influência dos parâmetros de corte na rugosidade superficial usando regressão múltipla," Revista Iberoamericana de Ingeniería Mecánica, vol. 20, no. 2, pp. 25-33, 2016. [Online]. Available: https://goo.gl/33SqTx

[11] U. Çaydaş and S. Ekici, "Support vector machines models for surface roughness prediction in cnc turning of aisi 304 austenitic stainless steel," Journal of Intelligent Manufacturing, vol. 23, pp. 639-650, 2012. [Online]. Available: https://doi.org/10.1007/s10845-010-0415-2
[12] C. Ahilan, S. Kumanan, N. Sivakumaran, and J. E. R. Dhas, "Modeling and prediction of machining quality in cnc turning process using intelligent hybrid decision making tools," Applied Soft Computing, vol. 13, no. 3, pp. 1543-1551, 2013, hybrid evolutionary systems for manufacturing processes. [Online]. Available: https://doi.org/10.1016/j.asoc.2012.03.071

[13] D. P. Selvaraj, P. Chandramohan, and M. Mohanraj, "Optimization of surface roughness, cutting force and tool wear of nitrogen alloyed duplex stainless steel in a dry turning process using taguchi method," Measurement, vol. 49, Supplement C, pp. 205-215, 2014. [Online]. Available: https: //doi.org/10.1016/j.measurement.2013.11.037

[14] W. S. Lin, "The study of high speed fine turning of austenitic stainless steel," Journal of Achievements in Materials and Manufacturing Engineering, vol. 27, no. 2, pp. 191-194, 2008. [Online]. Available: https://goo.gl/UWA5LL

[15] A. I. Hernández-Abia, J. Barreiro, L. N. López de Lacalle, and S. Martínez Pellitero, "Behavior of austenitic stainless steels at high speed turning using specific force coefficients," The International Journal of Advanced Manufacturing Technology, vol. 62, no. 5-8, pp. 505-515, 2012. [Online]. Available: https://doi.org/10.1007/s00170-011-3846-9

[16] C. M. ao and J. P. Davim, "Finite element modelling of machining of aisi 316 steel: $\mathrm{Nu}-$ merical simulation and experimental validation," Simulation Modelling Practice and Theory, vol. 18, no. 2, pp. 139-56, 2010. [Online]. Available: https://doi.org/10.1016/j.simpat.2009.10.001

[17] N. I. Galanis and D. E. Manolakos, "Surface roughness prediction in turning of femoral head," The International Journal of Advanced Manufacturing Technology, vol. 51, no. 1-4, pp. 79-86, 2010. [Online]. Available: https://doi.org/10.1007/s00170-010-2616-4

[18] K. S. Sangwan, S. Saxena, and G. Kant, "Optimization of machining parameters to minimize surface roughness using integrated ann-ga approach," Procedia CIRP, vol. 29, Supplement C, pp. 305-310, 2015, the 22nd CIRP Conference on Life Cycle Engineering. [Online]. Available: https://doi.org/10.1016/j.procir.2015.02.002

[19] E. Kilickap, M. Huseyinoglu, and A. Yardimeden, "Optimization of drilling parameters on surface roughness in drilling of aisi 1045 using response surface methodology and genetic algorithm," The International Journal of 
Advanced Manufacturing Technology, vol. 52, no. 1-4, pp. 79-88, 2011. [Online]. Available: https://doi.org/10.1007/s00170-010-2710-7

[20] R. E. Harber, J. E. Jiménez, A. Jiménez, and J. López-Coronado, "Modelo matemático para la predicción del esfuerzo de corte en el mecanizado a alta velocidad," Revista de Metalurgia, vol. 40, no. 4, pp. 247-258, 2004. [Online]. Available: http: //dx.doi.org/10.3989/revmetalm.2004.v40.i4.272

[21] I. Asiltũrk and M. Çunkaş, "Modeling and prediction of surface roughness in turning operations using artificial neural network and multiple regression method," Expert Systems with Applications, vol. 38 , no. 5, pp. 5826-5832, 2011. [Online]. Available: https://doi.org/10.1016/j.eswa.2010.11.041

[22] K. V. B. S. Kalyan Kumar and S. K. Choudhury, "Investigation of tool wear and cutting force in cryogenic machining using design of experiments," Journal of Materials Processing Technology, vol. 203, no. 1, pp. 95-101, 2008. [Online]. Available: https: //doi.org/10.1016/j.jmatprotec.2007.10.036

[23] D. Montgomery, Design and Analysis of Experiments (fifth edition). John Wiley \& Sons, Ltd., 2002. [Online]. Available: http://dx.doi.org/10.1002/qre.458
[24] J. Montaño Moreno, "Redes neuronales artificiales aplicadas al análisis de datos," Ph.D. dissertation, Universitat de Les Illes Balears. Islas Baleares, España, 2002. [Online]. Available: https://goo.gl/yVkDrN

[25] V. N. Gaitonde, S. R. Karnik, B. Siddeswarappa, and B. T. Achyuta, "Integrating box-behnken design with genetic algorithm to determine the optimal parametric combination for minimizing burr size in drilling of aisi 3161 stainless steel," The International Journal of Advanced Manufacturing Technology, vol. 37, no. 3-4, pp. 230-240, 2008. [Online]. Available: https://doi.org/10.1007/s00170-007-0957-4

[26] Z. Zhimin, Z. Yuanliang, L. Xiaoyan, Z. Huiyuan, and S. Baoyuan, "Influences of various cutting parameters on the surface roughness during turnings stainless steel," Acoustical Physics, vol. 57, no. 1, pp. 114-120, 2011. [Online]. Available: https://doi.org/10.1134/S1063771011010209

[27] J. Campos Rubio, T. H. Panzera, A. M. Abrao, P. F. Faria, and J. Paulo Davim, "Effects of high speed in the drilling of glass whiskerreinforced polyamide composites (pa66 gf30): statistical analysis of the roughness parameters," Journal of Composite Materials, vol. 45, no. 13, pp. 1395-1402, 2011. [Online]. Available: https://doi.org/10.1177/0021998310381540 


\title{
OBTENCIÓN DE ACS CON ENERGÍA SOLAR EN EL CANTÓN CUENCA Y ANÁLISIS DE LA CONTAMINACIÓN AMBIENTAL OBTAINING OF SHW WITH SOLAR ENERGY IN THE CANTON CUENCA AND ANALYSIS OF ENVIRONMENTAL POLLUTION
}

\author{
John Calle Sigüencia ${ }^{1, *}$, Óscar Tinoco Gómez ${ }^{1}$
}

\section{Resumen}

En este documento se encuentra el análisis para determinar la factibilidad de implementación de calentadores solares para obtener agua caliente sanitaria (ACS) en el Ecuador, en la provincia del Azuay, en el cantón Cuenca, con el fin de disminuir la contaminación ambiental provocada por el uso de combustibles fósiles. El proyecto considera la puesta en marcha de una red meteorológica y toma de datos de radiación solar global en 16 puntos ubicados zonas pobladas del cantón durante los años 2014 y 2015, posteriormente a través de un trabajo de campo se realiza un diagnóstico para establecer cuáles son los actuales sistemas usados para obtener ACS, también se analizan de manera teórica y práctica las eficiencias de dos tipos de calentadores solares de tubos de vacío para producción de ACS; con los datos de radiación medidos se modela aplicando las ecuaciones de transferencia de calor y se establece la factibilidad de implementación en función de la energía solar medida; finalmente, se realiza una comparación para determinar cuál sería la disminución de emanaciones de $\mathrm{CO}_{2}$ si se ejecutaría esta propuesta. Los resultados obtenidos indican que el $82 \%$ de familias utiliza ACS, de estas el $65 \%$ emplean sistemas a base de GLP y, que el $44 \%$ de la demanda de energía para obtener ACS puede ser cubierta con energía solar necesitando utilizar sistemas auxiliares para garantizar un abastecimiento constante. La implementación de estos sistemas permitiría reducir 108537 tn $\mathrm{CO}_{2}$ eq al año.

Palabras clave: calentador solar, contaminación ambiental, energía solar, tubos de vacío

\section{Abstract}

This document contains the analysis to determine the feasibility of implementing solar water heaters to obtain Sanitary hot water(SHW) in Ecuador, in the province of Azuay, in the Canton of Cuenca, in order to reduce the environmental pollution caused by the use of fossil fuels. The project considers the implementation of a meteorological network and data collection of global solar radiation in 16 points located in populated areas of the Canton during the years 2014 and 2015. Then, through a field work a diagnosis is made to establish which are the systems which are currently used to obtain SHW, two solar vacuum tube heaters for SHW production are also located and characterized with their corresponding equations. With the measured radiation data, we stablish the model applying the equations and establishing the feasibility of implementation based on the measured solar energy. Finally, a comparison is made to determine what would be the decrease of $\mathrm{CO}_{2}$ emissions if the implementation would be carried out. The obtained results indicate that in $82 \%$ of households it uses SHW and of these $65 \%$ use LPG-based systems, which $44 \%$ of the energy demand to obtain SHW can be covered with solar energy. Therefore auxiliary systems can be used to guarantee a constant supply and that with the implementation it would be possible to reduce $108537 \mathrm{t} \mathrm{CO}_{2}$ eq per year.

Keywords: Solar heater, environmental pollution, solar energy, vacuum tubes

\footnotetext{
$\overline{1, *}$ Unidad de posgrados de la Facultad de Ingeniería Industrial, Universidad Nacional Mayor de San Marcos, Lima - Perú. Autor para correspondencia : jics_1970@hotmail.com, (D) http://orcid.org/0000-0002-2148-3297

(D) https://orcid.org/0000-0002-2548-2160
}

Recibido: 21-12-2017, aprobado tras revisión: 27-12-2017

Forma sugerida de citación: Calle, J.; Tinoco, O. (2018). «Obtención de ACS con energía solar en el cantón Cuenca y análisis de la contaminación ambiental». INGENIUS. N. ${ }^{\circ} 19$, (enero-junio). pp. 89-101. DOI: https://doi.org/10.17163/ ings.n19.2018.09. 


\section{Introducción}

Desde la década de los 70 la preocupación por el medioambiente ha sido especialmente intensa, generando una serie de actuaciones, conferencias y acuerdos internacionales [1], entre sus puntos fundamentales está la disminución de la producción de $\mathrm{CO}_{2}$ y los gases de efecto invernadero, pero los resultados de las mismas han caído en buenas intenciones y no se han conseguido acciones eficientes que favorezcan este propósito a gran escala.

El uso de energías renovables ha generado un incremento en el desarrollo tecnológico siendo cada vez más fiables y con un alto rendimiento, lo que ha llevado a que su producción, comercialización e implementación aumente constantemente tanto para uso doméstico como para la industria.

Por los años 70 se empezó a utilizar la energía solar como una de las principales opciones para el uso en beneficio de las personas, países como Estados Unidos, Francia, Alemania, España, prestan gran atención al uso de la energía solar con fines térmicos y fotovoltaicos e introducen importantes porcentajes de generación energética. Para el año 2016 el $94 \%$ de los sistemas térmicos solares instalados en el mundo se utilizan para obtener agua caliente [2].

El Ecuador es un país privilegiado en lo que al recurso solar se refiere, por su ubicación geográfica ya que el ángulo de incidencia de la radiación solar, es casi perpendicular a la superficie durante todo el año, situación que no ocurre en otros sitios del planeta, en donde el ángulo varía acorde a las estaciones del año. Esta ventaja posicional se traduce en la recepción de una mayor y constante cantidad de radiación solar (Figura 1), misma que varía dentro del territorio nacional únicamente por condiciones geográficas locales. La condición antes manifestada no ha sido considerada para aprovecharla en sistemas que favorezcan la disminución de emisiones de $\mathrm{CO}_{2}$ con especial atención en lo que respecta a la producción de ACS considerando una dotación constante que mantenga las condiciones de confort que se obtiene con otras fuentes.

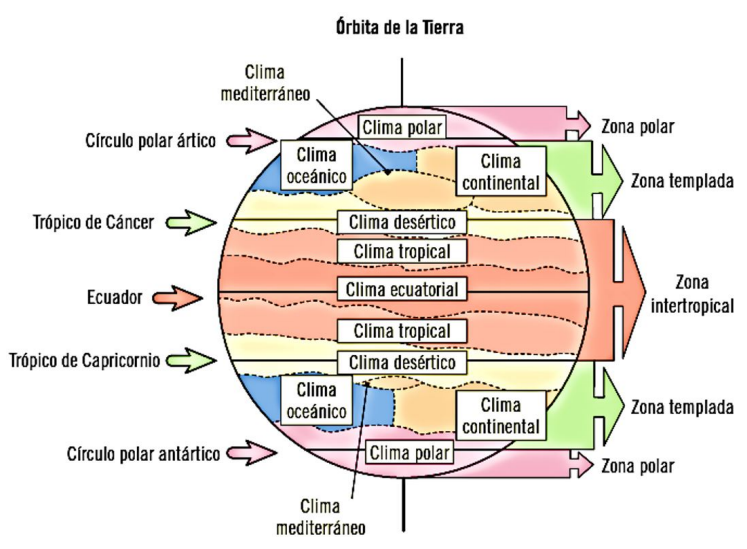

Figura 1. Zonas térmicas de la Tierra [3]

\section{Materiales y métodos}

La investigación desarrollada es de tipo cuasi experimental; ya que no hay un control efectivo de las variables de selección y para su desarrollo se integran un conjunto de actividades metódicas y técnicas que se realizan para recabar la información y datos necesarios sobre el tema a investigar y el problema a resolver [4].

Para el proyecto presentado se requiere un cálculo del porcentaje de $\mathrm{CO}_{2}$ por kilogramo de combustible quemado en un período definido sin sistemas solares térmicos (grupo de control) y el cálculo del porcentaje de $\mathrm{CO}_{2}$ con aplicación de sistemas solares térmicos (grupo experimental). Para la investigación se han considerado 4 fases como se muestra en la Figura 2.

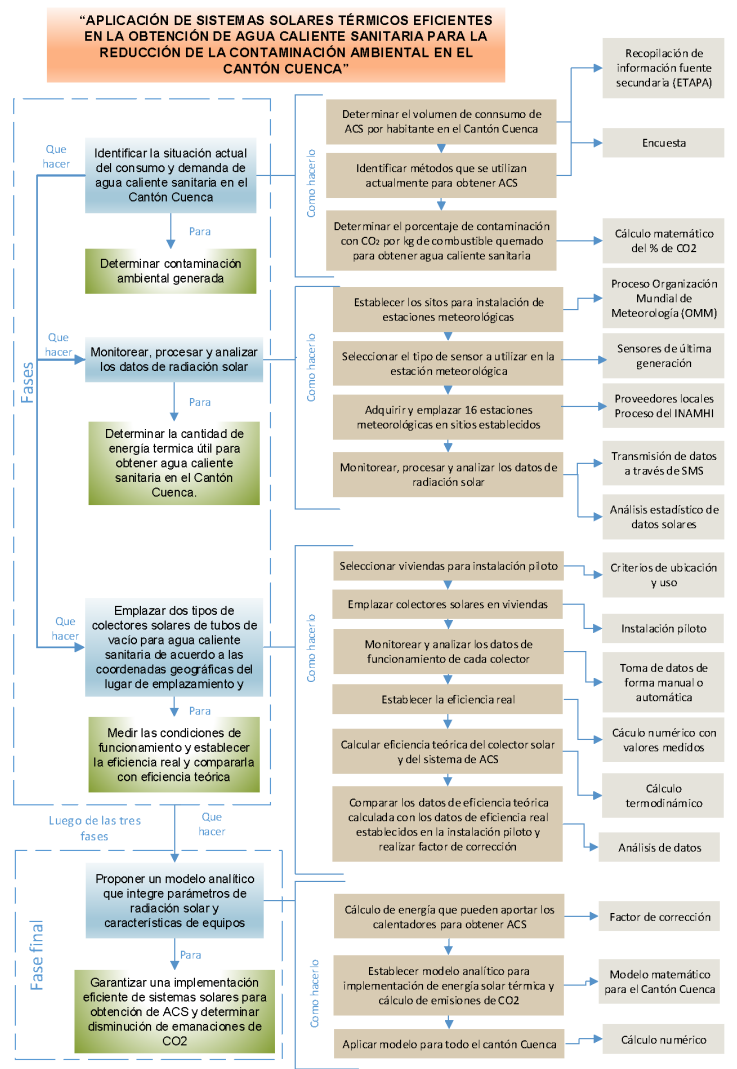

Figura 2. Esquema del diseño de la investigación

\subsection{Análisis de la situación actual de consumo y demanda de ACS en el cantón Cuenca y determinación de porcentaje de $\mathrm{CO}_{2}$ emi- tido al ambiente}

En este punto se determina el consumo de agua por habitante a través de datos de fuente secundaria que en este caso se recopilan de la Empresa Pública Municipal de Telecomunicaciones, Agua Potable, Alcantarillado y Saneamiento Ambiental (ETAPA) y se completa el análisis con una encuesta en donde se identifica el consumo y demanda de ACS, además, con la misma se 
identifican los métodos que se utilizan para producir agua caliente: calentadores a gas, calentadores eléctricos, calentadores solares y otros. Finalmente, mediante un cálculo matemático se determinará el porcentaje de contaminación con $\mathrm{CO}_{2}$ por kilogramo de combustible quemado para obtener ACS.

Para la aplicación de la encuesta se realiza la proyección de la población para el año 2016 empleando el método parabólico [5], basándose en la información de los censos de los años 1990, 2001 y 2010 [6] como se muestra en la Tabla 1.

Tabla 1. Proyección de número de habitantes del cantón Cuenca para el año 2016

\begin{tabular}{cccc}
\hline Año & Urbana & Rural & Total \\
\hline 1990 & 194981 hab. & 136047 hab. & 331028 hab. \\
2001 & 277374 hab. & 140258 hab. & 417632 hab. \\
2010 & 331888 hab. & 173697 hab. & 505585 hab. \\
$\mathbf{2 0 1 6}$ & $\mathbf{3 6 1} \mathbf{7 8 1}$ hab. & $\mathbf{2 1 0} \mathbf{9 8 6}$ hab. & $\mathbf{5 7 2} \mathbf{7 6 7}$ hab. \\
\hline
\end{tabular}

La población en estudio está conformada por el número de hogares en el cantón Cuenca. Se estima que un hogar promedio está integrado por 5 miembros, lo que resulta en un total de 114553 hogares para todo el cantón divididos en 72356 para la zona urbana y 42197 en la zona rural.

Para determinar la muestra se aplica la ecuación (1) considerando el nivel de confianza $\mathrm{k}=95 \%$, límite de error $\mathrm{e}=0,06$, probabilidad de éxito $\mathrm{p}=0,6 \mathrm{y}$ probabilidad de fracaso $\mathrm{q}=0,4$.

$$
n=\frac{k^{2} \times p \times q \times N}{\left(e^{2} \times(N-1)\right)+k^{2} \times p \times q}
$$

La muestra significativa con la que se va a trabajar es de 531 hogares divididos en 266 en la zona urbana y 265 en la zona rural; en esta última se realiza una estratificación en función del número de pobladores de cada parroquia debido a las particularidades geográficas que presentan cada una; en la zona urbana no se estratifica pues las condiciones de todas las parroquias son similares.

Para el levantamiento de la información como instrumento se utiliza un cuestionario. Considerando el tránsito de la variable a sus dimensiones o componentes, luego a los indicadores y, finalmente, a los ítems o reactivos [4], el análisis establecido se muestra en la Tabla 2.

Tabla 2. Análisis de tránsito de variables para cuestionario

\begin{tabular}{|c|c|c|c|c|c|c|c|}
\hline \multicolumn{8}{|c|}{ Análisis sobre uso de ACS en el cantón Cuenca } \\
\hline Variable & Dimensión & Indicadores & \multicolumn{5}{|c|}{ Ítems } \\
\hline Uso de ACS & $\begin{array}{l}\text { Actividades de } \\
\text { limpieza } \\
\text { cotidiana }\end{array}$ & Si utiliza o no utiliza & \multicolumn{5}{|c|}{$\begin{array}{l}\text { Utiliza agua caliente para sus actividades regulares como bañarse, lavado de manos, } \\
\text { lavado de vajilla, lavado de ropa, etc. (Marque con una "X" su respuesta). } \\
\text { SÍ ...... NO....... }\end{array}$} \\
\hline No uso de ACS & $\begin{array}{l}\text { Factor de } \\
\text { consideración }\end{array}$ & $\begin{array}{l}\text { Jerarquía de preferencia } \\
\text { del por qué no utiliza ACS } \\
\text { considerando costo, } \\
\text { contaminación y facilidad } \\
\text { de comprar e instalar. }\end{array}$ & \multicolumn{5}{|c|}{$\begin{array}{l}\text { Si su respuesta es NO, escriba en el casillero que está en blanco los números del } 1 \text { al } \\
4 \text {, considerando el } 1 \text { al más importante y } 4 \text { al menos importante. } \\
\text { Muy costoso........... } \\
\text { Contamina el ambiente...... } \\
\text { Difícil de comprar...... } \\
\text { Difícil de instalar......... }\end{array}$} \\
\hline $\begin{array}{c}\text { Número de } \\
\text { habitantes por hogar }\end{array}$ & Contable & $\begin{array}{l}\text { Número de habitantes por } \\
\text { hogar }\end{array}$ & \multicolumn{5}{|c|}{$\begin{array}{l}\text { Incluido usted, } ¿ \text { cuántas personas habitan en su casa? } \\
\ldots \ldots \ldots \ldots \ldots \ldots \text { personas } \\
\end{array}$} \\
\hline \multirow{9}{*}{$\begin{array}{l}\text { Tipo de sistema que } \\
\text { utiliza para obtener } \\
\text { ACS en la vivienda }\end{array}$} & \multirow{9}{*}{$\begin{array}{l}\text { Identificación } \\
\text { del tipo de } \\
\text { sistema para } \\
\text { ACS }\end{array}$} & \multirow{9}{*}{$\begin{array}{c}\text { Determinación de sistema } \\
\text { actual para obtener ACS } \\
\text { considerando: Gas (GLP); } \\
\text { resistencia eléctrica, } \\
\text { inducción eléctrica, } \\
\text { calentador solar, solar y } \\
\text { gas, solar y resistencia } \\
\text { eléctrica, otro en } \\
\text { actividades regulares: } \\
\text { ducha, lavamanos, } \\
\text { fregadero de cocina y } \\
\text { lavandería }\end{array}$} & \multicolumn{5}{|c|}{$\begin{array}{l}\text { Indique el tipo de sistema de calentamiento de agua que utiliza en su hogar para las } \\
\text { siguientes actividades. (Marque con una "X" solamente en los que utiliza agua } \\
\text { caliente) }\end{array}$} \\
\hline & & & \begin{tabular}{|c|} 
Actividad \\
Sistema \\
\end{tabular} & Ducha & Lavamanos & \begin{tabular}{|c|}
$\begin{array}{c}\text { Fregadero de } \\
\text { cocina }\end{array}$ \\
\end{tabular} & Lavandería \\
\hline & & & A gas (GLP) & & & & \\
\hline & & & Resistencia eléctrica & & & & \\
\hline & & & Inducción eléctrica & & & & \\
\hline & & & & & & & \\
\hline & & & Solar y Gas (GLP) & & & & \\
\hline & & & $\begin{array}{c}\text { Solar-Resistencia } \\
\text { eléctrica }\end{array}$ & & & & \\
\hline & & & Otro & & & & \\
\hline \multirow{5}{*}{$\begin{array}{l}\text { Tiempo utilizado } \\
\text { para actividades de } \\
\text { limpieza con ACS }\end{array}$} & \multirow{5}{*}{$\begin{array}{l}\text { Determinación } \\
\text { de tiempo }\end{array}$} & \multirow{5}{*}{$\begin{array}{l}\text { Rango de tiempo por } \\
\text { actividades regulares: } \\
\text { ducha, lavamanos, } \\
\text { fregadero de cocina y } \\
\quad \text { lavandería }\end{array}$} & \multicolumn{5}{|c|}{$\begin{array}{l}\text { Considerando lo marcado en la tabla anterior determine el tiempo total diario para } \\
\text { cada actividad sumando el tiempo ocupado por cada miembro de familia. (Marque } \\
\text { con una «X»su respuesta). }\end{array}$} \\
\hline & & & Tiempo Actividad & De 30 a 45 min & De 45 a $60 \mathrm{~min}$ & De $60 \mathrm{~min}$ a $75 \mathrm{~min}$ & Mayor a $75 \mathrm{~min}$ \\
\hline & & & Ducha & & & & \\
\hline & & & Lavamanos & & & & \\
\hline & & & $\begin{array}{c}\text { Fregadero de cocina } \\
\text { Lavandería } \\
\end{array}$ & & & & \\
\hline $\begin{array}{l}\text { Calidad del sistema } \\
\text { de ACS utilizado }\end{array}$ & $\begin{array}{l}\text { Atribución del } \\
\text { desempeño }\end{array}$ & $\begin{array}{l}\text { Grado de percepción de la } \\
\text { calidad del sistema de } \\
\text { ACS utilizado }\end{array}$ & \multicolumn{5}{|c|}{$\begin{array}{c}\text { ¿Cómo califica el actual sistema de calentamiento de agua que Ud. dispone? (Marque } \\
\text { con una «X» la respuesta correspondiente, la misma que es única). } \\
\text { Muy bueno...... Bueno...... Regular..... Malo..... }\end{array}$} \\
\hline $\begin{array}{l}\text { Factores para elegir } \\
\text { sistema }\end{array}$ & $\begin{array}{l}\text { Atribución de } \\
\text { implementación }\end{array}$ & $\begin{array}{l}\text { Grado de percepción y } \\
\text { jerarquización de factores } \\
\text { para elegir sistema de } \\
\text { ACS para la vivienda }\end{array}$ & \multicolumn{5}{|c|}{$\begin{array}{l}\text { ¿Cuáles son los factores que consideró al momento de elegir su actual sistema de } \\
\text { calentamiento de agua? (Escriba en el casillero que está en blanco los números del } 1 \text { al } \\
4 \text {, considerando el } 1 \text { al más importante y } 4 \text { al menos importante). } \\
\text { Bajo costo...... Fácil de instalar...... } \\
\text { No contamina...... Fácil de comprar...... }\end{array}$} \\
\hline
\end{tabular}


Para la recolección de datos se aplica el cuestionario de acuerdo con el análisis realizado en los párrafos anteriores; un grupo de encuestadores visitaron los hogares y solicitaron se complete el mismo.

La cantidad de energía que se requiere para cubrir la demanda, considerando los valores promedio de consumo de ACS en ducha, lavamanos y en el fregadero de cocina determinados en la encuesta, se obtiene de la ecuación (2):

$$
D_{A C S}=V_{A C S} \times \rho_{a} \times C_{p} \times\left(T_{u s o}-T_{r e d}\right)
$$

Donde:

$\mathrm{D}_{\mathrm{ACS}}=$ Demanda de ACS $(\mathrm{J})$

$\mathrm{V}_{\mathrm{ACS}}=$ Volumen de consumo de $\operatorname{ACS}\left(\mathrm{m}^{3} / \mathrm{mes}\right)$

$\rho_{\mathrm{a}}=$ Densidad del agua $1000 \mathrm{~kg} / \mathrm{m}^{3}$

$\mathrm{C}_{\mathrm{p}}=$ Calor específico del agua $\left(4187 \mathrm{~J} /\left(\mathrm{kg} \cdot{ }^{\circ} \mathrm{C}\right)\right)$

$\mathrm{T}_{\mathrm{uso}}=$ Temperatura de consumo $\left({ }^{\circ} \mathrm{C}\right)$

$\mathrm{T}_{\text {red }}=$ Temperatura de la red $\left({ }^{\circ} \mathrm{C}\right)$

Para calcular las emisiones de $\mathrm{CO}_{2}$ generadas al momento de obtener ACS, se multiplica la energía requerida para la obtención de ACS por un factor de emisiones que para GLP es de 0,234 y para electricidad $0,385 \mathrm{~kg}$ de $\mathrm{CO}_{2} \mathrm{eq} / \mathrm{kWh}[7]$.

\subsection{Monitoreo, procesamiento y análisis de los datos de radiación solar para el cantón Cuenca}

Para esta segunda fase del proyecto se trabaja en la selección y emplazamiento de las estaciones meteorológicas de acuerdo con las recomendaciones realizadas por la Organización Mundial de Meteorología (OMM). Se realiza un control de calidad de datos y los ajustes correspondientes para finalmente cuantificar la energía existente en cada uno de los sectores en donde se encuentran emplazadas las estaciones meteorológicas y que están asociados a las parroquias urbanas y rurales del cantón Cuenca.

\subsubsection{Emplazamiento de estaciones meteoroló- gicas en el cantón Cuenca}

En el año 2013 la Universidad Politécnica Salesiana, UPS, y el Instituto Nacional de Eficiencia Energética y Energías Renovables, INER, instalaron una red de 16 estaciones meteorológicas repartidas en puntos estratégicos del cantón Cuenca con el propósito de medir variables meteorológicas y estos datos poderlos utilizar en proyectos para implantación de sistemas energéticos fundamentados en energías renovables [8].

Para la correcta definición geográfica de los sitios de emplazamiento de las estaciones se trabajaron en seis etapas las cuales se listan a continuación [9].
- Determinación de datos de posibles lugares para emplazamiento.

- Visitas de campo.

- Selección de criterios.

- Análisis espacial.

- Valoración y elección de lugares de emplazamiento.

- Selección y emplazamiento de estaciones meteorológicas

\subsubsection{Caracterización de la radiación solar en el cantón Cuenca durante los años 2014- 2015}

Para este proceso se recopila la información de radiación global medida por la estación meteorológica y se realiza un control de calidad de los datos obtenidos, ya que, por condiciones de los sitios de emplazamiento o por circunstancias no controladas de los equipos pueden existir datos faltantes en fechas establecidas; a continuación, se procede a realizar la complementación correspondiente, se cuantifica la radiación existente y se calcula el aporte energético que la radiación ofrece en las diferentes zonas para cada mes en los años 2014 y 2015 .

La información faltante se completa utilizando el modelo matemático de Angström - Prescott modificada por Page, el cual permite estimar el recurso solar en una zona determinada [10]. El procedimiento consiste en hacer uso de varias ecuaciones correlacionadas entre sí, las cuales permiten calcular la radiación extraterrestre (He) según la ubicación geográfica del área de interés, para luego emplear la ecuación de Page y así obtener la radiación sobre una superficie horizontal (Ho). La correlación de Angström - Page para determinar la radiación solar global faltante sobre una superficie horizontal se muestra en la Tabla 3.

Tabla 3. Correlación de Angström - Page para determinar la radiación solar global [10]

\begin{tabular}{|c|c|}
\hline Descripción & Ecuación \\
\hline $\begin{array}{c}\text { Horas } \\
\text { teóricas de } \\
\text { brillo solar }(\mathrm{h})\end{array}$ & $N=\frac{2}{15} \cos ^{-1}(-\tan \phi \cdot \tan \delta)$ \\
\hline $\begin{array}{c}\text { Radiación } \\
\text { extraterrestre } \\
\left(\mathrm{J} / \mathrm{m}^{2}\right) \\
\end{array}$ & $\begin{array}{l}H_{e}=\frac{24}{\pi} I_{s c}\left[1+0.033 \cos \frac{360 \times z}{365}\right] \cdot[\cos \rho \\
\left.\quad \cdot \cos \delta \cdot \sin h_{s}+\frac{2 \pi h_{s}}{360} \sin \phi \cdot \sin \rho\right]\end{array}$ \\
\hline Horas reales $(\mathrm{h})$ & $H S P_{\text {Ecuador }}=9$ \\
\hline $\begin{array}{l}\text { Radiación solar } \\
\text { en superficie } \\
\text { horizontal } \\
\left(\mathrm{Wh} / \mathrm{m}^{2}\right)\end{array}$ & $H_{0}=H_{e}\left(a+b \frac{n_{\text {reales }}}{N}\right)$ \\
\hline
\end{tabular}


Para realizar los cálculos correspondientes, se toman los valores complementados diarios y las horas de sol establecidas, se suman los valores para encontrar el total diario y se suman los totales diarios de cada mes para obtener el total mensual, por último, se toman los totales mensuales y se obtiene el valor de energía incidente total anual.

\subsection{Caracterización de colectores solares de tubos de vacío y tubo de vacío con tubos de calor}

Para el caso en estudio se utilizan dos tipos de colectores solares que se instalan en dos viviendas del cantón Cuenca; el primero es un calentador de tubos de vacío y el otro es un calentador de tubos de vacío con tubo de calor, que son los que comercialmente se pueden conseguir en el Ecuador; para cada uno se realiza el análisis teórico correspondiente aplicando los principios de la termodinámica y la transferencia de calor. Con los colectores instalados se determinan las eficiencias reales y se compararan con los datos teóricos calculados, se establece la corrección correspondiente y se obtiene el modelo matemático específico para cada uno.

El calor útil $(\mathrm{Qu})$ que vendría a ser igual al calor incidente (Qinc) que es el que proviene de la radiación solar menos el calor de pérdidas (Qper) que se dan en el proceso de transferencia de calor se calcula con la ecuación (3).

$$
Q_{u}=Q_{i n c}-Q_{p e r}
$$

Para determinar la eficiencia $\eta$ de los colectores solares se utiliza la ecuación (4):

$$
\eta=\frac{Q_{u}}{Q_{i n c}}
$$

\subsubsection{Caracterización de calentador solar de tubo de vacío.}

Para el cálculo del calor incidente en vatios se tiene la ecuación (5)

$$
Q_{i n c}=I_{p} \times \alpha_{s} \times A
$$

Donde:

$\mathrm{I}_{\mathrm{p}}$ es la irradiación promedio en la ciudad de Cuenca. A es el área de captación de la radiación multiplicado por el número de tubos.

$\alpha_{S}$ es el factor de corrección de la radiación incidente que llega a los tubos de vacío y es determinado por la Ecuación (6):

$$
\alpha_{s}=\frac{\tau \alpha}{1-(a-\alpha) \rho_{d}}
$$

Donde:

$\tau$ es la transmisividad de los tubos de vidrio. $\alpha$ es la absortividad de los tubos.

$\rho_{d}$ es la reflectancia difusa de los tubos.

Para el cálculo del área de incidencia $\left(\mathrm{m}^{2}\right)$ se debe tomar en cuenta que la radiación solar cualquiera que sea la ubicación del sol solamente afectará la mitad de los tubos de vacío, por lo tanto, solo se tomará en cuenta la mitad de la periferia como se muestra en la ecuación (7):

$$
A=\frac{d_{i} \times \pi \times \text { número de tubos }}{2}
$$

El siguiente proceso es determinar las pérdidas por conducción, convección y radiación en todo el calentador como se muestra en la Figura 3.

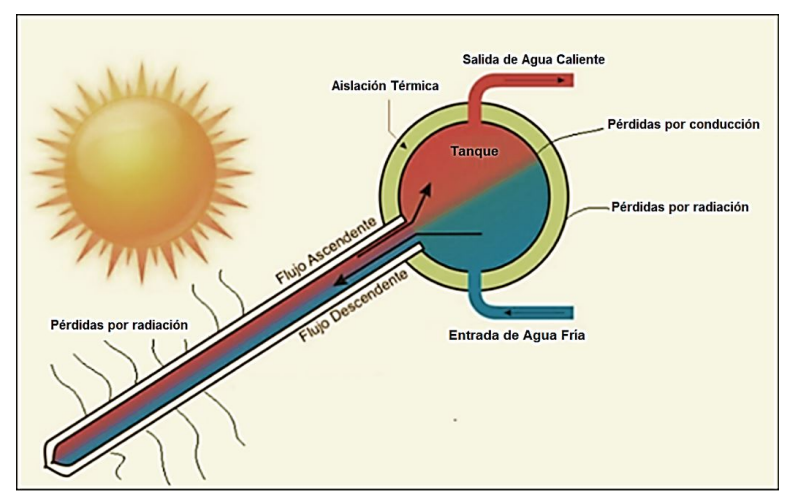

Figura 3. Pérdidas de calor en un calentador solar de tubos de vacío [11].

El total de las pérdidas $\left(\mathrm{TQ}_{\mathrm{per}}\right)(\mathrm{W})$ está dado por la sumatoria de las pérdidas obtenidas en los tubos de vacío $\left(\mathrm{Q}_{\mathrm{p}-\text { Tubos }}\right)$ y las pérdidas del tanque acumulador $\left(\mathrm{Q}_{\mathrm{p} \text {-Tanque }}\right)$, por lo tanto, la expresión para el cálculo de las pérdidas totales está dado por la ecuación (8):

$$
Q_{\text {per }}=Q_{p-\text { Tubos }}+Q_{p-\text { Tanque }}
$$

En el caso de los tubos de vacío las pérdidas se generan únicamente por radiación [12], ya que esta es su ventaja frente a otros sistemas; para el cálculo de las pérdidas en los tubos se tiene la ecuación (9):

$$
Q_{p-T u b o s}=U_{l r} \times A\left(T_{c}-T_{a}\right)
$$

Donde:

$\mathrm{U}_{\mathrm{lr}}$ es el coeficiente de pérdidas de calor por radiación de los tubos.

A es el área de captación.

$\mathrm{T}_{\mathrm{c}}$ es la temperatura de la cubierta.

$\mathrm{T}_{\mathrm{a}}$ es la temperatura ambiente. 
El cálculo de $\mathrm{U}_{\mathrm{lr}}$ se lo realizará mediante la fórmula propuesta por Duffie \& Beckman [13] en la cuál relacionan las pérdidas por radiación de la superficie del tubo receptor hacia el tubo de cubierta $\left(\mathrm{h}_{r,}, r-c\right)$ y las pérdidas por radiación del tubo de cubierta hacia el ambiente $\left(\mathrm{h}_{r,}, c-a\right)$ como se muestra en la Ecuación 10.

$$
U_{i r}=\left[\frac{A_{r}}{h_{w}+h_{r, \quad c-a} \times A_{c}}+\frac{1}{h_{r, \quad r-c}}\right]^{-1}
$$

Donde:

$A_{\mathrm{r}}$ representa el área del tubo receptor.

$A_{\mathrm{c}}$ es el área de la cubierta o tubo exterior.

$\mathrm{h}_{\mathrm{w}}$ es el coeficiente de convección en función del viento.

Para el cálculo del coeficiente de convección $\left(\frac{\mathrm{w}}{\mathrm{m}^{2} \times{ }^{\circ} \mathrm{C}}\right)$ se utilizará la ecuación (11):

$$
h_{w}=N_{u} \times \frac{K}{D}
$$

Donde:

$\mathrm{N}_{\mathrm{u}}$ es el número de Nusselt.

$\mathrm{K}$ es el coeficiente de conductividad térmica.

$\mathrm{D}$ es el diámetro del tubo.

El coeficiente de transferencia de calor entre los dos tubos concéntricos, el tubo receptor y el tubo de la cubierta $h_{r}, r-c\left(\frac{w}{m^{2} \times{ }^{\circ} \mathrm{C}}\right)$ no se ve atenuado por el vacío existente entre estos, por lo tanto, su valor será calculado mediante la ecuación (12):

$$
h_{r, \quad r-c}=\frac{\sigma\left(T_{r}^{2}+T_{c}^{2}\right) \times\left(T_{r}+T_{c}\right)}{\frac{1-\varepsilon_{1}}{\varepsilon_{1}}+\frac{1}{F_{12}}+\frac{1-\varepsilon_{2} \times A_{r}}{\varepsilon_{2} \times A_{c}}}
$$

Donde:

$\varepsilon_{1}$ es la emisividad del tubo receptor.

$\varepsilon_{2}$ es la emisividad del tubo de cubierta.

$\mathrm{F}_{12}$ es el factor de visión.

$\mathrm{T}_{\mathrm{c}}$ es la temperatura de la cubierta.

$\mathrm{T}_{\mathrm{r}}$ es la temperatura del receptor.

Para el cálculo de pérdidas en el tanque acumulador se realiza una analogía con redes de resistencias térmicas ya que el tanque está conformado por tres materiales como se muestra en la Figura 4.

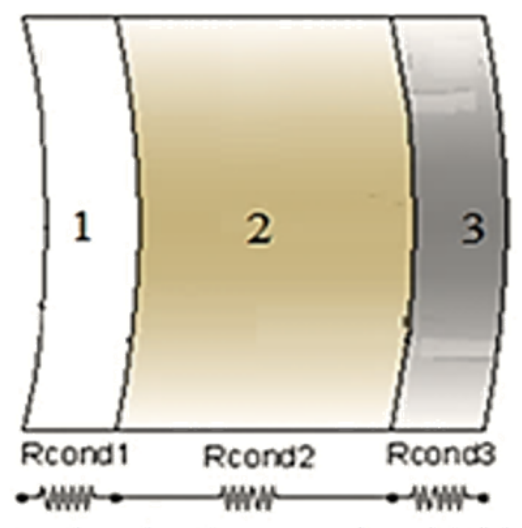

1. Plancha de acero inoxidable

2. Espuma de poliuretano

3. Plancha de acero dulce

Figura 4. Analogía de resistencias para el tanque acumulador multicapa [11].

Las pérdidas se producen en el tanque de almacenamiento por conducción, convección y radiación; $\left.\left(\mathrm{Q}_{\mathrm{p} \text {-tanque }}\right)\right)$ y están dadas por la ecuación (13):

$$
Q_{p-\text { Tanque }}=Q_{p-\text { cond }}+Q_{p-c o n v}+Q_{p-r a d}
$$

Para las pérdidas por conducción en la periferia del tanque se consideran las ecuaciones (14) y (15):

$$
\begin{gathered}
Q_{p-\text { cond }}=\frac{T_{i}-T_{a}}{R_{\text {Total }}} \\
R_{\text {Total }}=R_{\text {cond } 1}+R_{\text {cond } 2}+R_{\text {cond } 3}
\end{gathered}
$$

Donde:

$T_{i}$ es la temperatura en el interior del tanque.

$\mathrm{T}_{\mathrm{a}}$ es la temperatura del ambiente.

$\mathrm{R}_{\text {Total }}$ es la sumatoria de las resistencias térmicas por conducción en cada una de las capas en el tanque acumulador.

Para el cálculo de cada una de las resistencias por conducción se aplica la ecuación (16):

$$
R_{\text {cond }}=\frac{\ln \frac{D}{d}}{2 \times \pi \times L \times K}
$$

Donde:

D es el diámetro mayor de la capa que se esté analizando.

d es el diámetro menor de la capa.

L es la longitud del cilindro.

K es el coeficiente de conductividad térmica de los materiales. 
Para las pérdidas en las tapas del tanque se utiliza de igual manera una analogía con redes de resistencias térmicas ya que el tanque está formado por tres materiales como se muestra en la Figura 5.

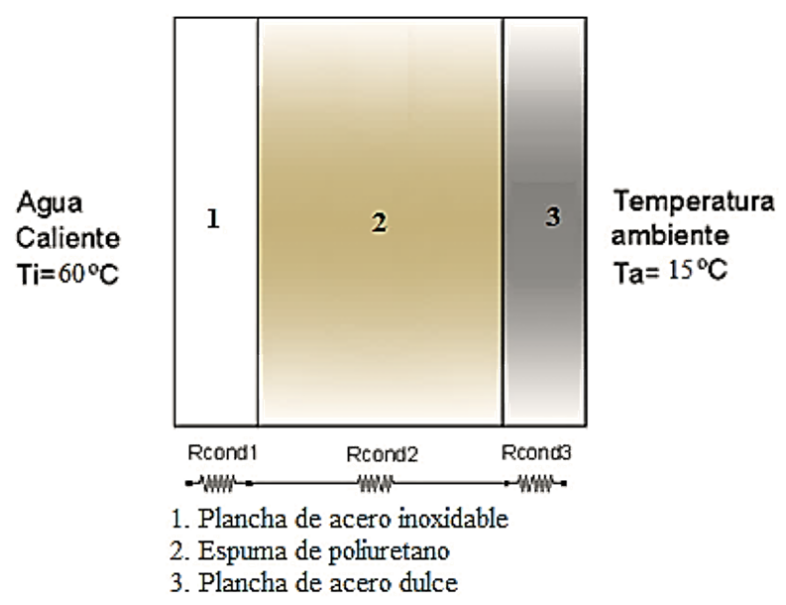

Figura 5. Analogía de resistencias para la tapa del tanque acumulador multicapa [11].

El procedimiento de cálculo es el mismo que se explicó en los párrafos anteriores, pero se modifica la expresión de las resistencias por la ecuación (17) considerando que las superficies son planas.

$$
R=\frac{e}{K \times A}
$$

Donde:

e es el espesor de las diferentes capas de aislamiento. $\mathrm{K}$ es el coeficiente de conductividad térmica.

A es el área de conducción de la capa de aislamiento.

Realizando la suma de la resistencia total del cilindro y la de las tapas se obtiene la resistencia total que reemplazando en la ecuación (14) permite calcular las pérdidas totales de conducción en el tanque $\left(\mathrm{Q}_{p-\text {-cond }}\right)$.

Para las pérdidas por convección en el tanque se utiliza la ecuación (18).

$$
Q_{p-c o n d}=h A_{t}\left(T_{t}-T_{a}\right)
$$

Donde:

h es el coeficiente de transferencia de calor por convección.

$\mathrm{A}_{\mathrm{t}}$ es el área transversal del tanque de almacenamiento. $\mathrm{T}_{\mathrm{t}}$ es la temperatura en la superficie exterior del tanque.

$\mathrm{T}_{\mathrm{a}}$ es la temperatura ambiente.

Para calcular el coeficiente de transferencia de calor por convección se utiliza la ecuación (19):

$$
h=\frac{N_{u} \times K}{D}
$$

\section{Donde:}

$\mathrm{N}_{\mathrm{u}}$ es el número de Nusselt.

$\mathrm{K}$ es el coeficiente de conductividad térmica del aire. $\mathrm{D}$ es el diámetro exterior del tanque de almacenamiento.

Para el cálculo de las pérdidas por radiación en el tanque se utiliza la ecuación (20):

$$
Q_{\text {rad }}=\varepsilon \sigma A_{t}\left(T_{t}^{4}-T_{a}^{4}\right)
$$

Donde:

$\varepsilon$ es la emisividad de la superficie del tanque. $\sigma$ es el coeficiente de Stefan Boltzman.

$\mathrm{A}_{\mathrm{t}}$ es el área transversal del tanque de almacenamiento.

\subsubsection{Caracterización de calentador solar de tubos de vacío con tubo de calor.}

El proceso de cálculo de eficiencia y de pérdidas en el tanque es el mismo que para tubos de vacío; la diferencia para este tipo de calentador está en el sistema de tubos de vacío con tubos de calor en donde interviene un elemento adicional en el intercambio que modificará el valor de eficiencia en el sistema. Para este caso en particular el $U_{l r}$ será ahora $U_{l}$ que es el coeficiente de pérdidas de calor por radiación de los tubos.

El cálculo de $\mathrm{U}_{1}$ para este tipo de calentador se lo realiza mediante la fórmula propuesta por Duffie \& Beckman [13], en la cual relacionan las pérdidas por radiación de la superficie del tubo receptor hacia el tubo de cubierta $\left(\mathrm{h}_{\mathrm{r}, \mathrm{r}-\mathrm{c}}\right)$, las pérdidas por radiación del tubo de cubierta hacia el ambiente $\left(\mathrm{h}_{\mathrm{r}, \mathrm{c}-\mathrm{a}}\right)$ y, además, se consideran las pérdidas entre el tubo de calor hacia el tubo receptor $\left(h_{r, t c-r}\right)$ como se muestra en la ecuación (21):

$$
U_{l}=\left[\frac{A_{r}}{h_{w}+h_{r, c-a} \times A_{c}}+\frac{1}{h_{r, r-c}}+\frac{1}{h_{r, t c-r}}\right]^{-1}
$$

El término que se incorpora considera las pérdidas entre el tubo de calor hacia el tubo receptor $\left(h_{r}, t c-r\right)$ y se calcula con la fórmula (22):

$$
h_{r, t c-r}=\frac{\sigma \times 4 \times T^{3}}{\frac{1-\varepsilon_{1}}{\varepsilon_{1}}+1 \frac{\left(1-\varepsilon_{2}\right) A_{1}}{\varepsilon_{2} A_{2}}}
$$

Donde:

$\varepsilon_{1}$ es la emisivadad del cobre.

$\varepsilon_{2}$ es la emisividad del tubo de vidrio.

$\mathrm{A}_{1}$ es el área del tubo de calor.

$\mathrm{A}_{2}$ es el área del tubo de vidrio.

$\sigma$ es el coeficiente de Stefan Boltzman.

$\mathrm{T}$ es la temperatura del receptor. 


\subsubsection{Determinación práctica de la eficiencia del colector de tubos de vacío y del colec- tor de tubos de vacío con tubo de calor.}

Para los dos tipos de calentadores instalados se realiza el monitoreo de las siguientes variables:

- Temperatura ambiente $\left[{ }^{\circ} \mathrm{C}\right]$

- Temperatura del agua en el tanque acumulador $\left[{ }^{\circ} \mathrm{C}\right]$

- Temperatura de ingreso del agua de la red $\left[{ }^{\circ} \mathrm{C}\right]$

- Volumen de agua [litros]

Con los valores medidos durante un año se calcula la eficiencia del calentador utilizando la fórmula 23:

$$
\eta_{s}=\frac{\dot{m} \times C_{p} \times\left(T_{s}-T_{t}\right)}{Q_{i n c}}
$$

Donde:

m es el flujo másico de agua que circula por el calentador $[\mathrm{kg} / \mathrm{s}]$.

$\mathrm{C}_{\mathrm{p}}$ es el calor específico del agua $[\mathrm{J} / \mathrm{kg} \cdot \mathrm{K}]$.

$\mathrm{T}_{\mathrm{S}}$ es la temperatura de salida del agua $\left[{ }^{\circ} \mathrm{C}\right]$.

$\mathrm{T}_{\mathrm{i}}$ es la temperatura de ingreso del agua $\left[{ }^{\circ} \mathrm{C}\right]$.

$\mathrm{Q}_{\text {inc }}$ es la energía útil proveniente del sol $\left[\mathrm{W} / \mathrm{m}^{2}\right]$.

Finalmente, se comparan los datos medidos con los datos calculados y se realiza la corrección en el análisis teórico.

2.4. Factibilidad de implementación de sistemas solares para obtención de ACS y determinación de disminución de la contaminación ambiental

En este punto se definirá la factibilidad de implementación de sistemas para obtención de ACS considerando la cantidad de energía solar medida durante los años 2014 y 2015 para las parroquias del cantón Cuenca.

Para el análisis se parte de los datos de radiación solar medidos y se calcula en función de la radiación la energía que los dos tipos de calentadores son capaces de aportar para obtener ACS, se compara con la demanda energética determinada en el trabajo de campo, se calcula el porcentaje de aporte que podrían generar estos sistemas y la disminución que se podría dar en emanaciones de $\mathrm{CO}_{2}$ con la implementación de los mismos.

Además, se determina la cantidad de energía que se debería generar con fuentes convencionales complementarias para garantizar una constante dotación de agua caliente en las viviendas.

\section{Resultados y discusión}

En la primera etapa para determinar la muestra se aplica la ecuación (1).

Remplazando los valores se tiene que el tamaño de la muestra es de 531 hogares.

Aplicando la encuesta se obtiene que el $82 \%$ de los hogares del cantón Cuenca utilizan ACS y el $18 \%$ no la utilizan. (Figura 6)

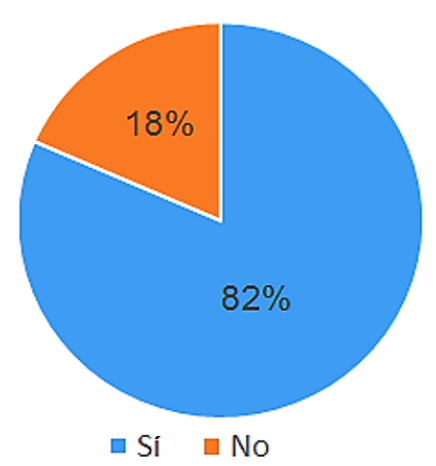

Figura 6. ACS en hogares en el cantón Cuenca.

Quienes no utilizan ACS consideran que es una implementación costosa y la contaminación ambiental que se pueda generar fue considerada como un factor nada importante (Figura 7).

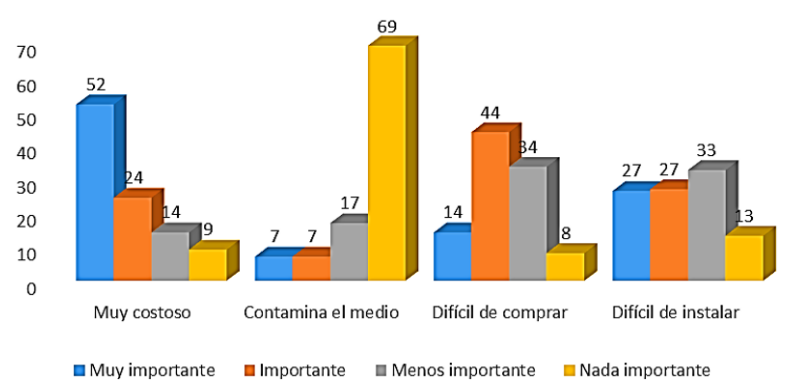

Figura 7. Motivos por los cuales un hogar cuencano no dispone de ACS.

En el cantón Cuenca el número de habitantes por hogar se encuentra comprendido entre 3 y 6 , como se muestra en la Figura 8; la tendencia de la curva está hacia la derecha y se mantiene para la zona urbana y la rural, por lo que el valor considerado de cinco miembros por hogar es una alternativa válida para el análisis. 


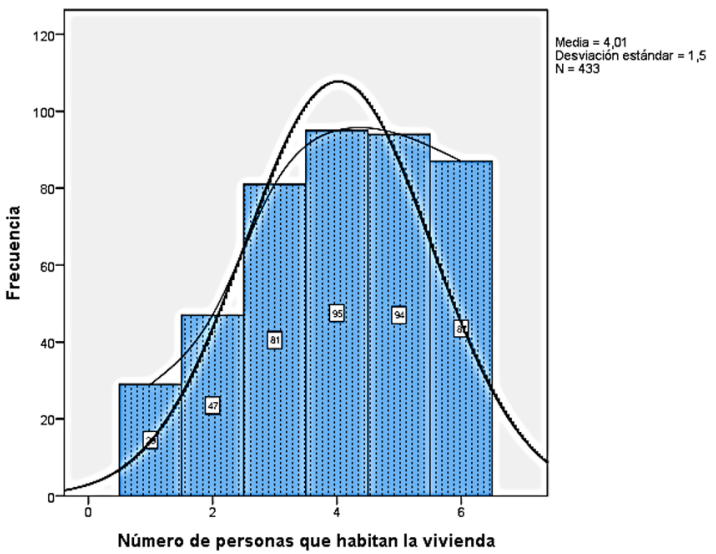

Figura 8. Número de habitantes por vivienda en el cantón Cuenca.

Con respecto a los sistemas utilizados para obtener agua caliente sanitaria, predomina el sistema de calefacción con GLP (Figura 9).

El ACS se utiliza fundamentalmente en ducha, lavamanos y fregadero de cocina, y la frecuencia de uso para los cinco miembros en lavamanos y fregadero de cocina está entre 30 a 45 min diarios y para la ducha está entre 30 y 60 minutos (Figura 10).

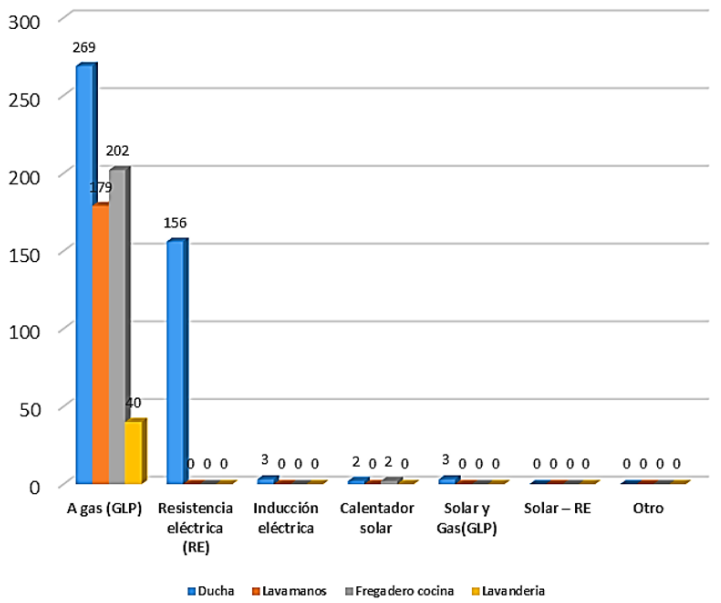

Figura 9. Sistemas utilizados para obtención de ACS.

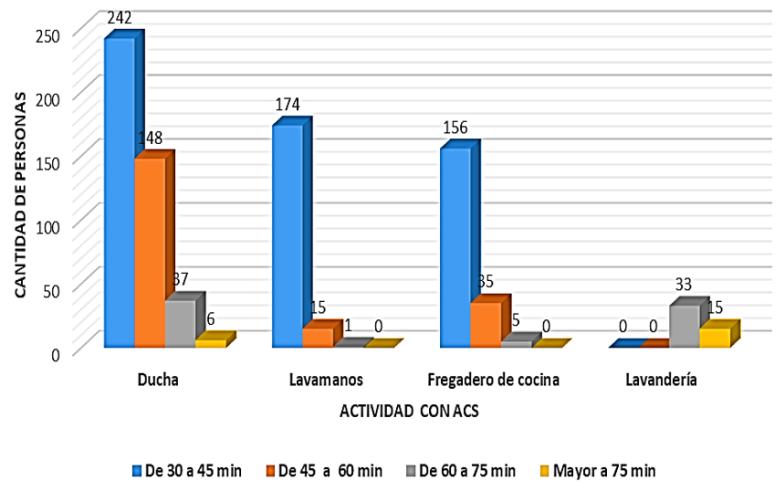

Figura 10. Tipo de actividad con ACS y tiempo empleado.
Los sistemas utilizados actualmente para obtener ACS son eficientes ya que la población los califica como buenos y muy buenos (Figura 11).

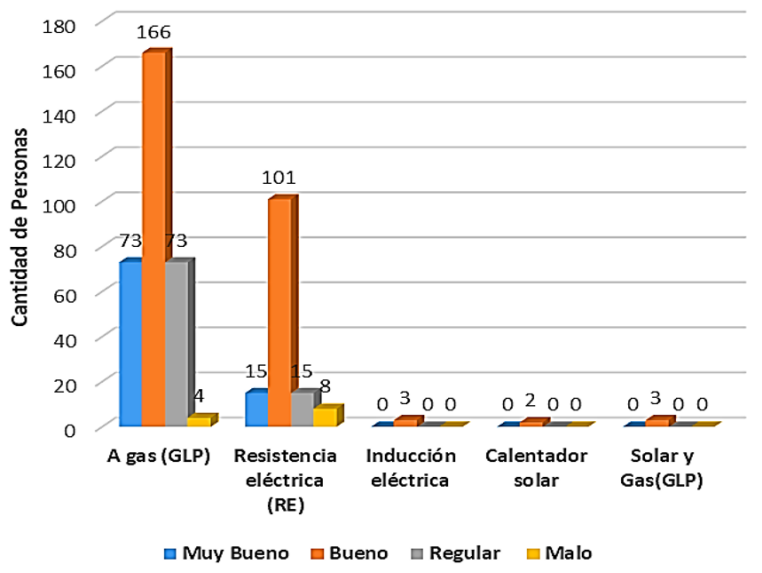

Figura 11. Calificación del sistema de ACS.

A la hora de seleccionar el sistema lo que más importancia tiene es el costo del sistema y lo que menos importante es la contaminación que este pueda generar (Figura 12).

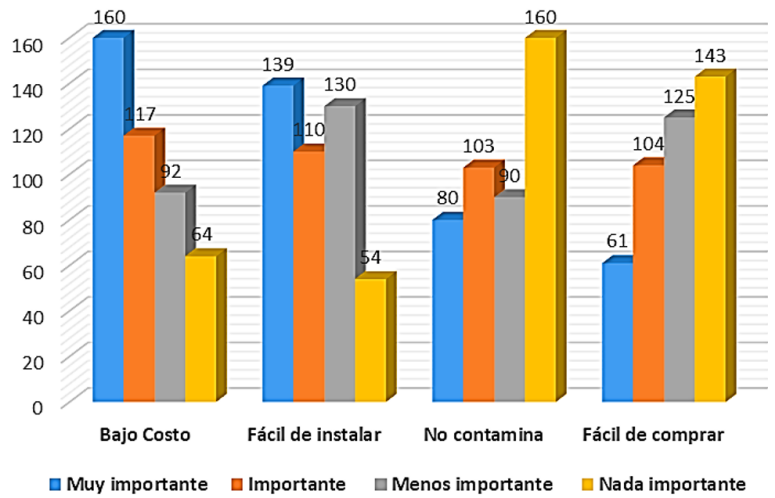

Figura 12. Importancia de parámetros a la hora de escoger un sistema para obtener ACS.

A partir de la pregunta 4 se determina el valor promedio de consumo de ACS, para esto se calcula los valores promedios de la sumatoria de los tiempos de uso para ducha, lavamanos y fregadero de cocina, en lo que corresponde a lavandería se desprecia ya que el porcentaje de usuarios en esta actividad es mínimo.

Con lo antes indicado se determina el consumo de ACS, multiplicando el tiempo total empleado por los miembros del hogar por un consumo promedio de $0,006 \mathrm{~m}^{3} / \min$ [14], y por un factor de coincidencia pues nos todos los miembros se bañan todos los días, o utilizan ACS en el lavamanos o en el fregadero de cocina, estos valores han sido identificados considerando las costumbres de uso de los habitantes del cantón Cuenca; los resultados obtenidos se pueden observar en la Tabla 4. 
Tabla 4. Cálculo del consumo efectivo de ACS por hogar de 5 miembros en el cantón Cuenca

\begin{tabular}{cccccc}
\hline Actividad & $\begin{array}{c}\text { Tiempo } \\
\text { promedio } \\
\text { diario (min) }\end{array}$ & $\begin{array}{c}\text { Factor de } \\
\text { coincidencia }\end{array}$ & $\begin{array}{c}\text { Tiempo diario } \\
\text { efectivo }(\mathbf{m i n})\end{array}$ & $\begin{array}{c}\text { Caudal en } \\
\text { tubería } \\
\left(\mathbf{m}^{3} / \mathbf{m i n}\right)\end{array}$ & $\begin{array}{c}\text { Consumo } \\
\text { efectivo } \\
\left(\mathbf{m}^{3} / \mathbf{d i ́ a}\right)\end{array}$ \\
\hline Ducha & 44 & 0,5 & 22 & 0,006 & 0,13 \\
Lavamanos & 30 & 0,4 & 12 & 0,006 & 0,07 \\
Fregadero de cocina & 20 & 0,4 & 8 & 0,006 & 0,05 \\
& & & & Total & $\mathbf{0 , 2 5}$ \\
\hline
\end{tabular}

Para calcular la demanda de energía mensual utilizamos la ecuación (2). El valor correspondiente a $\mathrm{T}_{\text {uso }}$ es $60{ }^{\circ} \mathrm{C}$, mientras que para $\mathrm{T}_{\text {red }}$ es un promedio de $8{ }^{\circ} \mathrm{C}$, este valor se asume ya que existe una variación entre 6 y $10{ }^{\circ} \mathrm{C}$ en las zonas urbana y rural; el valor de $\mathrm{V}_{\mathrm{ACS}}$ es $0,25 \mathrm{~m}^{3}$ /día, conocidos los valores de los parámetros se procede a remplazar en la expresión considerando los días de cada mes y se determina el valor mensual y anual de energía requerida para producir ACS; los valores calculados se muestran en la Tabla 5.
Con el valor calculado de la demanda de energía mensual y anual requerida para obtener ACS para una familia de 5 miembros se procede a calcular la producción de $\mathrm{CO}_{2}$, multiplicando al valor obtenido por el índice de emisiones que para el GLP genérico es de $0,234 \mathrm{~kg}$ de $\mathrm{CO}_{2} \mathrm{eq} / \mathrm{kWh}$ [7] obteniéndose que se produce $2141,20 \mathrm{~kg}$ de $\mathrm{CO}_{2}$ eq al año en una familia promedio de 5 miembros en el cantón Cuenca como se presenta en la Tabla 5 .

Tabla 5. Demanda de energía mensual y anual para producir ACS en un hogar de 5 miembros en el cantón Cuenca y producción de $\mathrm{CO}_{2}$

\begin{tabular}{|c|c|c|c|c|c|c|c|c|c|}
\hline Mes & Días & $\begin{array}{r}\text { Consumo } \\
\left(\mathrm{m}^{3} / \text { día }\right)\end{array}$ & $\begin{array}{l}\text { Consumo } \\
\text { total }\left(\mathrm{m}^{3}\right) \\
\text { mensual }\end{array}$ & $\begin{array}{l}\text { Tred } \\
\left({ }^{\circ} \mathrm{C}\right)\end{array}$ & $\begin{array}{l}\text { Tuso } \\
\left({ }^{\circ} \mathrm{C}\right)\end{array}$ & $\Delta \mathbf{T}\left({ }^{\circ} \mathbf{C}\right)$ & $\begin{array}{c}\mathrm{D}_{\mathrm{ACS}}(\mathrm{MJ}) \\
\text { por mes }\end{array}$ & $\begin{array}{c}\mathrm{D}_{\mathrm{ACS}}(\mathrm{kWh}) \\
\text { por mes }\end{array}$ & $\begin{array}{c}\text { kg eq } \\
\text { de } \mathrm{CO}_{2}\end{array}$ \\
\hline Enero & 31 & 0,25 & 7,81 & 8 & 60 & 52 & 1700,86 & 472,46 & 181,9 \\
\hline Febrero & 28 & 0,25 & 7,06 & 8 & 60 & 52 & 1536,26 & 426,74 & 164,29 \\
\hline Marzo & 31 & 0,25 & 7,81 & 8 & 60 & 52 & 1700,86 & 472,46 & 181,9 \\
\hline Abril & 30 & 0,25 & 7,56 & 8 & 60 & 52 & 1645,99 & 457,22 & 176,03 \\
\hline Mayo & 31 & 0,25 & 7,81 & 8 & 60 & 52 & 1700,86 & 472,46 & 181,9 \\
\hline Junio & 30 & 0,25 & 7,56 & 8 & 60 & 52 & 1645,99 & 457,22 & 176,03 \\
\hline Julio & 31 & 0,25 & 7,81 & 8 & 60 & 52 & 1700,86 & 472,46 & 181,9 \\
\hline Agosto & 31 & 0,25 & 7,81 & 8 & 60 & 52 & 1700,86 & 472,46 & 181,9 \\
\hline Septiembre & 30 & 0,25 & 7,56 & 8 & 60 & 52 & 1645,99 & 457,22 & 176,03 \\
\hline Octubre & 31 & 0,25 & 7,81 & 8 & 60 & 52 & 1700,86 & 472,46 & 181,9 \\
\hline Noviembre & 30 & 0,25 & 7,56 & 8 & 60 & 52 & 1645,99 & 457,22 & 176,03 \\
\hline Diciembre & 31 & 0,25 & 7,81 & 8 & 60 & 52 & 1700,86 & 472,46 & 181,9 \\
\hline \multicolumn{7}{|c|}{ Demanda por año } & 20026,25 & 5562,85 & 2141,7 \\
\hline
\end{tabular}

Considerando los datos anteriores y con 114553 hogares formados por 5 miembros se produciría un aporte a la contaminación de $245338160 \mathrm{~kg}$ eq de $\mathrm{CO}_{2}$ o 245338 t eq de $\mathrm{CO}_{2}$.

Con respecto al monitoreo, procesamiento y análisis de radiación solar global, para este proceso se consideran los datos de radiación medidos por un grupo de 16 estaciones meteorológicas instaladas en el cantón Cuenca según la Tabla 6.

Se realiza la complementación de datos faltantes usando correlación de Angström - Page para los datos de las estaciones mostradas en la Tabla 7.

Se obtienen las tablas complementadas; como ejemplo se presenta para la estación de la parroquia rural de Chaucha la Tabla 8.
Tabla 6. Datos de estaciones meteorológicas

\begin{tabular}{ccc}
\hline $\begin{array}{c}\text { Estación } \\
\text { meteoorológica }\end{array}$ & Coordenadas & $\begin{array}{c}\text { Elevación } \\
(\mathbf{m} \text { s. n. m. })\end{array}$ \\
\hline Quingeo & $729057 ; 9664602$ & 2893 \\
Molleturo & $679708 ; 9692232$ & 3530 \\
Baños & $712899 ; 9672817$ & 3062 \\
CTS & $720504 ; 9677509$ & 2561 \\
UPS & $723584 ; 9680788$ & 2556 \\
Tixán & $723017 ; 9686678$ & 2725 \\
Cumbe & $719190 ; 9656242$ & 3179 \\
Sayausí & $715974 ; 9681200$ & 2727 \\
Nulti & $729704 ; 9682466$ & 2601 \\
San Joaquín & $714405 ; 9680807$ & 2764 \\
Llacao & $730418 ; 9685180$ & 2542 \\
Santa Ana & $730085 ; 9672006$ & 2651 \\
Chaucha & $672859 ; 9678690$ & 1929 \\
Turi & $721103 ; 9674971$ & 2768 \\
Sinincay & $722340 ; 9685283$ & 2696 \\
Victoria del & $713645 ; 9659192$ & 2665 \\
Portete & & \\
& &
\end{tabular}


Tabla 7. Estaciones meteorológicas con datos faltantes

\begin{tabular}{cccc}
\hline Estación & Año & Mes & $\begin{array}{c}\text { Días con datos } \\
\text { faltantes }\end{array}$ \\
\hline \multirow{2}{*}{ CTS } & 2014 & enero & $1-9$ \\
& 2014 & marzo & $19-24$ \\
Chaucha & 2014 & noviembre & $27-30$ \\
Cumbe & 2014 & diciembre & $9-15$ \\
Irquis & 2014 & enero & $1-8$ \\
Santa Ana & 2014 & enero & $1-3$ \\
Turi & 2014 & enero & $1-3$ \\
UPS & 2014 & enero & $1-7$ \\
\hline
\end{tabular}

Tabla 8. Datos faltantes de radiación complementados en el mes de diciembre 2014 para la estación de Chaucha

\begin{tabular}{cccccccccc}
\hline \multirow{2}{*}{ Hora/Día } & $\mathbf{8}$ & $\mathbf{9}$ & $\mathbf{1 0}$ & $\mathbf{1 1}$ & $\mathbf{1 2}$ & $\mathbf{1 3}$ & $\mathbf{1 4}$ & $\mathbf{1 5}$ & $\mathbf{1 6}$ \\
\hline $01 \mathrm{H} 00$ & 0 & 0 & 0 & 0 & 0 & 0 & 0 & 0 & 0 \\
$02 \mathrm{H} 00$ & 0 & 0 & 0 & 0 & 0 & 0 & 0 & 0 & 0 \\
$03 \mathrm{H} 00$ & 0 & 0 & 0 & 0 & 0 & 0 & 0 & 0 & 0 \\
$04 \mathrm{H} 00$ & 0 & 0 & 0 & 0 & 0 & 0 & 0 & 0 & 0 \\
$05 \mathrm{H} 00$ & 0 & 0 & 0 & 0 & 0 & 0 & 0 & 0 & 0 \\
$06 \mathrm{H} 00$ & 0 & 0 & 0 & 0 & 0 & 0 & 0 & 0 & 0 \\
$07 \mathrm{H} 00$ & 13 & 7 & 13 & 13 & 24 & 9 & 14 & 3 & 5 \\
$08 \mathrm{H} 00$ & 59 & 72 & 67 & 81 & 112 & 89 & 72 & 64 & 76 \\
$09 \mathrm{H} 00$ & 123 & 255 & 267 & 495 & 292 & 217 & 151 & 305 & 279 \\
$10 \mathrm{H} 00$ & 569 & 309 & 500 & 739 & 331 & 504 & 191 & 633 & 465 \\
$11 \mathrm{H} 00$ & 955 & 355 & 719 & 941 & 570 & 719 & 387 & 1071 & 902 \\
$12 \mathrm{H} 00$ & 1123 & 569 & 940 & 1085 & 923 & 783 & 555 & 1054 & 1187 \\
$13 \mathrm{H} 00$ & 1114 & 453 & 922 & 1129 & 798 & 536 & 651 & 1063 & 1022 \\
$14 \mathrm{H} 00$ & 975 & 224 & 670 & 1128 & 1004 & 664 & 419 & 325 & 686 \\
$15 \mathrm{H} 00$ & 1059 & 67 & 226 & 432 & 399 & 394 & 790 & 69 & 216 \\
$16 \mathrm{H} 00$ & 173 & 57 & 96 & 89 & 383 & 389 & 241 & 78 & 86 \\
$17 \mathrm{H} 00$ & 50 & 85 & 31 & 90 & 184 & 177 & 68 & 51 & 57 \\
$18 \mathrm{H} 00$ & 11 & 47 & 6 & 54 & 8 & 71 & 62 & 53 & 73 \\
$19 \mathrm{H} 00$ & 1 & 4 & 2 & 4 & 1 & 7 & 6 & 5 & 9 \\
$20 \mathrm{H} 00$ & 0 & 0 & 0 & 0 & 0 & 0 & 0 & 0 & 0 \\
$21 \mathrm{H} 00$ & 0 & 0 & 0 & 0 & 0 & 0 & 0 & 0 & 0 \\
$22 \mathrm{H} 00$ & 0 & 0 & 0 & 0 & 0 & 0 & 0 & 0 & 0 \\
$23 \mathrm{H} 00$ & 0 & 0 & 0 & 0 & 0 & 0 & 0 & 0 & 0 \\
$24 \mathrm{H} 00$ & 0 & 0 & 0 & 0 & 0 & 0 & 0 & 0 & 0 \\
\hline & & & & & & & & &
\end{tabular}

Con los datos complementados se calcula la radiación global total diaria, mensual y anual para cada estación (Figura 12, ejemplo estación de Chaucha).

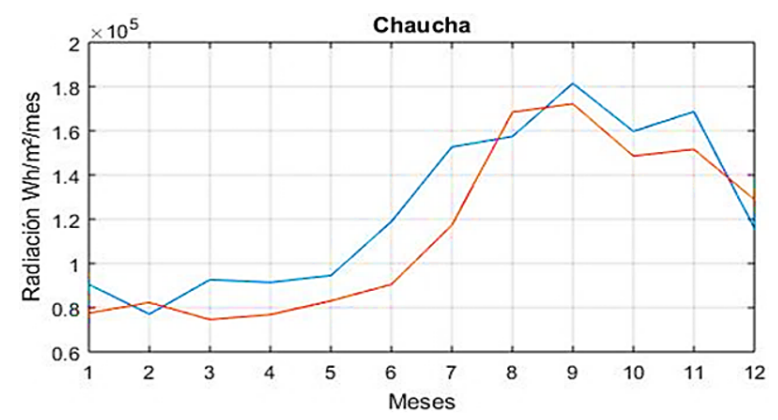

Figura 13. Radiación solar global - Sector Chaucha

Con los promedios de todos los datos medidos en las 16 estaciones se obtiene la curva promedio de radiación solar global para el cantón Cuenca como se muestra en la Figura 14.
Radiación global cantón Cuenca 2014-2015

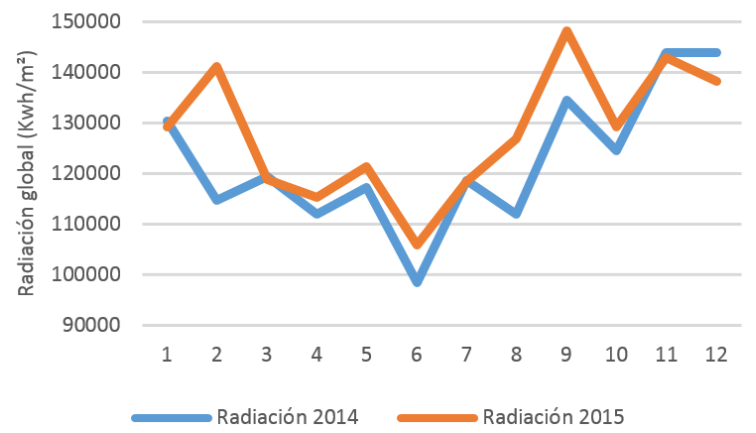

Figura 14. Radiación global - cantón Cuenca 2014-2015

Los calentadores instalados tienen las siguientes características (Figura 15):
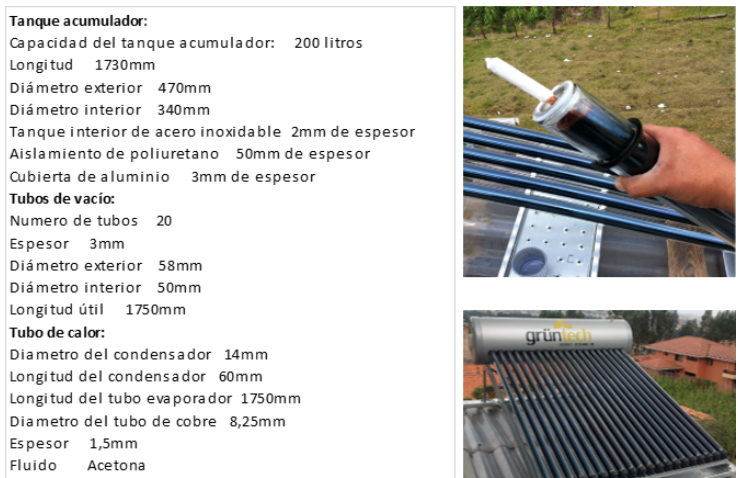

Figura 15. Características de calentadores solares instalados

Se calcula la eficiencia de los colectores solares tanto de manera teórica como práctica y se compara los valores llegando a determinar que el promedio de la diferencia porcentual para el calentador de tubos de vacío evacuados simple es del $5 \%$ y el de tubos de vacío con tubos de calor es del $2 \%$; (Figura 16) con estos valores se corrige el análisis y se determina la eficiencia real en los colectores quedando para el de tubos de vacío es de $74 \%$ y el de tubos de vacío con tubos de calor es de $80 \%$.
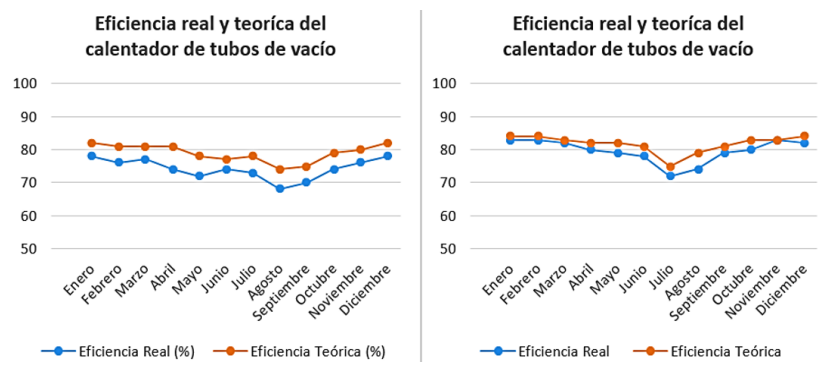

Figura 16. Eficiencia real y teórica de calentadores solares

Con los datos de radiación complementados y definidas las eficiencias de los calentadores se calculan 
la energía de pérdida y la energía útil que se empleará que debe ser cubierta con sistemas auxiliares para cada para la obtención de ACS y se comparan con la de- tipo de calentador (Tablas 9, 10). Para el cálculo se manda de energía para establecer la cantidad faltante considera 9 horas efectivas de sol diarias.

Tabla 9. Análisis energético para el calentador de tubos de vacío

\begin{tabular}{|c|c|c|c|c|c|c|c|c|c|c|c|c|c|c|}
\hline \multirow[t]{2}{*}{ Estación } & \multicolumn{2}{|c|}{$\begin{array}{c}\text { Irradiancia } \\
\text { promedio } \\
\mathrm{kWh} / \mathrm{m}^{2}\end{array}$} & \multicolumn{2}{|c|}{$\begin{array}{c}\text { Energía } \\
\text { incidente } \\
\mathrm{kWh}\end{array}$} & \multicolumn{2}{|c|}{$\begin{array}{c}\text { Energía } \\
\text { perdida } \\
\text { kWh }\end{array}$} & \multicolumn{2}{|c|}{$\begin{array}{c}\text { Energía } \\
\text { útil } \\
\mathrm{kWh}\end{array}$} & \multicolumn{2}{|c|}{$\begin{array}{c}\text { Demanda } \\
\text { energía } \\
\text { kWh }\end{array}$} & \multicolumn{2}{|c|}{$\begin{array}{c}\text { Energía } \\
\text { faltante } \\
\mathrm{kWh}\end{array}$} & \multicolumn{2}{|c|}{$\begin{array}{c}\text { Energía } \\
\text { faltante } \\
\mathrm{kg}(\mathrm{GLP})\end{array}$} \\
\hline & 2014 & 2015 & 2014 & 2015 & 2014 & 2015 & 2014,00 & 2015,00 & 2014 & 2015 & 2014 & 2015 & 2014 & 2015 \\
\hline Baños & 1359,11 & 1349,73 & 2729,67 & 2710,82 & 709,71 & 704,81 & 2019,96 & 2006,00 & 5562,85 & 5562,85 & 3542,89 & 3556,85 & 280,32 & 281,42 \\
\hline Chaucha & 1454,31 & 1372,09 & 2920,86 & 2755,73 & 759,42 & 716,49 & 2161,43 & 2039,24 & 5562,85 & 5562,85 & 3401,42 & 3523,61 & 269,12 & 278,79 \\
\hline CTS & 1527,98 & 1551,79 & 3068,82 & 3116,64 & 797,89 & 810,33 & 2270,93 & 2306,31 & 5562,85 & 5562,85 & 3291,92 & 3256,54 & 260,46 & 257,66 \\
\hline Cumbe & 1457,39 & 1525,81 & 2927,04 & 3064,46 & 761,03 & 796,76 & 2166,01 & 2267,70 & 5562,85 & 5562,85 & 3396,84 & 3295,15 & 268,76 & 260,72 \\
\hline Irquis & 1466,93 & 1509,14 & 2946,20 & 3030,99 & 766,01 & 788,06 & 2180,19 & 2242,93 & 5562,85 & 5562,85 & 3382,66 & 3319,92 & 267,64 & 262,67 \\
\hline Llacao & 1609,21 & 1665,49 & 3231,97 & 3345,01 & 840,31 & 869,70 & 2391,66 & 2475,31 & 5562,85 & 5562,85 & 3171,19 & 3087,54 & 250,91 & 244,29 \\
\hline Molleturo & 1823,05 & 1968,17 & 3661,44 & 3952,91 & 951,97 & 1027,76 & 2709,47 & 2925,15 & 5562,85 & 5562,85 & 2853,38 & 2637,70 & 225,76 & 208,70 \\
\hline Nulti & 1691,23 & 1781,70 & 3396,70 & 3578,40 & 883,14 & 930,38 & 2513,56 & 2648,01 & 5562,85 & 5562,85 & 3049,29 & 2914,84 & 241,26 & 230,62 \\
\hline Quingeo & 1472,95 & 1570,64 & 2958,31 & 3154,51 & 769,16 & 820,17 & 2189,15 & 2334,34 & 5562,85 & 5562,85 & 3373,70 & 3228,51 & 266,93 & 255,44 \\
\hline San Joaquín & 1414,30 & 1364,51 & 2840,51 & 2740,51 & 738,53 & 712,53 & 2101,98 & 2027,98 & 5562,85 & 5562,85 & 3460,87 & 3534,87 & 273,83 & 279,68 \\
\hline Santa Ana & 1431,65 & 1482,57 & 2875,36 & 2977,62 & 747,59 & 774,18 & 2127,76 & 2203,44 & 5562,85 & 5562,85 & 3435,09 & 3359,41 & 271,79 & 265,80 \\
\hline Sayausí & 1435,70 & 1440,54 & 2883,49 & 2893,20 & 749,71 & 752,23 & 2133,78 & 2140,97 & 5562,85 & 5562,85 & 3429,07 & 3421,88 & 271,31 & 270,74 \\
\hline Sinincay & 1523,17 & 1552,78 & 3059,17 & 3118,64 & 795,38 & 810,85 & 2263,79 & 2307,79 & 5562,85 & 5562,85 & 3299,06 & 3255,06 & 261,02 & 257,54 \\
\hline Tixán & 1462,79 & 1472,53 & 2937,90 & 2957,45 & 763,85 & 768,94 & 2174,04 & 2188,51 & 5562,85 & 5562,85 & 3388,81 & 3374,34 & 268,13 & 266,98 \\
\hline Turi & 1441,54 & 1515,15 & 2895,23 & 3043,06 & 752,76 & 791,20 & 2142,47 & 2251,86 & 5562,85 & 5562,85 & 3420,38 & 3310,99 & 270,62 & 261,97 \\
\hline \multirow[t]{3}{*}{ UPS } & 1640,31 & 1679,48 & 3294,43 & 3373,09 & 856,55 & 877,00 & 2437,88 & 2496,09 & 5562,85 & 5562,85 & 3124,97 & 3066,76 & 247,25 & 242,64 \\
\hline & & & & & & & & & \multirow{2}{*}{\multicolumn{2}{|c|}{ Valores promedio }} & 3313,85 & 3259,00 & 262,19 & 257,85 \\
\hline & & & & & & & & & & & \multicolumn{2}{|c|}{3286,42} & \multicolumn{2}{|c|}{260,02} \\
\hline
\end{tabular}

Tabla 10. Análisis energético para el calentador de tubos de vacío con tubos de calor

\begin{tabular}{|c|c|c|c|c|c|c|c|c|c|c|c|c|c|c|}
\hline \multirow[t]{2}{*}{ Estación } & \multicolumn{2}{|c|}{$\begin{array}{l}\text { Irradiancia } \\
\text { promedio } \\
\mathrm{kWh} / \mathrm{m}^{2}\end{array}$} & \multicolumn{2}{|c|}{$\begin{array}{c}\text { Energía } \\
\text { incidente } \\
\mathrm{kWh}\end{array}$} & \multicolumn{2}{|c|}{$\begin{array}{c}\text { Energía } \\
\text { perdida } \\
\text { kWh }\end{array}$} & \multicolumn{2}{|c|}{$\begin{array}{c}\text { Energía } \\
\text { útil } \\
\text { kWh }\end{array}$} & \multicolumn{2}{|c|}{$\begin{array}{c}\text { Demanda } \\
\text { energía } \\
\text { kWh }\end{array}$} & \multicolumn{2}{|c|}{$\begin{array}{c}\text { Energía } \\
\text { faltante } \\
\mathrm{kWh}\end{array}$} & \multicolumn{2}{|c|}{$\begin{array}{c}\text { Energía } \\
\text { faltante } \\
\mathrm{kg}(\mathrm{GLP})\end{array}$} \\
\hline & 2014 & 2015 & 2014 & 2015 & 2014 & 2015 & 2014,00 & 2015,00 & 2014 & 2015 & 2014 & 2015 & 2014 & 2015 \\
\hline Baños & 1359,11 & 1349,73 & 2729,67 & 2710,82 & 545,93 & 542,16 & 2183,74 & 2168,65 & 5562,85 & 5562,85 & 3379,11 & 3394,20 & 267,36 & 268,55 \\
\hline Chaucha & 1454,31 & 1372,09 & 2920,86 & 2755,73 & 584,17 & 551,15 & 2336,69 & 2204,58 & 5562,85 & 5562,85 & 3226,16 & 3358,27 & 255,26 & 265,71 \\
\hline CTS & 1527,98 & 1551,79 & 3068,82 & 3116,64 & 613,76 & 623,33 & 2455,06 & 2493,31 & 5562,85 & 5562,85 & 3107,79 & 3069,54 & 245,89 & 242,86 \\
\hline Cumbe & 1457,39 & 1525,81 & 2927,04 & 3064,46 & 585,41 & 612,89 & 2341,64 & 2451,57 & 5562,85 & 5562,85 & 3221,21 & 3111,28 & 254,87 & 246,17 \\
\hline Irquis & 1466,93 & 1509,14 & 2946,20 & 3030,99 & 589,24 & 606,20 & 2356,96 & 2424,79 & 5562,85 & 5562,85 & 3205,89 & 3138,06 & 253,65 & 248,29 \\
\hline Llacao & 1609,21 & 1665,49 & 3231,97 & 3345,01 & 646,39 & 669,00 & 2585,58 & 2676,01 & 5562,85 & 5562,85 & 2977,27 & 2886,84 & 235,56 & 228,41 \\
\hline Molleturo & 1823,05 & 1968,17 & 3661,44 & 3952,91 & 732,29 & 790,58 & 2929,15 & 3162,33 & 5562,85 & 5562,85 & 2633,70 & 2400,52 & 208,38 & 189,93 \\
\hline Nulti & 1691,23 & 1781,70 & 3396,70 & 3578,40 & 679,34 & 715,68 & 2717,36 & 2862,72 & 5562,85 & 5562,85 & 2845,49 & 2700,13 & 225,14 & 213,64 \\
\hline Quingeo & 1472,95 & 1570,64 & 2958,31 & 3154,51 & 591,66 & 630,90 & 2366,65 & & 5562,85 & 5562,85 & 3196,20 & 3039,24 & 252,89 & 240,47 \\
\hline San Joaquín & 1414,30 & 1364,51 & 2840,51 & 2740,51 & 568,10 & & 2272,41 & & 5562,85 & & 3290,44 & 3370,44 & 260,34 & 266,67 \\
\hline Santa Ana & 1431,65 & 1482,57 & 2875,36 & 2977,62 & 575,07 & 595,52 & 2300,29 & 2382,09 & 5562,85 & 5562,85 & 3262,56 & 3180,76 & 258,14 & 251,66 \\
\hline Sayausí & 1435,70 & 1440,54 & 2883,49 & 2893,20 & 576,70 & 578,64 & 2306,79 & 2314,56 & 5562,85 & 5562,85 & 3256,06 & 3248,29 & 257,62 & 257,01 \\
\hline Sinincay & 1523,17 & 1552,78 & 3059,17 & 3118,64 & 611,83 & 623,73 & 2447,34 & 2494,91 & 5562,85 & 5562,85 & 3115,51 & 3067,94 & 246,50 & 242,74 \\
\hline Tixán & 1462,79 & 1472,53 & 2937,90 & 2957,45 & 587,58 & 591,49 & 2350,32 & 2365,96 & 5562,85 & 5562,85 & 3212,53 & 3196,89 & 254,18 & 252,94 \\
\hline Turi & 1441,54 & 1515,15 & 2895,23 & 3043,06 & 579,05 & 608,61 & 2316,18 & 2434,45 & 5562,85 & 5562,85 & 3246,67 & 3128,40 & 256,88 & 247,52 \\
\hline \multirow[t]{3}{*}{ UPS } & 1640,31 & 1679,48 & 3294,43 & 3373,09 & 658,89 & 674,62 & 2635,54 & 2698,47 & \multirow{3}{*}{\multicolumn{2}{|c|}{$\begin{array}{c}5562,85 \quad 5562,85 \\
\text { Valores promedio }\end{array}$}} & 2927,31 & 2864,38 & 231,61 & 226,63 \\
\hline & & & & & & & & & & & 3131,49 & 3072,20 & 247,77 & 243,07 \\
\hline & & & & & & & & & & & \multicolumn{2}{|c|}{3101,85} & \multicolumn{2}{|c|}{245,42} \\
\hline
\end{tabular}

Con los valores establecidos se determina que con el calentador solar de tubos de vacío existe una cobertura promedio con energía solar del 40,92\% y para el calentador de tubos de vacío con tubo de calor el $44,23 \%$, esto implica una reducción promedio en el

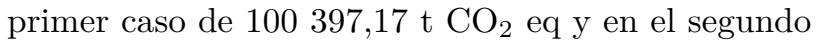
caso de 108537,24 t $\mathrm{CO}_{2}$ eq considerando que al utilizar calentadores a base de GLP se generan 245337,84 t $\mathrm{CO}_{2}$ eq al año (Figura 17).

Los valores de cobertura y disminución de contaminación tendrán una ligera variación dependiendo de la zona en la que están ubicadas las mismas que están adscritas a las mediciones de las 16 estaciones meteorológicas instaladas [15].
Disminución de emisiones de $\mathrm{CO} 2$

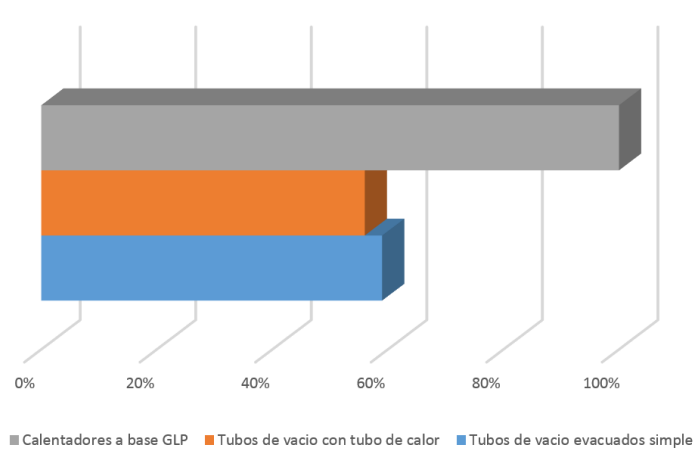

Figura 17. Disminución de emisiones de $\mathrm{CO}_{2}$ en la obtención de ACS con calentadores híbridos con tecnología solar respecto a calentadores a base de GLP 


\section{Conclusiones}

En el cantón Cuenca para obtener ACS sus habitantes utilizan sistemas a base de GLP, provocando una contaminación aproximada de 245337 t $\mathrm{CO}_{2}$ eq.

La cantidad de agua caliente utilizada para las diferentes actividades que corresponde a $50 \mathrm{l}$ por persona al día, es excesiva comparándola con los 301 diarios que se utiliza en España [16,17] de acuerdo con las ordenanzas impuestas en ese país.

La radiación promedio en el cantón Cuenca está entre $1359 \mathrm{kWh} / \mathrm{m}^{2}$ y $1968 \mathrm{kWh} / \mathrm{m}^{2}$ dependiendo de las condiciones geográficas de cada sector, utilizando calentadores solares de tubos de vacío se puede generar un aporte energético para obtener ACS que va desde el $40,92 \%$ al $44,23 \%$.

Para garantizar una dotación constante de ACS los calentadores deben ser híbridos, es decir, deben trabajar con energía solar y tener un sistema auxiliar que puede ser a base de GLP o resistencia eléctrica.

Si se realiza la implementación de sistemas híbridos con tecnología solar se obtiene una disminución de contaminación de $\mathrm{CO}_{2}$ que está alrededor del $44 \%$, esto implica dejar de emanar al ambiente 108 537,24 tn $\mathrm{CO}_{2}$ eq al año.

\section{Referencias}

[1] B. Veritas, Manual para la formación en medio ambiente, S. LEX NOVA, Ed., 2008. [Online]. Available: https://goo.gl/vLX6Hy

[2] Eurobserv'er. (2017) Solar thermal and concentrated solar power barometer 2017. [Online]. Available: https://goo.gl/2XFMc8

[3] F. Entrena, Determinación del potencial solar UF0212, ic editorial, Ed., 2013. [Online]. Available: https://goo.gl/9PsoEw

[4] R. Hernández Sampieri, C. Fernández Collado, and P. Baptista Lucio, Metodología de la Investigación, $5^{\text {th }}$ ed., M. G. Hill, Ed., 2010. [Online]. Available: https://goo.gl/1zETzt

[5] G. Ríos Jiménez. (2016) Métodos para calcular la población futura. [Online]. Available: https://goo.gl/WCH7wy

[6] INEC. (2010) Resultados del censo 2010. [Online]. Available: https://goo.gl/1BC66V

[7] Gobierno de Aragón. (1967) Cálculo de emisiones. [Online]. Available: https://goo.gl/yPfTVh

[8] UPS - INER. (2013) Métodos para el control de calidad y compementación de datos faltantes en parámetros meteorológicos relacionados con la utilización de energías renovables. [Online]. Available: https://goo.gl/1JLb9m

[9] I. Korkut, M. Kasap, I. Ciftci, and U. Seker, Cultivos energéticos alternativos, 2009, ch. Determinación de emplazamientos adecuados para estaciones meteorológicas en la provincia de Imbabura utilizando sistemas de información geográficos, pp. 75-87. [Online]. Available: https://goo.gl/a8b2ZB

[10] J. Martínez Aguirre and J. Asitimbay Chávez, "Caracterización y análisis estadístico de la radiación solar directa para aplicaciones de media y alta temperatura en áreas circundantes al OCP," Master's thesis, Universidad de las Fuerzas Armadas, 2015. [Online]. Available: https://goo.gl/X4RgVK

[11] J. Jiménez Patiño, H. Llivichuzca Chacha, and J. Calle Sigüencia, "Diseño, implementación y monitoreo de calentadores híbridos con energía solar-GLP y energía solar-calentadores eléctricos para la obtención de agua caliente sanitaria." Tesis de grado, Universidad Politécnica Salesiana, Ecuador, 2014. [Online]. Available: https://goo.gl/4kV4w8

[12] Sitiosolar. (2014) Los colectores solares de tubo de vacío. [Online]. Available: https://goo.gl/EnpH8n

[13] J. A. Duffie and W. A. Beckman, Solar Engineering of Thermal Processes, I. John Wiley \& Sons, Ed., 2013. [Online]. Available: https://goo.gl/rjjMtJ

[14] Afta-asociation. Dimensionado de instalaciones. [Online]. Available: https://goo.gl/pnQpzi

[15] O. Delgado, "Plan de ordenamiento y desarrollo territorial del cantón Cuenca," in Memorias del II Congreso Binacional de Investigación, Ciencia y Tecnología de las Universidades, 2013, pp. 721-729. [Online]. Available: https://goo.gl/S3WixM

[16] D. Fuentes Cantero, "Instalación de colectores solares para suministro de ACS en Valencia." Proyecto de Fin de Carrera, Universidad Carlos III de Madrid, España, 2009. [Online]. Available: https://goo.gl/UTh93M

[17] ATECYR - IDAE, Guía técnica agua caliente sanitaria central, Instituto para la Diversificación y Ahorro de la Energía Std., 2015. [Online]. Available: https://goo.gl/HCKYtz 


\title{
CUANTIFICACIÓN DE LA OPACIDAD EN MOTORES ELECTRÓNICOS DIÉSEL USANDO DIÉSEL Y BIODIÉSEL
}

\section{QUANTIFICATION OF OPACITY IN DIESEL ELECTRONIC ENGINES USING DIESEL AND BIODIESEL}

\author{
Carlos Mafla Yépez ${ }^{1, *}$, Rommel Imbaquingo Navarrete ${ }^{1}$, Jorge Melo Obando ${ }^{1}$, \\ Ignacio Benavides Cevallos ${ }^{1}$, Erik Hernández Rueda ${ }^{1}$
}

\section{Resumen}

El presente estudio tiene la finalidad de determinar los niveles de opacidad con el uso de diésel fósil como de biodiésel a base de higuerilla al $15 \%$ (B15). Para la determinación de la opacidad se utilizó un banco de pruebas de motores diésel electrónico, se realizó las mediciones de opacidad usando un opacímetro debidamente calibrado. El opacímetro consta de una sonda la cual se le instala en el extremo del tubo de escape, captando los gases y arrojando valores propios por cada prueba, considerando los tipos de combustibles fósil o biocombustible y el régimen del motor, sometiendo a varias revoluciones por minuto que van de 1200 a 3000 por cada combustible. Al concluir las pruebas el opacímetro permitió obtener una opacidad de $11,1 \%$ con diésel fósil y 0,386 con biodiésel B15 obteniendo una reducción del $96 \%$ de opacidad, a la vez se obtuvo un promedio de opacidad de las pruebas por cada tipo de combustible siendo este valor el que se comparará con las normas nacionales e internacionales concernientes a este estudio. Con estos resultados se contribuye a la mejor conservación del ambiente ya que se emana menor cantidad de hollín, de igual manera se disminuye las enfermedades respiratorias y de corazón de las personas según la organización mundial de la salud.

Palabras clave: biodiésel, B15, diésel fósil, higuerilla, opacidad, RPM.

\section{Abstract}

The present study has the purpose of determining the levels of opacity with the use of diesel as biodiesel based on castor oil at 15\% (B15). For the determination of the opacity an electronic diesel engine test bench was used, the opacity measurements were made using a duly calibrated opacimeter. The Opacimeter consists of a probe which is installed at the end of the exhaust pipe, which captures the gases and yields values for each test, considering the types of fossil or biofuel fuels and engine speed, subjecting several RPMs that go from 1200 to 3000 for each fuel. At the end of the tests, the opacimeter allowed to obtain values that indicate the difference in opacity and to make a comparative analysis of the use of diesel and biodiesel. At the same time, an average opacity of the tests was obtained for each type of fuel, this value being the same that will be compared with the national standard concerning this study. From the tests carried out, a $96 \%$ reduction in the opacity of the engine could be evidenced with the use of B15 biodiesel from Higuerilla oil compared to diesel.

Keywords: biodiesel, B15, fossil diesel, castor oil, opacity, RPM.

\footnotetext{
${ }_{1, *}$ Grupo de investigación en motores de combustión interna con biocombustible (BICER), Universidad Técnica del Norte, Ibarra - Ecuador. Autor para correspondencia c cnmafla@utn.edu.ec, (D) http://orcid.org/0000-0003-3704-8676

(D) https://orcid.org/0000-0001-7140-3374, (D) https://orcid.org/0000-0003-0546-6988

(D) https://orcid.org/0000-0003-3332-8523, (D) https://orcid.org/0000-0003-1969-9619
}

Recibido: 15-11-2017, aprobado tras revisión: 28-12-2017

Forma sugerida de citación: Mafla, C.; Imbaquingo, R.; Melo, J.; Benavides, I.; Hernández, E. (2018). «Cuantificación de la opacidad en motores electrónicos diésel usando diésel y biodiésel». INGEniUs. N. ${ }^{\circ} 19$, (enero-junio). pp. 102-107. DOI: https://doi.org/10.17163/ings.n19.2018.10. 


\section{Introducción}

Las emisiones de gases de efecto invernadero (GEI) $\left(\mathrm{NO}_{\mathrm{x}}, \mathrm{CO}_{2}, \mathrm{CO}, \mathrm{HC}\right)$ son los causantes de provocar el calentamiento global, el deterioro del ambiente y la salud de los seres humanos [1]. Según la Organización Mundial del Medio Ambiente, la industria automotriz es la causante del $15 \%$ de la contaminación del ambiente a nivel mundial. Algo muy importante y que no se debe obviar al momento de usar combustibles fósiles es su potencial de generación de emisiones contaminantes, entre los cuales se encuentran el dióxido de carbono $\left(\mathrm{CO}_{2}\right)$, principal gas causante del efecto invernadero; monóxido de carbono $(\mathrm{CO})$, que tiene efectos letales sobre el hombre; óxidos de nitrógeno $\left(\mathrm{NO}_{x}\right)$ e hidrocarburos inquemados o parcialmente quemados $(\mathrm{HC})$, principales formadores de esmog fotoquímico en presencia de luz; emisiones sulfurosas $\left(\mathrm{SO}_{x}\right)$, causantes de la lluvia ácida, generador de sulfatos, y material particulado, causante de enfermedades respiratorias y cáncer [2].

Además, se conoce que el parque automotor de vehículos con motor a gasolina y diésel va en aumento, siendo estos últimos los responsables de la emisión de partículas de hollín al ambiente.

El hollín, la ceniza y el polvo son partículas relativamente pesadas que se depositan en el suelo y el aire las limpia rápidamente. Pero cerca de su fuente de origen constituyen con frecuencia un peligro para la salud, ya que pueden causar enfermedades respiratorias crónicas tales como el enfisema y el cáncer [3].

El porcentaje de opacidad es la unidad de medición que determina el grado de opacidad de las emisiones de escape de una fuente móvil a diésel, en el Ecuador esta unidad aporta el Instituto Ecuatoriano de Normalización en la norma técnica NTE INEN 2 202:2000, donde manifiesta que los límites máximos de opacidad de emisiones para fuentes móviles con motor diésel son: A partir del año 2000 en adelante es el $50 \%$ de opacidad y los modelos posteriores al año 2000 es el $60 \%$ de opacidad [4].

Por tal razón la comunidad científica se encuentra en una incansable labor de buscar nuevas fuentes de energía limpia y renovable, que ayude a disminuir las emisiones a la atmósfera y por ende reducir las enfermedades producidas en el ser humano.

Así es como una alternativa al diésel fósil es el biodiésel, el cual es un biocombustible líquido renovable que está compuesto por ésteres monoalquílicos de ácidos grasos de cadena larga derivados de lípidos renovables tales como aceites vegetales que se mezclan, en diferentes porcentajes con gasoil [5], además, es un combustible alternativo de carbón neutro que se debe tomar en cuenta como mecanismo para reducir las emisiones de gases contaminantes que se producen en la combustión [6].
En la actualidad se encuentra varias tecnologías para la producción de biodiésel, los aceites vegetales tienen propiedades que permiten poder manipular su densidad y viscosidad, por lo cual estos aceites son adecuados para la producción de biodiésel [7].

El biodiésel tiene algunas características muy interesantes: No se necesitan grandes cambios en el motor, es biodegradable e inocuo para el ambiente, genera empleo en zonas rurales, diversifica las fuentes de energía $[8]$.

Mediante el uso de biodiésel puro o mezclado, se ha conseguido fantásticos resultados. El uso de este biocombustible ha disminuido considerablemente el potencial de destrucción de la capa de ozono hasta en un $50 \%$, las emisiones de sulfatos y óxidos de azufre son eliminados con el biodiésel puro, estos son componentes principales de la lluvia ácida y se ligan a la formación de partículas principalmente por el contenido de azufre y el contenido de componentes aromáticos pesados [9], el uso del biodiésel en los motores diésel provoca una importante reducción de los hidrocarburos totales sin quemar, del monóxido de carbono y de las partículas en suspensión; las emisiones de los óxidos de nitrógeno son similares o aumentan ligeramente [10].

En lo referente a motores diésel, el biodiésel, dadas las ventajas técnicas, estratégicas y ambientales que ofrece, constituye la mejor alternativa para sustituir parcial o totalmente al combustible diésel derivado del petróleo [11].

$\mathrm{Al}$ utilizar biodiésel se logra disminuir las emisiones de $\mathrm{CO}$ y $\mathrm{CO}_{2}$ presentes en la combustión debido a que una molécula de biodiésel contiene entre 12 y 18 carbones, $\mathrm{y}$, una molécula de diésel puede contener hasta 20 carbones [12].

Las propiedades del biodiésel influyen mucho en las emisiones de hollín al ambiente, por tal motivo la calidad del biodiésel B15 de aceite de higuerilla obtenido son: Su alto índice de cetano 50.5 y su alta viscosidad $5.02 \mathrm{~mm}^{2} / \mathrm{s}$ [11]. De otro lado, el biodiésel de aceite de higuerilla posee puntos de nube y fluidez que le dan ventajas para su uso a bajas temperaturas.

La opacidad de los humos es una medida indirecta del contenido de hollín que tiene el material particulado generado durante la combustión y el escape. El biodiésel en su estructura química aporta oxígeno extra a la combustión, por lo que se espera que facilite la oxidación de las partículas y por tanto se dé una disminución en la opacidad de humos a medida que aumenta su concentración en las mezclas [13].

\subsection{Producción de biodiesel}

El biodiésel se origina debido a una reacción química, como es la transesterificación, donde el glicerol presente en los aceites es suplido por un alcohol, como es el metanol, generalmente empleado en la elaboración con aceites vegetales reciclados, y en mezclas con etanol en aceites nuevos; ante la presencia de un catalizador. 
Se puede utilizar como catalizador, hidróxido de potasio $(\mathrm{KOH})$ o hidróxido de sodio $(\mathrm{NaOH})$, al utilizar $\mathrm{KOH}$, la glicerina resultante del proceso es mucho menos tóxica que al emplear $\mathrm{NaOH}$, además, el hidróxido de potasio se disuelve mucho mejor en metanol. Para que el proceso sea lo más eficiente posible los catalizadores deben tener una pureza de por lo menos $96 \%$ para el $\mathrm{NaOH}$ y alrededor de $92-85 \%$ en el $\mathrm{KOH}$, este último bastante difícil de encontrar con una pureza superior.

La transesterificación consiste en la reacción entre un triglicérido (compuesto por una molécula de glicerol esterificada por tres moléculas de ácidos grasos) contenido en el aceite de ricino y el alcohol (metanol o etanol), originando glicerina y ésteres derivados de los ácidos grasos; al utilizar metanol, el biodiésel estará compuesto por ésteres metílicos. El catalizador se disuelve en alcohol ligero (metanol), para luego ingresar al reactor, junto con el aceite crudo extraído del ricino, donde permanecerán a una temperatura mínima de $45,4{ }^{\circ} \mathrm{C}$ y máxima de $55^{\circ} \mathrm{C}$, a un régimen de $3000 \mathrm{rpm}$, alrededor de 3 horas, dando lugar al proceso de transesterificación, posteriormente se separará la glicerina, la cual pasará por un proceso de lavado, el cual radica en agregar el $26 \%$ de agua en volumen de aceite.

Finalmente, se realiza el proceso de evaporación del agua, a temperaturas de alrededor $100{ }^{\circ} \mathrm{C}$, para luego mezclarlo con diésel fósil en proporción B15 y emplearlo en motores de compresión.

$\mathrm{Al}$ emplear biodiésel se logra reducir las emisiones de monóxido y dióxido de carbono presentes en la combustión, ya que una molécula de biodiésel contiene entre 12 y 18 carbones, y, una molécula de diésel de origen fósil puede contener hasta 20 carbones [14].

\subsection{Propiedades del biodiesel de Higuerilla B15}

Las propiedades fisicoquímicas del biodiésel B15 se determinaron en los laboratorios de petróleos de la Universidad Central obteniendo resultados positivos, en la Tabla 1, se indica los más relevantes:

Tabla 1. Propiedades fisicoquímicas del B15

\begin{tabular}{cc}
\hline Determinación & Resultado \\
\hline Densidad API A 60 F & 544,62 \\
Punto de inflación* ${ }^{\circ} \mathrm{C}$ & 72,1 \\
Azufre* \% P & 0,0148 \\
Índice de cetano calculado & 51 \\
\hline
\end{tabular}

Una de las características principales del combustible diésel es el cetano, este indica la calidad del combustible, como se puede observar se tiene un valor de 51 logrando así en la combustión una mejor expansión y quema de los gases que se generan y por ende una menor contaminación ambiental. Dichos parámetros se encuentran en investigación en proyectos de la carrera de Ingeniería Automotriz de la Universidad Técnica del Norte.

Debido a los grandes beneficios que ha mostrado el uso de biodiésel, la finalidad de este artículo es evidenciar la disminución de la opacidad con el uso de biodiésel B15 a base de higuerilla.

\section{Materiales y métodos}

El presente estudio se basa en un estudio cuantitativo experimental de tipo descriptivo transversal.

Primeramente, de orden cuantitativo en la obtención de resultados de porcentajes de opacidad existentes en cada prueba considerando el tipo de combustible y el régimen del motor, además, en el análisis comparativo de resultados versus las tablas de valores límites de opacidad para motores diésel apegados a la norma INEN 2 207:2002.

De tipo experimental: En la obtención del biodiésel a partir de la planta de higuerilla y en las mediciones de la opacidad efectuados en el sistema de escape del vehículo.

De tipo descriptivo en el análisis de los resultados comparativos, de realización de pruebas y en la descripción en sí del estudio.

De tipo transversal ya que se realizó en un tiempo determinado, permitió obtener la información de forma inmediata y además correlacionar resultados obtenidos desde las diferentes pruebas y los valores que impone la norma nacional vigente y poder determinar los beneficios de uno y otro combustible con el ambiente.

Para poder determinar y analizar la opacidad de los gases de escape para motores diésel se utilizó un opacímetro marca Braen Bee modelo OPA 100 [15] de propiedad de la Universidad Técnica del Norte en la ciudad de Ibarra, calibrado a la normativa vigente ecuatoriana y un banco de pruebas experimentales de motores diésel, para nuestro caso se realizó las pruebas en un motor diésel marca Mazda modelo BT-50 año 2013, 4 cilindros, potencia de 157.30 HP a 3500 RPM y torque de $363 \mathrm{Nm}$ a $1800 \mathrm{RPM}$, sistema de inyección directa electrónica CRD-i recalcando que el motor estuvo en condiciones estándar para las respectivas pruebas.

En cuanto a los combustibles se utilizó biodiésel de higuerilla B15 (15\% de biodiésel y $85 \%$ de diésel fósil) y diésel fósil puro comparándose entre sí los resultados de opacidad obtenidos por cada combustible.

Se realizaron 10 pruebas por cada tipo de combustible y a las RPM anteriormente nombradas previo a una respectiva limpieza del sistema de inyección de combustible con la finalidad de obtener resultados óptimos y más precisos. 
Las mediciones de opacidad se realizaron a la temperatura normal de funcionamiento de $80{ }^{\circ} \mathrm{C}$, una vez cumplida esta condición se procede a calentar el opacímetro siendo una función automática con la que cuenta, seguidamente se realiza una prueba de verificación del opacímetro descartando posibles fugas denominándose a esto como auto cero, finalmente, se introduce la sonda a la abertura del tubo de escape y se realizan las pruebas tanto con biocombustible como con combustible fósil, cada una de ellas realizadas a varios regímenes del motor: 1200, 1500, 2000, 2500, 3000 RPM.

A continuación, en la Tabla 2 se indica los porcentajes de opacidad obtenidas usando diésel fósil, como se indicó anteriormente se realizó a varias RPM tomando en cuenta las condiciones más comunes de funcionamiento del motor donde se puede apreciar que a mayores revoluciones es mayor la opacidad esto se debe al mayor consumo de combustible.

Tabla 2. Pruebas de opacidad diésel fósil

\begin{tabular}{ccccccccc}
\hline \multirow{2}{*}{ RPM } & OPA & OPA & OPA & OPA & OPA & OPA & OPA & PRO. \\
& $\mathbf{1}$ & $\mathbf{2}$ & $\mathbf{3}$ & $\mathbf{4}$ & $\mathbf{5}$ & $\mathbf{6}$ & $\mathbf{7}$ & OPA \\
\hline 1200 & 9,8 & 9 & 10 & 8,7 & 9 & 10 & 9,9 & $\mathbf{9 , 5}$ \\
1500 & 10,9 & 9,7 & 10,8 & 9,7 & 9,7 & 10,1 & 10,7 & $\mathbf{1 0 , 2}$ \\
2000 & 11,7 & 10,8 & 11,1 & 10,8 & 10,5 & 11,8 & 11,1 & $\mathbf{1 1 , 1}$ \\
2500 & 12,9 & 11,3 & 11,9 & 11,5 & 11,6 & 12,3 & 11,9 & $\mathbf{1 1 , 9}$ \\
3000 & 13,5 & 12,8 & 12,6 & 12,7 & 12,5 & 12,7 & 12,6 & $\mathbf{1 2 , 8}$ \\
\hline
\end{tabular}

En la Tabla 3 se observa los resultados de opacidad utilizando biodiésel B15 donde se replicó la misma metodología para realizar dichas pruebas, se obtuvo porcentajes de opacidad más bajos y de la misma manera a mayores revoluciones mayor opacidad.

Tabla 3. Pruebas de opacidad B15

\begin{tabular}{ccccccccc}
\hline \multirow{2}{*}{ RPM } & OPA & OPA & OPA & OPA & OPA & OPA & OPA & PRO. \\
& $\mathbf{1}$ & $\mathbf{2}$ & $\mathbf{3}$ & $\mathbf{4}$ & $\mathbf{5}$ & $\mathbf{6}$ & $\mathbf{7}$ & OPA \\
\hline $\mathbf{1 2 0 0}$ & 0,3 & 0,2 & 0,2 & 0,1 & 0,1 & 0,2 & 0,2 & $\mathbf{0 , 1 9}$ \\
$\mathbf{1 5 0 0}$ & 0,3 & 0,3 & 0,2 & 0,1 & 0,15 & 0,2 & 0,3 & $\mathbf{0 , 2 2}$ \\
$\mathbf{2 0 0 0}$ & 0,4 & 0,4 & 0,4 & 0,1 & 0,2 & 0,2 & 0,4 & $\mathbf{0 , 3}$ \\
$\mathbf{2 5 0 0}$ & 0,6 & 0,7 & 0,8 & 0,1 & 0,2 & 0,3 & 0,6 & $\mathbf{0 , 4 7}$ \\
$\mathbf{3 0 0 0}$ & 0,6 & 1,1 & 1 & 0,55 & 0,5 & 0,5 & 1 & $\mathbf{0 , 7 5}$ \\
\hline
\end{tabular}

De acuerdo con la norma INEN 2 207:2002, la Tabla 4 muestra los límites de opacidad de emisiones para vehículos diésel (pruebas de aceleración libre).

Tabla 4. Porcentaje de opacidad [4]

\begin{tabular}{cc}
\hline Año y modelo & \% opacidad \\
\hline 2000 y posteriores & 50 \\
1999 y anteriores & 60 \\
\hline
\end{tabular}

Las normas internacionales con las que se trabajó para este estudio son las normas EURO donde manifiestan: Vehículos con motor diésel sobrealimentados matriculados por primera vez antes del 01/07/2008:
$30 \%$ de opacidad, tomando en cuenta que esta ley indica la EURO 3 la que se encuentra en vigencia en Ecuador.

\section{Resultados y discusión}

En la Figura 1, se observa el resultado de opacidad utilizando como combustible diésel fósil, dando como resultado un promedio de opacidad de $11,1 \%$, con este resultado el motor aprobaría sin problemas las normas nacionales e internacionales.

Tomando en cuenta que las pruebas se realizan en un motor electrónico y que por sus características de funcionamiento se obtiene niveles bajos de opacidad, se analiza los porcentajes de opacidad a $1200 \mathrm{RPM}$ dando como resultado $9,5 \%$ y a 3000 RPM $12,8 \%$ de opacidad. Dando como resultado un valor promedio de opacidad de $11,1 \%$. Comparando todos los datos se adquiere la ley de opacidad para diésel fósil, en la ecuación (1).

$$
\text { Diésel fósil } l_{o p a}=0.0018(R P M)+7.4619
$$

Donde:

Opa: Opacidad.

RPM: Revoluciones del motor.

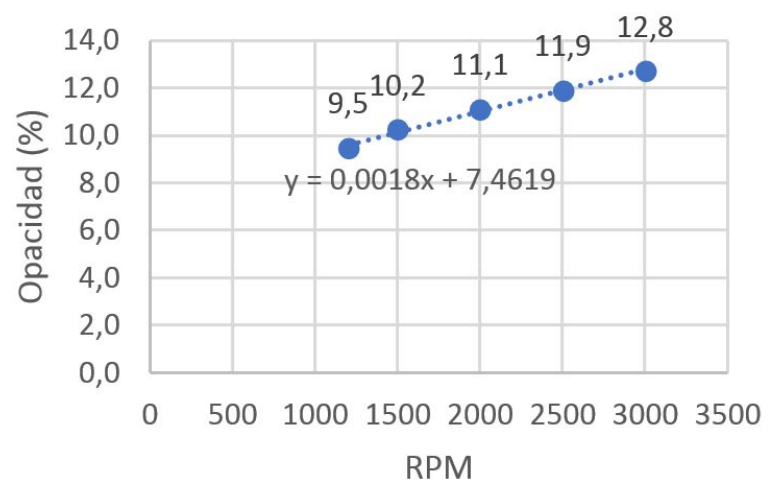

Figura 1. Porcentaje de opacidad diésel fósil

Luego de realizar las pruebas de opacidad utilizando biodiésel B15 en el mismo motor y con las mismas condiciones que las pruebas anteriores, antes de realizar las pruebas se purgó todo el sistema de alimentación para asegurar los resultados obtenidos.

En la Figura 2, se demuestra los resultados de opacidad en 7 pruebas, a diferentes RPM del motor donde a 1200 RPM se encontró una opacidad de $0,19 \%$ y a 3000 RPM se obtuvo $0,75 \%$ de opacidad, obteniendo un valor promedio de opacidad de $0,38 \%$ siendo este resultado óptimo para el motor. De igual manera, se pudo encontrar la ley para calcular la opacidad con el uso de biodiésel B15, que se muestra en la ecuación (2). 


$$
B 15_{\text {opa }}=0.0706 e^{0.0008 R P M}
$$

Donde:

B15: Mezcla biodiésel.

Opa: Opacidad.

RPM: Revoluciones por minuto del motor.

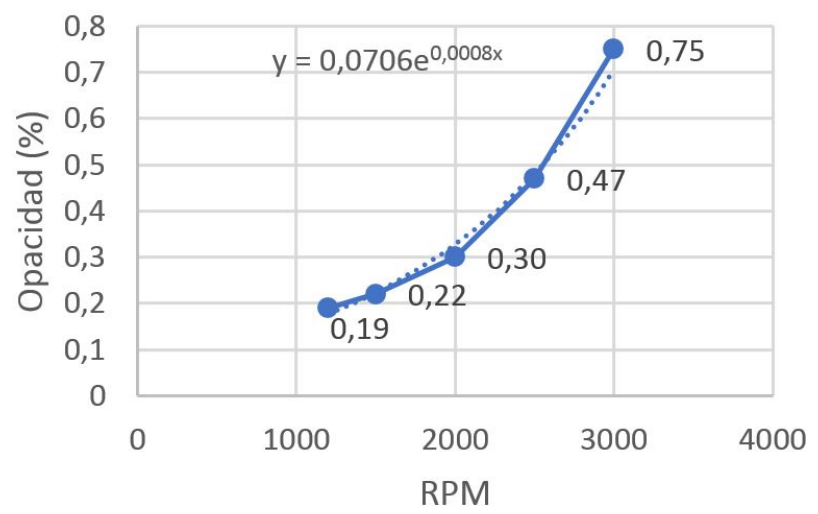

Figura 2. Porcentaje de opacidad B15

La Figura 2 muestra el porcentaje de opacidad de acuerdo con las RPM del motor, se puede apreciar un aumento del porcentaje de opacidad, esto se debe a que a mayores RPM existe mayor consumo de combustible y por ende una mayor cantidad de combustible no quemado originando cantidades superiores de hollín. La norma ecuatoriana INEN 2 207:2007 manifiesta que para los vehículos con motor diésel el límite de opacidad es del $50 \%$ analizando los resultados se concluye que usando biodiésel al $15 \%$ los porcentajes de opacidad disminuyen en valores considerables.

Comparando las gráficas para cada tipo de combustible, B15 y diésel fósil se evidencia una representativa diferencia de valores de opacidad, analizando la línea de tendencia del diésel se observa que según aumenta las RPM del motor es proporcional el porcentaje de opacidad, donde el incremento de opacidad en cada RPM es mayor en comparación al uso de B15 que de la misma manera al aumentar las RPM se eleva el porcentaje de opacidad, pero se puede observar la diferencia en la línea de tendencia, donde en las mismas RPM el aumento de opacidad es menor. Se puede apreciar un aumento promedio de opacidad con diésel fósil de $0,9 \%$ por cada $500 \mathrm{RPM}$ de incremento, mientras que a las mismas condiciones la opacidad con biodiésel B15 aumenta un promedio de 0,17\% disminuyendo un $96 \%$ las emisiones de hollín del motor.

Analizando la normativa nacional y tomando en cuenta las características del motor se puede concluir que el motor aprueba sin faltas el porcentaje de opacidad con los dos combustibles; diésel fósil y biodiésel con mezcla B15, donde respectivamente se tiene el valor de opacidad de $11,1 \%$ y $0,38 \%$, analizando los datos se obtiene una disminución de opacidad del $96 \%$ con el uso de biodiésel B15. Tomando en cuenta las normas internacionales EURO, las mismas que manifiestan que los vehículos no deben sobrepasar un porcentaje de opacidad del $30 \%$, los datos obtenidos aprueban las normas EURO, recalcando que las pruebas se realizaron en un banco de pruebas de motor diésel electrónico Mazda BT-50 en perfectas condiciones tanto sus elementos mecánicos como electrónicos.

En la Figura 3 se muestra los datos comparativos del porcentaje de opacidad con combustible fósil y biocombustible B15.

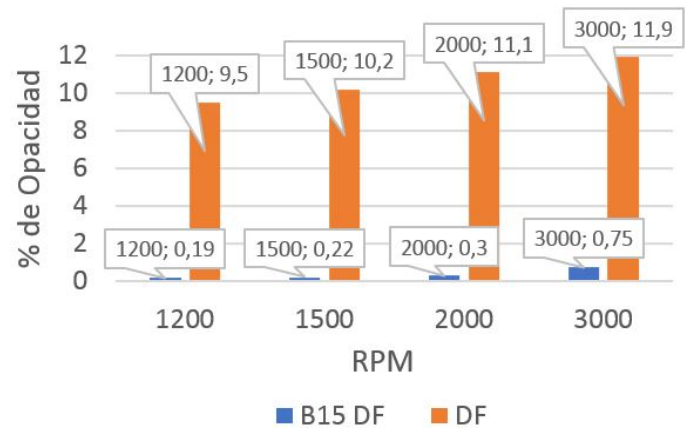

Figura 3. Gráficas comparativas diésel fósil y biodiésel B15

\section{Conclusiones}

Con la utilización de biodiésel B15 a base de aceite de higuerilla la opacidad disminuye un $96 \%$ con respecto a la utilización del diésel fósil, esto se debe al aumento de la temperatura en la cámara de combustión y a la mejor oxidación de la mezcla.

Con las ecuaciones obtenidas en este estudio se puede conocer de una manera más rápida la opacidad con el uso de los dos combustibles, considerando el uso de motores de las mismas características a la del estudio.

Usando biodiésel se puede mitigar en un gran porcentaje las enfermedades cancerígenas en los pulmones de las personas, así es como manifiesta la OMS donde las partículas más pequeñas de hollín son las causantes de este problema.

La maqueta de motor utilizada para el estudio se encuentra en óptimas condiciones, por tal motivo sus valores con diésel fósil y biodiésel son bajos, en vehículos de calle estos porcentajes pueden variar en pequeños porcentajes.

\section{Referencias}

[1] J. Hernández Gaitán, G. Zambrano Ruano, and C. Rossi, "Emisión de gases en vehículos experimentales diesel-biodiesel," Revista de la Universidad del Valle de guatemala, no. 27, pp. 64-67, 2014. [Online]. Available: https://goo.gl/AfA1vR 
[2] A. A. Agudelo, J. Agudelo, and P. Benjumea, "Diagnóstico exergético del proceso de combustión en un motor diésel," Revista Facultad de Ingeniería Universidad de Antioquia, no. 45, pp. 41-53, 09 2008. [Online]. Available: https://goo.gl/AbLZ2z

[3] Z. Z. Yu and L. Jing, "Effects of exhaust post-treatment technology on diesel engine emissions," in Proceedings 2011 International Conference on Transportation, $\mathrm{Me}$ chanical, and Electrical Engineering (TMEE), Dec 2011, pp. 1804-1807. [Online]. Available: https://doi.org/10.1109/TMEE.2011.6199564

[4] INEN, Gestión ambiental. Aire. Vehículos automotores. determinación de la opacidad de emisiones de escape de motores de diésel mediante la prueba estática. Método de aceleración libre, Instituto Ecuatoriano de Normalización Std., 2010. [Online]. Available: https://goo.gl/211cW7

[5] F. Marcos Martín, J. Almazán Gárate, and C. Palomino Monzón, "Biodiésel de algas," Energética XXI, no. 74, pp. 100-104, 2008. [Online]. Available: https://goo.gl/UFhkYS

[6] S. Imtenan, M. Varman, H. Masjuki, M. Kalam, H. Sajjad, M. Arbab, and I. R. Fattah, "Impact of low temperature combustion attaining strategies on diesel engine emissions for diesel and biodiesels: A review," Energy Conversion and Management, vol. 80, Supplement C, pp. 329-356, 2014. [Online]. Available: https://doi.org/10.1016/j.enconman.2014.01.020

[7] C. S. Aalam, C. Saravanan, and M. Kannan, "Experimental investigations on a crdi system assisted diesel engine fuelled with aluminium oxide nanoparticles blended biodiesel," Alexandria Engineering Journal, vol. 54, no. 3, pp. 351-358, 2015. [Online]. Available: https://doi.org/10.1016/j.aej.2015.04.009

[8] E. Jiaqiang, P. Minhhieu, D. Zhao, D. Yuanwang, L. DucHieu, Z. Wei, Z. Hao, L. Teng, P. Qingguo, and Z. Zhiqing, "Effect of different technologies on combustion and emissions of the diesel engine fueled with biodiesel: A review," Renewable and Sustainable Energy Reviews, vol. 80, Supplement C, pp. 620-647, 2017. [Online]. Available: https://doi.org/10.1016/j.rser.2017.05.250

[9] J. Azjargal, "The comparison of combustion characteristics and performances of diesel engine fueled with biodiesel and diesel blends," in 2014 9th International Forum on Strategic Technology (IFOST), Oct 2014, pp. 313-317. [Online]. Available: https://doi.org/10.1109/IFOST.2014.6991129

[10] E. Bernard. (2010) Biodiésel: Los aspectos mecánicos en los vehículos. Centro Nacional de la Producción mss Limpia, Costa Rica. [Online]. Available: https://goo.gl/aRbzGf

[11] A. Benavides, P. Benjumea, and V. Pashova, "El biodiésel de aceite de higuerilla como combustible alternativo para motores diésel," $D Y N A$, vol. 74, no. 153, pp. 141-150, 2007. [Online]. Available: https://goo.gl/FrAArX

[12] J. M. Amaris, D. A. Manrique, and J. E. Jaramillo, "Biocombustibles líquidos en Colombia y su impacto en motores de combustión interna," Fuentes, el reventón energético, vol. 13, no. 2, pp. 23-34, 2015, hybrid evolutionary systems for manufacturing processes. [Online]. Available: https://doi.org/10.18273/revfue.v13n2-2015003

[13] J. Agudelo, P. Benjumea, and J. Pérez, "Pruebas cortas en ruta en un vehículo tipo microbús con biodiésel de aceite de palma colombiano," Scientia et Technica, vol. 1, no. 24, pp. 163-168, 2004. [Online]. Available: http://dx.doi.org/10.22517/23447214.7321

[14] T. Saba, J. Estephane, B. E. Khoury, M. E. Khoury, M. Khazma, H. E. Zakhem, and S. Aouad, "Biodiesel production from refined sunflower vegetable oil over koh/zsm5 catalysts," Renewable Energy, vol. 90, Supplement C, pp. 301-306, 2016. [Online]. Available: https://doi.org/10.1016/j.renene.2016.01.009

[15] Globaltech. (2016) Opacímetro brain bee opa-100. [Online]. Available: https://goo.gl/QPiHA2 


\section{Normas PARA PUBLICAR EN LA REVISTA INGENIUS}

\section{Información General}

INGENIUS es una publicación científica de la Universidad Politécnica Salesiana de Ecuador, editada desde enero de 2007, con periodicidad fija semestral, especializada en Ingeniería Mecánica, Ingeniería Eléctrica, Electrónica, Ciencias de la Computación y su integración en lo que actualmente se conoce como Mecatrónica; estas líneas de acción fortalecen áreas como automatización, control, robótica entre otras.

Es una revista científica arbitrada, que utiliza el sistema de evaluación externa por expertos (peer-review), bajo metodología de pares ciegos (doble-blind review), conforme a las normas de publicación del Institute of Electrical and Electronics Engineers (IEEE). El cumplimiento de este sistema permite garantizar a los autores un proceso de revisión objetivo, imparcial y transparente, lo que facilita a la publicación su inclusión en bases de datos, repositorios e indexaciones internacionales de referencia.

INGENIUS se encuentra indexada en el directorio y catálogo selectivo del Sistema Regional de Información en Línea para Revistas Científicas de América Latina, el Caribe, España y Portugal - Latindex, en el Directorio de Revistas de Acceso Abierto - DOAJ, en la Matriz de Información para el Análisis de Revistas MIAR, en la Red Iberoamericana de Innovación y Conocimiento Científico - REDIB y en repositorios, bibliotecas y catálogos especializados de Iberoamérica.

La revista se edita en doble versión: impresa (ISSN: 1390-650X) y electrónica (e-ISSN: 1390-860X), en idioma español, siendo identificado además cada trabajo con un DOI (Digital
Object Identifier System). Los artículos enviados a la revista INGENIUS deben ajustarse a los siguientes criterios:

\section{Alcance y Política}

\subsection{Temática}

Contribuciones originales en materia de Ingeniería Mecánica, Ingeniería Eléctrica y Electrónica, Ciencias de la computación y su integración en lo que actualmente se conoce como Mecatrónica, así como áreas afines: Automatización, Control, Domótica, Robótica en sus diferentes ámbitos de acción y todas aquellas disciplinas conexas interdisciplinarmente con la línea temática central.

Podrán ser publicados todos los trabajos realizados por investigadores nacionales o extranjeros, una vez que cumplan los criterios de calidad científica requeridos.

\subsection{Aportaciones}

La revista INGENIUS publica preferentemente artículos relacionados con investigaciones empíricas, siendo también admisibles informes de desarrollo tecnológico, propuestas de modelos e innovaciones, productos de la elaboración de tesis de grado y posgrado siempre que sean un aporte para el campo de la ciencia y tecnología, así como selectas revisiones del estado del arte (state-of-the-art).

- Investigaciones: 5.000 a 6.500 palabras de texto, incluyendo título, resúmenes, palabras clave, tablas y referencias.

- Informes, propuestas y productos: 5.000 a 6.500 palabras de texto, incluyendo título, resúmenes, tablas y referencias. 
- Revisiones: 6.000 a 7.000 palabras de texto, incluidas tablas y referencias. Se valora especialmente las referencias justificadas, actuales y selectivas de alrededor de unas 40 obras.

La revista INGENIUS publica trabajos originales e inéditos redactados en español e inglés, no pueden haber sido publicados a través de ningún medio impreso ni electrónico, ni estar en proceso de arbitraje o publicación.

Todo artículo será sometido a un riguroso proceso de arbitraje; la evaluación del artículo se hará conforme a criterios de originalidad, pertinencia, actualidad, aportes, rigurosidad científica y cumplimiento de las normas editoriales establecidas.

Por tratarse de una publicación arbitrada, el Consejo Editorial aprueba su publicación en base al concepto de pares especializados. La recepción de un documento no implica compromiso de publicación.

Es indispensable presentar una carta de presentación y cover letter que se puede descargar de: <https://goo.gl/xB0wEl>.

Las contribuciones deben ser enviadas única y exclusivamente a través del OJS (Open Journal System) $<$ https://goo.gl/4xxjuo $>$, en el cual todos los autores deben registrarse como usuario previamente. Para cualquier consulta del procedimiento se debe contactar a:

$<$ revistaingenius@ups.edu.ec>,

$<$ jcalle@ups.edu.ec>ó

$<$ mquinde@ups.edu.ec $>$.

\section{Presentación y estructura de los manuscritos}

Para aquellos trabajos que se traten de investigaciones de carácter empírico, los manuscritos seguirán la estructura IMRDC (Introducción, Materiales y Métodos, Resultados y Discusión y Conclusiones), siendo opcionales los epígrafes de Notas y Apoyos. Aquellos tra- bajos que por el contrario se traten de informes, estudios, propuestas y revisiones podrán ser más flexibles en sus epígrafes, especialmente en Materiales y métodos, Resultados, y Discusión y Conclusiones. En todas las tipologías de trabajos son obligatorias las Referencias.

Los artículos pueden estar escritos sobre Microsoft Word (.doc o .docx) o $\operatorname{ATEX}$ (.tex). La plantilla a ser utilizada puede ser descargada del sitio web de la revista, en formato de Microsoft Word en: <https://goo.gl/ZA2XAk $>$, mientras que para $\mathrm{AT}_{\mathrm{E} X} \mathrm{X}$ : $<$ https://goo gl/Mwv8IC > , es necesario que el archivo esté anonimizado en Propiedades de Archivo, de forma que no aparezca la identificación de autor/es.

Las Figuras, Gráficos y/o Ilustraciones, así como las Tablas deberán estar numeradas secuencialmente incluyendo una descripción explicativa para cada una. Las ecuaciones incluidas en el artículo deberán también estar numeradas; tanto las figuras, tablas y ecuaciones deben estar citadas en el texto.

Use espacio después de punto, comas y signos de interrogación.

Use enter al final de cada párrafo, título encabezamiento. No use enter en ningún otro lugar, deje al programa procesador de palabras romper automáticamente las líneas.

No centre encabezamientos o subencabezamientos ya que deben estar alineados a la izquierda.

Las Tablas deben estar creadas en el mismo programa usado para el cuerpo del documento. Use tabuladores, no espacios, para crear columnas. Recuerde que el tamaño final de las páginas impresas será de 21 x $28 \mathrm{~cm}$, por lo tanto las tablas deben estar diseñadas para ajustarse al espacio de la impresión final. 


\subsection{Estructura de los manuscritos}

\subsubsection{Presentación y Cover Letter}

1. Título (español) / Title (inglés): Conciso pero informativo, en castellano en primera línea y en inglés en segunda, cuando el artículo sea escrito en español y viceversa si está escrito en inglés.

2. Autores y adscripción: Nombre y Apellidos completo de cada autor, organizados por orden de prelación y su adscripción institucional con referencia al final de la primera hoja, donde tiene que incluir: Dependencia a la que pertenece, Institución a la que pertenece, país, ORCID. Se aceptarán como máximo 5 autores, aunque pudieran existir excepciones justificadas por la complejidad y extensión del tema.

3. Resumen (español) / Abstract (inglés): Tendrá como extensión máxima 230 palabras, en español y en inglés. En el resumen se describirá de forma concisa y en este orden: 1) Justificación del tema; 2) Objetivos; 3) Metodología y muestra; 4) Principales resultados; 5) Principales conclusiones.

4. Palabras clave (español) / Keywords (inglés): Se deben exponer 6 palabras clave por cada versión idiomática relacionados directamente con el tema del trabajo. Será valorado positivamente el uso de las palabras claves expuestas en el Thesaurus de la UNESCO.

5. Presentación (Cover Letter): Una declaración de que el manuscrito se trata de una aportación original, no enviada ni en proceso de evaluación en otra revista, con la confirmación de las autorías firmantes, aceptación (si procede) de cambios formales en el manuscrito conforme a las normas y cesión parcial de derechos a la editorial, según el formato establecido en: $<$ https://goo.gl/XAc9a3 $>$.

\subsubsection{Manuscrito}

1. Título (español) / Title (inglés): Conciso pero informativo, en castellano en primera línea y en inglés en segunda, cuando el artículo sea escrito en español y viceversa si está escrito en inglés.

2. Autores y adscripción: Nombre y Apellidos completo de cada autor, organizados por orden de prelación y su adscripción institucional con referencia al final de la primera hoja, donde tiene que incluir: Dependencia a la que pertenece, Institución a la que pertenece, país, ORCID. Se aceptarán como máximo 5 autores, aunque pudieran existir excepciones justificadas por la complejidad y extensión del tema.

3. Resumen (español) / Abstract (inglés): Tendrá como extensión máxima 230 palabras, en español y en inglés. En el resumen se describirá de forma concisa y en este orden: 1) Justificación del tema; 2) Objetivos; 3) Metodología y muestra; 4) Principales resultados; 5) Principales conclusiones.

4. Palabras clave (español) / Keywords (inglés): Se deben exponer 6 palabras clave por cada versión idiomática relacionados directamente con el tema del trabajo. Será valorado positivamente el uso de las palabras claves expuestas en el Thesaurus de la UNESCO.

5. Introducción: Debe incluir el planteamiento del problema, el contexto de la problemática, la justificación, fundamentos y propósito del estudio, utilizando citas bibliográficas, así como la literatura más significativa y actual del tema a escala nacional e internacional. 
6. Materiales y métodos: Debe ser redactado de forma que el lector pueda comprender con facilidad el desarrollo de la investigación. En su caso, describirá la metodología, la muestra y la forma de muestreo, así como se hará referencia al tipo de análisis estadístico empleado. Si se trata de una metodología original, es necesario exponer las razones que han conducido a su empleo y describir sus posibles limitaciones.

7. Análisis y resultados: Se procurará resaltar las observaciones más importantes, describiéndose, sin hacer juicios de valor, el material y métodos empleados. Deberán aparecer en una secuencia lógica en el texto y las tablas y figuras imprescindibles evitando la duplicidad de datos.

8. Discusión y Conclusiones: Resumirá los hallazgos más importantes, relacionando las propias observaciones con estudios de interés, señalando aportaciones y limitaciones, sin redundar datos ya comentados en otros apartados. Asimismo, debe incluir las deducciones y líneas para futuras investigaciones.

9. Apoyos y agradecimientos (opcionales): El Council Science Editors recomienda al autor/es especificar la fuente de financiación de la investigación. Se considerarán prioritarios los trabajos con aval de proyectos competitivos nacionales e internacionales.

10. Las notas (opcionales): Se deberán incluir solo en caso necesario, al final del artículo (antes de las referencias). Deben anotarse manualmente, ya que el sistema de notas al pie o al final de Word no es reconocido por los sistemas de maquetación. Los números de notas se colocan en superíndice, tanto en el texto como en la nota final. No se permiten notas que recojan citas bibliográficas simples ( $\sin$ comentarios), pues éstas deben ir en las referencias.

11. Referencias Bibliográficas: Las citas bibliográficas deben reseñarse en forma de referencias al texto. Bajo ningún caso deben incluirse referencias no citadas en el texto. Su número debe ser suficiente para contextualizar el marco teórico con criterios de actualidad e importancia. Se presentarán secuencialmente en orden de aparición, según corresponda siguiendo el formato de la IEEE.

\subsection{Normas para las referencias Bibliográficas}

Artículos de revistas:

[1] J. Riess, J. J. Abbas, "Adaptive control of cyclic movements as muscles fatigue using functional neuromuscular stimulation". IEEE Trans. Neural Syst. Rehabil. Eng vol. 9, pp.326-330, 2001. [Onine]. Available: https://doi.org/10.1109/7333.948462 Libros:

[1] G. O. Young, "Synthetic structure of industrial plastics" in Plastics, 2nd ed., vol. 3, J. Peters, Ed. New York: McGraw-Hill, 1964, pp. 15-64.

Reportes Técnicos:

[1] M. A. Brusberg and E. N. Clark, "Installation, operation, and data evaluation of an oblique-incidence ionosphere sounder system," in Radio Propagation Characteristics of the Washington-Honolulu Path, Stanford Res. Inst., Stanford, CA, Contract NOBSR-87615, Final Rep., Feb. 1995, vol. 1

Artículos presentados en conferencias (No publicados):

[1] Vázquez, Rolando, Presentación curso "Realidad Virtual". National Instruments. Colombia, 2009. 
Articulos de Memorias de Conferencias (Publicados):

[1] L. I. Ruiz, A. García, J. García, G. Taboada. "Criterios para la optimización de sistemas eléctricos en refinerías de la industria petrolera: influencia y análisis en el equipo eléctrico," IEEE CONCAPAN XXVIII, Guatemala 2008.

Tesis:

[1] L.M. Moreno, "Computación paralela y entornos heterogéneos," Tesis doctoral, Dep. Estadística, Investigación Operativa y Computación, Universidad de La Laguna, La Laguna, 2005.

\section{Estándares:}

[1] IEEE Guide for Application of Power Apparatus Bushings, IEEE Standard C57.19.100-1995, Aug. 1995.

Patentes:

[1] J. P. Wilkinson, "Nonlinear resonant circuit devices, U.S. Patent 3624 125, July 16, 1990.

\section{Manuales:}

[1] Motorola Semiconductor Data Manual, Motorola Semiconductor Products Inc., Phoenix, AZ, 1989.

Recursos de internet:

[1] E. H. Miller, "A note on reflector arrays" [Online]. Available: https://goo.gl/4cJkCF

\subsection{Epígrafes, tablas y figuras}

Los epígrafes del cuerpo del artículo se deben numerar en arábigo. Irán sin caja completa de mayúsculas, ni subrayados, ni negritas. La numeración ha de ser como máximo de tres niveles: 1. / 1.1. / 1.1.1. Al final de cada epígrafe numerado se dará un enter para continuar con el párrafo correspondiente.

Las tablas deben presentarse incluidas en el texto según orden de aparición, numeradas en arábigo y subtituladas con la descripción del contenido, el subtítulo debe ir en la parte superior de la tabla justificado a la izquierda.

Las figuras pueden ser dibujos lineales, mapas o fotografías de medios tonos en blanco y negro o a color en resolución de 300 dpi. No combine fotografías y dibujos lineales en la misma figura.

Diseñe las figuras para que se ajusten eventualmente al tamaño final de la revista 21 x 28 $\mathrm{cm}$. Asegúrese de que las inscripciones o detalles, así como las líneas, tengan tamaños y grosores adecuados de tal manera que no queden ilegibles cuando sean reducidos a su tamaño final (números, letras y símbolos deben ser reducidos al menos a 2,5 mm de altura después que las ilustraciones han sido reducidas para ajustarse a la página impresa). Idealmente, las ilustraciones lineales deben ser preparadas a aproximadamente un cuarto de su tamaño final de publicación. Diferentes elementos en la misma figura deben ser deletreados a, b, c, etc.

Las fotografías deben grabarse con alto contraste y en alta resolución. Recuerde que las fotografías frecuentemente pierden contraste en el proceso de impresión.

Dibujos lineales y mapas deben ser preparados en color negro.

El texto de las figuras y mapas debe escribirse con letras fácilmente legibles.

Si las figuras han sido previamente usadas, es de responsabilidad del autor obtener el permiso correspondiente para evitar problemas posteriores relacionados con los derechos de autor.

Cada figura debe ser entregada en un archivo aparte, ya sea como mapa de bits (.jpg, .bmp, .gif, o .png) o como gráfico vectorial (.ps, .eps, .pdf).

\section{Proceso de envío}

El envío será a través de la plataforma OJS de la revista, <https://goo.gl/4xxjuo>, de 
manera obligatoria dos archivos se deberán cargar, como archivo original el manuscrito en .pdf sin datos de autor y anonimizado, mientras que en archivos complementarios se deberá cargar la presentación y cover letter de acuerdo a lo antes descrito, adicionalmente se deberá cargar el manuscrito completo en .doc o .docx (archivo de Word), es decir con los datos del autor/es y su adscripción institucional; también se deben cargar en archivos independientes las figuras numeradas de acuerdo a lo correspondiente en el manuscrito (como mapa de bits .jpg, .bmp, .gif, o .png o como gráfico vectorial .ps, .eps, .pdf), en calidad como mínima de 300 dpi.

Todos los autores deben ingresar la información requerida en la plataforma OJS y uno solo de los autores será el responsable de correspondencia.

Una vez enviada la contribución el sistema automáticamente enviará al autor para correspondencia un mail de confirmación de recepción de la contribución.

\section{Proceso Editorial}

Una vez que el manuscrito ha sido receptado en OJS se realiza una primera comprobación por parte del equipo editorial de los siguientes puntos:

- La temática se encuentre en concordancia con los criterios de la revista.

- Debe tener la estructura IMRDC

- Debe estar en el formato de la revista INGENIUS.

- Debe utilizar la forma de citación de la IEEE.

- Todas las referencias bibliográficas deben estar citadas en el texto del manuscrito al igual que las tablas, figuras y ecuaciones.

- El manuscrito es original, para esto se utiliza un software para determinar plagio.
La comprobación antes descrita puede llevar hasta 4 semanas.

Si alguno de los puntos anteriores no está completo o existe inconsistencia, se enviará un correo al autor para que realize las correcciones solicitadas. El autor ejecutará las correcciones y enviará nuevamente la contribución a través de un mail como respuesta a la notificación y también cargará el manuscrito corregido en archivos complementarios del OJS.

El equipo editorial verificará que las correcciones solicitadas han sido incorporadas, si cumple, el manuscrito iniciará la segunda parte del proceso y podrá ser seguido por el autor a través de OJS, caso contrario se notificará al autor y se archivará el manuscrito.

La segunda fase del proceso consiste en la evaluación bajo la metodología de pares ciegos (doble-blind review), en los que se incluyen expertos nacionales y extranjeros considerando los siguientes pasos:

- El editor asigna dos o más revisores para el artículo.

- Una vez revisado el artículo, los revisores remitirán el informe de la evaluación, con uno de los siguientes resultados.

- Publicable

- Publicable con cambios sugeridos

- Publicable con cambios obligatorios

- No Publicable

- El editor una vez recibida la evaluación por parte de los revisores analizará los resultados y determinará si el artículo es aceptado o negado.

- Si el artículo es aceptado se notificará al autor para que realice las correcciones en caso de ser requeridas y se continuará con el proceso editorial correspondiente. 
- Si el artículo es negado se notificará al autor y se archivará el manuscrito.

- En los dos casos anteriores se enviará el resultado de la evaluación de los revisores y sus respectivas recomendaciones.

La segunda fase del proceso dura 4 semanas como mínimo, luego de transcurridas las mismas se notificará al autor dando instrucciones para continuar con el proceso.

\section{Publicación}

La revista INGENIUS publica dos números por año, el 1 de enero y el 1 de julio por lo tanto es importante considerar las fechas para el envío de los artículos y su correspondiente publicación. Los artículos recibidos hasta el mes de octubre serán considerados para la publicación de enero y aquellos que se reciban hasta abril para la publicación de julio. 


\section{GUIDELINES FOR PUBLICATION IN INGENIUS JOURNAL}

\section{General Information}

INGENIUS is a scientific publication of the Universidad Politécnica Salesiana of Ecuador, published since January 2007, with a fixed biannual periodicity, specialized in Mechanical Engineering, Electrical Engineering, Electronics, Computer Science and its integration in what is now known as Mechatronics; these lines of action strengthen areas such as automation, control, robotics, among others..

It is a scientific journal, which uses the peerreview system, under double-blind review methodology, according to the publication standards of the Institute of Electrical and Electronics Engineers (IEEE). Compliance with this system allows authors to guarantee an objective, impartial and transparent review process, which facilitates the publication of their inclusion in reference databases, repositories and international indexing.

INGENIUS is indexed in the directory and selective catalog of the Regional Online Information System for Scientific Journals of Latin America, the Caribbean, Spain and Portugal (Latindex), in the Directory of Journals of Open Access DOAJ, In the Information Matrix for the Analysis of Journals, MIAR, In the Ibero-American Network of Innovation and Scientific Knowledge, REDIB and in repositories, libraries and specialized catalogs of Latin America.

The journal is published in a double version: printed (ISSN: 1390-650X) and digital (e-ISSN: 1390-860X), in Spanish, each work being identified with a DOI (Digital Object Identifier System. The articles sent to InGEnius magazine must comply with the following criteria:

\section{Scope and policy}

\subsection{Theme}

Original contributions in Mechanical Engineering, Electrical and Electronic Engineering, Computer Science and its integration in what is now known as Mechatronics, as well as related areas: Automation, Control, Domotics, Robotics in their different fields of action and all those related disciplines with the same central theme.

All the work carried out by national or foreign researchers may be published once they meet the required scientific quality criteria.

\subsection{Contributions}

INGENIUS Journal preferably publishes articles related to empirical research, and also reports of technological development, proposals for models and innovations, products for the elaboration of graduate and postgraduate thesis that contribute to the field of science and technology, as well as select revisions of literature. (state-of-the-art).

- Research: 5,000 to 6,500 words of text, including title, abstracts, descriptors, charts and references.

- Reports: 5,000 to 6,500 words of text, including title, abstracts, charts and references.

- Reviews: 6,000 to 7,000 words of text, including charts and references. Current, selective and justified references, would be specially valued from among 40 works The Ingenius Journal publishes original and unpublished works written in Spanish and English, they may not have been published 
through any printed or electronic media, nor be in the process of arbitration or publication.

Every article will be subjected to a rigorous arbitration process; the evaluation of the article will be made according to criteria of originality, relevance, relevance, contributions, scientific rigor and compliance with established editorial guidelines.

Being an arbitrated publication, the Editorial Board approves its publication based on the concept of specialized pairs. The reception of a document does not imply commitment of publication.

It is essential to present a letter of presentation and grant of rights which can be downloaded from: < urlhttps://goo.gl/ZNkMRD $>$.

Contributions must be exclusively sent and through the OJS (Open Journal Sys-tem) $<$ https://goo.gl/JF7dWT $>$. In which all authors must previously register as a user. For any consultation of the procedure you should contact:

$<$ revistaingenius@ups.edu.ec $>$,

$<$ jcalle@ups.edu.ec $>$ ó

$<$ mquinde@ups.edu.ec $>$.

\section{Presentation and structure of the ma- nuscripts}

For those works that are empirical investigations, the manuscripts will follow the IMRDC structure (Introduction, Materials and Methods, Results and Discussion and Conclusions), being optional the Notes and Supports. Those papers that, on the contrary, deal with reports, studies, proposals and reviews may be more flexible in their epigraphs, particularly in material and methods, analysis, results, discussion and conclusions. In all typologies of works, references are mandatory.

Articles may be written on Microsoft Word (.doc or .docx) or $\operatorname{AT}_{\mathrm{EX}}$ (.tex). The template to be used can be downloaded from the journal's website, a, <https://goo.gl/gtCg6m>, while for $\mathrm{HT}_{\mathrm{E} X}$ in <https://goo.gl/hrHzzQ>, it is necessary that the file be anonymised in Properties of File, so that the author(s) ID is not displayed.

Figures, Graphs and/or Illustrations, as well as Charts shall be numbered sequentially including an explanatory description for each. The equations included in the article must also be numbered; the figures, charts and equations must be cited in the text.

Use space after point, commas and question marks.

Use "enter" at the end of each paragraph and title heading. Do not use ${ }^{\text {en }}$ ter. ${ }^{a}$ nywhere else, let the word processor program automatically break the lines.

Do not center headings or subheadings as they should be aligned to the left.

Charts must be created in the same program used for the document body, but must be stored in a separate file. Use tabs, not spaces, to create columns. Remember that the final size of printed pages will be $21 \times 28 \mathrm{~cm}$, so the tables must be designed to fit the final print space.

\subsection{Structure of the manuscripts}

\subsubsection{Presentation and cover letter}

1. Título (español) / Title (inglés): Concise but informative, in Spanish on the front line and in English on the second, when the article is written in Spanish and vice versa if it is written in English.

2. Authors and affiliations: Full name and surname of each author, organized by order of priority and their institutional affiliation with reference to the end of the first sheet, where it must include: 
Dependency to which belongs within the institution, Institution to which he/she belongs, country, ORCID. A maximum of 5 authors will be accepted, although there may be exceptions justified by the complexity and extent of the topic.

3. Abstract (Spanish) / Abstract (English): It will have a maximum extension of 230 words, first in Spanish and then in English. : 1) Justification of the topic; 2) Objectives; 3) Methodology and sample; 4) Main results; 5) Main conclusions.

4. Keywords (Spanish) / Keywords (English): 6 descriptors must be presented for each language version directly related to the subject of the work. The use of the key words set out in UNESCO's Thesaurus will be positively valued.

5. Presentation (Cover Letter): A statement that the manuscript is an original contribution, not submission or evaluation process in another journal, with the confirmation of the signatory authors, acceptance (if applicable) of formal changes in the manuscript according to the guidelines and partial assignment of rights to the publisher, according to the format established in: <https://goo.gl/ZNkMRD>

\subsubsection{Manuscript}

1. Título (español) / Title (inglés): Concise but informative, in Spanish on the front line and in English on the second, when the article is written in Spanish and vice versa if it is written in English.

2. Authors and affiliations: Full name and surname of each author, organized by order of priority and their institutional affiliation with reference to the end of the first sheet, where it must include: Dependency to which belongs within the institution, Institution to which he/she belongs, country, ORCID. A maximum of 5 authors will be accepted, although there may be exceptions justified by the complexity and extent of the topic.

3. Abstract (Spanish) / Abstract (English): It will have a maximum extension of 230 words, first in Spanish and then in English. : 1) Justification of the topic; 2) Objectives; 3) Methodology and sample; 4) Main results; 5) Main conclusions.

\section{Keywords (Spanish) / Keywords} (English): 6 descriptors must be presented for each language version directly related to the subject of the work. The use of the key words set out in UNESCO's Thesaurus will be positively valued.

5. Introduction: It should include the problem statement, context of the problem, justification, rationale and purpose of the study, using bibliographical citations, as well as the most significant and current literature on the topic at national and international level.

6. Material and methods: It must be written so that the reader can easily understand the development of the research. If applicable, it will describe the methodology, the sample and the form of sampling, as well as the type of statistical analysis used. If it is an original methodology, it is necessary to explain the reasons that led to its use and to describe its possible limitations.

7. Analysis and results: It will try to highlight the most important observations, describing, without making value judgments, the material and methods used. They will appear in a logical sequence in the text and the essential charts and figures avoiding the duplication of data.

8. Discussion and Conclusions: It will summarize the most important findings, 
relating the observations themselves to relevant studies, indicating contributions and limitations, without adding data already mentioned in other sections. It should also include deductions and lines for future research.

9. Supports and acknowledgments (optional): The Council Science Editors recommends the author (s) to specify the source of funding for the research. Priority will be given to projects supported by national and international competitive projects.

10. The notes (optional): will go, only if necessary, at the end of the article (before the references). They must be manually annotated, since the system of footnotes or the end of Word is not recognized by the layout systems. The numbers of notes are placed in superscript, both in the text and in the final note. The numbers of notes are placed in superscript, both in the text and in the final note. No notes are allowed that collect simple bibliographic citations (without comments), as these should go in the references.

11. References: Bibliographical citations should be reviewed in the form of references to the text. Under no circumstances should references mentioned in the text not be included. Their number should be sufficient to contextualize the theoretical framework with current and important criteria. They will be presented sequentially in order of appearance, as appropriate following the format of the IEEE.

\subsection{Guidelines for Bibliographical references}

Journal articles:

[1] J. Riess, J. J. Abbas, "Adaptive control of cyclic movements as muscles fatigue using functional neuromuscular stimulation". IEEE Trans. Neural Syst. Rehabil. Eng vol. 9, pp.326-330, 2001. [Onine]. Available: https://doi.org/10.1109/7333.948462 Books:

[1] G. O. Young, "Synthetic structure of industrial plastics" in Plastics, 2nd ed., vol. 3, J. Peters, Ed. New York: McGraw-Hill, 1964, pp. 15-64.

\section{Technical reports:}

[1] M. A. Brusberg and E. N. Clark, "Installation, operation, and data evaluation of an oblique-incidence ionosphere sounder system," in Radio Propagation Characteristics of the Washington-Honolulu Path, Stanford Res. Inst., Stanford, CA, Contract NOBSR-87615, Final Rep., Feb. 1995, vol. 1

Articles presented in confeences (unpubished):

[1] Vázquez, Rolando, Presentación curso "Realidad Virtual". National Instruments. Colombia, 2009.

Articles of memories of Conferences (Published):

[1] L. I. Ruiz, A. García, J. García, G. Taboada. "Criterios para la optimización de sistemas eléctricos en refinerías de la industria petrolera: influencia y análisis en el equipo eléctrico," IEEE CONCAPAN XXVIII, Guatemala 2008.

Thesis:

[1] L.M. Moreno, "Computación paralela y entornos heterogéneos," Tesis doctoral, Dep. Estadística, Investigación Operativa y Computación, Universidad de La Laguna, La Laguna, 2005.

\section{Guidelines:}

[1] IEEE Guide for Application of Power Apparatus Bushings, IEEE Standard C57.19.100-1995, Aug. 1995.

\section{Patents:}

[1] J. P. Wilkinson, "Nonlinear resonant cir- 
cuit devices, U.S. Patent 3624 125, July $16,1990$.

Manuals:

[1] Motorola Semiconductor Data Manual, Motorola Semiconductor Products Inc., Phoenix, AZ, 1989.

Internet resources:

[1] E. H. Miller, "A note on reflector arrays" [Online]. Available. https://goo.gl/4cJkCF

\subsection{Epigraphs, Figures and Charts}

The epigraphs of the body of the article will be numbered in Arabic. They should go without a full box of capital letters, neither underlined nor bold. The numbering must be a maximum of three levels: 1. / 1.1. / 1.1.1. At the end of each numbered epigraph will be given an enter to continue with the corresponding paragraph.

The charts must be included in the text according to order of appearance, numbered in Arabic and subtitled with the description of the content, the subtitle should go at the top of the table justified to the left.

Figures can be linear drawings, maps or black and white halftone or color photographs in 300 dpi resolution. Do not combine photographs and line drawings in the same figure.

Design the figures so that they fit eventually to the final size of the journal $21 \mathrm{x}$ $28 \mathrm{~cm}$. Make sure inscriptions or details, as well as lines, are of appropriate size and thickness so that they are not illegible when they are reduced to their final size (numbers, letters and symbols must be reduced to at least $2.5 \mathrm{~mm}$ in height After the illustrations have been reduced to fit the printed page). Ideally, the linear illustrations should be prepared at about a quarter of their final publication size.

Different elements in the same figure should be spelled a, b, c, etc.
Photographs should be recorded with high contrast and high resolution. Remember that photographs frequently lose contrast in the printing process. Line drawings and maps should be prepared in black.

The text of the figures and maps must be written in easily legible letters.

If the figures have been previously used, it is the responsibility of the author to obtain the corresponding permission to avoid subsequent problems related to copyright.

Each figure must be submitted in a separate file, either as bitmap (.jpg, .bmp, .gif, or .png) or as vector graphics (.ps, .eps, .pdf).

\section{Submission process}

The manuscript must be sent through the OJS system of the journal, <https://goo. gl/JF7dWT $>$, the manuscript should be uploaded as an original file in .pdf without author data and anonymized according to the above; In complementary files the complete manuscript must be loaded in .doc or .docx (Word file), that is to say with the data of the author (s) and its institutional ascription; Also the numbered figures should be uploaded in independent files according to the corresponding in the manuscript (as bitmap .jpg, .bmp, .gif, or .png or as vector graphics .ps, .eps, .pdf). It is also obligatory to upload the cover letter and grant of rights as an additional file.

All authors must enter the required information on the OJS platform and only one of the authors will be responsible for correspondence.

Once the contribution has been sent the system will automatically send the author for correspondence a confirmation email of receipt of the contribution. 


\section{Editorial process}

Once the manuscript has been received in OJS, a first check by the editorial team of the following points:

- The topic is in accordance with the criteria of the journal.

- Must have the IMRDC structure.

- Must be in the INGENius format.

- Must use the IEEE citation format.

- All references should be cited in the text of the manuscript as well as charts, figures and equations.

- The manuscript is original; for this, software is used to determine plagiarism.

The assessment described above can take up to 4 weeks.

If any of the above is not complete or there is inconsistency, an email will be sent to the author to make the requested corrections.

The author will make the corrections and resend the contribution through an email in response to the notification and will also upload the corrected manuscript into OJS supplementary files.

The editorial team will verify that the requested corrections have been incorporated, if it complies, the manuscript will start the second part of the process that may be followed by the author through OJS, otherwise the author will be notified and the manuscript will be archived.

The second phase of the process consists of the evaluation under the methodology of double-blind review, which includes national and foreign experts considering the following steps:
- The editor assigns two or more reviewers for the article.

- After reviewing the article, the reviewers will submit the evaluation report with one of the following results.

- Publishable

- Publishable with suggested changes

- Publishable with mandatory changes

- Non publishable

- The editor once received the evaluation by the reviewers will analyze the results and determine if the article is accepted or denied.

- If the article is accepted, the author will be notified to make corrections if required and the corresponding editorial process will be continued.

- If the article is denied, the author will be notified and the manuscript will be archived.

- In the two previous cases the result of the evaluation of the reviewers and their respective recommendations will be sent.

The second phase of the process lasts at least 4 weeks, after which they will be notified to the author giving instructions to continue with the process.

\section{Publication}

The IngEnIUs Journal publishes two issues per year, on January 1st and July 1st, so it is important to consider the dates for sending the articles and their corresponding publication. Articles received until October will be considered for the January publication and those received until April for the July publication. 


\title{
UNIVERSIDAD POLITÉCNICA SALESIANA DEL ECUADOR
}

Javier Herrán Gómez, sdb

Rector

\author{
(C)Universidad Politécnica Salesiana \\ Turuhuayco 3-69 y Calle Vieja \\ Casilla postal 2074 \\ Cuenca, Ecuador \\ Teléfono: (+593 7) 2050000 \\ Fax: (+593 7) 4088958
}

Correo electrónico: srector@ups.edu.ec

\section{CANJE}

Se acepta canje con otras publicaciones periódicas.

Dirigirse a:

Secretaría Técnica de Comunicación

Universidad Politécnica Salesiana

Turuhuayco 3-69 y Calle Vieja

Casilla postal 2074

Cuenca, Ecuador

Teléfono: $(+5937) 2050000$ Ext. 1182

Fax: (+593 7) 4088958

Correo electrónico: rpublicas@ups.edu.ec

www.ups.edu.ec

Cuenca - Ecuador

Ingenius, Revista de Ciencia y Tecnología, publicación semestral, N.` 19, enero/junio 2018

John Calle Sigüencia, Editor Responsable revistaingenius@ups.edu.ec

Diseño de portada e impresión

Centro Gráfico Salesiano: Antonio Vega Muñoz 10-68 y General Torres.

Teléfono: (+593 7) 2831745

Cuenca - Ecuador

Correo electrónico: centrograficosalesiano@lns.com.ec 


\section{OTRAS PUBLICACIONES PERIÓDICAS DE LA UNIVERSIDAD}

UNIVERSITAS, Revista de Ciencias Sociales y Humanas.

LA GRANJA, Revista de Ciencias de la Vida.

ALTERIDAD, Revista de Educación.

RETOS, Revista de Ciencias de la Administración y Economía.

UTOPIA, Revista de Pastoral Juvenil Universitaria.

SOPHIA, Colección de Filosofía de la Educación.
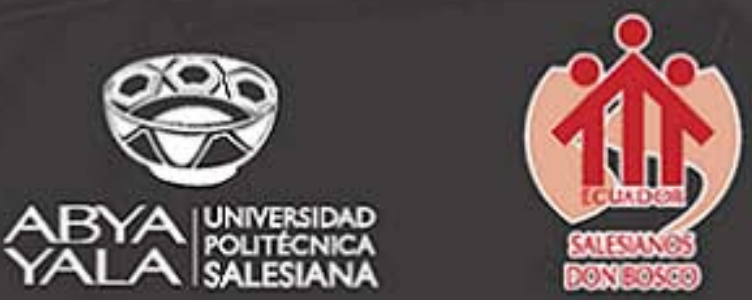